\title{
Lumped Element Kinetic Inductance Detectors
}

by

Simon Doyle

A thesis submitted to

Cardiff University

for the degree of

Doctor of Philosophy

April 2008 
UMI Number: U585117

All rights reserved

\section{INFORMATION TO ALL USERS}

The quality of this reproduction is dependent upon the quality of the copy submitted.

In the unlikely event that the author did not send a complete manuscript and there are missing pages, these will be noted. Also, if material had to be removed, a note will indicate the deletion.

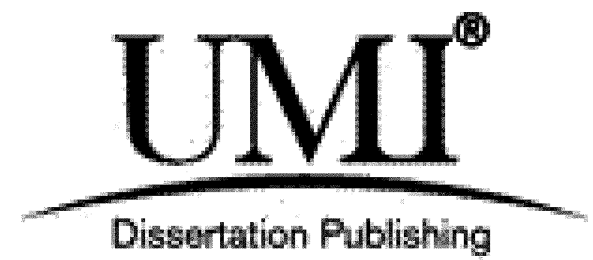

UMI U585117

Published by ProQuest LLC 2013. Copyright in the Dissertation held by the Author.

Microform Edition (c) ProQuest LLC.

All rights reserved. This work is protected against unauthorized copying under Title 17, United States Code.

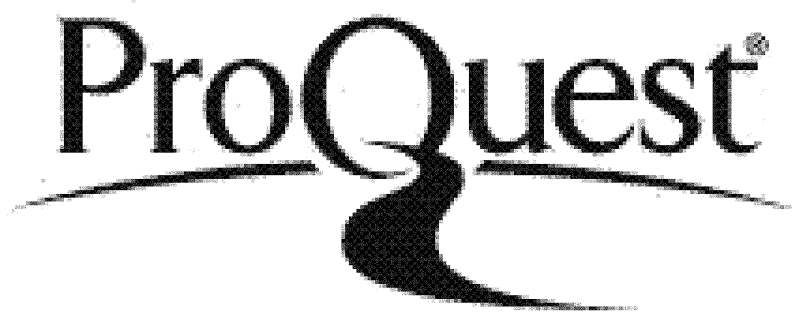

ProQuest LLC

789 East Eisenhower Parkway

P.O. Box 1346

Ann Arbor, MI 48106-1346 


\section{DECLARATION}

This work has not previously been accepted in substance for any degree and is not being concurrently submitted in candidature for any degree.

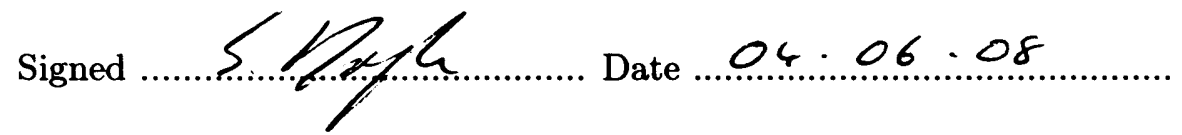

\section{STATEMENT 1}

This thesis is being submitted in partial fulfilment of the requirements for the degree of $\mathrm{PhD}$.

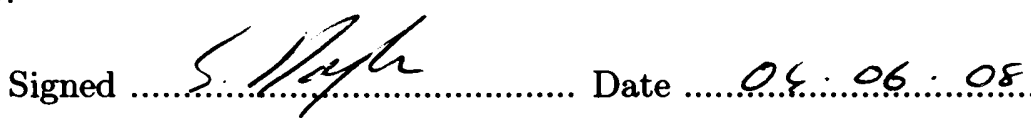

\section{STATEMENT 2}

The work presented in this thesis is all my own and carried out under the supervision of Prof. Phil Mauskopf and Prof. Adrian Porch with the following exceptions: (1) The data collected and presented in chapter 10 used a testbed designed and built by the Space Research Institute of the Netherlands (SRON). Analysis of this data was undertaken by myself with the advice of the team at SRON. (2) The fabrication of the devices tested in this thesis was performed by $\mathrm{Mr}$ Chris Duscombe at Cardiff University. (3) The plots in figure 7.13 to 7.17 were performed by myself and Dr Jin Zhang at Cardiff University.

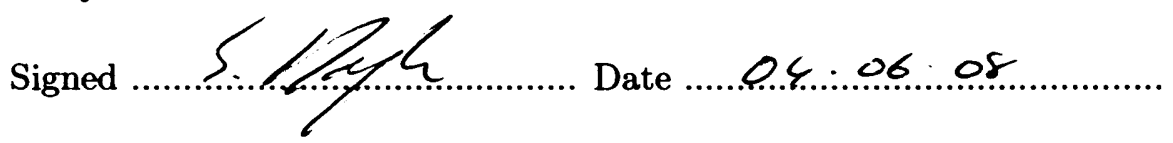

\section{STATEMENT 3}

I hereby give consent for my thesis, if accepted, to be available for photocopying and for inter-library loan, and for the title and summary to be made available to outside organisations.

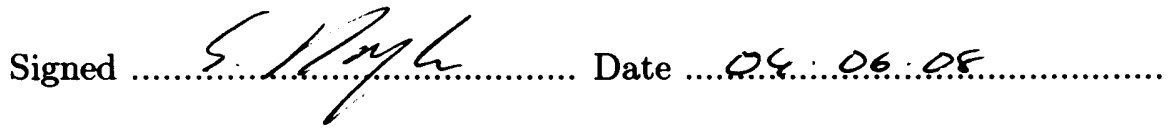

\section{STATEMENT 4}

I hereby give consent for my thesis, if accepted, to be available for photocopying and for inter-library loans after expiry of a bar on access previously approved by the Graduate Development Committee.

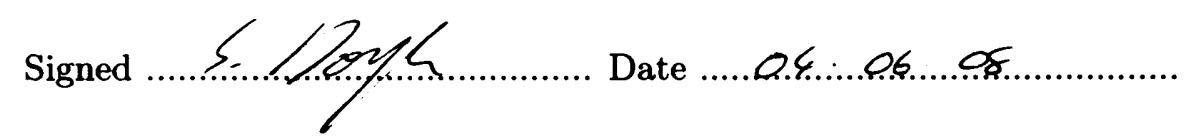




\section{ACKNOWLEDGMENTS}

The work presented throughout this thesis has been made possible with the support and guidance of many people who I wish to thank.

Firstly I would like to thank my supervisors Professor Phil Mauskopf and Professor Adrian Porch. Professor Mauskopf's excellent understanding over a vast range of Physics and Astronomy has helped to keep me motivated and provided me with an excellent project in which to be involved. His fantastic attitude to teaching and approach to supervision has not only helped me develop many skills but made the past four years thoroughly enjoyable. Professor Porch's supervision was crucial in understanding much of the superconductivity and Microwave theory. I am particularly grateful for the huge amounts of effort he made in helping me get to grips with some of the intricate details of this detector and showing great patience and understanding in the process. I wish also to thank Chris Dunscombe, who has not only provided excellent technical support for both clean-room and lab activities including the fabrication of all the Cardiff devises but also for being an fantastic office companion.

My friends have provided me with a much needed distraction from the lab at times to which I am eternally grateful. The many beers shared with Gustav Teleberg, Bruce Sibthorpe, James Cox, Richard Frewin and the numerous others who rounded off the week with a relaxing drink and topical debate will always be remembered fondly.

I would also like to thank my parents, Angela and Peader Doyle, who always encourage and supported my education and helped me achieve all that I have so far.

I owe a special thanks to my wonderful fiancée Alice. Her patience and understanding throughout the last four years has been second to none and despite suffering my absence due the commitments of this work has still always remained enthusiastic and inquisitive in understanding what it is I do. 


\section{ABSTRACT}

Kinetic Inductance Detectors (KIDs) provide a promising solution to the problem of producing large format arrays of ultra sensitive detectors for astronomy. Traditionally KIDs have been constructed from superconducting quarter-wavelength or halfwavelength resonator elements capacitivly coupled to a coplanar feed line. Photons are detected by measuring the change in quasi-particle density caused by the splitting of Cooper pairs in the superconducting resonant element. This change in quasi-particle density alters the kinetic inductance, and hence the resonant frequency of the resonant element. This arrangement requires the quasi-particles generated by photon absorption to be concentrated at positions of high current density in the resonator. This is usually achieved through antenna coupling or quasi-particle trapping. For these detectors to work at wavelengths shorter than around $500 \mu \mathrm{m}$ where antenna coupling can introduce a significant loss of efficiency, a direct absorption method needs to be considered. One solution to this problem is the Lumped Element KID (LEKID), which shows no current variation along its length and can be arranged into a photon absorbing area coupled to free space and therefore requiring no antennas or quasi-particle trapping. The work throughout this thesis studies the properties of the LEKID device though simulation and experimental data and lays the foundation for developing an optimised detector using this direct absorption approach. 


\section{Contents}

1 Introduction $\quad 1$

1.1 LEKID Basic concept . . . . . . . . . . . . . . . . . . 4

$\begin{array}{lll}2 & \text { Scientific motivation } & 7\end{array}$

$\begin{array}{lll}3 & \text { Superconductivity theory } & 13\end{array}$

3.1 The conductivity of normal metals . . . . . . . . . . . 14

3.2 Zero resistance and the Meissner effect . . . . . . . . . . . 15

3.3 The two fluid model $\ldots \ldots \ldots \ldots$

3.4 Internal inductance from the London model . . . . . . . . . . . . 21

3.5 The microscopic theory of superconductivity . . . . . . . . . . 26

3.6 The density of states and quasi-particle excitations . . . . . . . 31

3.7 Mattis-Bardeen theory . . . . . . . . . . . . . . . . 34

3.8 Impedance of a superconducting strip . . . . . . . . . . . . . 37

3.9 The quasi-particle equilibrium state . . . . . . . . . . . . . 42

4 Resonator theory and the KID concept 45

4.1 The parallel lumped resonator . . . . . . . . . . . . 45 
4.2 The series lumped resonator . . . . . . . . . . . . . 49

4.3 The quarter wave resonator $\ldots \ldots \ldots \ldots$. . . . . . . 51

4.4 Scattering parameters for resonance circuits . . . . . . . . . 55

4.5 Principles of operation of a KID . . . . . . . . . . . . 58

4.6 The lumped element KID . . . . . . . . . . . . . 60

4.7 Modelling the LEKID resonator . . . . . . . . . . . . . 66

5 LEKID Design $\quad 71$

5.1 Initial LEKID designs $\ldots \ldots \ldots$. . . . . . . . . . . . 71

5.2 Microwave coupling to the LEKID . . . . . . . . . . . . . 77

5.3 Sonnet simulations of the LEKID . . . . . . . . . . . 80

5.4 Chip design $\ldots \ldots \ldots \ldots \ldots$

6 Analytical LEKID simulations $\quad 87$

6.1 Response of the LEKID to change in temperature . . . . . . . . 87

6.2 LEKID response to change in quasi-particle density . . . . . . . . 91

6.3 Noise in KID devices . . . . . . . . . . . . . . . . 97

6.3.1 Detector bandwidth . . . . . . . . . . . . 97

6.3 .2 GR noise . . . . . . . . . . . . . 98

$\begin{array}{lll}7 & \text { Optical coupling to KID devices } & 101\end{array}$

7.1 Antenna coupled distributed KIDs . . . . . . . . . . . . 101

7.2 Quasi-particle trapping ................... 103

7.3 Partially filled distributed absorbers . . . . . . . . . . . . 106 
7.3.1 Impedance of a superconducting film at high frequency . . . 107

7.3.2 Modelling absorption . . . . . . . . . . . . 108

7.4 Inaccuracies in the absorption model $\ldots \ldots \ldots$

7.5 HFSS simulations and the solid sheet approximation $\ldots \ldots 117$

7.6 LEKID Hybrid solutions . . . . . . . . . . . . . . . . 120

7.7 Optical coupling conclusion . . . . . . . . . . . . 124

8 Cardiff experimental set up $\quad 127$

8.1 LEKID chip packaging . . . . . . . . . . . . . . 127

8.2 Low temperature system . . . . . . . . . . . . . . . 129

8.3 Cooler control and thermometry . . . . . . . . . . . . 131

8.4 Microwave readout electronics . . . . . . . . . . . . 135

$8.5 \mathrm{RF}$ components . . . . . . . . . . . . . . . 137

8.5.1 Variable step attenuator . . . . . . . . . . . 137

8.5 .2 RF Amplifiers . . . . . . . . . . . . . . . 138

8.5 .3 IQ Mixer . . . . . . . . . . . . . 138

8.6 Optical set up . . . . . . . . . . . . . 139

9 Experimental data $\quad 141$

9.1 First LEKID run . . . . . . . . . . . . . . . . . . 141

9.2 Second LEKID run . . . . . . . . . . . . . . . . . . . . . 144

9.3 Skewing of resonance curves $\ldots \ldots$. . . . . . . . . . 148

9.4 Back-bending . . . . . . . . . . . . . . . 151

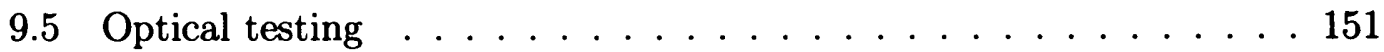


9.6 Predicted optical efficiency . . . . . . . . . . . . . . 158

9.7 Experimental data conclusion . . . . . . . . . . . . 159

10 Electrical NEP measurements $\quad 163$

10.1 SRON test set up . . . . . . . . . . . . . 165

10.2 Responsivity measurements . . . . . . . . . . . . 166

10.3 Quasi-particle lifetime measurements . . . . . . . . . . . 171

10.4 Phase and amplitude noise measurement $\ldots \ldots$. . . . . . . 172

10.5 NEP Calculation . . . . . . . . . . . . . . 176

10.6 Optical testing revisited . . . . . . . . . . . . 177

$\begin{array}{ll}11 \text { Conclusion } & 181\end{array}$

$\begin{array}{lr}\text { Appendices } & 187\end{array}$

$\begin{array}{lr}\text { A Derivation of equations } & 187\end{array}$

A.1 Perfect conductivity and complex impedance . . . . . . . . . . 187

A.2 The Meissner effect . . . . . . . . . . . . . . . 188

A.3 Kinetic and internal magnetic inductance . . . . . . . . . . . . . 190

A.4 Transmission line equations . . . . . . . . . . . . . . 195

Bibliography 


\section{List of Figures}

1.1 LEKID basic circuit diagram . . . . . . . . . . . . . . 4

1.2 Amplitude and phase response of a LEKID resonator $\ldots \ldots \ldots$

1.3 Multiplexed LEKID concept $\ldots \ldots \ldots \ldots \ldots \ldots$

2.1 Synthetic spectrum showing the SED of a typical galaxy undergoing modest rates of star formation . . . . . . . . . . . . . 9

3.1 The two fluid model $\ldots \ldots \ldots \ldots \ldots \ldots$

3.2 Current density approximations for thick and thin films . . . . . . 22

$3.3 \mathrm{Lm}$ and $\mathrm{Lk}$ vs film thickness . . . . . . . . . . . . . . . . 24

3.4 Total internal inductance vs $\lambda \ldots \ldots \ldots \ldots$

3.5 Variation in total internal inductance . . . . . . . . . . . 25

3.6 Formation of a Cooper pair . . . . . . . . . . . 27

3.7 Available momentum states for pair formation . . . . . . . 28

3.8 Temperature dependance of the energy gap . . . . . . . . . 30

3.9 The density of states for aluminium . . . . . . . . . . 32

3.10 Schematic of the density of states in a superconductor . . . . . . 33

$3.11 \sigma_{1} / \sigma_{n}$ vs $\mathrm{T}$ for aluminium at $7 \mathrm{GHz} \ldots \ldots 36$ 
$3.12 \sigma_{2} / \sigma_{n}$ vs $\mathrm{T}$ for aluminium at $7 \mathrm{GHz} \ldots \ldots . \ldots 37$

$3.13 \mathrm{R}$ vs $\mathrm{T}$ for aluminium strip . . . . . . . . . . . . 40

$3.14 \mathrm{Xs}$ vs $\mathrm{T}$ for aluminium strip . . . . . . . . . . . . 41

$3.15 N_{q p}$ vs T...................... 43

4.1 The Parallel Lumped Resonator. Here the supply has zero impedance 46

4.2 Loaded parallel resonator . . . . . . . . . . . . . . . 48

4.3 The series resonator being driven with a supply with zero impedance. 49

4.4 The series resonator being driven with a supply with impedance $Z_{\text {load }} .50$

4.5 Schematic of the Quarter Wave resonator . . . . . . . . . . 51

4.6 Current distribution in a typical coplanar line . . . . . . . . . 61

4.7 A schematic of A LEKID device . . . . . . . . . . . . . 62

4.8 The LEKID resonator simplified as a complex impedance $\left(Z_{\text {res }}\right)$. . 63

4.9 Typical Sonnet layout . . . . . . . . . . . . . . 68

5.1 Schematic of the parallel plate capacitor . . . . . . . . . . 72

5.2 Schematic of an 8 finger inter-digital capacitor. . . . . . . . . 73

5.3 Sonnet simulation layout for deducing capacitance . . . . . . . 75

5.4 Sonnet simulation for measuring LEKID inductance . . . . . . . . 76

5.5 Mutual inductance model based on a microstrip feedline . . . . 78

5.6 HFSS model of the vector B field . . . . . . . . . . . . 79

5.7 Bi-refringent dielectric constants of sapphire. . . . . . . . . . . . 80

5.8 Schematic of the standard LEKID design . . . . . . . . . . . . 81

5.9 Simulated current in a typical LEKID design. . . . . . . . . . . . . 82 
5.10 Plot of simulated mutual inductance vs coupling distance for a typical LEKID device. . . . . . . . . . . . . . . . . . . . 84

5.11 Plot of simulated S21 curves for various coupling distances. Here a 1600 square meander has been simulated with $5 \times 10^{-7} \Omega$ /square sheet resistance . . . . . . . . . . . . . . . . 85

5.12 Plot of simulated $Q_{L}$ vs coupling distance . . . . . . . . . 85

6.1 S21 Amplitude response of an aluminium LEKID from $250-300 \mathrm{mK} \quad 89$

6.2 S21 Phase response of an Aluminium LEKID from 250-300 mK . . 90

6.3 IQ response of an Aluminium LEKID from 250-300 $\mathrm{mK} \ldots . . . . .91$

6.4 LEKID S21 phase response . . . . . . . . . . . . . . . 93

6.5 LEKID S21 phase response variation with $\mathrm{g} \ldots \ldots$. . . . . . . 95

6.6 Variation in coupling coefficient with temperature . . . . . . . 96

$6.7 d T / d N_{q p}$ for LEKIDs of varying film volumes . . . . . . . . . . 96

6.8 LEKID GR noise . . . . . . . . . . . . . . . . . . . . 99

7.1 S21 Antenna Coupled Distributed KID . . . . . . . . . . . 102

7.2 Quasi-particle Trap Schematic . . . . . . . . . . . . . . . 104

7.3 Schematic of a mesh absorber . . . . . . . . . . 107

7.4 Complex conductivity as a function of frequency for an Aluminium film at $100 \mathrm{mK} \ldots \ldots \ldots \ldots$. . . . . . . . . . . . . . . . . . . .

7.5 Polarised response of a LEKID meander $\ldots \ldots$. . . . . . . . 109

7.6 Optical model for a LEKID illuminated directly . . . . . . . . . 110

7.7 Absorption for LEKID illuminated directly . . . . . . . . . . . 111

7.8 Optical model for a back illuminated LEKID . . . . . . . . . . . 113

7.9 Absorption for LEKID illuminated through the substrate $\ldots \ldots 113$ 
7.10 Optical model for a back illuminated LEKID with an AR coating . 115

7.11 Absorption for LEKID illuminated through a substrate with an AR

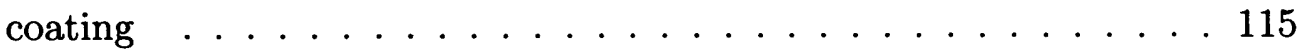

7.12 HFSS model of the solid sheet approximation. . . . . . . . . . 118

7.13 HFSS simulations of the solid sheet approximation. . . . . . . . 119

7.14 Hybrid LEKID absorber schematic . . . . . . . . . . . . 121

7.15 Hybrid LEKID absorption simulation . . . . . . . . . . . . 122

7.16 HFSS Simulation of the absorption for a single material meander . . 123

7.17 HFSS Simulation of the absorption for a single material meander . . 123

8.1 Chip Carrier . . . . . . . . . . . . . . . . . . . . 128

8.2 Sorption cooler schematic . . . . . . . . . . . 130

8.3 The test cryostat $\ldots \ldots \ldots . \ldots \ldots \ldots$

8.4 RF Chain schematic . . . . . . . . . . . . . 136

8.5 Warm electronics ... . . . . . . . . . . . 137

8.6 Optical chip carrier . . . . . . . . . . . . . . . . . . . 139

9.1 LEKID curve fitting example . . . . . . . . . . . . . . 143

9.2 LEKID Transmission vs Temperature . . . . . . . . . . . . . 145

9.3 Loaded and unloaded quality factors for LEKIDs 11 and $12 \ldots 146$

9.4 LEKID 11 Resonant trajectory . . . . . . . . . . . . . . 147

9.5 Photo of chip LEKID2 . . . . . . . . . . . . . . . . . 147

9.6 LEKID skewing examples . . . . . . . . . . . . . . . . 148

9.7 Parasitic model . . . . . . . . . . . . . . . . . . . . . 149 
9.8 Simulated LEKID skewing examples . . . . . . . . . . . . . . 150

$9.9200 \mu m$ bandpass filter transmission . . . . . . . . . . . 153

9.10 Raw I time stream $\ldots \ldots \ldots$. . . . . . . . . . . . . . . . . .

9.11 Raw Q time stream . . . . . . . . . . . . 157

9.12 Filtered I and $\mathrm{Q}$ time streams $\ldots \ldots \ldots$

9.13 Raw phase time stream . . . . . . . . . . . . . 159

9.14 Filtered phase time stream $\ldots \ldots \ldots$

9.15 Phase power spectrum $\ldots \ldots \ldots 161$

9.16 Predicted Optical Efficiency of LEKID $5 \ldots 162$

10.1 SRON cold RF chain . . . . . . . . . . . . . . . . 166

10.2 Measuring Phase in the IQ plane $\ldots \ldots 168$

10.3 Measured IQ circles for LEKID $5 \ldots 169$

10.4 Phase response of LEKID $5 \ldots \ldots$. . . . . . . . . . . . . . .

10.5 Quasi-particle lifetime measurements . . . . . . . . . . . 171

10.6 Phase noise of LEKID $5 \ldots \ldots$. . . . . . . . . . . . . . . . . .

10.7 Amplitude noise of LEKID $5 \ldots \ldots \ldots$

10.8 Phase noise of LEKID 5 low readout power . . . . . . . . . 175

10.9 Electrical NEP for LEKID $5(1-10000 \mathrm{~Hz}) \ldots \ldots \ldots$

10.10Electrical NEP for LEKID $5(1-100 \mathrm{~Hz}) \ldots \ldots \ldots$

10.11Schematic of stray light absorption in LEKID $5 \ldots 179$

11.1 Full LEKID Array Concept $\ldots$. . . . . . . . . . . . . 185 
A.1 Magnetic field decay in a superconductor . . . . . . . . . . . . 191

A.2 Superconducting strip cross-section . . . . . . . . . . . . . 191 


\section{List of Tables}

2.1 Future detector requirements . . . . . . . . . . . 10

$3.1 \tau_{0}$ values for various superconductors $\ldots \ldots \ldots 44$

5.1 Sonnet Simulation Summary . . . . . . . . . . . 76

5.2 Chip 3 design details $\ldots \ldots \ldots$. . . . . . . . . . 86

8.1 Cooler cycle procedure $\ldots \ldots \ldots 133$

9.1 Chip 5 Design values and measured $f_{0} \ldots \ldots 141$

9.2 LEKID run 2 summary . . . . . . . . . . . . . . . . 144

9.3 Radiated blackbody power . . . . . . . . . . . 155

9.4 Estimated Optical Noise Equivalent Powers . . . . . . . . . 156 


\section{Chapter 1}

\section{Introduction}

The amount of energy required to break a Cooper pair in a superconductor into two unbound electrons (quasi-particles) is twice the energy gap $(\Delta)$ and is of the order of $k_{B} T_{c}$ where $T_{c}$ is the superconducting transition temperature. Superconductors can therefore be used as detect any particle that has more than this amount of energy, i.e. photons with frequency greater than $\nu=k_{B} T_{c} / h$ or phonons generated by high energy particle interactions inside a crystal lattice. In order to make use of this potential, we need not only to be able to couple the signal that we want to detect into the superconductor, but also accurately measure the relative number of Cooper pairs and quasiparticles inside the superconductor. When used in this way, superconducting detectors are similar to semiconducting photoconductors or phonon sensors but with potentially much better sensitivity.

Superconductors are widely used in bolometric detectors as sensitive thermometers operating at the superconducting transition (transition edge superconducting - TES - bolometers). This is because below the superconducting transition the DC resistance of a superconductor is equal to zero and therefore a DC readout 
has no sensitivity to temperature changes or other pair breaking events inside the superconductor. TES bolometers are limited in sensitivity by the thermal isolation of the absorber and limited in the speed of response by the heat capacity of the absorber [1]. In contrast, the sensitivity of an ideal measurement of the relative number of Cooper pairs and quasi-particles to input power potentially improves exponentially as the temperature is reduced below the critical temperature as it is fundamentally limited by quasiparticle generation and recombination noise. This means that this detection technique offers potential advantages over TES detectors in sensitivity, dynamic range and speed of response.

The Microwave Kinetic Inductance Detector (MKID) concept was proposed as an $\mathrm{AC}$ method of measuring the relative fraction of paired and unpaired electrons in superconductors in the early part of this decade by Caltech and JPL $[2,3]$ and since then has rapidly grown to the point of being implemented in multi-frequency demonstrator instruments working in millimetre and submillimetre bands [4], the optical and near infrared and in the X-ray. The potential of the MKID has been realised by many instrumentation groups around the world as being a a strong candidate for the next generation of ultra-sensitive cryogenic detectors. The MKID has the very attractive features of having not only a very low theoretical noise limit but also being relatively easy to multiplex in the frequency domain.

The principal of operation for any KID device is to measure the change in quasi-particle population within the volume of a superconducting film upon photon absorption. Any photon with an energy $h f>2 \Delta$, if absorbed will break apart Cooper pairs resulting in an excess quasi-particle population $\left(n_{q p}\right)$. The result of this event is to alter the complex impedance of the film by increasing the kinetic inductance $\left(L_{k}\right)$. In practice the variance in $L_{k}$ with change in quasi-particle density is very small and requires the film to be fabricated in to a high quality factor 
( $Q$ factor) microwave resonance circuit to sense this variation. In this regime we can monitor the change in phase of a fixed tone microwave probe signal centred on the resonant frequency. The variation in $L_{k}$ upon photon absorption is now scaled by $Q$, which can be of order $10^{6}$ for a low loss superconducting resonator operating well below the superconducting transition temperature $\left(T_{c}\right)$ of the film [3]. The theoretical noise limit of these devices is governed by generation-recombination noise, which scales with temperature and film volume [5]. For a typical coplanar waveguide (CPW) aluminium MKID device operating at $100 \mathrm{mK}$ this noise is estimated to be around $10^{-20} \mathrm{~W} / \sqrt{\mathrm{Hz}}$.

The approach to date for creating high $\mathrm{Q}$ microwave resonators from superconducting films for the purpose of photon detection has been to fabricate distributed half-wave or quarter-wave resonators from CPW geometries. For these devices to act as photon detectors power must be coupled in to area of high current density using antenna structures or quasi-particle traps [3]. These methods have already been demonstrated to work for coupling to photons in the millimetre [4] and optical, UV and X-Ray [6], but to date have no viable solution for coupling to radiation in the mid infrared to far infrared (MIR-FIR 5-500 $\mu \mathrm{m}$ ) wavebands. This problem prompted the idea for a lumped element KID (LEKID) which shows no current variation along its length and also serves as a free space absorber. This new approach which has been developed by the detector group at Cardiff provides an elegant solution to the problem of coupling $\mathrm{THz}$ radiation to the sensitive element of a KID device, combining the properties of the absorbing area, detection and readout elements of the KID. 


\subsection{LEKID Basic concept}

In a resonant circuit fabricated from superconducting elements $(\mathrm{L}, \mathrm{C})$, varying the quasi particle density in the superconductor will alter the kinetic inductance $L_{k}$ which will in turn alter the resonant frequency $\omega_{0}$. When the value of $L_{k}$ is small compared to the total inductance of the resonant circuit $\left(L_{t o t}\right)$, the change in $\omega_{0}$ is proportional to $\alpha$ which is the ratio of kinetic inductance $L_{k}$ to total inductance $L_{t o t}\left(\alpha=L_{k} / L_{t o t}\right)$. The devices discussed in this thesis are created from an LRC series resonant circuit inductively coupled to a microstrip feedline. An equivalent circuit diagram of a lumped element KID is shown in figure 1.1

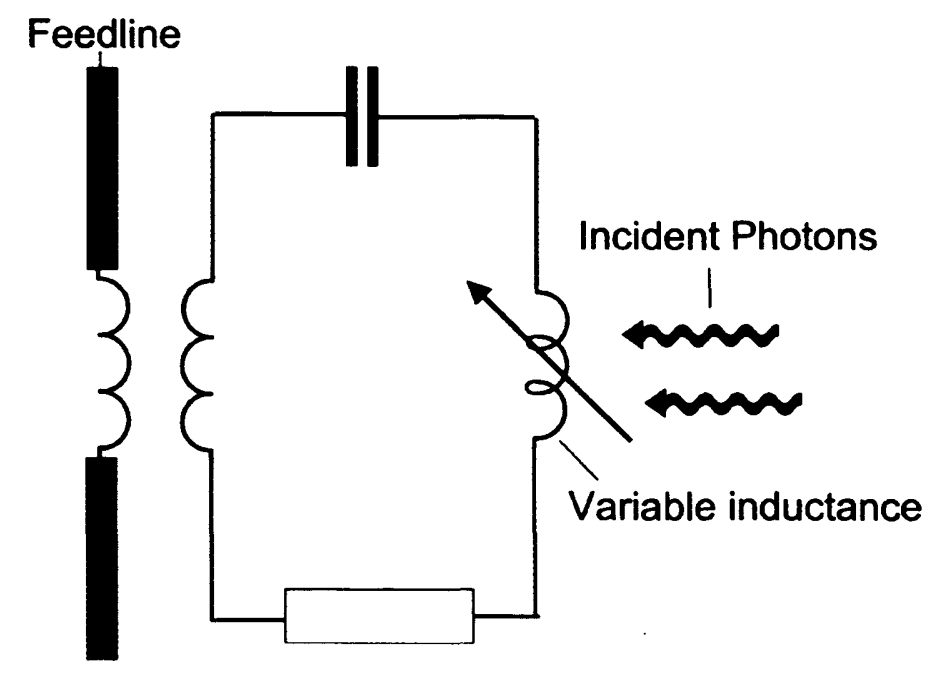

Figure 1.1: The LEKID basic concept. By creating a resonant circuit from superconducting components, the inductance can vary under photon absorption. The variation in inductance causes a shift in resonant frequency that can be sensed by the readout electronics, hence this circuit can be used as a photo-detector.

The presence of the resonator produces a change in amplitude and phase of a microwave probe signal transmitted along the microstrip feedline for frequencies close to $\omega_{0}$. This is shown by the solid curve in figure 1.2 .

A photon with energy $h f>2 \Delta$ can break Cooper pairs in the superconducting 
film making up the inductive section of the resonator altering $L_{k}$ and hence altering $\omega_{0}$. This change in $\omega_{0}$ is measured by observing the change in amplitude and phase of a fixed tone microwave probe signal of frequency $\omega_{0}$ transmitted past the resonator along the microwave feedline as the resonant feature shifts. This effect is demonstrated in 1.2 by the dashed curve.

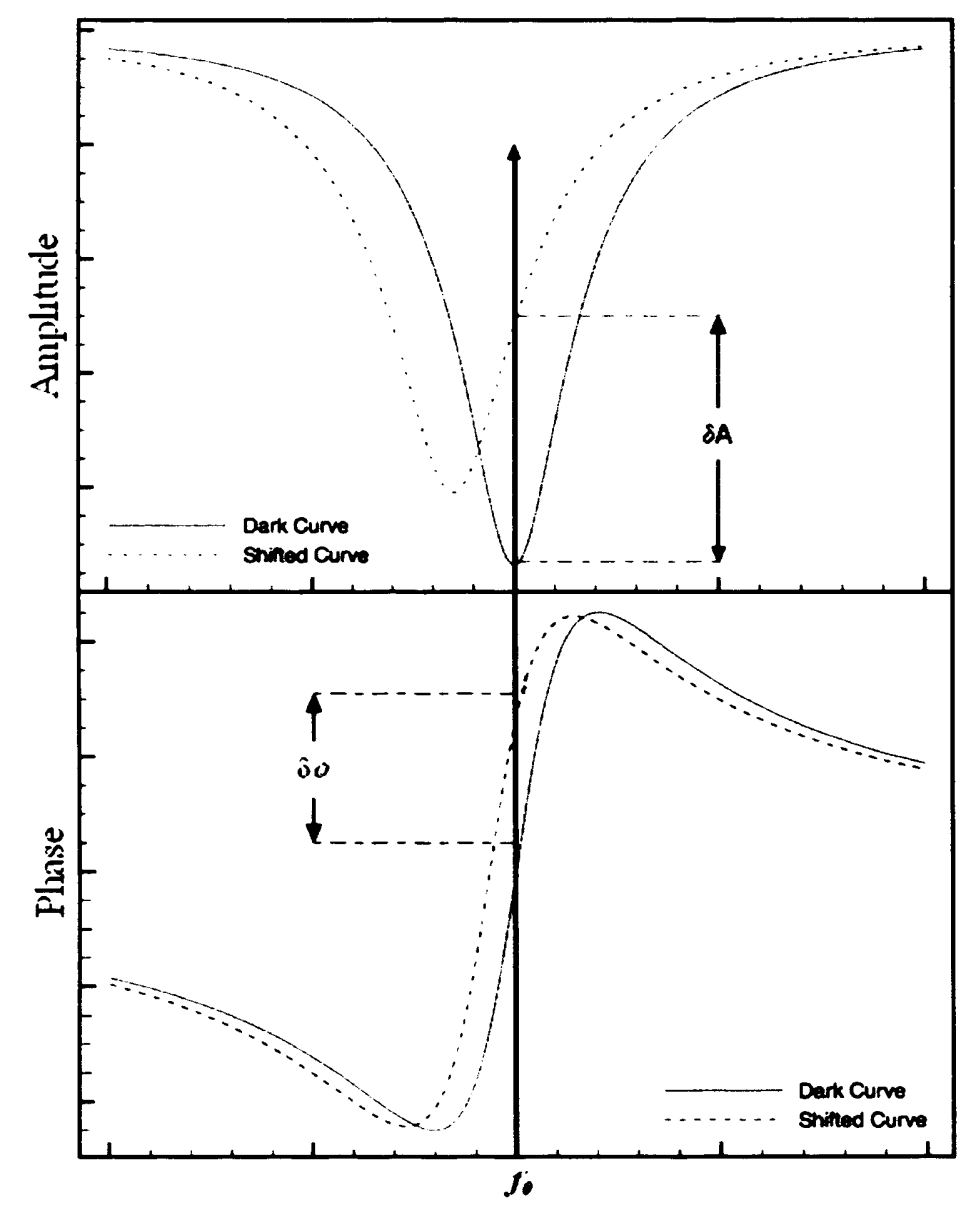

Figure 1.2: Amplitude and phase response of a LEKID resonator

The change in phase for a given change in frequency is determined by the slope of the phase curve $(d \phi / d \omega)$ which sets the responsivity of the detector. This response as we shall see later scales with the loaded quality factor of the resonator.

By fabricating many resonant elements of varying resonant frequency it is 
possible to multiplex many resonators onto a single feedline $[2,3]$. For the MKID this is achieved by varying the length of each quarter-wave resonator. The same principle applies to the LEKID but is achieved by varying the value of the capacitor in each resonator. This idea is demonstrated in figure 1.3.

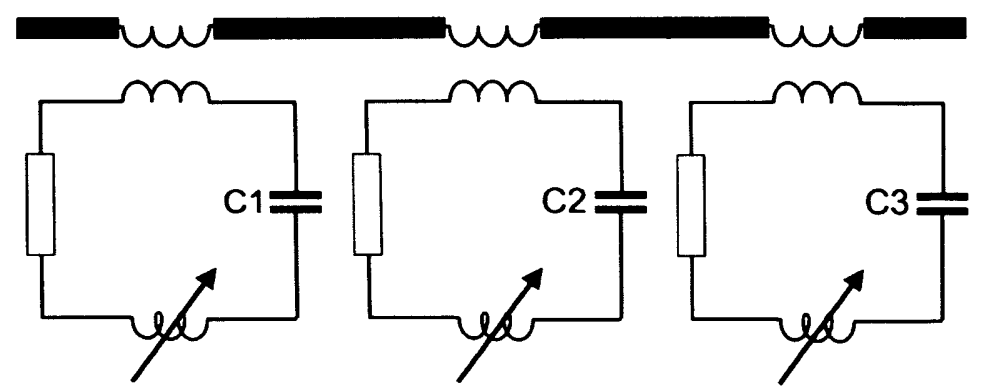

Figure 1.3: Multiplexed LEKID schematic. Here the resonant frequency of each resonant element is varied by varying the value of the capacitor. This makes it possible to multiplex many LEKID devices onto a single feedline

The relevant superconductivity and microwave theory for the operation of a KID device are quite complex and will be studied in detail throughout the following chapters. 


\section{Chapter 2}

\section{Scientific motivation}

One of the major gaps in observational astronomy and astrophysics exists from the mid infrared to far infrared (MIR-FIR 5-500 $\mu \mathrm{m}$ ) wavebands. Observations in the MIR-FIR range are especially useful in the study of star formation, Active Galactic Nuclei (AGN) and galaxy evolution. The importance of the MIR-FIR region can be best appreciated by looking at the Spectral Energy Distribution (SED) of a typical galaxy and noticing that half the energy is emitted in the MIR-FIR bands. This can be seen in figure 2.1. The main reason for this feature is absorption and re-emission of starlight by dust and gas in the interstellar medium as a thermal continuum and fine structure spectral lines. The photometric and spectroscopic analysis of the features seen in the MIR-FIR provides powerful tools for astronomers to constrain the various theories currently used to model star formation and galaxy evolution. Many important emission and absorption features are seen in the MIR-FIR and studies of these features will help determine redshifts and chemical composition of nearby and distant galaxies. Observations in the MIR-FIR also provide a method probing the cores of regions such as proto-stars and AGN which are enshrouded 
and optically obscured by dust.

The lack of advancement in this field compared to optical astronomy has been due to three factors. The first factor is background emission, owing to the fact that almost anything with moderate temperature $(T>10 \mathrm{~K})$ will radiate appreciably in the MIR-FIR bands. For ground based astronomy there is the added complication of atmospheric absorption. Water vapour in the atmosphere will absorb MIR-FIR radiation leaving only a few narrow atmospheric windows. One of these windows exists at $200 \mu m$ at some of the world's highest and driest sites (the Atacalma Desert, Chile and Antarctica). Ground based astronomy has been demonstrated in FIR bands at $350 \mu \mathrm{m}$ using the SHARC II instrument on the CSO [7] and at $200 \mu \mathrm{m}$ using Thumper on the JCMT [8]. Both these instruments were operating from telescopes situated on the summit of Mauna Kea, Hawaii. This site rarely provides the excellent weather conditions required for observing at these wavelengths. Suitable optical filters are also required to reject unwanted photon noise from outside the transmission band of the atmosphere.

Space based missions open up inaccessible regions of the MIR-FIR, and the planned launch of the Herschel Space Observatory [9] and James Webb Space Telescope (JWST) [10] will provide the most advanced observatories to date. Both these observatories will have detector arrays limited by the photon noise generated by the telescope background emission caused by the finite temperature of the dish ( $\approx 80 \mathrm{~K}$ for Herschel and $40 \mathrm{~K}$ for the JWST) . The third factor has been detector development. Silicon-based CCDs are insensitive to photon energies emitted in the MIR-FIR so new bolometric and photoconducting detectors have needed to be developed. These detectors need to be cooled to cryogenic temperatures $(T<4 K)$ and are difficult to fabricate in large arrays. However, research in this area and in cryogenics has made it possible to develop background limited arrays of moderate 
sizes for Herschel (139 pixel bolometer array for the $250 \mu \mathrm{m}$ band of SPIRE [11]) and up to mega-pixel sized arrays for MIRI (The Mid Infrared Instrument) on the JWST [12] using photoconductor devices.

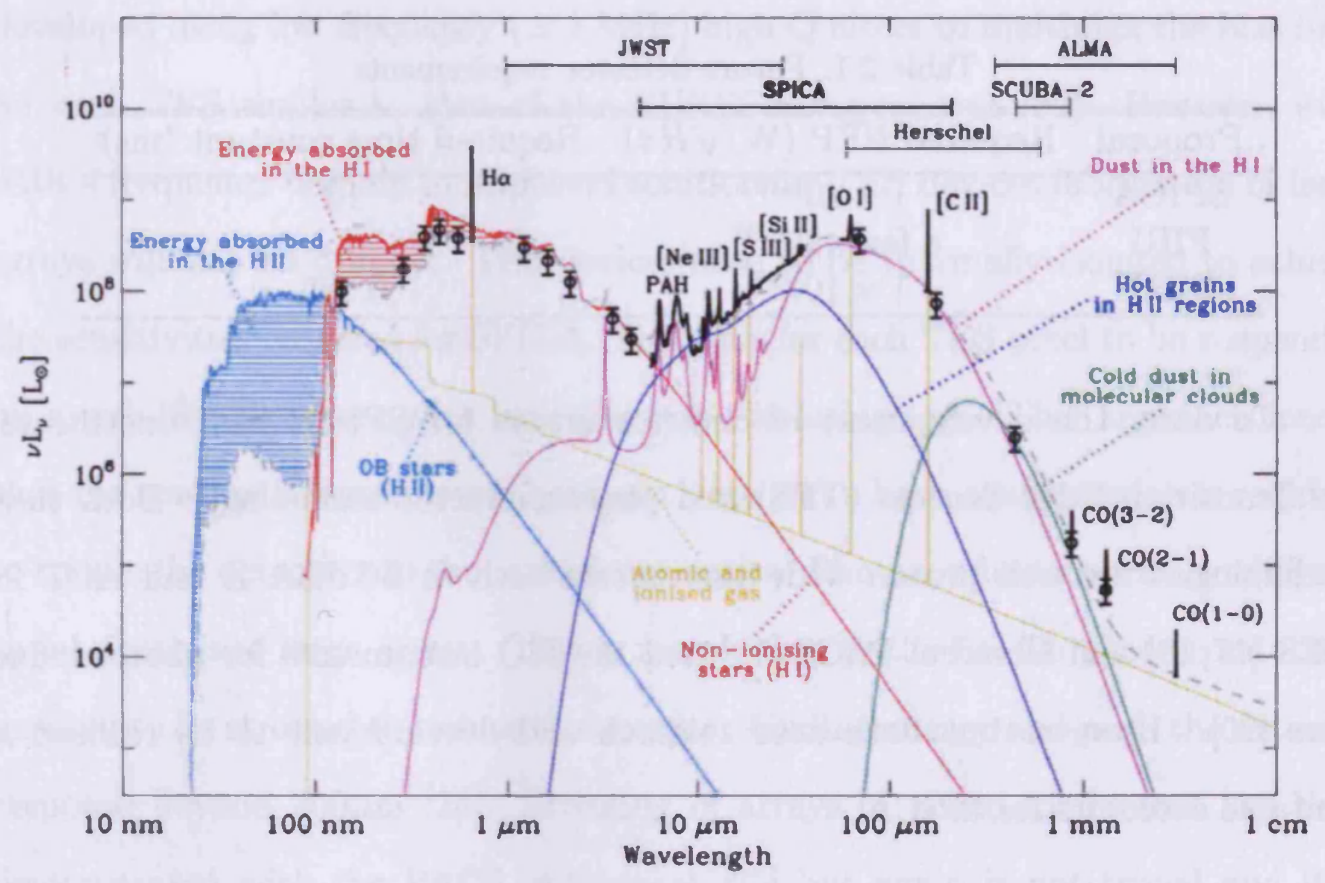

Figure 2.1: A synthetic spectrum showing the SED of a typical galaxy undergoing modest rates of star formation. This plot highlights many of the spectral features in the MIRFIR that could be observed using an observatory such as SPICA. [Source SPICA Cosmic Vision document (to be published)]

Although the advancement in the MIR-FIR bands will be considerably extended with the launch of Herschel and the JWST, observations of high redshift galaxies and faint nearby sources will be impossible due to the excess photon noise generated by the telescope. For this reason the future for MIR-FIR astronomy will be to develop space observatories with primary mirrors cooled to below $7 \mathrm{~K}$ with large fields of view capable of performing imaging spectroscopy and/or interferometry. Three proposed projects are the Japanese satellite SPICA $[13,14]$, the ESA project FIRI (a Far InfraRed Interferometer) [15] and the NASA project SPIRIT (Space Infrared Interferometric Telescope) [16]. These observatories will call for 
large arrays of detectors with sensitivities between one and two orders of magnitude better than current technologies to be background limited. Details of the requirements are shown in table 2.1

Table 2.1: Future detector requirements

\begin{tabular}{ccc}
\hline \hline Proposal & Required NEP $(\mathrm{W} / \sqrt{H z})$ & Required time constant $(\mathrm{ms})$ \\
\hline SPICA & $1 \times 10^{-19}$ & 10 \\
FIRI & a few $\times 10^{-20}$ & $?$ \\
SPIRIT & $1 \times 10^{-19}$ & 0.185 \\
\hline
\end{tabular}

To date, the development of detector arrays for SPICA has concentrated on Transition Edge Sensors (TES) and photoconductor technology. Both these technologies are well proven with instruments such as SCUBA II and ACT for TES $[17,18]$ and Herschel PACS [19] and the ISO instruments for photoconductors [20]. However but both need research and development to be realised as suitable detectors for SPICA.

Photoconductors have achieved sensitivities as low as a few $\times 10^{-18} \mathrm{~W} / \sqrt{\mathrm{Hz}}$ but can suffer from non linear effects producing multiple time constants [20]. Typical multiplexing regimes achieve around 8 detectors per channel. TES detectors have achieved sensitivities as low as a $4 \times 10^{-19} \mathrm{~W} / \sqrt{\mathrm{Hz}}$ [21] using isolating structures with sub-micron dimensions. Time Domain (TD) TES multiplexing ratios are at maximum on order of 32 pixels per channel (achieved for SCUBA II). Frequency Domain TES multiplexing ratios are at maximum on order of 100 pixels per channel (achieved for EURECA [22]). KIDs mux ratios are maximum on order of 1000 per channel.

Both of these detector technologies are difficult to fabricate and multiplex into large filled arrays as neither are naturally multiplex in the frequency domain as is the case with a KID device. Time domain multiplexing of TES devices in large 
arrays (over 10000 pixels) has been achieved for the SCUBA II instrument [23]; however this approach may not be practical for space-based applications. Solutions for multiplexing TES devices in the frequency domain for use in SPICA are being developed using low frequency ( $\simeq 1 \mathrm{MHz}$ ) high $\mathrm{Q}$ filters to multiplex the bias lines for each TES similar to that of the EURECA experiment [22]. However, even with a frequency domain multiplexed solution for TES devices fabrication of large arrays will still be difficult. TES devices need to be thermally isolated to achieve the sensitivities required for SPICA. This calls for each TES pixel to be suspended on a web-like structure with long thermally isolating legs. This approach means that the spacing between pixels becomes large and a horn configuration is required to couple the detectors to the instrument optics. Photoconductors are also difficult to fabricate into large arrays. This is mainly due to the Ga-Ge photo-conductors needing to be stressed to reduce the acceptor binding energy and push the detector response beyond $200 \mu \mathrm{m}$ [24]. Stressing of arrays of photo-conductors has been demonstrated with the PACS instrument [25] but again is not trivial and it is unlikely that this technology will achieve the required sensitivity specifications for SPICA. Although KIDs are a relatively new and unproven technology the rapid speed of development and advantages over more traditional technologies makes them a possible candidate detector technology for SPICA and other future FIR missions. 


\section{Chapter 3}

\section{Superconductivity theory}

In all elemental metals, a decrease in electrical resistance with reduced temperature is observed. However, in some metals such as niobium (Nb), aluminium ( $\mathrm{Al}$ ) and tin $(\mathrm{Sn})$ the DC resistance falls to zero below a certain temperature known as the critical temperature $\left(T_{c}\right)$. These materials are known as superconductors, the properties of which form the basis of many modern, ultra-sensitive cryogenic detectors including the Kinetic Inductance Detector (KID). Superconductors also display almost perfect diamagnetism, meaning that a superconductor will expel almost all magnetic field from within its bulk and only allow penetration of magnetic field within a small distance (typically $10-100 \mathrm{~nm}$ ) of the surface. This property is know as the Meissner effect. Superconductors fall into two basic categories, type I and II. Type I superconductors tend to be pure elements and will be discussed in the following chapter. Type II superconductors can range from metallic alloys through to ceramics. Type II superconductors tend to have higher transition temperatures and differ in their physical properties to Type I superconductors and will not be discussed in this report. 


\subsection{The conductivity of normal metals}

Unlike a superconductor, a normal metal does not have zero resistance at finite temperatures. The reason for this is that electrons scatter off the ions in the lattice of the metal. This causes a loss in the kinetic energy they have acquired from being accelerated by an applied electric field. The conductivity of a normal metal can be derived using the Drude model, here we will follow the working of Ashcroft and Mermin [26]. An electron gas is considered which will be accelerated by an applied field $\mathbf{E}$. The electrons respond to the field by being accelerated and gain momentum until they scatter off an ion and are given some new random direction and velocity. The scattering processes are characterized by an average time between scattering events $(\tau)$ and over many scattering events the electrons will have some average momentum in the direction of the field. This leads to equations 3.1 and 3.2 , which describe the conductivity of a normal metal using the Drude model.

$$
\sigma_{n}=\frac{\sigma_{0}}{(1-j \omega \tau)}
$$

Here $\sigma_{0}=n_{n} e^{2} \tau / \mathrm{m}$, where $n_{n}$ is the number density of electrons per unit vol$u_{m e}, e$ is the electron charge and $m$ is the effective electron mass. For aluminium at $77 \mathrm{~K}, \tau$ is typically around $6.5 \times 10^{-14} \mathrm{~s}$. Splitting 3.1 into its real and imaginary parts gives:

$$
\sigma_{n}=\frac{n_{n} e^{2} \tau}{m\left(1+\omega^{2} \tau^{2}\right)}-j \frac{n_{N} e^{2} \omega \tau^{2}}{m\left(1+\omega^{2} \tau^{2}\right)} .
$$

${ }^{*}$ The notation $n_{n}$ is used to denote normal electrons. As we shall see later in a superconductor we have two electron densities which need to be treated differently, hence the use of $n_{n}$ will become more apparent. 
This can be written as

$$
\sigma_{n}=\sigma_{1 n}-j \sigma_{2 n}
$$

The imaginary part of 3.2 arises from the fact that the electrons, due to their mass cannot respond instantaneously to the applied field. During the time $\tau$ the electrons under the influence of the applied field will gain momentum. If the field were reversed, the electrons would have to lose this momentum before moving in the new field direction. At microwave frequencies the period of the applied field is of the order $20 \mathrm{~ns}$, which is far larger than $\tau$ leaving the imaginary term in equation 3.3 typically 3 orders of magnitude less than the real term. For this reason the imaginary term is generally ignored in normal metals at microwave frequencies.

\subsection{Zero resistance and the Meissner effect}

Equations describing the electrodynamics of a superconductor were first put forward by F. London and H. London [27] in order to account for zero DC resistance and complete diamagnitism. The London model assumes that the electrons responsible for superconductivity do not scatter as they would in a normal metal. We will denote the superconducting (non-scattering) electron density as $n_{s}$ from here on. The derivation of the London equations is fairly lengthy and can be found in the appendix. Here we will give a brief description of their result.

An electron gas of density $n_{s}$ which does not scatter will accelerate in an electric field unhindered by the ions in the lattice. Under these conditions, applying a time varying field $\left(\mathbf{E}=\mathrm{E}_{0} \exp (\mathrm{j} \omega \mathrm{t})\right)$ leads to the first London equation: 


$$
\frac{d \mathbf{J}}{d t}=\frac{n_{s} e^{2}}{m} \mathbf{E}
$$

The conductivity of the electron gas from equation 3.4 is therefore given by:

$$
\sigma_{s}=-\mathrm{j} \frac{\mathrm{n}_{\mathrm{s}} \mathrm{e}^{2}}{\omega \mathrm{m}}
$$

It is worth noting that equation 3.5 can be obtained by letting $\tau=\infty$ in equation 3.2 .

Bulk superconductors display a property which cannot be described merely by perfect conductivity. This property is compete diamagnetism and is known as the Meissner effect. A superconducting sample will completely expel any magnetic flux density from within its interior by creating surface currents to cancel out any flux that penetrates into it. A magnetic field introduced ${ }^{\dagger}$ to a perfect conductor would be unable to penetrate into the bulk of the conductor due to similar screening currents generated on the surface. This property is demonstrated when looking at Maxwell's equation for Faraday's law of induction and Ampéres circuital law:

$$
\dot{\mathbf{B}}=-\operatorname{curl} \mathbf{E} \text {. }
$$

$$
\operatorname{curl} \mathbf{B}=\mu_{0}(\mathbf{J}+\dot{\mathbf{D}})
$$

Applying 3.6 and 3.7 to a perfect conductor shows that the conductor will

\footnotetext{
'By saying 'introduced' we mean that the sample was surrounded by zero field and an external magnetic field was then applied. Therefore the conductor sees a time varying field.
} 
work to prevent the field changing from within its bulk giving the result:

$$
\dot{\mathbf{B}}(\mathrm{x})=\dot{\mathbf{B}}(\mathrm{a}) \exp \left(\frac{-\mathrm{x}}{\sqrt{\mathrm{m} / \mu_{0} \mathrm{n}_{\mathrm{s}} \mathrm{e}^{2}}}\right)
$$

Here $x$ is the distance from the conductor surface and $\mathbf{B}(\mathrm{a})$ is the time varying magnetic flux density at the surface. We see that the penetration of the field rapidly decays as we move further from the surface of the conductor.

If a superconductor were simply a perfect conductor this would imply that if the sample was cooled below its $T_{c}$ within a magnetic field and then the field was removed, screening currents would be induced on the surface to sustain the field within the bulk. However, as mentioned earlier, a superconductor will completely expel any flux from within its bulk suggesting that it is not simply a perfect conductor. The solution proposed by London and London was to define a set of conditions through which not only $\dot{\mathbf{B}}$ but $\mathbf{B}$ decays as we move from the surface of the superconductor. This leads to equation 3.8 taking the form:

$$
\mathbf{B}(\mathrm{x})=\mathbf{B}(\mathrm{a}) \exp \left(\frac{-\mathrm{x}}{\sqrt{\mathrm{m} / \mu_{0} \mathrm{n}_{\mathrm{s}} \mathrm{e}^{2}}}\right)
$$

The field decays to $1 / e$ of its value at the surface within the distance $\lambda_{L}$ which is given by:

$$
\lambda_{L}=\sqrt{\frac{m}{\mu_{0} n_{s} e^{2}}}
$$

This distance is known as the London penetration depth. A derivation of 3.9 
can be found in the appendix.

Equation 3.9 describes the complete diamagnetism seen in superconductors and equation 3.4 describes the superconductor's resistanceless property. Collectively 3.4 and 3.9 are known as the London equations. It should made clear that the London equations, as originally derived, do not address any of the fundamental physics of superconductors but are in fact just a set of constitutive relations that describe the observed effects.

\subsection{The two fluid model}

As we shall see when considering the microscopic theory of a superconductor, when a superconductor passes through its transition temperature the population of electrons is divided in to two parts. One population consists of single electrons, which are known as quasi-particles and have a density $n_{q p}$. Quasi-particles are affected by scattering in the usual manner and exhibit loss. The other population consists of paired electrons with density $n_{s}$, which are bound together with an energy gap of $2 \Delta$. The paired electrons, known as Cooper pairs, are immune to scattering events and hence exhibit no loss. The effect of these two electron densities give rise to the two fluid model of conductivity for a superconductor, put forward by Gorter and Casimir in 1934 [28]. This model takes into account that the current in a superconducting material has two paths through which it can flow, one path through the superconducting electrons $\left(n_{s}\right)$ and one path through the normal electrons $\left(n_{q p}\right)$. The ratio of $n_{s} / n$ where $n$ is the total number of conducting electrons in the material is given by: 


$$
\begin{gathered}
n_{s} / n=1-\left(T / T_{c}\right)^{4} \\
\text { and } \\
n_{q p}=n-n_{s} .
\end{gathered}
$$

The conductivity of the two current paths can be calculated simply from 3.2 and 3.5. We shall denote the conductivity due to $n_{q p}$ as $\sigma_{n}$ and the conductivity due to $n_{s}$ as $\sigma_{s}$. At low frequencies the conductivity due to $\left(n_{s}\right)$ is far greater than that of the conductivity due to $n_{q p}$ and at DC we see the zero resistance effect superconductors are famous for. However, at higher frequencies, especially in the microwave region, $\sigma_{n}$ can play a considerable part in the conductivity. This is due to the kinetic inductance of the superconducting electrons. The inertia of these electrons produces a reactance giving us a large impedance at high frequencies. This effect is likened to an inductance as the energy drawn from the field $\mathbf{E}$ is stored in the kinetic energy of these non-scattering electrons. The effect also leads to the supercurrent $\mathbf{J}_{\mathbf{s}}$ lagging $\mathbf{E}$ by $90^{\circ}$. Looking at equation 3.5 , we can see that this reactance is inversely proportional to $n_{s}$. The quasi-particle resistance is inversely proportional to $n_{q p}$ so as we move from $T=0$ to $T_{c}$ we see that $\sigma_{s}$ decreases and $\sigma_{n}$ increases as the density $n_{s}$ reduces and the density $n_{q p}$ increases. The increase in $\sigma_{n}$ along with the decrease of $\sigma_{s}$ as we move to higher frequencies or temperature means that a larger fraction of current is shunted through the resistive path compared to lower frequency or temperature. The effect is that the superconducting material will exhibit higher losses as the frequency or temperature becomes higher. The two fluid model also neatly demonstrates the fact that at all temperatures below $T_{c}$ we observe zero DC losses. In this case the reactance of 
the superconducting electrons $\left(n_{s}\right)$ is zero and the finite impedance of the normal state electrons $\left(n_{q p}\right)$ is short circuited. Figure 3.1 gives a schematic representation of the two current paths in a superconductor.

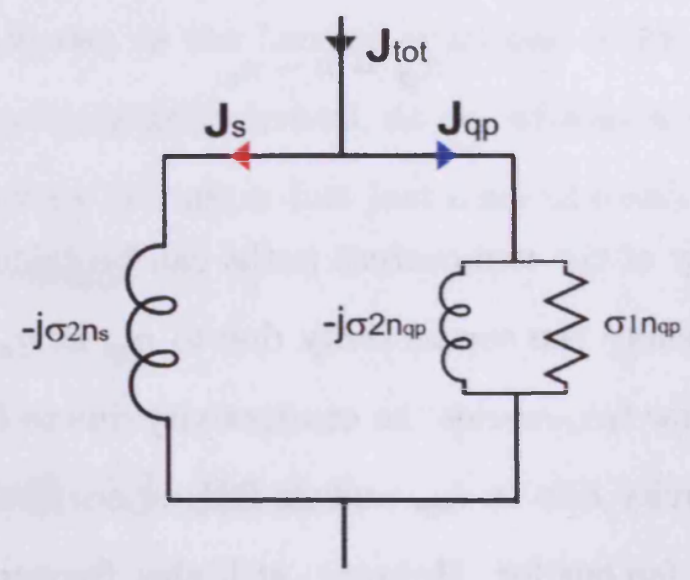

Figure 3.1: Two fluid model of a unit volume of superconductor. Here we see the superconducting current path denoted as $\sigma 2 n_{s}$ takes the form of an inductance with zero loss. The quasi-particle current path takes the form of a resistive path in parallel with an inductive path to account for the real and imaginary parts of the normal impedance.

As in equation 3.3 we can write the conductivity in terms of its real and imaginary parts. From 3.5 we see that the conductivity due to the superconducting electrons is purely imaginary. Neglecting the imaginary part of $\sigma_{n}$ we can write the conductivity as:

$$
\sigma=\sigma_{1}-j \sigma_{2}
$$

Here $\sigma_{1}$ is the conductivity associated with the quasi-particle density $\left(n_{q p}\right)$ and $\sigma_{2}$ is the conductivity associated with the superconductiing electron density $\left(n_{s}\right)$ 
The variation of the density $n_{s}$ with temperature means that the London penetration also varies with temperature. By writing $\sigma_{s}$ in terms of temperature from 3.11 we can write the temperature dependent London penetration depth as:

$$
\lambda_{L}(T)=\lambda_{L}(0)\left[1-\left(\frac{T}{T_{c}}\right)^{4}\right]^{\frac{-1}{2}}
$$

here $\lambda_{0}$ is the London penetration depth at zero kelvin.

\subsection{Internal inductance from the London model}

The total internal inductance of a superconducting strip comprises two parts, a kinetic inductance $\left(L_{k}\right)$ and a magnetic inductance $\left(L_{m}\right)$. The kinetic inductance is associated with the kinetic energy of the superconducting electrons $\left(n_{s}\right)$ and the magnetic inductance is associated with the magnetic field energy density created by $\mathbf{J}_{\mathbf{s}}$ stored within the superconducting volume. We can calculate the magnitude of both $L_{k}$ and $L_{m}$ by considering an isolated strip carrying a supercurrent $\mathbf{J}_{\mathbf{s}}$. Let us first look at the kinetic inductance $\left(L_{k}\right)$.

To calculate the kinetic inductance per unit volume we simply need to calculate the kinetic energy density of the electrons contributing to $\mathbf{J}_{\mathbf{s}}$. This is simply:

$$
K E=\frac{1}{2} n_{s} m v_{s}^{2}
$$

We can write the superconducting electron velocity $\left(v_{s}\right)$ in terms of current density $\left(J_{s}=-\mathrm{n}_{\mathrm{s}} \mathrm{e} v_{s}\right)$, leading to: 


$$
K E=\frac{1}{2} \frac{m}{n_{s} e^{2}} J_{s}^{2}=\frac{1}{2} \mu_{0} \lambda^{2} J_{s}{ }^{2}
$$

The general formula for $L_{k}$ is written as:

$$
U_{k}=\frac{1}{2} L_{k} I^{2}=\frac{1}{2} \mu_{0} \lambda^{2} \int_{s} J_{s}^{2} \mathrm{ds}
$$

where $U_{k}$ is the total kinetic energy per unit length. The surface integral (performed over the cross-section of the strip) takes into account the non-uniform current distribution. As mentioned before the current is confined to a small distance from the surface of size $\lambda$. For now we will consider the two cases shown in figure 3.2 which considers, (a) a film thickness $>>$ and (b) a film thickness $<<\lambda$. In both cases the width of the film (W) is assumed to be much grater than $\lambda$.

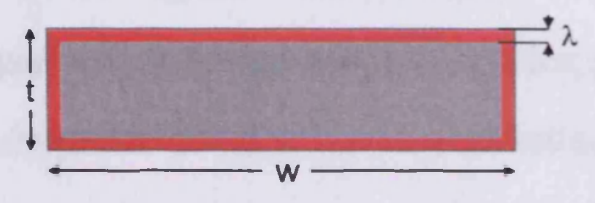

(a)

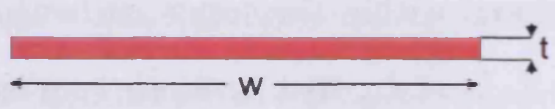

(b)

Current fiow No field penetration

Figure 3.2: Current density approximations for thick and thin films

For the case of $\mathrm{W}>t>>\lambda$ the effective cross-sectional area is $2 W \lambda$ (here we have ignored the small current density at the edges). In this case $J_{s}=\mathrm{I} / 2 \mathrm{~W} \lambda$ where, $\mathrm{I}$ is the total current in the strip. $L_{k}$ is now given by: 


$$
\frac{1}{2} L_{k} I^{2}=\frac{\mu_{0} I^{2}}{8 \mathrm{~W}^{2} \lambda^{2}} 2 w \lambda \rightarrow L_{k}=\frac{1}{2} \frac{\mu_{0} \lambda}{\mathrm{W}}
$$

In the case of $\mathrm{W}>>\lambda>>\mathrm{t}$ we can say that the current density in the film is uniform and given by $\mathbf{J}_{\mathbf{s}}=\mathrm{I} / \mathrm{Wt}$. Now the result for $L_{k}$ becomes:

$$
\frac{1}{2} L_{k} I^{2}=\frac{\mu_{0} I^{2} \lambda^{2}}{2 \mathrm{~W}^{2} t^{2}} \mathrm{~W} t \rightarrow L_{k}=\frac{\mu_{0} \lambda^{2}}{\mathrm{~W} t}
$$

Quite often we are working between the limits of $t<<\lambda$ and $t>>\lambda$. In this case we need to perform the surface integrals for current over the entire film cross-sectional area and take into account any variations in current density. The result (the derivation of which can be found in the appendix) is:

$$
\begin{aligned}
& L_{k}=\frac{\mu_{0} \lambda}{4 \mathrm{~W}}\left[\operatorname{coth}\left(\frac{t}{2 \lambda}\right)+\left(\frac{t}{2 \lambda}\right) \operatorname{cosec}^{2}\left(\frac{t}{2 \lambda}\right)\right] \\
& \quad \text { and } \\
& L_{m}=\frac{\mu_{0} \lambda}{4 \mathrm{~W}}\left[\operatorname{coth}\left(\frac{t}{2 \lambda}\right)-\left(\frac{t}{2 \lambda}\right) \operatorname{cosec}^{2}\left(\frac{t}{2 \lambda}\right)\right] .
\end{aligned}
$$

The results in 3.19 and 3.20 depend on film thickness as well as $\lambda$. This can be seen in figure 3.3. We can write an expression for the total internal inductance in $\mathrm{H} /$ square as:

$$
L_{\text {int }}=L_{m}+L_{k}=\frac{\mu_{0} \lambda}{2} \operatorname{coth}\left(\frac{t}{2 \lambda}\right)
$$




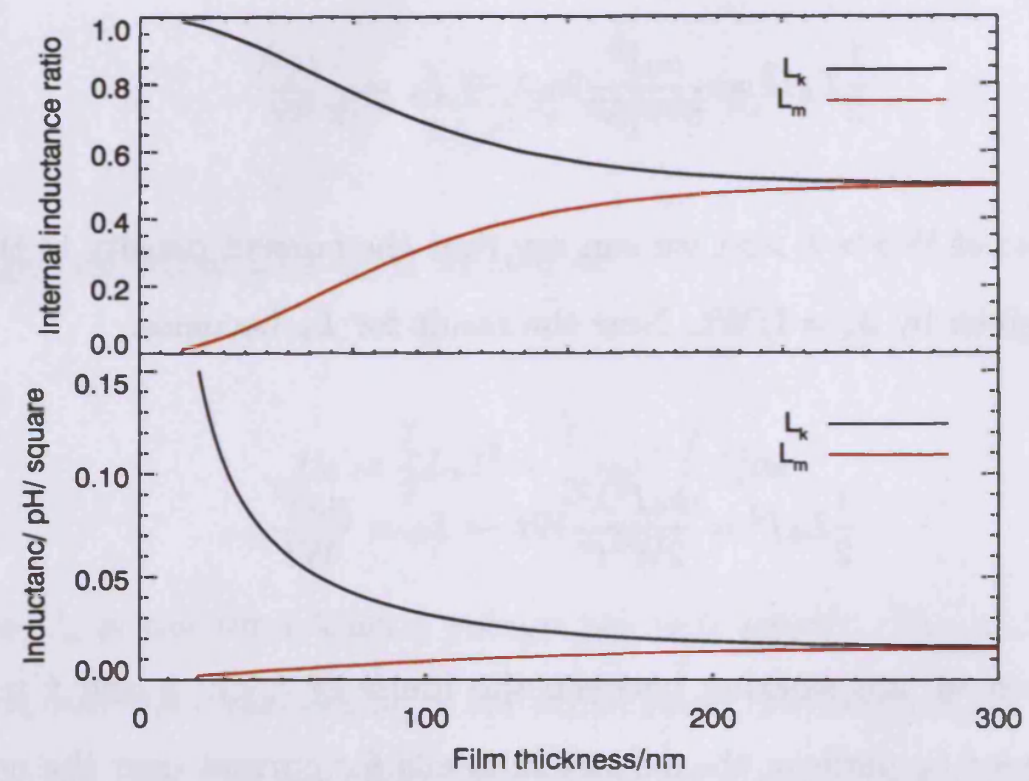

Figure 3.3: (Top) Ratios of $\mathrm{L}_{m}$ and $\mathrm{L}_{k}$ to total internal inductance. Note that in the case of a thick film $\mathrm{L}_{m}$ and $\mathrm{L}_{k}$ become equal. (Bottom) $\mathrm{L}_{k}$ and $\mathrm{L}_{m}$ in $\mathrm{pH}$ per square. This plot demonstrates a large increase in kinetic inductance as the film thickness is reduced. Both plots were created using a fixed $\lambda$ of $50 \mathrm{~nm}$.

As we shall see later the responsivity of a kinetic inductance detector is determined by the change in internal inductance of a film with a change in pair density and hence $\lambda$. Figures 3.4 and 3.5 show the variation of total internal inductance $\left(\mathrm{L}_{m}+\mathrm{L}_{k}\right)$ as a function of $\lambda$. The plots were generated using 3.19 and 3.20. 


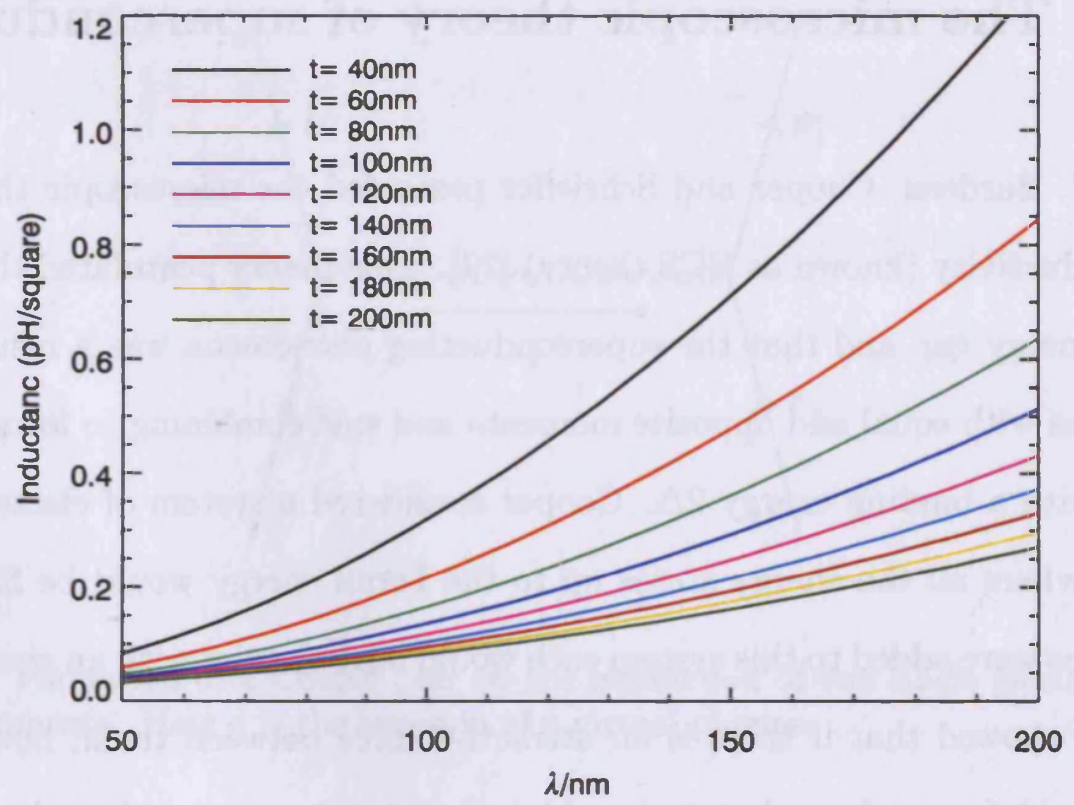

Figure 3.4: Total internal inductance for varying film thickness plotted in $\mathrm{pH} / \mathrm{square}$. Here we see a large increase in total internal inductance as we move to thinner films.

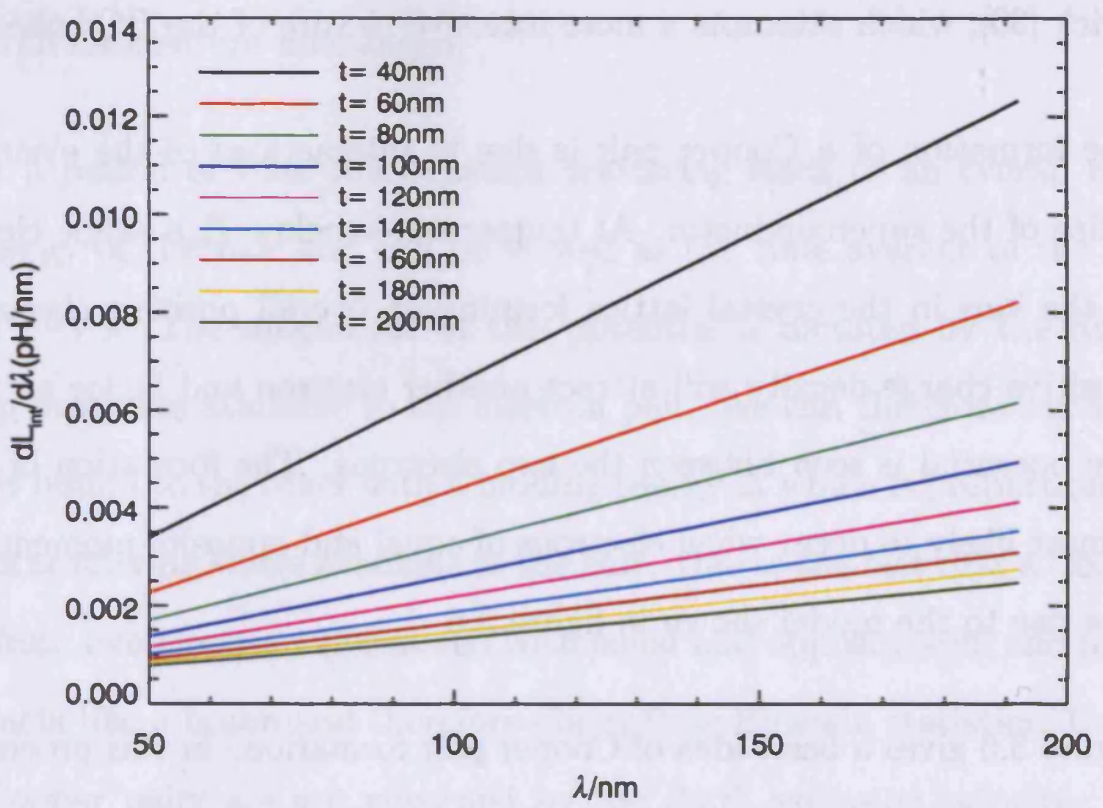

Figure 3.5: $d L_{i n t} / d \lambda$ for various film thickness. It is important to notice here that we see the largest change in total internal inductance with change in $\lambda$ for the thinnest films. 


\subsection{The microscopic theory of superconductivity}

In 1957, Bardeen, Cooper and Schrieffer presented the microscopic theory of superconductivity (known as BCS theory) [29]. This theory postulated the existence of an energy gap and that the superconducting phenomena was a result of single electrons with equal and opposite momenta and spin combining to form a "Cooper pair" with a binding energy $2 \Delta$. Cooper considered a system of electrons at zero kelvin where all the energy states up to the Fermi energy would be filled. If two electrons were added to this system each would have to exist with an energy $E>\epsilon_{f}$. Cooper showed that if there is an attractive force between them, however weak, they could form a bound state in which their total energy is less than $2 \epsilon_{f}$. The derivation of the BCS theory lies deep within the quantum mechanical theory of solid state physics. Here we will follow the explanation given by Rose-Innes and Rhoderick [30], which attempts a more intuitive picture of the BCS theory.

The formation of a Cooper pair is due to interactions of the electrons with the lattice of the superconductor. At temperatures below $T_{c}$ a single electron can distort the ions in the crystal lattice forming an overall positive charge density. This positive charge density will attract another electron and hence an attractive negative potential is seen between the two electrons. The formation of a Cooper pair is most likely to occur when electrons of equal and opposite momenta interact and give rise to the model shown in figure 3.6.

Figure 3.6 gives a basic idea of Cooper pair formation. In this process, overall momentum is conserved, that is, $\mathbf{P}_{\mathbf{1}}+\mathbf{P}_{\mathbf{2}}=\mathbf{P}_{\mathbf{1}}^{\prime}+\mathbf{P}_{\mathbf{2}}^{\prime}$. However this scattering takes place over a very short time and due to the Heisenberg uncertainty principle the energy of the final state can differ to the energy of the initial state by $\Delta E \geq$ $\hbar / \Delta t$. Such processes are called virtual processes and result in the emission of a 


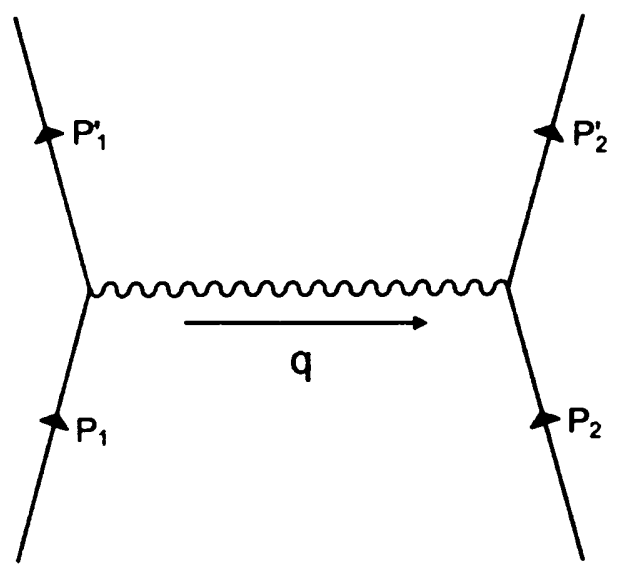

Figure 3.6: Formation of a Cooper pair by the interaction of two single electrons with opposite momenta. Here $q$ is the emission of a virtual phonon.

virtual phonon. Virtual processes can only exist if there is a second electron ready to absorb the virtual phonon emitted. The interaction between paired electrons can be interpreted as the two electrons constantly scattering off each other with their overall momentum unchanged.

Over a period of time this constant scattering leads to an overall reduction in the energy of the pair and can be viewed as the time average of the negative potential $(-V)$. The magnitude of this potential is dictated by the number of scattering processes available to the electron pair. We can therefore say that each electron is bound to the other with a binding energy $\Delta$ which is proportional to the number of scattering states available to the pair. Due to the fact that a Cooper pair is made from two electrons (fermions) with equal and opposite spin and momenta, the pair acts like a boson and therefore obeys Bose-Einstein statistics. Under this regime Cooper pairs are not governed by the Pauli exclusion principle and can therefore have more than one pair existing in the same quantum state, meaning that at zero kelvin all available electrons form Cooper pairs and condense into what is know as the superconducting ground state. 
The number of single electrons which can form pairs in a system is limited by the reduction in momentum states available. For example, for two electrons with momenta $\left(\mathbf{P}_{\mathbf{i}} \uparrow-\mathbf{P}_{\mathbf{i}} \downarrow\right)$ to scatter into the state $\left(\mathbf{P}_{\mathbf{j}} \uparrow \mathbf{P}_{\mathbf{j}} \downarrow\right)$ the state $\left(\mathbf{P}_{\mathbf{j}} \uparrow \mathbf{P}_{\mathbf{j}} \downarrow\right)$ must be empty. Taking a metal at zero kelvin, we can take a pair of electrons near the Fermi surface and allow them to scatter into new momentum states which allow them to form a Cooper pair as in figure 3.6, where the new momentum states must be empty. All the momentum states below the Fermi momentum are filled and from quantum mechanical arguments, the scattering process of figure 3.6 is only appreciable if the energy difference between the initial and final states is small. This limits the available momentum states to a spherical shell of thickness $\Delta \mathbf{P}=\mathrm{m} h \nu_{\mathrm{q}}$, where $m$ is the electron mass, $h$ is the Planck constant and $\nu_{q}$ is the average phonon frequency, which is of order half the Debye frequency. A schematic of the available states is shown in figure 3.7 .

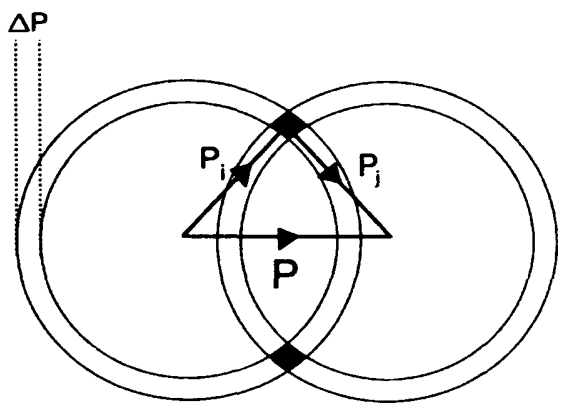

(a)

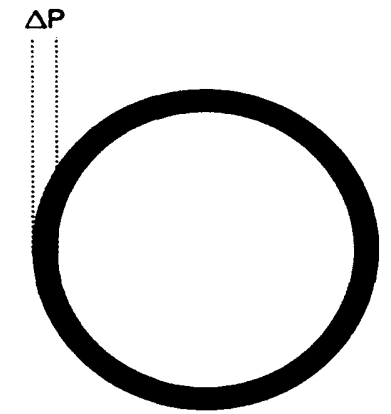

(b)

Figure 3.7: (a) Available momentum scattering states which conserves overall momentum (P) between two electrons with differing momentum. (b) Available momentum states which conserve momentum for two electrons with equal and opposite momenta $(\mathbf{P}=0)$. Note that in case (b) the maximum number of states is available and hence results in the greatest lowering of energy of the pair.

Figure 3.7 clearly shows that there is a limit to the total number of states available. This implies that as more and more pairs make use of these available 
states to form Cooper pairs, the reduction in energy is reduced due to the limited number of scattering states now left available to each pair. As more and more pairs form, a condition is reached where the reduction in energy of a pair can no longer outweigh the amount of energy they have which is in excess of $2 \epsilon_{F}$ and hence no more pairs can form.

The formation of Cooper pairs gives rise to an energy gap of size $2 \Delta$ either side of the Fermi energy. The size of this gap is temperature dependent due to the presence of quasi-particles. Imagine taking the situation at zero kelvin where the maximum number of pairs is formed and the gap is at a maximum. If we were now to break a pair by adding an energy greater than $2 \Delta$ through thermal agitation the electrons must now exist with an energy above the gap energy. The pair will now have individual momenta and their complementary states are now left empty. The implication of this is that the remaining pairs have fewer scattering states available as a quasi-particle with momentum $(\mathbf{P} \uparrow)$ without a partner $(-\mathbf{P} \downarrow)$ prevents the state $(\mathbf{P} \uparrow-\mathbf{P} \downarrow)$ being available to the remaining Cooper pairs. This reduction in available momentum states causes a reduction in the gap energy. As the temperature increases towards $T_{c}$, more quasi-particles are created reducing the number of available momentum states, hence reducing the gap until it becomes zero at $T_{c}$ where no pairs are formed.

As stated before, the gap energy is dependent on temperature but it is also dependent on the properties of the superconductor. The available momentum states are dictated by the Debye frequency of the material as well as the density of states at the Fermi surface. The gap energy is also dependant on the negative potential produced in a single scattering process $(V)$. The temperature dependance of the gap energy can be described by [31]: 


$$
\frac{1}{N(0) V}=\int_{-\hbar \omega_{D}}^{\hbar \omega_{D}} \frac{\tanh \left\{\left(\epsilon^{2}+\Delta^{2}(T)\right)^{\frac{1}{2}} / 2 k_{B} T\right\}}{2\left[\epsilon^{2}+\Delta^{2}(T)\right]^{\frac{1}{2}}} d \epsilon
$$

The value of $1 / N(0) V$ is material dependent and can be found from setting $\Delta$ to zero at the transition temperature, hence:

$$
\frac{1}{N(0) V}=\int_{0}^{\hbar \omega_{D}} \frac{\tanh \left\{\epsilon / 2 k_{B} T_{c}\right\}}{\epsilon} d \epsilon
$$

Figure 3.8 shows a plot of $\Delta$ vs $\mathrm{T}$ for aluminium. Here $\omega_{D}$ has been calculated from the Debye temperature $\left(\Theta_{D}\right)$ for aluminium which is $420 \mathrm{~K}$ [31]:

$$
\omega_{D}=\frac{\Theta_{D} K_{b}}{\hbar}
$$

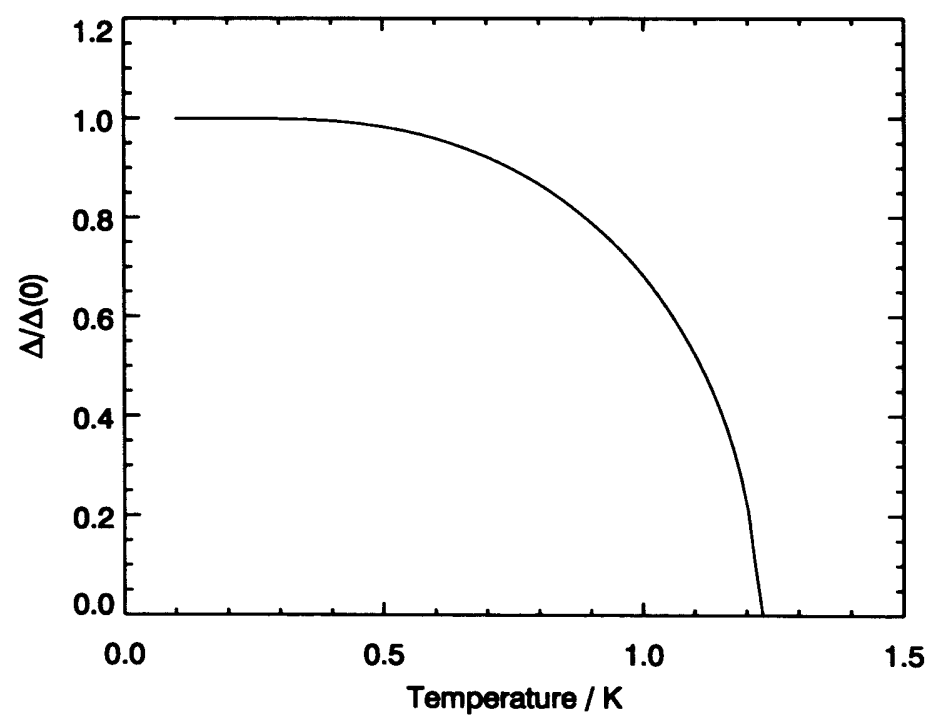

Figure 3.8: Temperature dependence of the energy gap for aluminium. Here $\Theta_{D}=420 \mathrm{~K}$ and $T_{c}=1.75$ were used in 3.23 to make the plot. Note that the plot does show some variation for $T_{c}$ (where $\Delta=0$ ). 


\subsection{THE DENSITY OF STATES AND QUASI-PARTICLE EXCITATIONS 31}

The plot in figure 3.8 shows that there is little change in the gap energy from its value at $0 \mathrm{~K}$ until around $T_{c} / 2$. The gap energy falls to zero at $T_{c}$. There are slight variances to the accepted values for $T_{c}$ and $\Delta(0)$ from measured aluminium films. These are caused by variations in the Debye temperature and the fact that equation 3.23 assumes a sharp cut-off at $\hbar \omega_{D}[32]$.

\subsection{The density of states and quasi-particle ex- citations}

The creation of the energy gap forces the density of states for $\epsilon_{F} \pm \Delta$ to be zero. The states which were present before the gap was introduced to the system are forced into regions either side of $\epsilon_{F} \pm \Delta$, forming a modified density of states that leaves the total number of states unchanged. The density of states is therefore temperature dependent as $\Delta$ is temperature dependent. The density of states relative to the Fermi energy is given by $[31,32]$ :

$$
N_{s}=\frac{N(0) E}{\left(E^{2}-\Delta^{2}(T)\right)^{\frac{1}{2}}}
$$

Here $N_{s}$ is the density of states in the superconducting state. $N_{n}(0)$ is the normal single spin density of electron states at the Fermi surface for the metal. The actual density of states above the gap energy is a function of energy; however, as we are only concerned with energies not too far from the $\epsilon_{F}$, we can take the density of states to be at a constant value of approximately $N_{n}(0)$. Figure 3.9 shows the density of states relative to $\epsilon_{f}$ for aluminium at $0 \mathrm{~K}$ and $1.1 \mathrm{~K}$. 


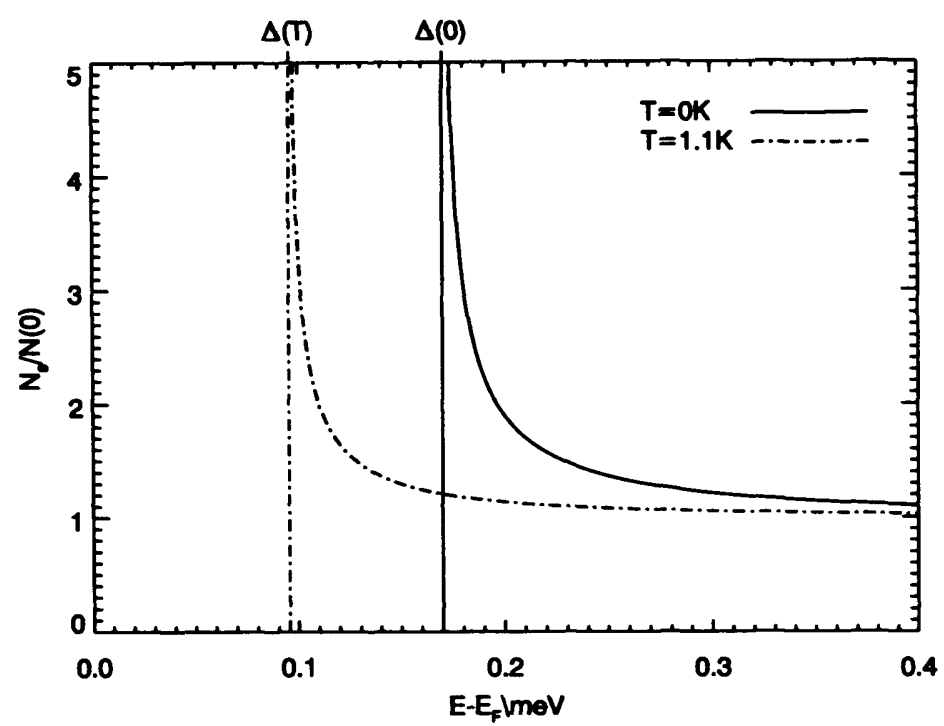

Figure 3.9: The density of states for both quasi-hole and quasi-particle excitations for aluminium at $0 \mathrm{~K}$ and $1.1 \mathrm{~K}$

Figure 3.9 shows that there are no energy states available between $\epsilon_{F}$ and $\epsilon_{F}+\Delta$. This leads to an enhanced density of states for $E>\epsilon_{F}+\Delta$ to keep the total number of states constant. Far beyond $E=\epsilon_{F}+\Delta$ the density of states returns to its usual value of $N_{n}(0)$. The density of states is symmetrical about $\epsilon_{F}$ with the states below $\epsilon_{F}$ being referred to as hole states. The schematic in figure 3.10 gives the basic concept of quasi-hole and quasi-particle excitations.

Figure 3.10 demonstrates the creation of quasi-particles and quasi-holes. As pointed out in the plot, one can find the number of quasi-particle excitations by integrating under the curve $F(E) N_{s}(E)$ from $\epsilon_{F}+\Delta \rightarrow \infty$. This operation essentially counts the number of states available and multiplies this by the probability that they are occupied (the Fermi-function). The number of quasi-particles created by adding energy to the system is equal to twice the number of pairs broken. However the reduction in the gap energy produces quasi-holes. This now allows some of the electrons below $\epsilon_{F}-\Delta$ to change their momentum and hence take part 


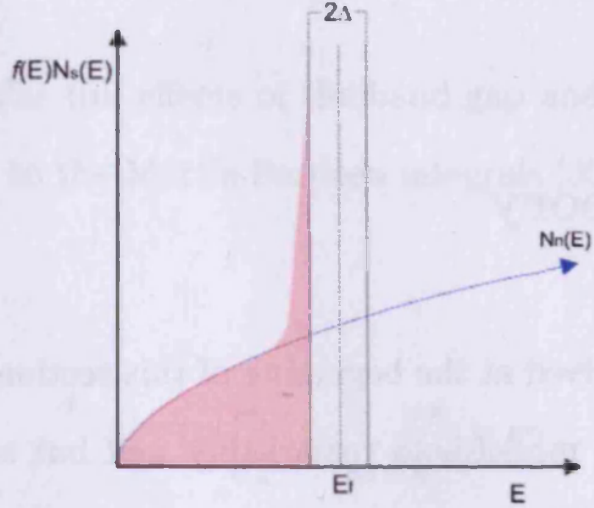

(a)

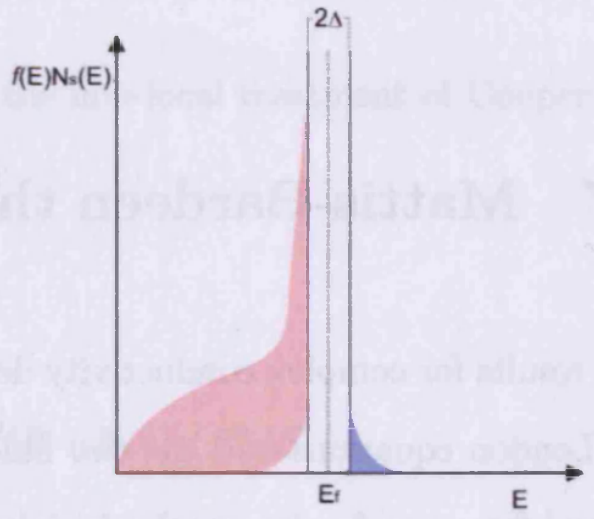

(b)

Figure 3.10: (a) A superconductor in the ground state. Here there are no quasi-particle excitations above $\epsilon_{F}+\Delta$. The states below $\epsilon_{F}-\Delta$ are completely filled hence the electrons here cannot change momentum and participate in conduction. All the electrons within the range $\epsilon_{F} \pm \Delta$ are paired and have energy $E=\epsilon_{F}$. (b) A superconductor in an excited state (non-zero temperature). Here the gap has reduced and created quasi-holes below $\epsilon_{F}-\Delta$ (empty space) and quasi-particles above $\epsilon_{F}+\Delta$ (blue). The quasi-particles are as a result of pair breaking and the number of quasi-particles formed can be found by integrating from $\epsilon_{F}+\Delta \rightarrow \infty$. Also plotted in (a) is the normal density of states $N_{n}$ for reference.

in normal state conduction $\left(\sigma_{1}\right)$. If we want to calculate the number of unpaired electrons which can take part in normal state conduction we need to perform the calculation in equation 3.26 [33].

$$
N_{q p}(T)=4 \int_{\epsilon_{F}+\Delta}^{\infty}\left(\frac{1}{1+\exp \left(E / k_{B} T\right)}\right)\left(\frac{N_{n}(0) E}{\left(E^{2}-\Delta^{2}(T)\right)^{\frac{1}{2}}}\right) d E
$$

The factor of 4 before the integral in equation 3.26 is due to the fact that each state can accommodate two electrons (spin up and spin down) and the density of states is for both quasi-holes and quasi-particles (i.e. the hole states allow electrons 
below $\epsilon_{F}-\Delta$ to contribute to normal state conductivity).

\subsection{Mattis-Bardeen theory}

The results for complex conductivity derived at the beginning of this section using the London equations and the two fluid model hold remarkably well but are not derived from any fundamental principles of superconductivity and do not take into account the idea of a band gap. Another assumption of the London model is that the electrons in the superconducting state are just simply electrons which do not scatter and will all be accelerated independently if an electric field is applied. Due to the finite size of a Cooper pair this is not the case. The interaction between electrons forming a Cooper pair happens over an average distance which is material dependent. This distance is know as the coherence length $(\xi)$. The coherence length from the microscopic BCS theory is given by [29]:

$$
\xi_{0}=\frac{0.18 \hbar \nu_{f}}{k_{B} T_{c}}
$$

Here $\nu_{F}$ is the Fermi velocity. The coherence length for a typical superconductor is of order $1 \mu \mathrm{m}$ and for aluminium is $1600 \mu \mathrm{m}$ [31]. Under these circumstances it is clear that we cannot describe the force on an electron pair simply by the local electric field. The non-local treatment of the London equations was suggested by Pippard [34], using similarities in the non-local theory for Ohm's law in rapidly varying spacial $\mathbf{E}$ fields and the second London equation. Similar situations are seen in normal conductors at high frequencies where the electron mean free path becomes comparable to the classical skin depth $\delta$. This is known as the anomalous 
skin effect, observed in pure metals at low temperature.

The full effects of the band gap and the non-local treatment of Cooper pairs leads to the Mattis-Bardeen integrals [35]:

$$
\begin{gathered}
\frac{\sigma_{1}}{\sigma_{n}}=\frac{2}{\hbar \omega} \int_{\Delta}^{\infty}[f(E)-f(E+\hbar \omega)] g(E) d E \\
+\frac{1}{\hbar \omega} \int_{\Delta-\hbar \omega}^{-\Delta}[1-f(E+\hbar \omega)] g(E) d E \\
\frac{\sigma_{2}}{\sigma_{n}}=\frac{1}{\hbar \omega} \int_{\Delta-\hbar \omega,-\Delta}^{\Delta} \frac{[1-2 f(E+\hbar \omega)]\left[E^{2}+\Delta^{2}+\hbar \omega E\right]}{\left[\Delta^{2}-E^{2}\right]^{\frac{1}{2}}\left[(E+\hbar \omega)^{2}-\Delta^{2}\right]^{\frac{1}{2}}} d E
\end{gathered}
$$

where $f(\eta)$ is the Fermi function

$$
f(\eta)=\frac{1}{1+\exp \left(\eta / k_{B} T\right)}
$$

and $g(E)$ is given by:

$$
g(E)=\frac{E^{2}+\Delta^{2}+\hbar \omega E}{\left(E^{2}-\Delta^{2}\right)^{\frac{1}{2}}\left[(E+\hbar \omega)^{2}-\Delta^{2}\right]^{\frac{1}{2}}}
$$

The Mattis-Bardeen integrals assume the extreme anomalous limit where $\lambda<<$ $\xi_{0}$

In the limit $k_{B} T<<\Delta(0)$ and $\hbar \omega<<\Delta(0)$ the Mattis-Bardeen integrals can 
be approximated by $[3,36]$ :

$$
\begin{gathered}
\frac{\sigma_{1}}{\sigma_{n}}=\frac{2 \Delta(T)}{\hbar \omega} \exp \left(-\Delta(0) / k_{B} T\right) K_{0}\left(\hbar \omega / 2 k_{B} T\right)\left[2 \sinh \left(\hbar \omega / 2 k_{B} T\right)\right] . \\
\frac{\sigma_{2}}{\sigma_{n}}=\frac{\pi \Delta(T)}{\hbar \omega}\left[1-2 \exp \left(-\Delta(0) / k_{B} T\right) \exp \left(-\hbar \omega / 2 k_{B} T\right) I_{0}\left(\hbar \omega / 2 k_{B} T\right)\right] .
\end{gathered}
$$

Here $I_{0}$ and $K_{0}$ are modified Bessel functions of the first and second kind respectively. Plots of $\sigma_{1} / \sigma_{n}$ and $\sigma_{2} / \sigma_{n}$ are shown in figures 3.11 and 3.12 .

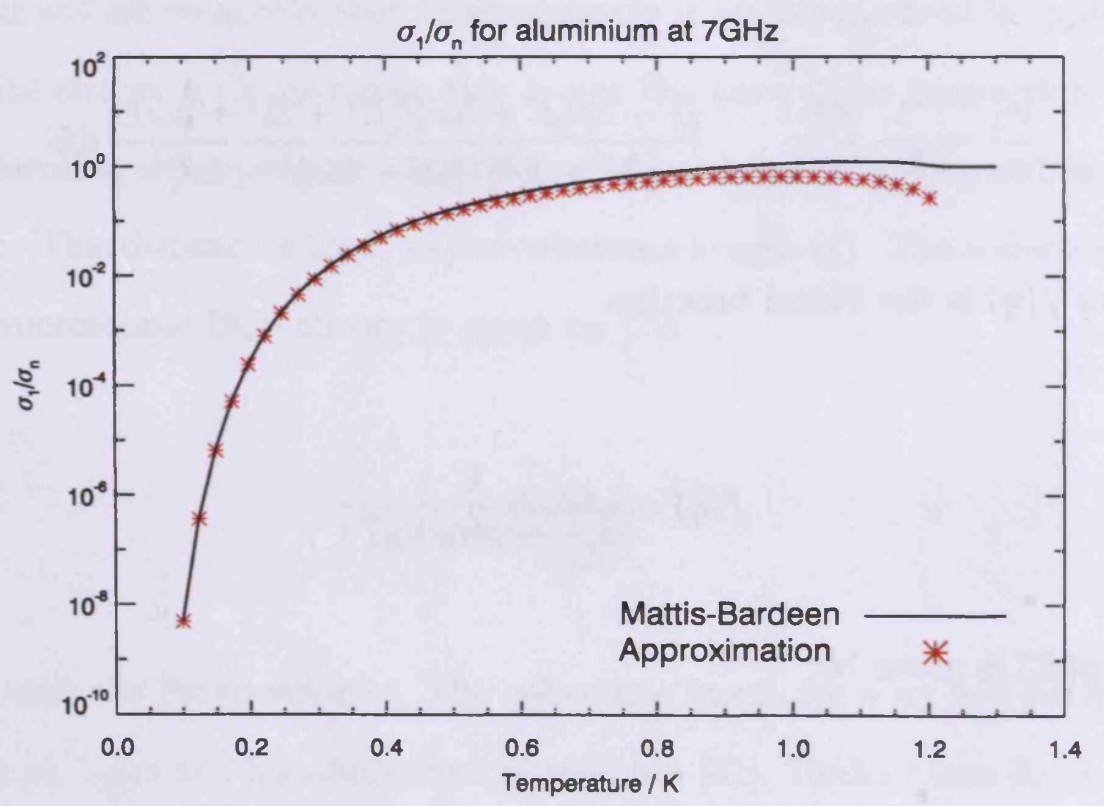

Figure 3.11: $\sigma_{1} / \sigma_{n}$ for aluminium at $7 \mathrm{GHz}$. This plot was created using the MattisBardeen integrals. Over-plotted is the approximation of $\sigma_{1} / \sigma_{n}$ for $\hbar \omega$ and $k_{B} T<<\Delta(0)$. We see good agreement from the approximation up until $500 \mathrm{mK}\left(T \approx T_{c} / 2.5\right)$

The overall effect of the Mattis-Bardeen integrals is a reduced conductivity leading to an increased penetration depth $\lambda$. The increase in $\lambda$ can be as much as an order of magnitude greater than that of the London penetration depth $\left(\lambda_{L}\right)$. 


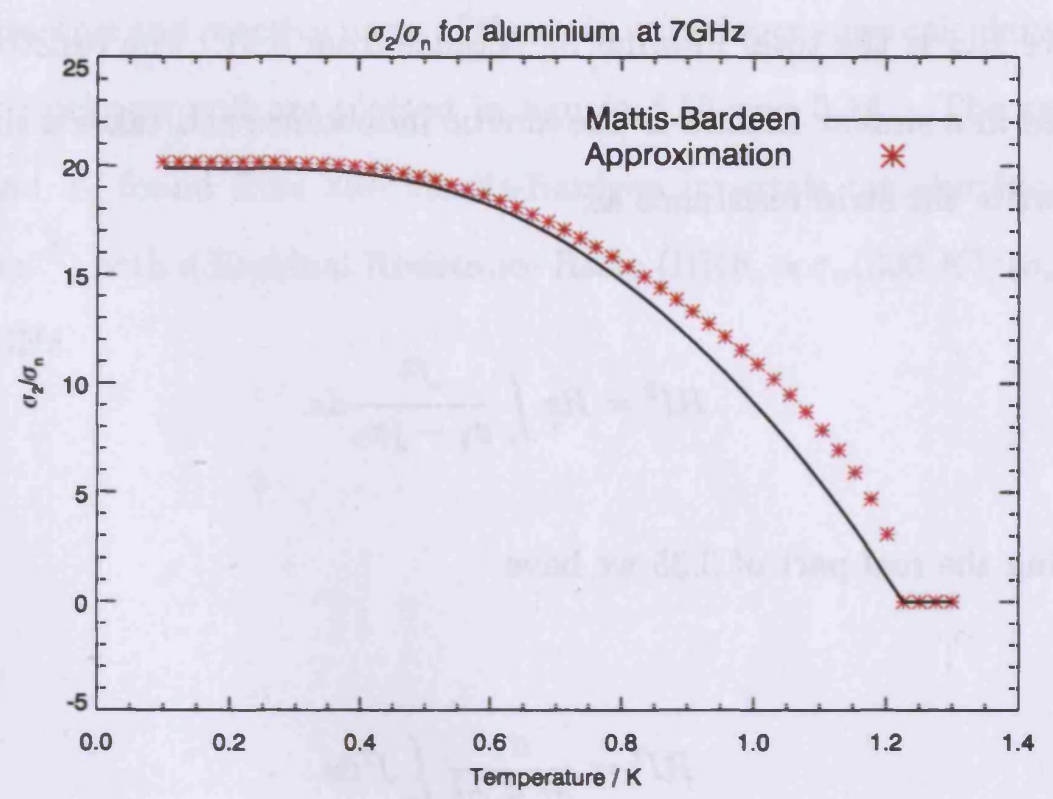

Figure 3.12: $\sigma_{2} / \sigma_{n}$ for aluminium at $7 \mathrm{GHz}$. Again this plot was created using the MattisBardeen integrals. Over-plotted is the approximation of $\sigma_{2} / \sigma_{n}$ for $\hbar \omega$ and $k_{B} T<<\Delta(0)$. Again we see good agreement from the approximation up until $500 \mathrm{mK}\left(T \approx T_{c} / 2.5\right)$.

This effect creates a significant difference in impedance calculations for clean films of materials with long coherence lengths such as aluminium.

\subsection{Impedance of a superconducting strip}

The impedance of a superconducting strip is split into two parts - a complex part which contains the kinetic and magnetic inductance $\left(L_{k}\right.$ and $\left.L_{m}\right)$ and a real part which is resistive. The complex part is found simply from the total internal reactance:

$$
X_{s}=\omega L_{t o t} \text {, }
$$


where $L_{t o t}$ is the total internal inductance from 3.21. The resistive part can be derived in a similar fashion as the kinetic inductance and takes a similar form. We can write the strip resistance as:

$$
R I^{2}=R e \int_{s} \frac{J^{2}}{\sigma_{1}-j \sigma_{2}} d s
$$

Taking the real part of 3.35 we have

$$
R I^{2}=\frac{\sigma_{1}}{\sigma_{1}^{2}+\sigma_{2}^{2}} \int_{s} J^{2} \mathrm{ds}
$$

The surface integral of $J^{2}$ can be written in terms of the kinetic inductance derived earlier giving:

$$
R=\frac{\sigma_{1}}{\sigma_{1}^{2}+\sigma_{2}^{2}} \frac{L_{k}}{\mu_{0} \lambda^{2}}=L_{k} \omega \frac{\sigma_{1} \sigma_{2}}{\sigma_{1}^{2}+\sigma_{2}^{2}}
$$

In the limit of $\sigma_{1}<<\sigma_{2}$ we can write $R$ as:

$$
R=L_{k} \omega \frac{\sigma_{1}}{\sigma_{2}}
$$

Writing both the resistive and reactive parts of the impedance in terms of conductivity then gives us a final expression for the strip impedance $Z_{s}$ :

$$
Z_{s}=R_{s}+j X_{s}=L_{k} \omega \frac{\sigma_{1} \sigma_{2}}{\sigma_{1}^{2}+\sigma_{2}^{2}}+j \sqrt{\frac{\mu_{0} \omega}{4 \sigma_{2}}} \operatorname{coth}\left(\frac{t}{2} \sqrt{\sigma_{2} \omega \mu_{0}}\right)
$$


The resistive and reactive parts of the strip impedance were calculated for films of varying thickness and are plotted in figures 3.13 and 3.14 . The calculations used $\sigma_{1}$ and $\sigma_{2}$ found from the Mattis-Bardeen integrals for aluminium $\left(\sigma_{n}=\right.$ $\left.3.7 \times 10^{7} \mathrm{Sm}^{-1}\right)$ with a Residual Resistance Ratio $\left(\operatorname{RRR}=\sigma_{n}(300 K) /\left(\sigma_{n}(4.2 K)\right)\right)$ of 2 at $7 \mathrm{GHz}$. 

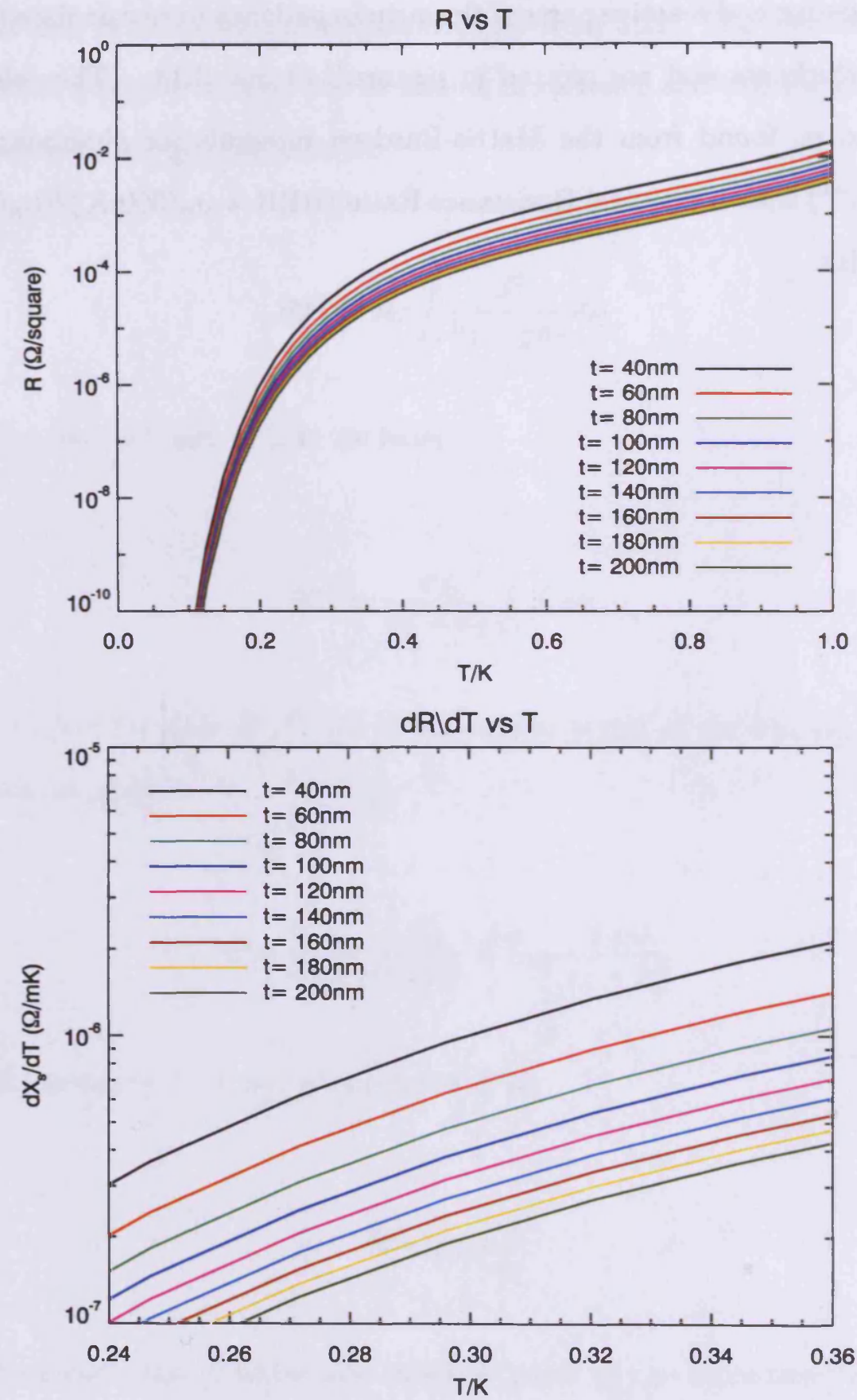

Figure 3.13: (Top) Resistance vs temperature in $\Omega$ /square of a superconducting aluminium strip at at $7 \mathrm{GHz}$ for varying film thickness. As we shall see later the resistance of a strip restricts the unloaded quality factor so we will want this value to be as low as possible. This will require us working at the lowest temperatures achievable by our system. (Bottom) The change in strip resistance with temperature given in $\Omega / \mathrm{mK}$ about the typical base temperature of a typical ${ }^{3} \mathrm{He}$ sorption cooler. 

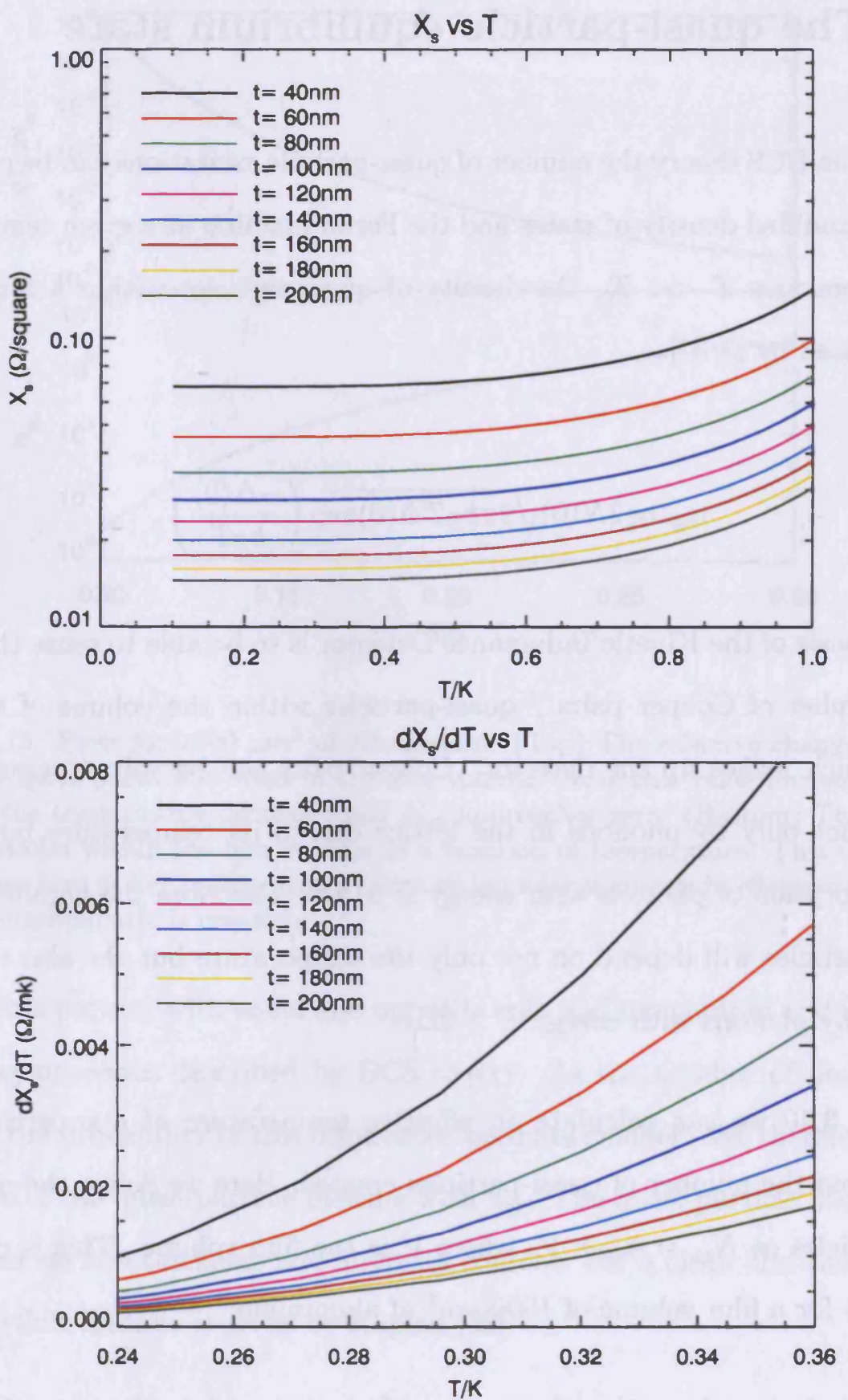

Figure 3.14: (Top) Reactance vs temperature in $\Omega$ /square of a superconducting aluminium strip at at $7 \mathrm{GHz}$ for varying film thickness. (Bottom) The change in reactance with temperature over a small range. The key point here is that although from the top plot the reactance at low temperatures seems to be non-changing this is not the case. Hence a small increase in temperature will indeed change the reactance of a superconducting thin film. Also it is worth noting that the largest change in $X_{s}$ with $\mathrm{T}$ is seen for the thinnest strips. 


\subsection{The quasi-particle equilibrium state}

As seen from BCS theory the number of quasi-particle excitations can be calculated from the modified density of states and the Fermi function at a given temperature. For temperatures $T \ll T_{c}$, the density of quasi-particles within a film can be approximated by $[3,37]$ :

$$
n_{q p}=2 N(0) \sqrt{2 \pi k_{B} T \Delta(0)} \exp \left(\frac{-\Delta(0)}{k_{B} T}\right)
$$

The basis of the Kinetic Inductance Detector is to be able to sense the change in the number of Cooper pairs / quasi-particles within the volume of superconductor which makes up the detector. Cooper pairs can be split to create quasiparticles not only by phonons in the lattice due to its temperature but also by direct absorption of photons with energy $E>2 \Delta$. Therefore the number of pairs / quasi-particles will depend on not only the temperature but the also the power absorbed by photons with energy $E>2 \Delta$.

From 3.40 we can calculate an effective temperature of a superconducting volume from the number of quasi-particles created. Here we define the number of quasi-particles as $N_{q p}=n_{q p} \times V$, where $V$ is the film volume. This is plotted in figure 3.15 for a film volume of $1000 \mu \mathrm{m}^{3}$ of aluminium.

Under photon absorption the number of quasi-particles will rise until an equilibrium value is reached where the number of quasi-particles being created from the incident photons per unit time is equal to the number of quasi-particles recombining to form Cooper pairs. This equilibrium value is set by the quasi-particle lifetime $\left(\tau_{q p}\right)$. For a quasi-particle to recombine to form a Cooper pair it must 


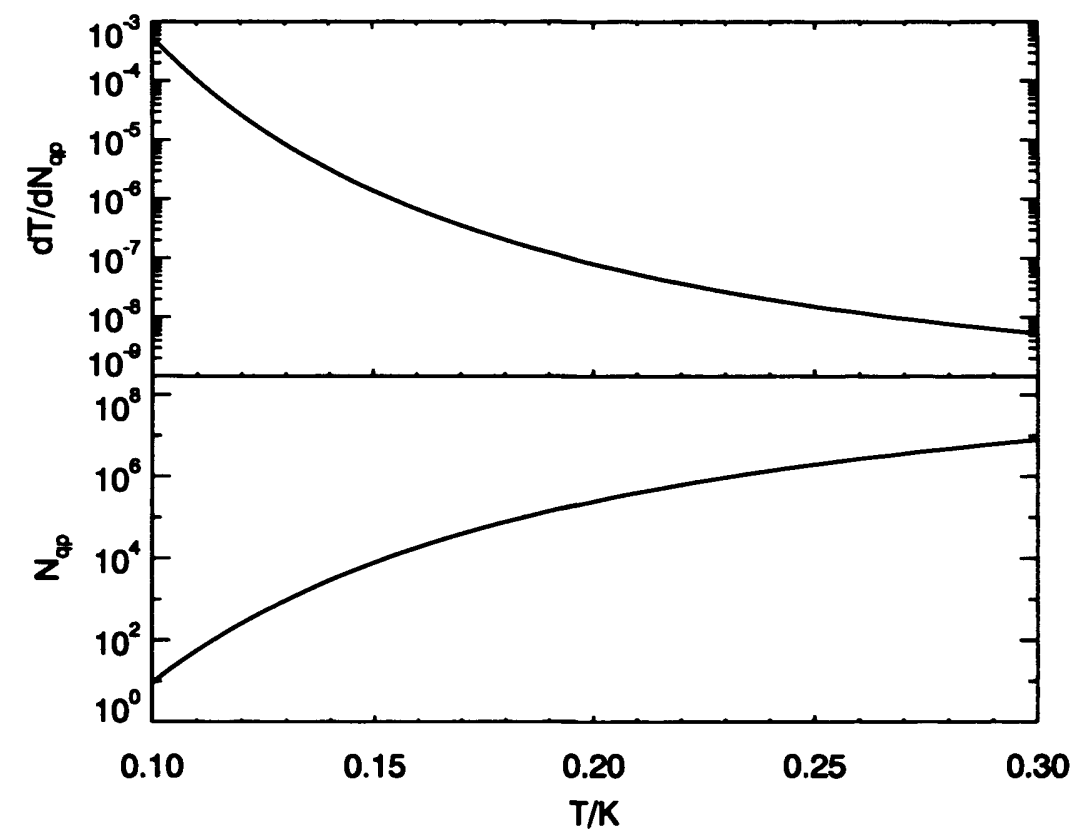

Figure 3.15: Plots for $1000 \mu m^{3}$ of Aluminium. (Top) The effective change in temperature per quasi-particle created in the film volume. Note this value increases exponentially as the temperature decreases and $N_{q p}$ approaches zero. (Bottom) The number of quasi-particles within the film volume as a function of temperature. This value rapidly approaches zero at low temperatures hence giving a large change in effective temperature when a quasi-particle is created.

encounter a partner with equal and opposite spin and momentum and undergo the scattering processes described by BCS theory. As the number of quasi-particles reduces, the probability of this occurrence becomes smaller. We therefore see a dependance of the quasi-particle lifetime with $n_{q p}$. The quasi-particle lifetime is also dependent on film thickness and impurity content. For a clean film the theoretical quasi-particle lifetime is given by Kaplan [38]:

$$
\frac{1}{\tau_{q p}}=\frac{\pi^{\frac{1}{2}}}{\tau_{0}}\left(\frac{2 \Delta}{k_{B} T_{c}}\right)^{\frac{5}{2}}\left(\frac{T}{T_{c}}\right)^{\frac{1}{2}} \exp \left(\frac{-\Delta}{k_{B} T}\right)
$$

Here $\tau_{0}$ is a material dependent quantity set by the electron-phonon coupling strength. Kaplan gives the following values for $\tau_{0}$ : 
Table 3.1: $\tau_{0}$ values for various superconductors

\begin{tabular}{ccc}
\hline \hline Metal & $T_{c} / K$ & $\tau_{0} \times 10^{9} s$ \\
\hline Aluminium & 1.19 & 438 \\
Tantalum & 4.48 & 1.78 \\
Niobium & 9.2 & 0.149 \\
Tin & 3.75 & 2.3 \\
Zinc & 0.875 & 780 \\
\hline
\end{tabular}

The equilibrium excess number of quasi-particles can be estimated by considering a film volume under loading from a continuous flux of photons with $E>2 \Delta$.

$$
N_{x s}=\frac{\eta P \tau_{q p}}{\Delta}
$$

Here $N_{x s}$ is the excess number of quasi-particles in the system, $P$ is the power of the incident photon flux and $\eta$ is an efficiency factor. We will revisit this concept later when looking at the detector responsivity. 


\section{Chapter 4}

\section{Resonator theory and the KID}

\section{concept}

Kinetic Inductance Detectors (KIDs) work on the principle of photons altering the properties of a superconducting microwave resonator. To fully understand this principle and to be able to design an optimum KID we need to have a clear understanding of superconducting resonance structures. We will begin by looking at a simple lumped element resonator.

\subsection{The parallel lumped resonator}

We will start with the analysis of the parallel lumped resonator. By lumped we mean made from components whose physical size is much smaller than a wavelength. The parallel lumped resonator is simply a capacitor $(C)$, an inductor $(L)$ and a resistor $(R)$ in a parallel circuit and is pictured in figure 4.1 


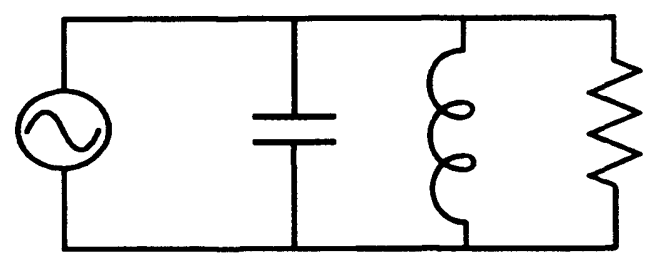

Figure 4.1: The Parallel Lumped Resonator. Here the supply has zero impedance

Resonance is defined as the point when the average energy stored in the inductor $\left(W_{m}\right)$ and the average energy stored in the capacitor $\left(W_{e}\right)$ are equal. This can be calculated by simply equating these energies [39]:

$$
\begin{array}{ccl}
W_{m}=\frac{1}{4} L|I|^{2} & \text { and } & W_{e}=\frac{1}{4} C|V|^{2} \\
W_{m}=W_{e} & \text { when } & \frac{1}{4} L|I|^{2}=\frac{1}{4} C|V|^{2} \\
\Rightarrow & \frac{1}{4} \frac{|V|^{2}}{\omega^{2}} L=\frac{1}{4}|V|^{2} C \\
\Rightarrow & \omega_{0}=\frac{1}{\sqrt{L C}}
\end{array}
$$

where $I$ and $V$ are current and voltage respectively.

Another important parameter to define in a resonant circuit is the quality factor $(Q) . Q$ is defined as:

$$
Q=\omega_{0} \frac{\text { average energy stored }}{\text { average energy dissipated }}
$$




$$
\Rightarrow Q=\omega_{0} \frac{W_{m}+W_{e}}{P_{l}}
$$

$P_{l}$ in this case is the average power dissipated in $R$. The power dissipated in $R$ is simply $|V|^{2} / R$. Since the voltage across $R, L$ and $C$ is the same in a parallel circuit, we can write $W_{e}+W_{m}$ as $2 W_{e}$. Now writing $W_{e}$ in terms of $V$ and $C$ as in equation 4.1 we can write $Q$ in terms of $R$ and $C$ :

$$
Q=\omega_{0} \frac{2 W_{e}}{|V|^{2} / R}=\omega_{0} \frac{\frac{1}{2} C|V|^{2}}{\frac{1}{2}|V|^{2} / R}=\omega_{0} R C=\frac{R}{\omega_{0} L}
$$

It is useful to define an impedance for the resonator to enable us to study its behavior in a circuit. The impedance of the parallel resonator is defined by:

$$
Z_{\text {in }}=\left(\frac{1}{j \omega L}+j \omega C+\frac{1}{R}\right)^{-1}
$$

By considering frequencies near resonance and writing $\omega$ as $\omega_{0}+\Delta \omega$ we can use a Taylor expansion to write the impedance as:

$$
\begin{aligned}
\frac{1}{Z_{\text {in }}} & =\frac{1}{R}+\frac{1}{j\left(\omega_{0}+\Delta \omega\right) L}+j\left(\omega_{0}+\Delta \omega\right) C \\
& =\frac{1}{R}+\frac{1}{j \omega_{0} L}\left(\frac{1}{1+\Delta \omega / \omega_{0}}\right)+j \omega_{0} C+j \Delta \omega C \\
& \approx \frac{1}{R}+j \frac{\Delta \omega L C}{L}+j \Delta \omega C \\
& \approx \frac{1}{R}+2 j \Delta \omega C .
\end{aligned}
$$


The derivation in 4.5 substitutes $1 / \omega_{0}^{2}$ for $L C$.

If we now define $\Delta \omega$ as a fractional shift from $\omega_{0}\left(x=\Delta \omega / \omega_{0}\right)$ and recalling equation 4.3 we can write the impedance around resonance in terms of $R$ and $Q$ as:

$$
Z_{\text {in }} \approx \frac{R}{1+2 j \Delta \omega R C}=\frac{R}{1+2 j Q x}
$$

So far the $Q$ for the circuit we have derived is defined by the properties of the components it is made from, i.e. $L, R$ and $C$, and is known as the unloaded $Q$ $\left(Q_{u}\right)$. We will refer to it as this from here on in. However, the effect of connecting the resonator to any external circuitry is to lower the overall $Q$. This new value is referred to as the loaded $Q\left(Q_{L}\right)$. An example of this loading is shown in figure 4.2 .

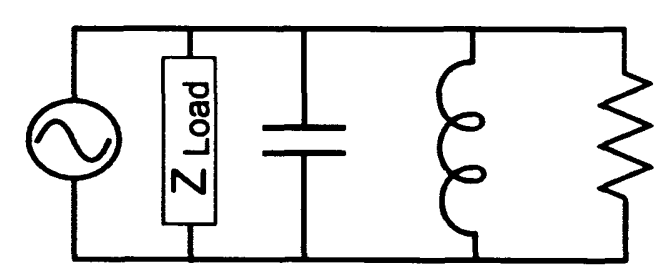

Figure 4.2: Example of external circuitry loading a parallel resonator. This is a more realistic situation of a parallel resonator being driven by a supply with an impedance $Z_{\text {Load }}$

In the case of the parallel resonator the load adds in parallel with the resonator resistance. We can define the effective $R$ as $R / / Z_{L}$. We can now write $Q_{L}$ as:

$$
Q_{L}=\omega_{0} C\left(\frac{1}{R}+\frac{1}{R_{L}}\right)^{-1}
$$




\subsection{The series lumped resonator}

The previous section characterises a lumped resonator in a parallel configuration, however, there are some differences when dealing with a series resonator which are worth noting as they will become useful later in describing the workings of the Lumped Element KID. The basic series resonator is shown in figure 4.4.

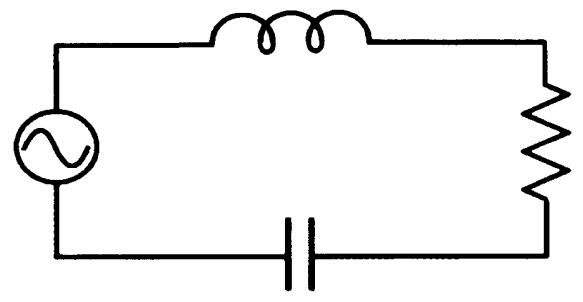

Figure 4.3: The series resonator being driven with a supply with zero impedance.

The basic definition of the resonance frequency of the series resonator is the same as that for the parallel resonator and remains as $\omega_{0}=1 / \sqrt{L C}$. We can define $\mathrm{Q}$ in the same way as we did for the parallel case but defining the power loss as $|I|^{2} R$ in this case as it is the current that is common to each component in the series case. Defining the total average energy stored as $2 W_{L}$ we can write $Q_{u}$ as:

$$
Q_{u}=\frac{2 W_{L}}{|I|^{2} R}=\frac{\frac{1}{2} L|I|^{2}}{\frac{1}{2}|I|^{2} R}=\omega_{0} \frac{L}{R}=\frac{1}{\omega_{0} R C}
$$

The definition of the impedance around resonance can also be derived in a similar manner to that of the parallel resonator as follows: 


$$
\begin{aligned}
Z_{i n}=R+j \omega L+\frac{1}{j \omega C} \quad & =R+j \omega L\left(1-\frac{1}{\omega^{2} L C}\right) \\
& =R+j \omega L\left(\frac{\omega^{2}-\omega_{0}^{2}}{\omega^{2}}\right) .
\end{aligned}
$$

Here we have used the definition of $\omega_{0}^{2}=1 / L C$. Taking advantage of the fact that $\omega^{2}-\omega_{0}^{2}=\left(\omega-\omega_{0}\right)\left(\omega+\omega_{0}\right)=\Delta \omega(2 \omega-\Delta \omega) \approx 2 \omega \Delta \omega$ for small $\Delta \omega$ we can write $Z_{\text {in }}$ as:

$$
\begin{aligned}
Z_{i n} & \approx R+j 2 L \Delta \omega \\
& \approx R+j \frac{2 R Q_{u} \Delta \omega}{\omega_{0}}
\end{aligned}
$$

Connecting the series resonator to an external load has the same effect as in the parallel case, lowering the measured value of $\mathrm{Q}$ to $Q_{L}$. In this case the load adds in series to $R$ and $Q_{L}$ is calculated by:

$$
Q_{L}=\omega_{0} \frac{L}{R+R_{L}}
$$

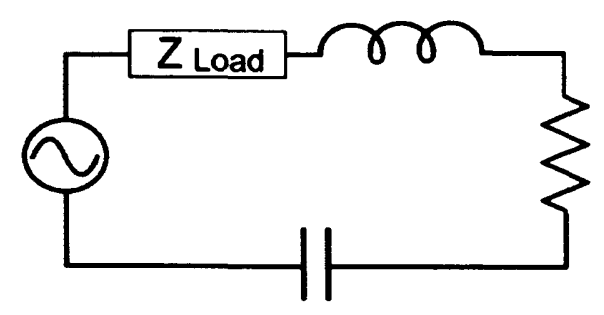

Figure 4.4: The series resonator being driven with a supply with impedance $Z_{\text {load }}$. 


\subsection{The quarter wave resonator}

The preferred geometry for KID devices has been the Quarter Wave resonator [2,3]. This device consists of a length of transmission line shorted at one end and capacitively coupled to a second transmission line from which the resonator is excited. Both sections of transmission line are designed to have the same characteristic impedance $Z_{0}$ (usually $50 \Omega$ ) by setting the ratio of center strip to gap width the same in both sections. A schematic of the quarter wave resonator is shown in figure 4.5 .

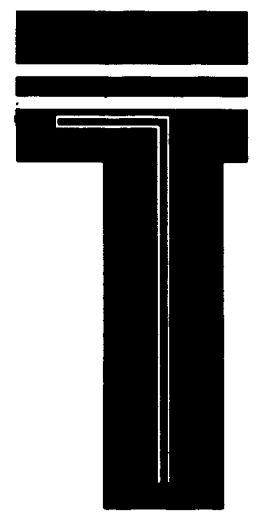

a
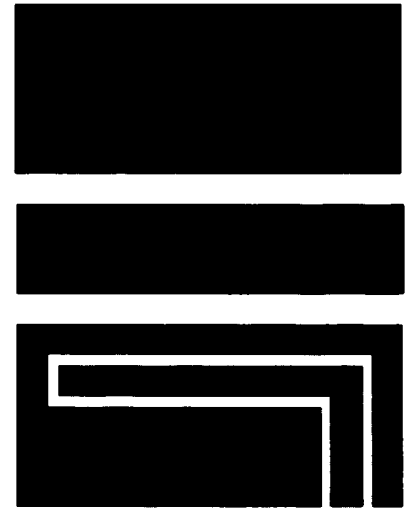

b

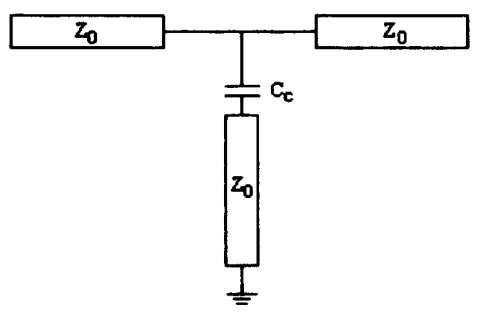

c

Figure 4.5: (a) Schematic of the Quarter Wave resonator using a coplanar waveguide geometry. Here both the sections of transmission line are designed to have the same characteristic impedance $Z_{0}$. (b) A close up on the capacitive coupling section. (c) The equivalent circuit model.

The device will resonate at a wavelength of $4 l$ where $l$ is the length of the quarter wave section. We can derive the impedance of the quarter wave section by following the example set out by Pozar [40]. Here the impedance is written as: 


$$
Z_{\text {in }}=Z_{0} \frac{1-j \tanh (\alpha l) \cot (\beta l)}{\tanh (\alpha l)-j \cot (\beta l)}
$$

where $\alpha$ is the attenuation constant of the line and $\beta=2 \pi / \lambda$ is the wave number. Now if we say that $l=\lambda / 4$ at $\omega=\omega_{0}$ then for a TEM transmission line we can say:

$$
\beta l=\frac{\omega_{0} l}{v_{p}}+\frac{\Delta \omega l}{v_{p}}=\frac{\pi}{2}+\frac{\pi \Delta \omega}{2 \omega_{0}}
$$

where $v_{p}$ is the phase velocity on the line, hence:

$$
\cot (\beta l)=\cot \left(\frac{\pi}{2}+\frac{\pi \Delta \omega}{2 \omega_{0}}\right)=-\tan \left(\frac{\pi \Delta \omega}{2 \omega_{0}}\right) \approx \frac{-\pi \Delta \omega}{2 \omega_{0}}
$$

Here we have used the cofunction identity $\cot (\pi / 2+x)=-\tan (x)$ and $\tan (x) \approx$ $x$ for small values of $x$. Using the fact that $\tanh (x) \approx(x)$ for small $x$ we can write equation 4.12 as:

$$
Z_{\text {in }}=Z_{0} \frac{1+j \alpha l \pi \Delta \omega / 2 \omega_{0}}{\alpha l+j \pi \Delta \omega / 2 \omega_{0}} \approx \frac{Z_{0}}{\alpha l+j \pi \Delta \omega / 2 \omega_{0}}
$$

as $j \pi \Delta \omega / 2 \omega_{0}<<1$. By dividing the top and bottom of the right hand side of equation 4.15 by $Z_{0}$, we arrive at a result which takes the same form as equation 4.6 (the parallel lumped resonator). We can use this comparison to form an equivalent circuit model for the quarter wave resonator made up of lumped components giving an input impedance of: 


$$
Z_{\text {in }}=\frac{1}{\left(\alpha l / Z_{0}\right)+2 j \Delta \omega C_{l i n e}}
$$

Here we have used $1 /\left(\omega_{0} Z_{0}\right)=C$ and defined the capacitance as $C_{\text {line }}$ to make it clear we are dealing with the capacitance in the quarter wave transmission line section.

Comparison with equation 4.6 shows that we can equate $\alpha$ to an equivalent resistance of the resonator by:

$$
R=\frac{Z_{0}}{\alpha l}
$$

We can also derive an equivalent capacitance for the resonator by comparing the $2 j \Delta \omega / \omega_{0}$ term in equation 4.15 to the $2 j \Delta \omega C_{\text {line }}$ term in equation 4.16 giving:

$$
C_{\text {line }}=\frac{\pi}{4 \omega_{0} Z_{0}} \text { thus } \quad L_{\text {line }}=\frac{4 Z_{0}}{\omega_{0} \pi}
$$

Now noting that on resonance the wavelength is equal to $4 l$, from equation 4.13 , we can write $\beta=\pi / 2 l$. This allows us to define a $Q_{u}$ for the circuit in the same manner as for the parallel lumped resonator:

$$
Q_{u}=\omega_{0} R C_{\text {line }}=\frac{\pi}{4 \alpha l}=\frac{\beta}{2 \alpha}
$$

We can substitute this result back in to equation 4.15 to give the impedance 
in terms of $Q_{u}$ :

$$
Z_{i n}=\frac{Z_{0}}{\pi / 4 Q_{u}+j \pi \Delta \omega / 2 \omega_{0}}=\frac{Z_{0} 4 Q_{u} / \pi}{1+j 2 Q_{u} \Delta \omega / \omega_{0}}
$$

The quarter wave resonator is coupled to the feed line though a capacitive impedance created by running a section of the quarter-wave resonator close to the feedline. The impedance of the entire resonance section includes this coupling capacitance which we will denote as $C_{\text {couple }}$. We can now write the impedance looking in to the entire resonant section as:

$$
Z_{\text {in }}=\frac{1}{j \omega C_{\text {couple }}}+\frac{Z_{0} 4 Q_{u} / \pi}{1+j 2 Q_{u} \Delta \omega / \omega_{0}}
$$

As before resonance is defined when $Z_{\text {in }}$ is real, the addition of the coupling capacitor modifies the resonant condition and reduces the resonant frequency. The extent of this effect can be calculated by looking at frequencies where equation 4.21 is purely real. This calculation leads to the result set out by Mazin [3]:

$$
\omega_{0}-\omega_{1 / 4}=\frac{-2 Z_{0} \omega_{1 / 4}^{2} C_{c o u p l e}}{\pi}
$$

Here $\omega_{1 / 4}$ is the quarter wave resonance condition and $\omega_{0}$ is the new resonant frequency modified by the coupling capacitor. This leads to the impedance of the entire resonant section being defined by:

$$
Z_{\text {in }}=\frac{\pi}{4 Z_{0}\left(\omega_{0} C_{\text {couple }}\right)^{2} Q_{u}}\left(1+2 j Q_{u} x\right)
$$


Usually we write the first term in 4.23 as a coupling coefficient $g$ where:

$$
g=\frac{4 Z_{0}^{2}\left(\omega_{0} C_{\text {couple }}\right)^{2} Q_{u}}{\pi}
$$

\subsection{Scattering parameters for resonance circuits}

Microwave circuits are typically characterised in terms of their scattering parameters or $S$ parameters as they are more commonly known. Scattering parameters give the ratio of voltages between ports in an $n$ port microwave system. For a two port system, such as the systems dealt with in this thesis, there are four scattering parameters, $S 11, S 12, S 21$ and $S 22$. These parameters are defined as follows [40]:

$$
\begin{array}{ll}
S 11=\frac{V_{1}^{-}}{V_{1}^{+}} & S 21=\frac{V_{2}^{-}}{V_{1}^{+}} \\
S 12=\frac{V_{1}^{-}}{V_{2}^{+}} & S 22=\frac{V_{2}^{-}}{V_{2}^{+}}
\end{array}
$$

here $V_{n}^{+}$is defined as the voltage wave incident on port $n$ and $V_{n}^{-}$is defined as the voltage wave traveling from port $n$. For example $V_{2}^{-} / V_{1}^{+}$is the ratio of the voltage wave traveling from port 2 to the voltage wave incident on port 1 and defines the transmission through the system in the direction from port 1 to port 2 .

The $S$ parameters for a two port system can be described by the ABCD matrix [40]. For various circuit configurations (series or shunt for example) there is a corresponding $\mathrm{ABCD}$ matrix. The quarter wave resonator is a shunt impedance to ground and has the following ABCD matrix: 


$$
\begin{array}{cc}
A=1 & B=0 \\
C=Y & D=1
\end{array}
$$

where $Y$ is the admittance $\left(1 / Z_{\text {in }}\right)$ of the shunt impedance. From a given $\mathrm{ABCD}$ matrix all four scattering parameters of a two port system can be calculated by:

$$
\begin{array}{ll}
S 11=\frac{A+B Z_{0}-C Z_{0}-D}{A+B / Z_{0}+C Z_{0}-D} & S 12=\frac{2(A D-B C)}{A+B / Z_{0}+C Z_{0}+D} \\
S 12=\frac{2}{A+B / Z_{0}+C Z_{0}+D} & S 22=\frac{-A+B Z_{0}-C Z_{0}-D}{A+B / Z_{0}+C Z_{0}-D}
\end{array}
$$

Using the equations in 4.27 we can calculate the transmission of a microwave signal propagated along the feedline past the resonator. For any practical readout system of a KID device we are only concerned with the forward scattering matrix $(S 21)$. In terms of the quarter wave resonator $S 21$ reduces to:

$$
S 21=\frac{2}{2+Z_{0} / Z_{\text {in }}}
$$

Here $Z_{0}$ is the characteristic impedance of the the feed line and ports, which we will set equal to $50 \Omega$ unless otherwise stated from here on. $Z_{\text {in }}$ is the impedance looking in to the resonant section and is set by equation 4.23. Far from the resonant condition $Z_{\text {in }}$ is very large so $Z_{0} / Z_{\text {in }}$ approximates to zero and the transmission 
is equal to unity. However around resonance $Z_{\text {in }}$ reduces, reaching a minimum on resonance at which point $S 21$ becomes a minimum and is completely real. We can calculate the transmission on resonance by setting $x$ in equation 4.23 to zero:

$$
S 21_{0}=\frac{2}{2+g}
$$

As we move around resonance (i.e. $x$ in equation 4.23 is non zero) S21 becomes complex and hence we observe a shift in the phase of S21. The phase of S21 can be calculated by the complex argument of equation 4.30 . The power transmitted past the resonator if the impedance of both ports are equal is simply $|S 21|^{2}$.

$$
\begin{aligned}
S 21 & =\left(\frac{2}{2+g}\right) \frac{1+2 j Q_{u} x}{(2 / 2+g) 2 j Q_{u} x+1} \\
\rightarrow|S 21|^{2} & =\left(\frac{2}{2+g}\right)^{2} \frac{1+4 Q_{u}^{2} x^{2}}{\left(4 Q_{u} x / 2+g\right)^{2}+1} .
\end{aligned}
$$

As with the series and parallel resonators the quarter wave resonator will have a loaded $Q, Q_{L}$. The loading in the quarter wave case is dictated by the strength of the coupling to the feed line. This is basically set by the value of the coupling capacitor. The stronger the coupling the easier it is for energy to leak from the resonator into the feedline where it is effectively dissipated. We can calculate a value of $Q_{L}$ by calculating the $3 \mathrm{~dB}$ bandwidth of the power transmitted past the resonator. If a resonator is heavily coupled we can use the approximation that $|S 21|^{2} \approx 0$ on resonance. Using equation 4.31 and denoting the transmitted power on resonance as $|S 21|_{0}^{2}$, we can find values for $x$ where the transmitted power is 
equal to a half thus giving:

$$
\begin{gathered}
|S 21|^{2}=\frac{1}{2}\left(|S 21|_{0}^{2}+1\right) \text { when } \frac{1+4 Q_{u}^{2} x^{2}}{1+\left(4 Q_{u} x / g+2\right)^{2}}=\frac{1}{2}+\frac{1}{2}\left(\frac{g+2}{2}\right)^{2} \\
\rightarrow 4 Q_{u}^{2} x^{2}=\frac{1-(g+2 / 2)^{2}}{(2 / g+2)^{2}-1}=\left(\frac{g+2}{2}\right)^{2} \\
\rightarrow x=\frac{1}{2 Q_{u}}\left(1+\frac{1}{2} g\right)
\end{gathered}
$$

Now we can define $Q_{L}$ as $\omega_{0}$ divided by the $3 \mathrm{~dB}$ bandwidth, which, from equation 4.33 is $2 \omega_{0} x$ therefore $Q_{L}$ is given as

$$
\begin{aligned}
Q_{L} & =Q_{u}\left(1+\frac{1}{2} g\right)^{-1} \\
\rightarrow Q_{L} & =Q_{u}|S 21|_{0} .
\end{aligned}
$$

Equation 4.35 is very useful for determining $Q_{u}$ and hence some idea of the film quality.

\subsection{Principles of operation of a KID}

So far we have derived the equations for the surface impedance of a superconducting film and for characterising the quarter wave resonator. We will now move on to 
use the variation of surface impedance with Cooper pair density in conjunction with a quarter wave resonator to demonstrate a photon detector. The resonant wavelength in a quarter wave resonator is fixed by the length of the resonator $l$. However the resonant frequency $\omega_{0}$ is set by the phase velocity $v_{p}$. The phase velocity for any TEM transmission line is given by:

$$
v_{p}=\frac{\omega}{\beta}=\frac{1}{\sqrt{L_{\text {line }} C_{\text {line }}}}
$$

The fundamental principal behind the quarter-wave KID is that $L_{\text {line }}$ is not fixed but is in fact made up from the geometric inductance $\left(L_{e x t}\right)$ and the internal inductance $\left(L_{i n t}\right)$ which, for a fixed geometry is inversely proportional to the Cooper pair density $n_{s}$. Therefore a change in internal inductance will modify the quarter-wave condition leading to a new resonant frequency. The shift in resonant frequency will depend on the change in total inductance $L_{t o t}$. $L_{\text {ext }}$ is fixed by the geometry of the line and so the change in $L_{\text {tot }}$ is proportional to fractional change in $L_{\text {int }}$ to $L_{\text {tot }}$. It is therefore useful to define the ratio of internal inductance to total inductance as $\alpha$ :

$$
\alpha=\frac{L_{\text {int }}}{L_{\text {tot }}}
$$

It should now be clear that as Cooper pairs are broken in the resonator, there is an increase in internal inductance, and hence $L_{t o t}$. The increase in $L_{\text {tot }}$ will reduce the phase velocity in the line which in turn will reduce the resonant frequency. This shift in resonant frequency is proportional to the change in total inductance, hence for a large change in resonant frequency with change in internal inductance we want $\alpha$ to be as large as possible. 
Cooper pairs can be broken by absorption of a photon with energy $E>2 \Delta$. This means we can alter the resonant frequency of the distributed KID simply by shining light on it; hence it acts as a photon detector. The current distribution in the distributed KID is non-uniform along its length, being zero at the open end and a maximum at the shorted end. The current evolves along the length of the line as $I_{l o c}=\sin (\pi z / 2 l)$ where $I_{l o c}$ is the local current normalised to the maximum current on the line, $z$ is the position on the line and $l$ is the line length. This condition leads to a position dependent response of the distributed KID to a pair breaking event. For example, a Cooper pair broken in a region where there is no current will have no effect on the resonant frequency. For this reason pair breaking events due to photon absorption should be confined to the shorted end of the KID where the current is at a maximum [3]. Coplanar structures also demonstrate a non uniform current distribution across the width of the centre strip and ground planes with the current being at a maximum at the edges of each [41]. This tends not to give any position dependance, as a quasi-particle, once generated can diffuse very long distances (hundreds of microns) $[6,42]$, compared to the relatively short width of the centre strip (typically $<5 \mu m$ ). This invariably means that once a quasi-particle is created it will diffuse into the high current density region. Figure 4.6 shows the current distribution across the width of a typical coplanar line.

\subsection{The lumped element KID}

To date most efforts in developing KID devices have concentrated on the quarter wave resonator. However work has been carried out in the detector group at Cardiff to develop a lumped element superconducting resonator for use as a photon detector. Such a device, unlike the quarter wave resonator has a far more uniform 


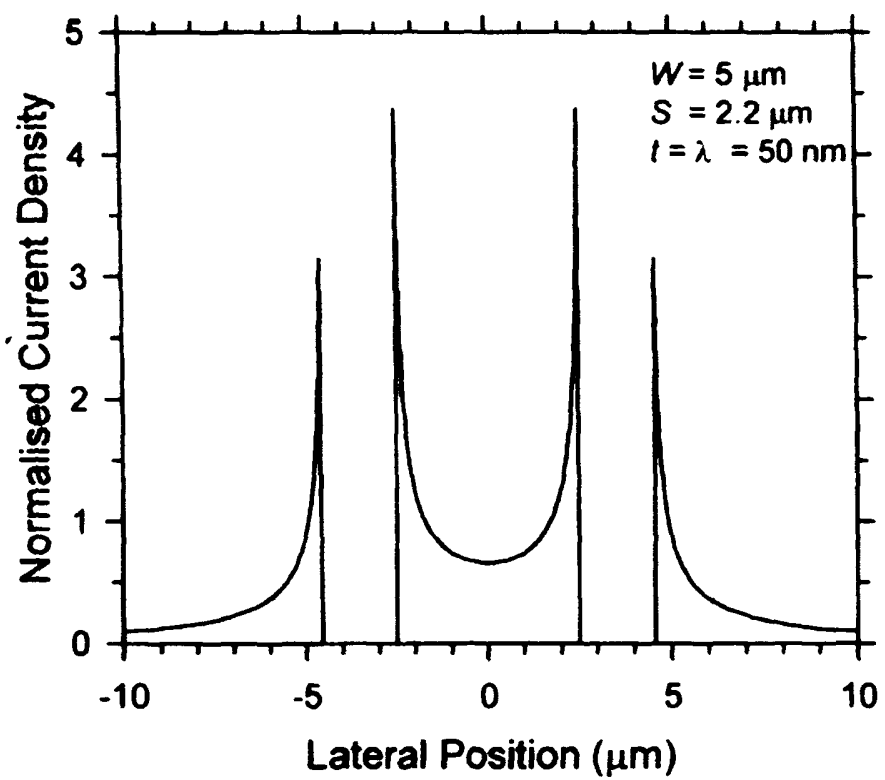

Figure 4.6: Simulated current density on the top surface of a typical coplanar line normalised by the average current density [41].

current distribution and is physically smaller. If a resonator has a uniform current distribution it means that the shift in resonant frequency due to a pair breaking event will be the same regardless of where that event takes place. This has the advantage of not having to direct the photons to be detected to a specific point on the resonator but just allow them to fall naturally on to it much as they would do in the case of a CCD. The Lumped Element Kinetic Inductance Detector (LEKID), combines the properties of being a high $\mathrm{Q}$ superconducting resonator being sensitive to pair braking events, and an absorbing element capable of coupling to an incoming photon.

As a superconducting resonator the basic concept of the LEKID is to inductively couple a series LC circuit to a superconducting microstrip feedline with a superconducting ground-plane. A basic schematic and equivalent circuit model of the LEKID is shown in figure 4.7. The inductive meander acts as absorbing as well as sensing element and therefore must be of size comparable to or greater than a 
wavelength of the photons to be sensed. The meander section as an absorber will be discussed in detail in chapter 7 , but for now we will concern ourselves with the relevant microwave details of the LEKID.

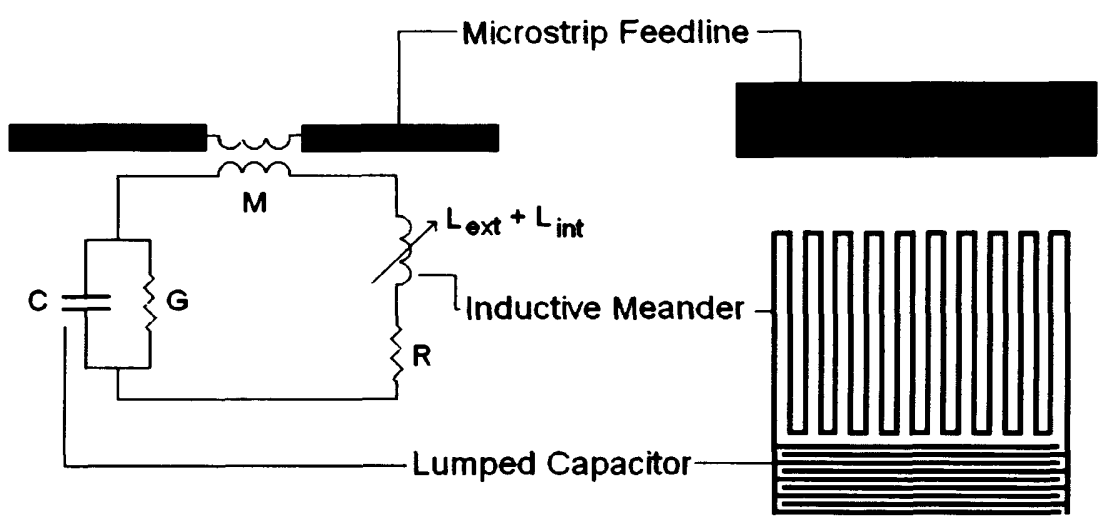

Figure 4.7: (Right) A schematic of a LEKID device. (Left) The equivalent LEKID circuit. Here $M$ is the mutual inductance between the LEKID and the feedline, $R$, is the resistance of the meander section, $L_{e x t}+L_{i n t}$ is the total inductance of the meander section, $C$ is the capacitance of the inter-digital and $G$ is the conductance of the interdigital capacitor section.

The device is etched from a single layer superconducting film deposited on to a low loss substrate such as sapphire. The resonant frequency of the device is simply given by:

$$
\omega_{0} \approx \frac{1}{\sqrt{\left(L_{e x t}+L_{i n t}\right) C}}
$$

where $L$ and $C$ are the inductance and capacitance of the meander and interdigital section respectively. The coupling of the LEKID to the microstrip feedline is inductive and is caused by magnetic flux from the microstrip feedline threading the meander section. To analyse the scattering parameters of such a device it is useful to consider the resonant section as shown in figure 4.8. 


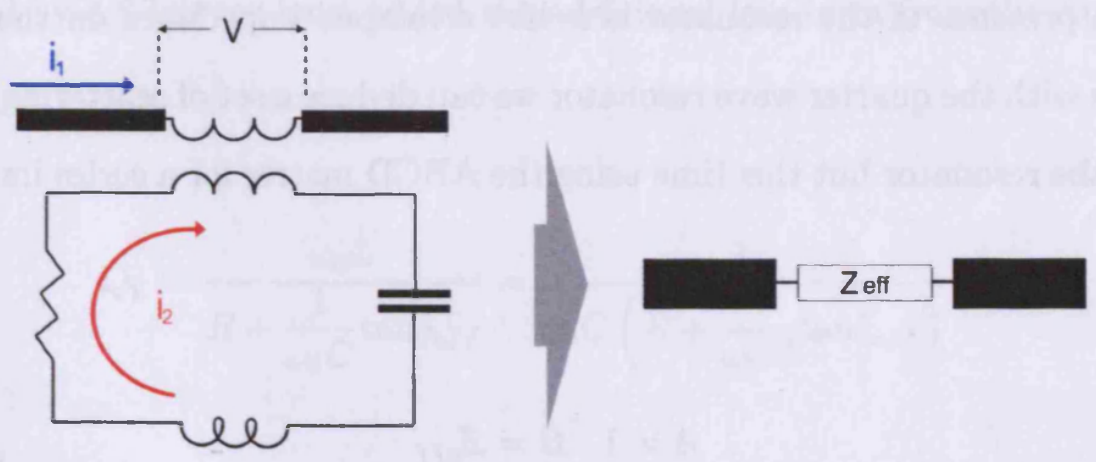

Figure 4.8: The LEKID resonator simplified as a complex impedance $\left(Z_{\text {res }}\right)$ inductively coupled to a feedline with mutual inductance $M$.

Here we show two currents, the current on the feedline $\left(i_{1}\right)$ and the current induced in the resonant circuit $\left(i_{2}\right)$. From the laws of electromagnetic induction we know that the voltage across the coupling section of feedline is $V=j \omega M i_{2}$. From Kirchhoff's laws we know that:

$$
j \omega M i_{1}+i_{2} Z_{r e s}=0 \rightarrow i_{2}=-\frac{j \omega M i_{1}}{Z_{\text {res }}}
$$

and therefore

$$
V=j \omega M \cdot \frac{-j \omega M i_{1}}{Z_{\text {res }}}=\frac{\omega^{2} M^{2} i_{1}}{Z_{\text {res }}}
$$

From equation 4.40 we can deduce the effective impedance the resonant section presents to the line. By definition $Z=V / I$ we can therefore say:

$$
Z_{\text {eff }}=\frac{V}{i_{1}}=\frac{\omega^{2} M^{2}}{Z_{\text {res }}}
$$


The presence of the resonator acts like a lumped impedance on the feedline $Z_{\text {eff }}$. As with the quarter wave resonator we can deduce a set of scattering parameters for the resonator but this time using the ABCD matrix for a series impedance:

$$
\begin{array}{ll}
A=1 & B=Z_{\text {eff }} \\
B=0 & D=1
\end{array}
$$

Using 4.27 we can deduce the forward scattering matrix $S 21$ for a LEKID device:

$$
S 21=\frac{2}{2+\frac{Z_{e f f}}{Z_{0}}}=\frac{1}{1+\frac{\omega^{2} M^{2}}{2 Z_{r e s} Z_{0}}}
$$

$Z_{\text {res }}$ is simply given by the impedance of the resonator:

$$
Z_{r e s}=j \omega L+\frac{1}{j \omega C}+R+\frac{1}{\omega C} \tan \delta_{e f f}
$$

Here $R$ and $(1 / \omega C) \tan \delta_{\text {eff }}$ are loss terms associated with the residual resistance from the quasi-particles and the conductivity of the capacitive section respectively. The derivation of equation 4.44 uses the loss model of a parallel plate capacitor with loss tangent tand. For an inter-digital capacitor the field distribution is not entirely within the substrate, hence, we need to replace $\tan \delta$ with $\tan \delta_{\text {eff }}$ to account for this.

The unloaded $Q\left(Q_{u}\right)$ for the LEKID is the same as that for a series resonator 
governed by 4.8 but we have added the additional loss term from the capacitor.

$$
Q_{u}=\frac{\omega_{0} L}{R+\frac{1}{\omega_{0} C} \tan \delta_{e f f}}=\frac{1}{\omega_{0} C\left(R+\frac{1}{\omega_{0} C} \tan \delta_{e f f}\right)}
$$

As with any resonant circuit we can define a loaded $Q, Q_{L}$. The LEKID is similar to the quarter wave resonator in the way that it is not directly coupled to an external circuit but is de-coupled by a complex impedance, in this case, an inductance. We can define a couple limited $Q$ often defined as an external $Q, Q_{e}$ by looking at the power dissipated through coupling alone. From the law of mutual electromagnetic induction $\left(V=j \omega M i_{2}\right)$, we can write the power dissipated from this induced voltage as:

$$
P_{d i s s}=\frac{V^{2}}{R}=\frac{\omega^{2} M^{2} i_{2}^{2}}{Z_{0}}
$$

Plugging this into the standard equation for $Q$ (equation 4.2) we can obtain a value for $Q_{e}$ :

$$
Q_{e}=\omega_{0} \cdot L i_{2}^{2} \cdot \frac{Z_{0}}{\omega_{0}^{2} M^{2} i_{2}^{2}}=\frac{L Z_{0}}{\omega_{0} M^{2}}
$$

$Q_{L}$ is defined as:

$$
\frac{1}{Q_{L}}=\frac{1}{Q_{u}}+\frac{1}{Q_{e}}=\frac{1}{Q_{u}}+\frac{\omega_{0} M^{2}}{L Z_{0}}
$$


Defining a coupling coefficient in the same way as we did for the quarterwave resonator we find that:

$$
g=\frac{\omega_{0}^{2} M^{2}}{Z_{0} R}=\frac{\omega_{0} M^{2} Q_{u}}{Z_{0} L}
$$

We can now define $Q_{L}$ in terms of $g$ :

$$
\frac{1}{Q_{L}}=\frac{1}{Q_{u}}+\frac{g}{2 Q_{u}}
$$

Using equation 4.49 and 4.10 we can now write $S 21$ from equation 4.43 in terms of $g$ and a fractional shift from resonance $x$ :

$$
S 21=\frac{2}{2+\frac{g}{1+j 2 Q \Delta \omega / \omega_{0}}}=\frac{2}{2+\frac{g}{1+j 2 Q x}}
$$

\subsection{Modelling the LEKID resonator}

In order to design a LEKID for optimum performance we need to be able to model its scattering parameters. We can do this using equations 4.43 and 4.44 and choosing the appropriate values for $Z_{0}, L, C, R, M$ and $\tan \delta_{\text {eff }}$. The problem is measuring and setting these values to start with. $Z_{0}$ can be easily designed from standard microstrip equations to be $50 \Omega$ and for sapphire at low temperatures we can neglect $\tan \delta$. The microwave losses of a film due to resistivity can be estimated from the Mattis-Bardeen integrals and strip impedance equations (equation 3.39). The values of $M, L$ and $C$ have no simple derivation. The LEKID geometry has to 
be a high $Q$ resonator as well as a matched free space absorber. This gives rise to a complex shape to analyse, and altering one parameter invariably will affect others. For example, the amount of flux threading the meander section will depend on the spacing of the meander lines and the distance of the meander from the feedline. Increasing the space between the meander lines will not only affect the mutual inductance between the resonator and the line $(M)$, but the self inductance of the meander section $(L)$ and, as we shall see in Chapter 7, the free space impedance the meander section presents to an incoming electromagnetic wave.

We may consider the meander section of the resonator as being the most important, as it is this section which is responsible for photon absorption and is responsive to a change in kinetic inductance. For this reason it makes sense to design the rest of the resonator around the design of the meander section. The design of the meander will be discussed in the next section but taking a given meander geometry it is possible to extract values for $L, C$ and $M$ from simulations in Sonnet EM which is a planar microwave circuit simulator. The planar circuit design to be simulated is drawn into the Sonnet project editor with the metal types, substrates and ports being specified by the user. Using a given excitation on one port, Sonnet simulates the electromagnetic field interactions across the design by employing a method of moments analysis based on Maxwells equations [43]. The circuit can be simulated over a desired frequency range and across an arbitrary number of ports. A typical Sonnet simulation design is shown in figure 4.9.

The metal type specified in Sonnet can be an ideal conductor (i.e. one with no loss) or a real conductor with a given surface impedance. The surface impedance can be complex (i.e. contain a kinetic inductance term) which is added in $\Omega$ /square for the real part and $\mathrm{pH} /$ square for the complex part [43]. This feature of Sonnet allow us to extract the inductance $L$ and hence the capacitance $C$ of any resonant 


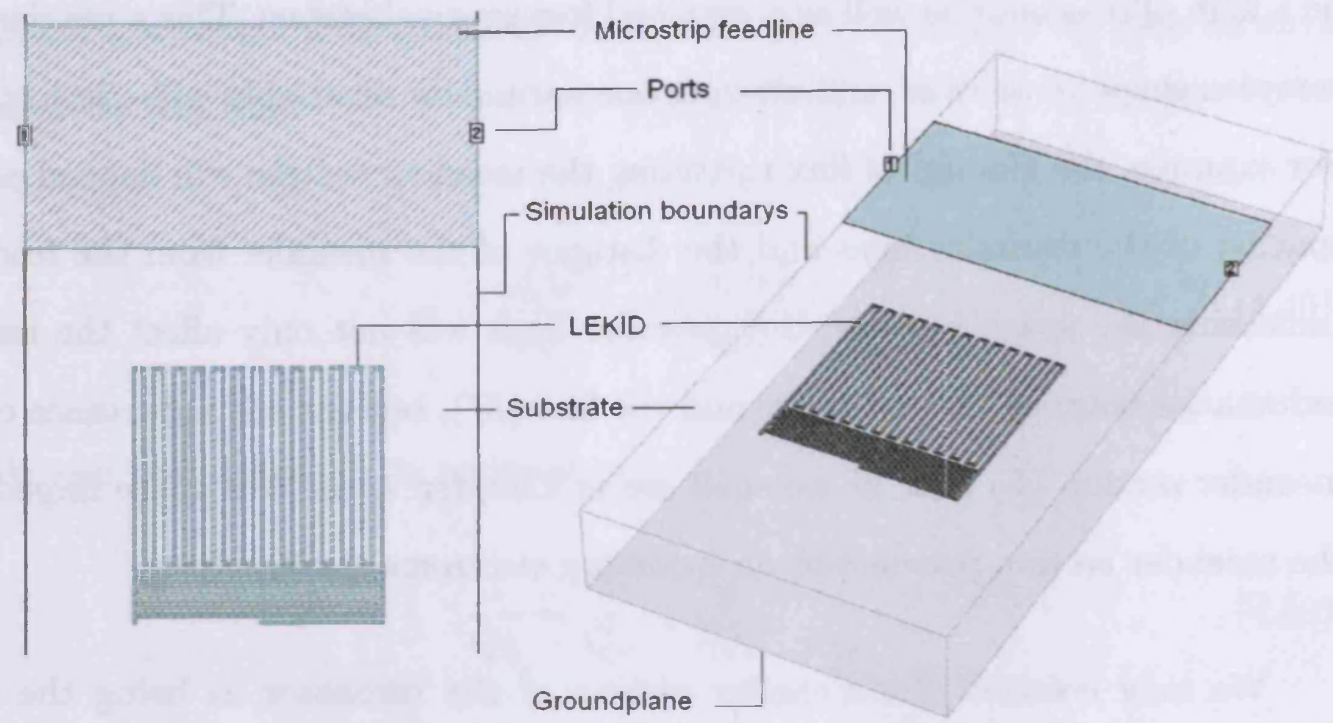

Plan view

$3 D$ view

Figure 4.9: Typical Sonnet layout, here a LEKID resonator has been drawn to simulate its scattering parameters to obtain its resonant frequency $\left(f_{0}\right)$ and coupling coefficient (g). The layout is drawn in 2 dimensions (left) with the substrate thickness making up the third dimension. Metal layers are given zero thickness with a defined impedance in $\Omega /$ square. The simulation is performed in this case between ports 1 and 2 .

structure by performing two simulations. One simulation is performed with no kinetic inductance, hence the resonant frequency is determined by the geometric inductance and the capacitance only. Leaving the layout unchanged but adding kinetic inductance to the simulation will alter the resonant frequency by an amount proportional to $L_{k} / L$. As we can choose the value of $L_{k}$, we can determine the geometrical inductance of the meander section:

$$
\begin{gathered}
\omega_{0}=\frac{1}{L C} \quad \omega_{0}^{\prime}=\frac{1}{\left(L+L_{k}\right) C} \\
\frac{\omega_{0}}{\omega_{0}^{\prime}}=\frac{\sqrt{L+L_{k}}}{\sqrt{L}} \rightarrow\left(\frac{\omega_{0}}{\omega_{0}^{\prime}}\right)^{2}=\frac{L+L_{k}}{L}
\end{gathered}
$$




$$
\rightarrow\left(\frac{\omega_{0}}{\omega_{0}^{\prime}}\right)^{2}=1+\frac{L_{k}}{L} \rightarrow L=\frac{1}{\left(\frac{\omega_{0}}{\omega_{0}^{\prime}}\right)^{2}-1} L_{k}
$$

This method is quite computationally demanding but is necessary to extract a reasonable estimate of the geometric inductance. Simulations were performed on the meander and inter-digital sections alone to try and calculate their individual inductance and capacitance respectively. However we found this method to be inaccurate due to the modifications of the field distributions once these parts were in situ with the feedline and the rest of the resonant structure.

If the geometric inductance and resistance are known, a full simulation of a LEKID resonant element allows us to extract $g$ and hence $M$ for the device by looking at the value of $\mathrm{S} 21$ on resonance. On resonance, $\mathrm{S} 21$ is a minimum and is given by:

$$
S 21_{\min }=\frac{2}{2+\frac{M^{2} \omega^{2}}{Z_{0} R}}=\frac{2}{2+g} \rightarrow g=\frac{2}{S 21_{\min }}-2
$$

As we shall see later the response of any KID device depends on its loaded quality factor. Therefore it is essential to be able to understand and design a value for the mutual inductance which along with $Q_{u}$ governs the loaded quality factor in a LEKID device. 


\section{Chapter 5}

\section{LEKID Design}

As explained in the previous chapter the LEKID resonator needs to be not only a high $Q$ resonant structure but also a matched free-space absorber for incoming photons. Fulfilling both these criteria was not an easy task and required large amounts of effort in microwave simulation. The design process naturally started with choosing a band of radiation to which the detector would be sensitive. The focus throughout this project has been on the mid infrared (MIR) and Far infrared (FIR) corresponding to wavelengths of $5-200 \mu m$. To prove the LEKID concept we decided to design a detector which could absorb $200 \mu \mathrm{m}$ radiation and then look at expanding the idea to incorporate the entire MIR/FIR band.

\subsection{Initial LEKID designs}

For a single pixel to couple to any wavelength efficiently the pixel size needs to be of order of at least a wavelength in size. We decided to start with a pixel size of 
$\approx 250 \mu \mathrm{m} \times 250 \mu \mathrm{m}$ in order to allow a number of electromagnetic modes to exist in the absorption process. The absorption needs to occur in the meander section which will need to be tuned to free space. For the early designs of LEKID devices, this tuning was only weakly addressed as we wanted to demonstrate the LEKID's ability as a high $Q$ resonator. However the early designs were made in such a way that they could be adapted for efficient optical coupling. The main consideration here was the space between the meander lines. In order to make the meander look like a solid sheet to an incoming photon, we needed the spacing to be of order $\lambda / 20$ or less [44]. For this reason the spacing between meander lines was made to be $8 \mu \mathrm{m}$. We decided on a meander line width of $4 \mu \mathrm{m}$. This was set by processing considerations and was thought to be a sensible starting point if we wanted a high yield of resonators to test.

With a meander geometry in place we could begin to design and simulate the rest of the resonator. Two designs were considered for the series capacitor a parallel plated design and an inter-digital design. The parallel plate solution would have comprised a pad deposited and etched on the detector substrate with a dielectric deposited on top separating a second pad forming the other plate of the capacitor. The basic idea of this concept is shown in fig 5.1.

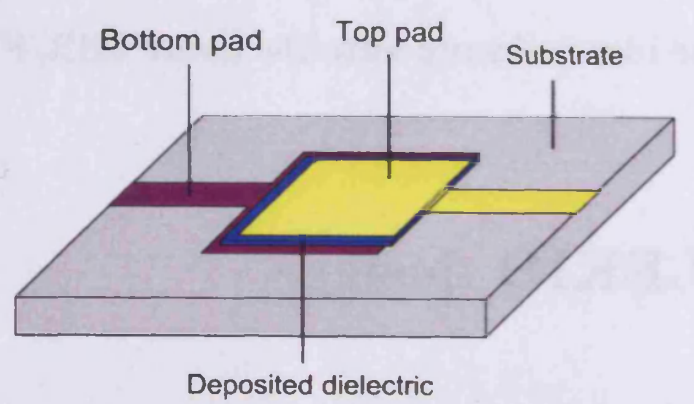

Figure 5.1: Schematic of the parallel plate capacitor 
This idea was disregarded due to losses in the dielectric layer of the capacitor. Deposited dielectrics tend to have high loss tangents due to impurities in the deposition process and two-level systems $[45,46]$. For example thermally evaporated $\mathrm{SiO}_{2}$ has a loss tangent of around $\tan \delta=3 \times 10^{-4}$ at $100 \mathrm{mK}$ [45]. From equation 4.45 we calculate the expected unloaded $Q$ using such a material to be of order $Q_{u}=3 \times 10^{3}$. This low $Q$ factor would suppress the responsivity of our resonators making this approach unsuitable for the LEKID.

The inter-digital capacitor is a planar structure and has a mixed dielectric of sapphire (if used for the detector substrate) and vacuum with the electric field lines being split equally between the two mediums. However, high quality sapphire has a very low loss tangent $\tan \delta \approx 1 \times 10^{-6}$ [45-47]. The loss tangent in sapphire becomes negligible at low temperatures where the losses due to residual resistance in the superconducting film become dominant. A schematic of an inter-digital capacitor is shown in figure 5.2 .

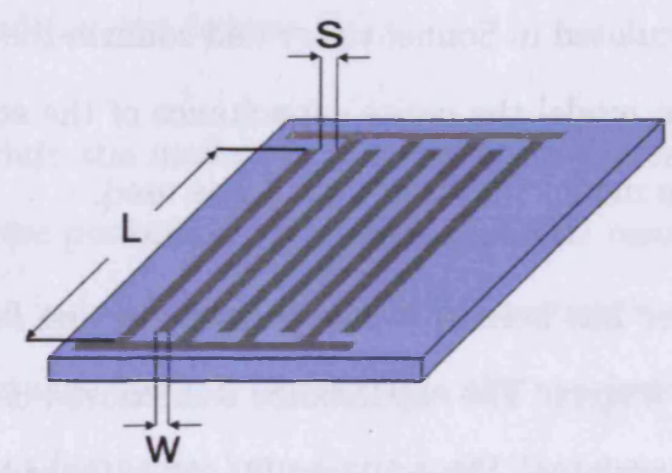

Figure 5.2: Schematic of an 8 finger inter-digital capacitor.

The capacitance is set by the substrate dielectric constant and the dimensions $l, w$ and $s$. To achieve a compact high capacitance inter-digital structure, one would like to make the dimensions $w$ and $s$ as small as possible and use a high 
permittivity substrate. The substrate material is fixed in our case and the length of the inter-digital structure is naturally set by the pixel width. For the first LEKID designs $w$ and $s$ were set to $4 \mu m$ to follow suit with the fabrication tolerances used for the meander section. This leaves us with a single design parameter to consider - the number of fingers used in the inter-digital section. The number of fingers used was set by choosing a resonant frequency. This parameter was bound by the high frequency capabilities of our entire system. This is limited by a $77 \mathrm{~K}$ low noise amplifier (LNA) which had a bandwidth of $4 \mathrm{GHz}$ centred at $6 \mathrm{GHz}$. This sets the range of resonant frequencies to lie between 4 and $8 \mathrm{GHz}$. Like with the meander section, the inter-digital capacitor is difficult to simulate as a lumped component alone. In fact if one looks at the meander section it is clear that there will be some capacitance between the lines of the meander. This 'parasitic' capacitance does not alter the LEKID model we have used so far but we should be aware that it becomes lumped into the inter-digital capacitor when we simulate the entire structure in Sonnet. For this reason a capacitor model as close to the real situation was simulated in Sonnet to try and confirm the values deduced so far for inductance and to model the entire capacitance of the structure as a separate entity. To do this the model shown in fig 5.3 was used.

Here the meander has been split into non-connecting fingers to simulate the capacitance between fingers. The capacitance is measured using the built in equations in the Sonnet simulator. The equation for capacitance is derived from similar ABCD matrix equations explained in Chapter 4 and take the form:

$$
C=\frac{-1}{\omega i m\{Y 11\}}
$$

Here $i m\{Y 11\}$ is the imaginary part of the admittance. The model assumes a 


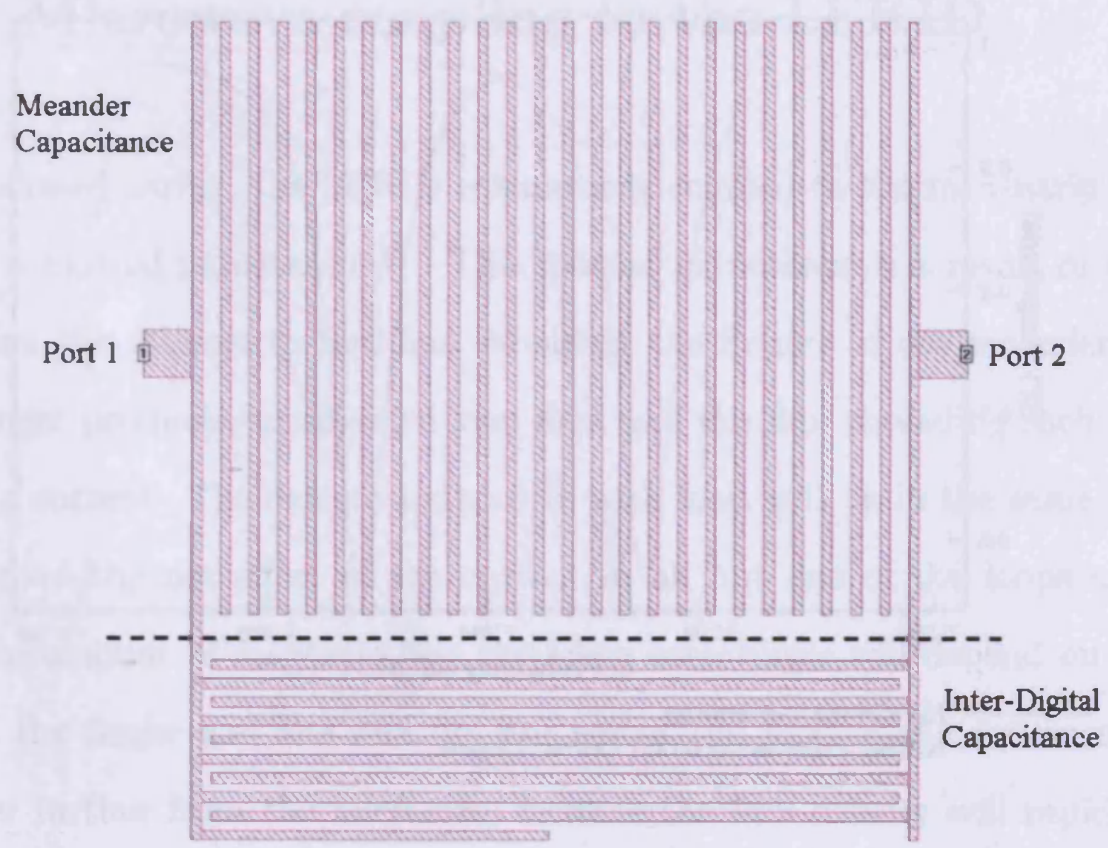

Figure 5.3: Sonnet simulation layout for deducing capacitance of the entire LEKID structure

series capacitance between ports 1 and 2 . For the purpose of the calculation port 2 is shorted to ground (i.e. set to zero $\Omega s$ ).

In order to validate the models used to measure the capacitance and inductance, simulations were performed on an entire LEKID resonator to obtain a resonant frequency. The inductance of the meander section was estimated using the varying kinetic inductance method explained in the previous section. With the inductance and resonant frequency known the capacitance can be calculated simply by using equation 4.38 ; however it was also calculated using the model shown in figure 5.3 to test the robustness of our simulations.

Using equation 4.52 and the data in figure 5.4 we calculate a geometrical inductance for the meander. From the model in figure 5.3 we then deduced a capacitance for the structure in order to check both models. 


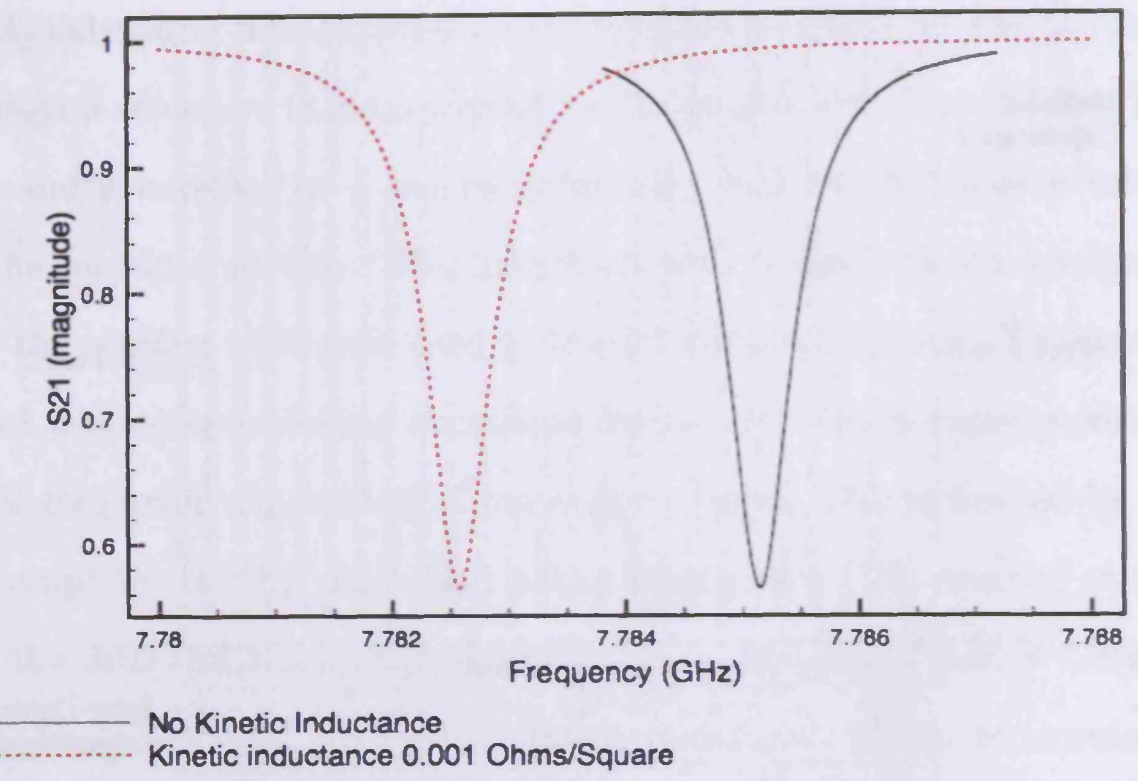

Figure 5.4: Sonnet simulation using a varied kinetic inductance to measure the geometrical inductance from the relative shift in resonance

Table 5.1: Sonnet Simulation Summary

\begin{tabular}{cccc}
\hline \hline Method & Inductance $(\mathrm{nH})$ & Capacitance $(\mathrm{pF})$ & Resonant Frequency $(\mathrm{GHz})$ \\
\hline Lk model & 2.53 & 0.165 & 7.785 \\
Capacitor Model & 2.53 & 0.175 & 7.61 \\
& & $\Delta C=0.01$ & $\Delta f 0=0.175$ \\
\hline
\end{tabular}

Table 5.1 shows the calculated resonant frequency for the two methods of simulating the capacitance of the structure. Method "Lk model" simply uses the calculated inductance to calculate a capacitance from $f_{0}$. Method "Capacitor Model" takes the same value for the inductance and calculates the resonant frequency from the capacitance found from the model shown in fig 5.3. The two methods are in reasonable agreement showing a slight difference in capacitance and hence resonant frequency of $0.01 \mathrm{pF}$ and $0.175 \mathrm{GHz}$ respectively . 


\subsection{Microwave coupling to the LEKID}

As mentioned earlier, the LEKID resonator is coupled to the microstrip feed line though a mutual inductance $M$. This mutual inductance is a result of magnetic field from the microstrip feed-line threading the fingers of the meander section. Each finger produces an effective loop area and the flux threading each loop will induce a current. The current induced in each loop will be in the same direction which gives the net effect of the current in all but one of the loops cancelling out. The amount of magnetic flux threading each finger will depend on the loop area of the finger and the flux density across the loop area. As we move the meander further from the microstrip feedline the flux density will rapidly decay and hence the total flux threading a given loop area will be reduced. This is best demonstrated by approximating the mictrostrip feedline as two current carrying wires. This model uses the assumption that most of the current travels along the edge of a superconducting feedline.

Looking at the model in 5.5 we can calculate the total flux through a given finger by integrating the flux through the volume defined by $d x$ over the length of the finger:

$$
\begin{gathered}
B=\frac{\mu_{0} i / 2}{2 \pi\left(X_{0}+x\right)}+\frac{\mu_{0} i / 2}{2 \pi\left(X_{0}+x+W\right)}, \\
\phi=\int_{0}^{L} \frac{\mu_{0} S i}{4 \pi} d x\left(\frac{1}{X_{0}+x}+\frac{1}{X_{0}+x+W}\right), \\
\phi=\frac{\mu_{0} S i}{4 \pi}\left[\ln \left(X_{0}+x\right)+\ln \left(X_{0}+x+W\right)\right]_{0}^{L},
\end{gathered}
$$




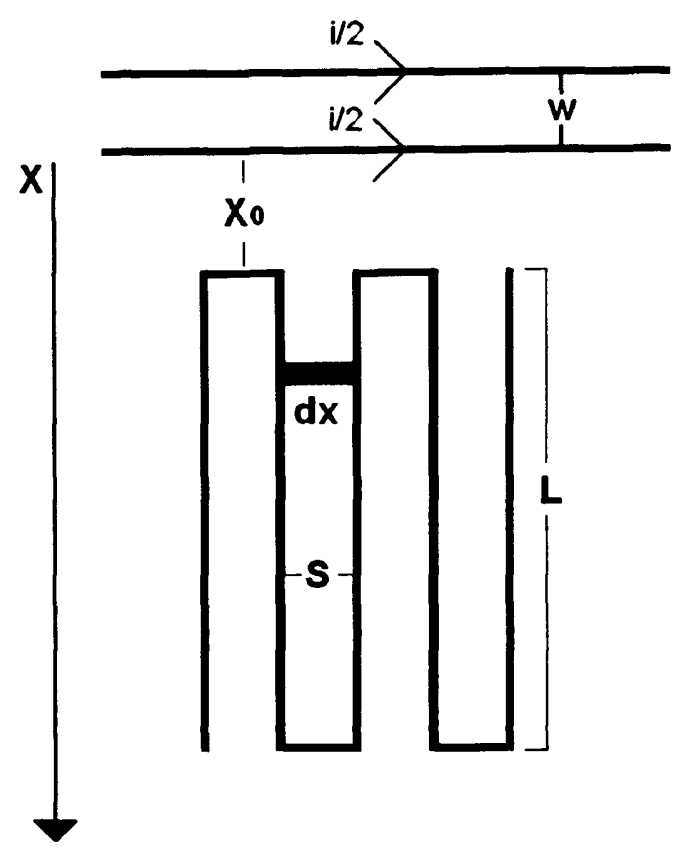

Figure 5.5: Mutual inductance model based on a microstrip feed-line represented by two thin wires

$$
\begin{gathered}
\phi=\frac{\mu_{0} S i}{4 \pi} \ln \left(\frac{(x+L)\left(X_{0}+W+L\right)}{X_{0}\left(X_{0}+W\right)}\right), \\
M=\phi_{2} / I_{1} \\
\rightarrow M=\frac{\mu_{0} S}{4 \pi} \ln \left(\frac{\left(X_{0}+L\right)\left(X_{0}+W+L\right)}{X_{0}\left(X_{0}+W\right)}\right) .
\end{gathered}
$$

The model laid out in figure 5.5 assumes a superposition of two perfectly circular field distributions generated by the currents $i / 2$ in the two wires. This model can be used to give an approximation of the mutual inductance between the LEKID and the microstrip feedline within an order of magnitude but does not work well for the real situation. The addition of a superconducting ground-plane 
and the LEKID itself along with the fact the the microstrip line has a finite width modifies the field distribution considerably. This is illustrated in figure 5.6.

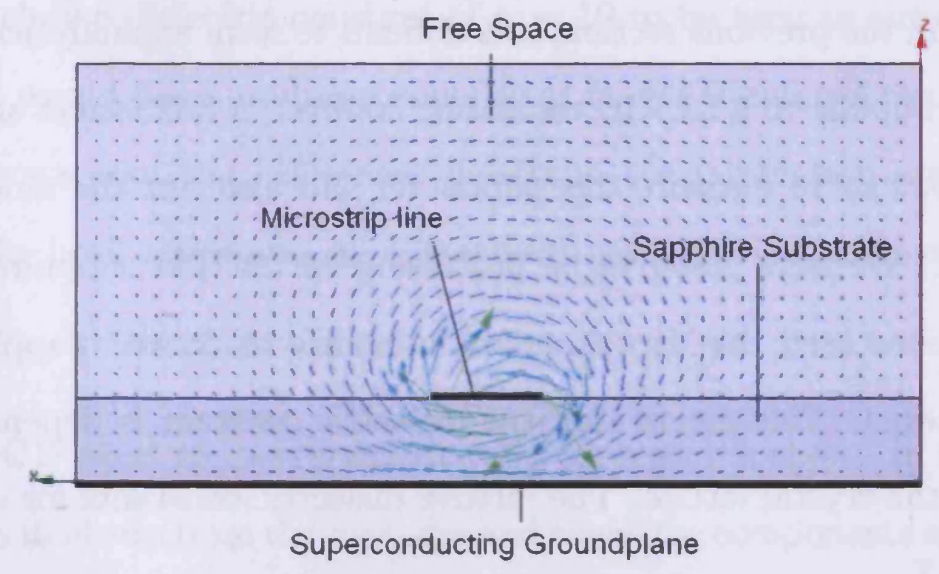

Figure 5.6: HFSS model of the vector B field generated from a current in a superconducting micro-strip line

Figure 5.6 was generated using Ansoft's High Frequency Signal Simulator (HFSS). This figure illustrates two important points not considered in the simple two wire model of figure 5.5. The first is that the field is compressed by the presence of the ground-plane and the microstrip. The second is a modification to the field direction at the substrate surface. The two wire model assumes that the field is perpendicular to the fingers of the meander in the plane of the meander, hence all the flux (i.e. its magnitude at the surface) contributes to an induced current in the meander. The HFSS simulation shows that the perpendicular field at the substrate surface is modified and so a vector summation would need to be introduced to calculate the induced current in the meander. For this reason it is difficult to give an analytical solution for the mutual inductance between the microstrip feedline and the LEKID. To get around this problem a full Sonnet simulation was performed on each LEKID design to estimate the mutual inductance and hence the strength of microwave coupling. 


\subsection{Sonnet simulations of the LEKID}

As mentioned in the previous section, it is difficult to form an analytical model for the magnetic coupling to a LEKID resonator, however, a full Sonnet simulation of the device allows us to measure this parameter and also test the model we have derived so far. We were going to be processing on $325 \mu \mathrm{m}$ sapphire substrates so our simulations used this as a standard substrate thickness. Sapphire is a birefringent material. This means that the dielectric constant is dependent on the orientation of the crystal lattice. The relative dielectric constants for sapphire are shown in fig 5.7 .

A Plane ( $\|$ to $\mathrm{C}$ axis) $\epsilon_{r}=11.6$ $\mathrm{C}$ Plane $(\perp$ to $\mathrm{C}$ axis $) \epsilon_{r}=9.3$

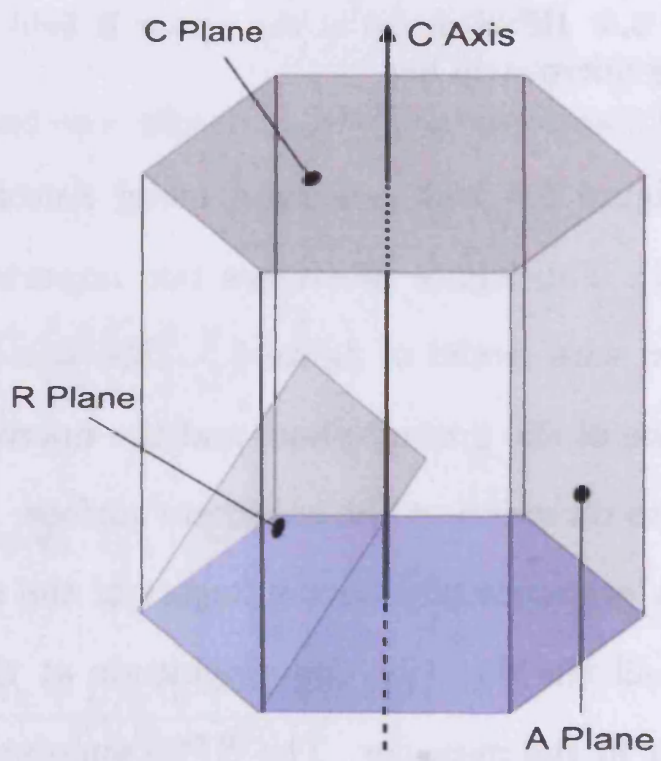

Figure 5.7: Bi-refringent dielectric constants of sapphire. Values given at $300 \mathrm{~K} 10^{3}-$ $10^{10} \mathrm{~Hz}[48]$

We were intending to process our devices on a range of orientations of sapphire and hence a range of different dielectric constants. Sonnet does have the capability of adding bi-refringent substrates, however, these types of simulations tend to be very time consuming and impractical. The change in substrate dielectric constant 
would be seen most in the capacitance of the LEKID and the impedance of a fixed microstrip feedline geometry. These effects were deemed to be small so we decided to take a relative dielectric constant of $\epsilon_{r}=10$ to be used in our simulations. Of course this would have produced some error in the simulated resonant frequency and microwave coupling. However, defects in the fabrication are most likely to dominate in both cases and so a standard relative dielectric constant of 10 is justified.

A LEKID pixel geometry was decided upon and is shown in figure 5.8. This geometry is made up from the meander and capacitor components simulated earlier which we should be able to tune later for matching optically to free space.
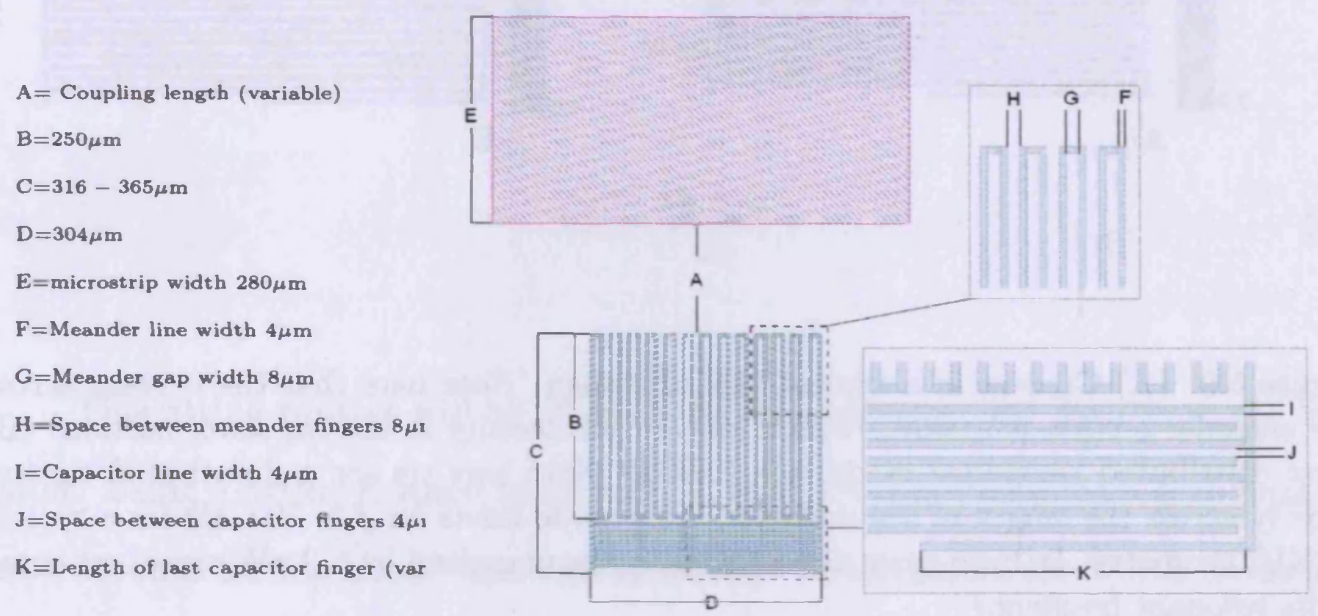

Figure 5.8: Schematic of the standard LEKID design

The design shown in figure 5.8 was drawn into a Sonnet layout. The meander was simulated using square turns to reduce simulation time. To measure the mutual inductance of the resonator we needed to know the resistance of the LEKID element. This value can be entered directly into Sonnet in $\Omega /$ square. The resistance of the LEKID as a lumped element resonator can be found by considering the areas where current flows in the circuit. If the LEKID is resonating as a 'true' 
lumped element resonator the current distribution across the meander should be uniform and be reduced in each interdigital finger of the capacitive section. To check this is indeed the case we can view the current in the LEKID using Sonnet's 'emvu' tool.

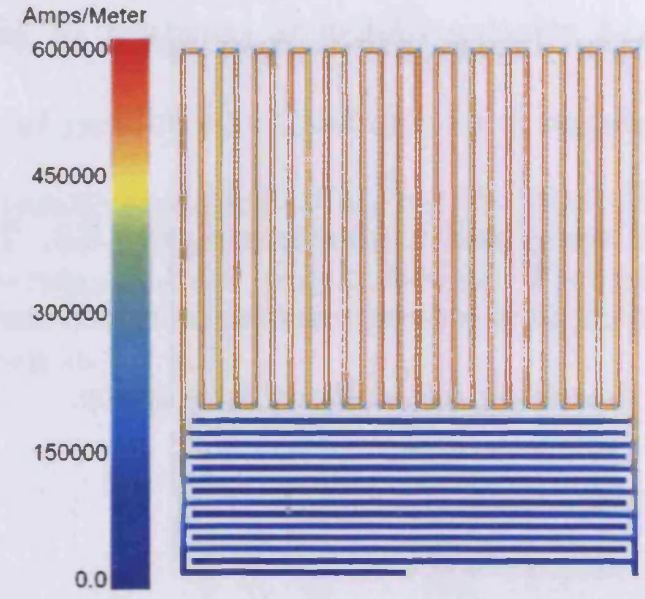

A

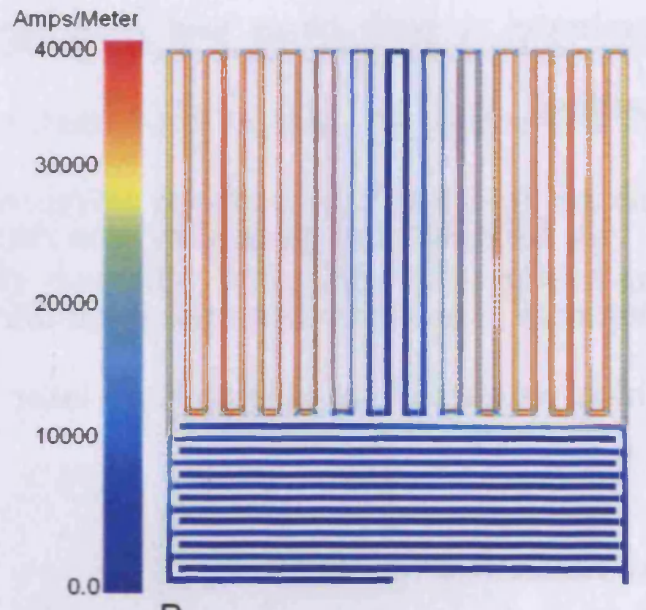

B

Figure 5.9: (A) Current in a typical LEKID design. Note here that the current across the meander section is uniform with a low current density in the capacitor section. (B) First distributed resonance mode in a LEKID. Note here we see a distributed current density across the length of the meander. This mode exists for $f>30 \mathrm{GHz}$ for a typical LEKID geometry. In both cases the LEKID has been excited by a $2 \mathrm{~mW}$ signal set equal to its resonant frequency

Fig 5.9 confirms that the LEKID is resonating in a lumped element mode as we observe a uniform current distribution. The total resistance for our LEKID model $(R)$ can be found by calculating the number of squares in the meander and then multiplying this by the $\Omega$ /square value we entered into the simulation. Each meander consists of $\approx 1600$ squares which contribute to $\mathrm{R}$ in our lumped model. We used a $\Omega$ /square value of $0.5 \times 10^{-6}$ for our simulations and designed the microstrip feed-line to be $50 \Omega$. We can now use equation 4.53 to calculate a 
value for $M$.

$M$ was simulated for a range of coupling distances ranging from $150 \mu \mathrm{m}$ to $450 \mu m$ from the top of the meander to the feedline. In each case, $Q_{u}$ was measured by fitting the S21 data to a modified version of equation 4.43 where we have written the resonator impedance in terms of $Q, \Delta \omega / \omega_{0}$ and $S 21_{\min }$ as in equations 4.10 and 4.53;

$$
\begin{gathered}
S 21=S 21_{\min } \frac{1+2 j Q x}{1+S 21_{\min } 2 j Q x}, \\
\text { where } \\
x=\frac{\omega_{0}-\omega}{\omega_{0}} .
\end{gathered}
$$

This fitting routine will be of great use for analysing real data from the VNA later. Using a fitting method to extract $Q_{u}$ from the simulated resonance data will also account for the small variations in current we see across the width of the strip which are not accounted for by simply counting the resistive squares . The fitting routine was crossed referenced the value of $Q_{u}$ from the analytical calculation of $Q_{u}\left(Q_{u}=\omega_{0} L / R\right)$ to verify that the fit was working properly.

The plots in figures 5.10 and 5.12 also allow us to calculate a loaded quality factor $\left(Q_{L}\right)$ for the LEKID. We decided to measure $Q_{L}$ using three different methods to compare results. This was an important exercise as $Q_{L}$ will determine the response of a LEKID to a change in inductance. The first method was a simple 


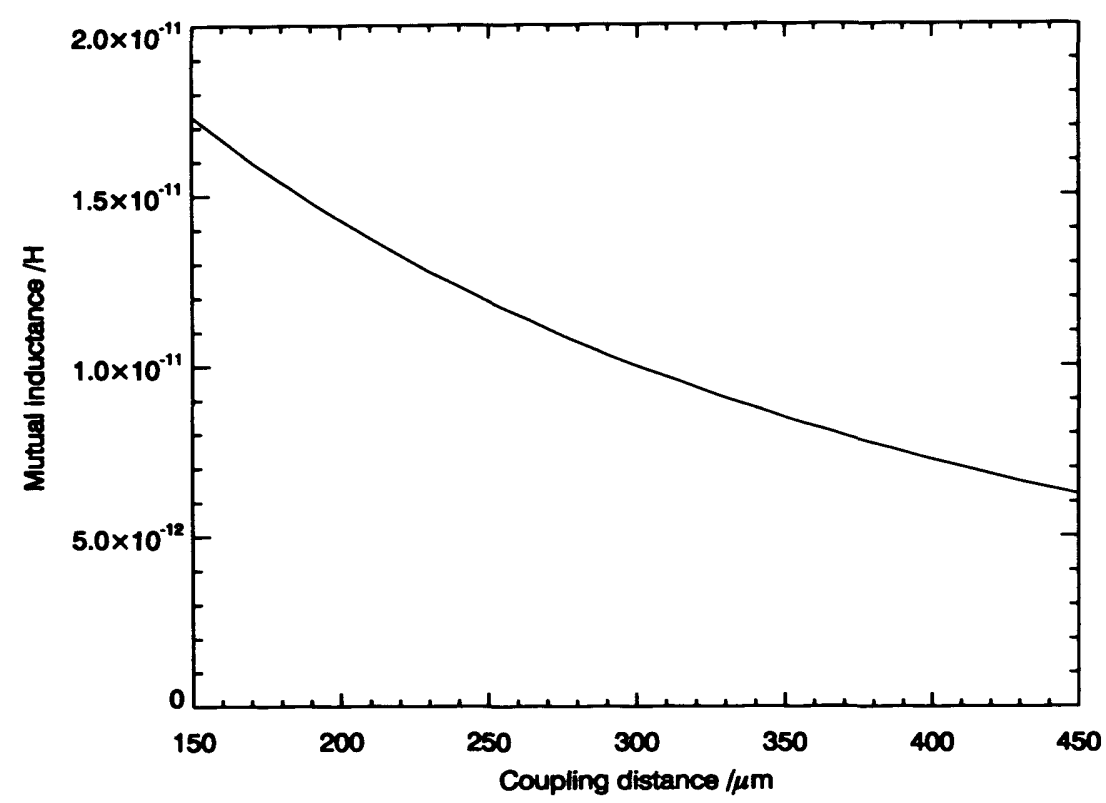

Figure 5.10: Plot of simulated mutual inductance vs coupling distance for a typical LEKID device.

measurement of the resonant frequency divided by the $3 \mathrm{~dB}$ bandwidth. The second method used equation 4.50 taking the calculated $g$ from equation 4.53 and the $Q_{u}$ from the fit. The third method takes the bandwidth at the point half way between the carrier level off resonance and $S 21_{\min }$ i.e. where $\mathrm{S} 21=$ carrier-(carrier$S 21_{\min }$ )/2. (Note that for simulations of a lossless matched system, the carrier level will be equal to one in magnitude). 


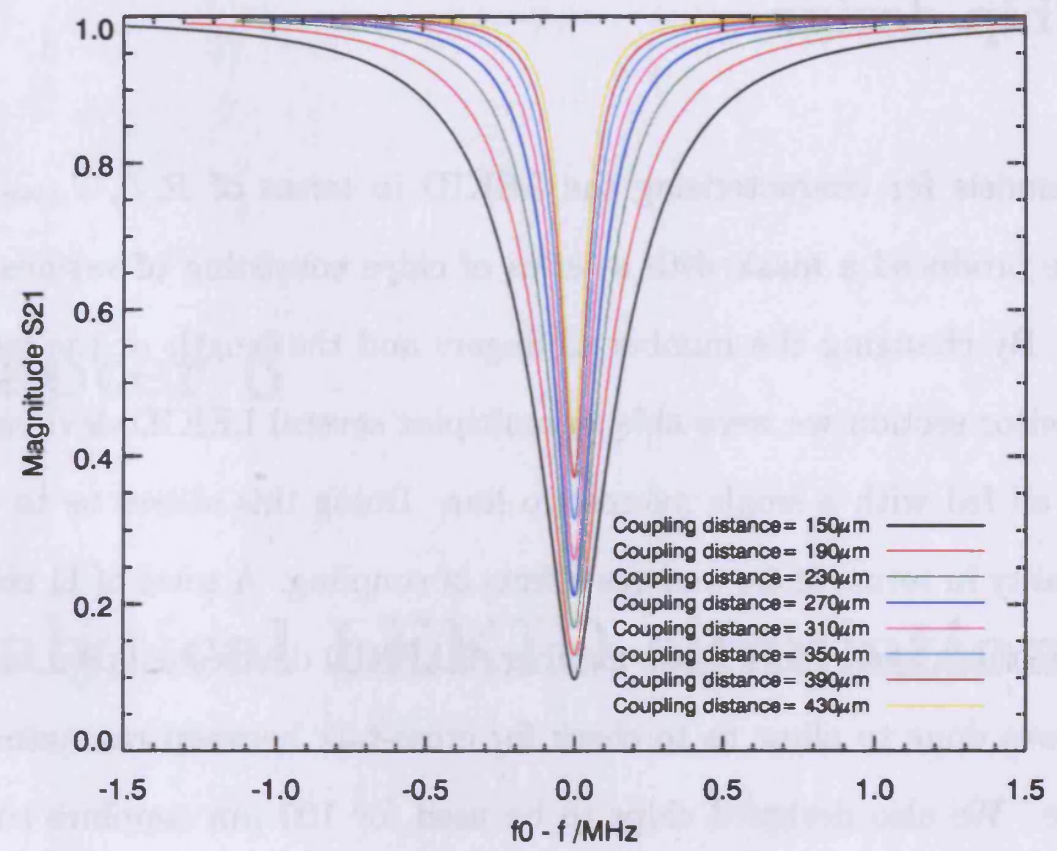

Figure 5.11: Plot of simulated S21 curves for various coupling distances. Here a 1600 square meander has been simulated with $5 \times 10^{-7} \Omega$ /square sheet resistance

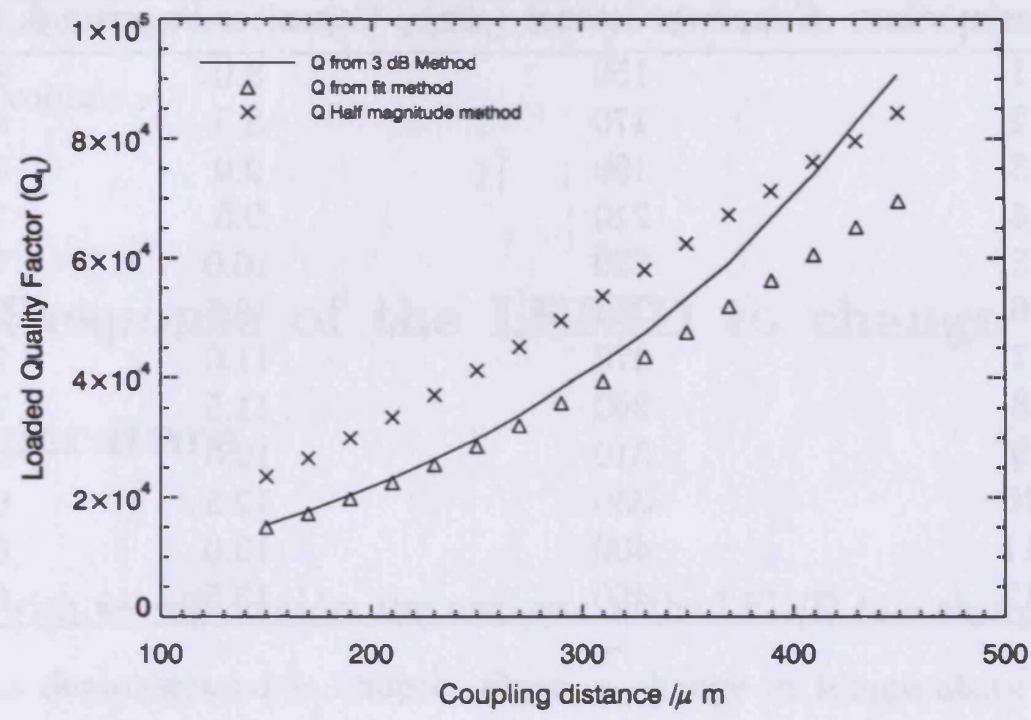

Figure 5.12: Plot of simulated $Q_{L}$ vs coupling distance, $Q_{u}=150000$. This plot shows good agreement between the three methods used for a well coupled resonator but note the $3 \mathrm{~dB}$ method starts to diverge under weaker coupling. 


\subsection{Chip design}

With the models for characterising the LEKID in terms of $R, L, C, \omega_{0}$ and $M$ in place, we produced a mask with a series of chips consisting of various LEKID resonators. By changing the number of fingers and the length of the last finger of the capacitor section we were able to multiplex several LEKID devices on to a single chip all fed with a single microstrip line. Doing this allows us to measure the film quality in terms of $Q_{u}$ and the effects of coupling. A total of 11 chips were added to the mask each varying the number of LEKID devices coupled to a single line. This was done to allow us to check for cross-talk between resonators if this should arise. We also designed chips to be used for $100 \mu \mathrm{m}$ sapphire substrates which consisted of a narrower $(90 \mu \mathrm{m})$ feed-line. The chip most used in testing was chip 3 , the details of which are shown in table 5.2 .

Table 5.2: Chip 3 design details

\begin{tabular}{cccc}
\hline \hline Resonator number & Coupling distance $(\mu \mathrm{m})$ & Capacitor fingers & $f_{0}(\mathrm{GHz})$ \\
\hline 1 & 150 & 8.0 & 8.47 \\
2 & 170 & 8.5 & 8.24 \\
3 & 190 & 9.0 & 8.02 \\
4 & 210 & 9.5 & 7.81 \\
5 & 230 & 10.0 & 7.62 \\
6 & 250 & 10.5 & 7.44 \\
7 & 270 & 11.0 & 7.28 \\
8 & 290 & 11.5 & 7.12 \\
9 & 310 & 12.0 & 6.97 \\
10 & 350 & 12.5 & 6.84 \\
11 & 400 & 13.0 & 6.71 \\
12 & 450 & 13.5 & 6.58 \\
\hline
\end{tabular}




\section{Chapter 6}

\section{Analytical LEKID simulations}

The details covered in chapters 3,4 and 5 puts us in a position to be able to model the response of a LEKID resonator to a change in pair / quasi-particle density. Here we will demonstrate that the response of the LEKID will depend on operating temperature, loaded quality factor, internal to total inductance ratios and film volume.

\subsection{Response of the LEKID to change in tem- perature}

To start with we will consider the response of the LEKID to a change in temperature. As demonstrated in chapter three, a change in temperature will cause a change in total internal inductance and resistance of an isolated superconducting strip. Here we consider the LEKID meander as being isolated, that is to say that the presence of the ground-plane does not affect the current density in the mean- 
der. In reality the ground-plane will have some influence on the current density in the meander but this effect should be small due to the fact that the groundplane is far from the meander compared to the meander line width. Hence we can treat each line in the meander as simply being an isolated strip and apply the equations for internal inductance derived in chapter three. The devices designed in chapter 5 consisted of a meander of 26 lines each $4 \mu \mathrm{m} \times 250 \mu \mathrm{m}$ giving a total of approximately 1600 squares. We measured the external inductance of the meander from the Sonnet simulations in chapter 5 to be approximately $2.5 \mathrm{nH}$. We can therefore simulate the response of this device with a given mutual inductance $(\mathrm{M})$ to a change in temperature by calculating the change in total inductance $\left(L_{\text {ext }}+L_{\text {int }}\right)$ with change in temperature. Figure 6.1 shows the response of a $100 \mathrm{~nm}$ thick LEKID with a mutual inductance of $2 \times 10^{-11} \mathrm{H}$ and a resonant frequency of $7 \mathrm{GHz}$ at $250 \mathrm{mK}$ warmed to $300 \mathrm{mK}$.

These are relatively high temperatures for an aluminium LEKID hence we see a large change in $Q$ factor along with a large shift in resonant frequency. The phase response of the same LEKID can be seen in figure 6.2

As will be discussed later, the response of KID devices is read out with an IQ mixer. An IQ mixer effectively measures the real and imaginary parts of S21. There are two outputs to an IQ mixer which represent the mixed in-phase (I) and out-of-phase (Q) parts of the input signals to the RF and LO ports of the mixer. Figure 6.3 shows the real and imaginary parts of S21 plotted against each other to simulate the normalised response of the IQ mixer outputs.

Here we see the resonant feature shrink as we move to higher temperatures. This effect is caused by the increase in transmission on resonance due to the decrease in the coupling coefficient $(g)$ as the $Q$ reduces. 


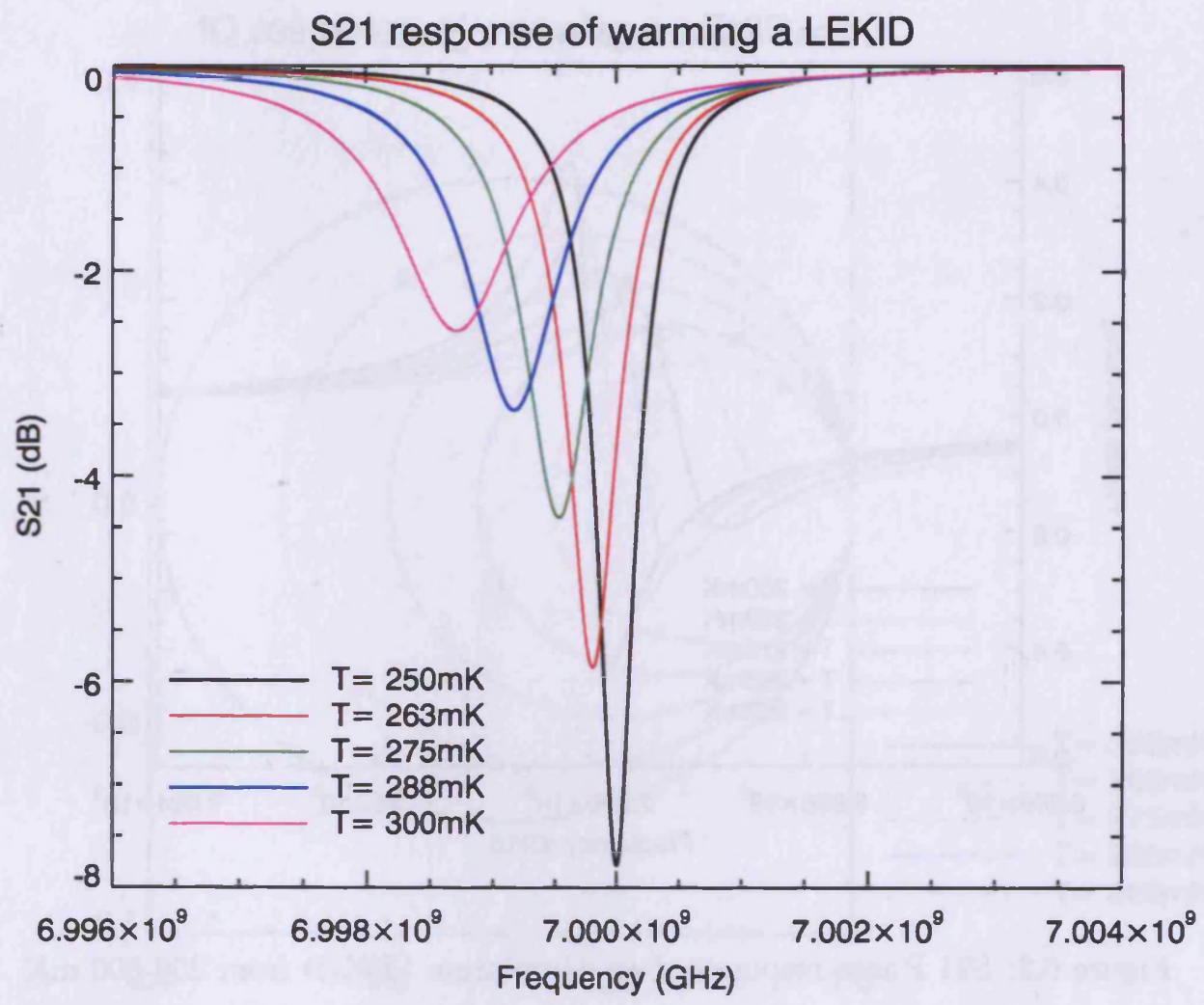

Figure 6.1: S21 Response of an aluminium LEKID from $250-300 \mathrm{mK}$ using data from figure 3.11 and 3.12

From the plots in figures 6.1 and 6.2 we can see a clear demonstration of the change in S21 in both amplitude and phase for a fixed frequency of $7 \mathrm{GHz}$ is dependent on the shift in resonant frequency. The shift in resonance will depend on the change in the complex impedance of the film as well as the internal to total inductance ratio. The ratio of internal to total inductance is usually defined as $\alpha$ and is given by $\alpha=L_{\text {int }} / L_{\text {tot }}$ where $L_{\text {tot }}=L_{\text {int }}+L_{\text {ext }}$. Writing the resonant frequency in the standard way we can write an expression for the change in resonant frequency with change in total inductance: 


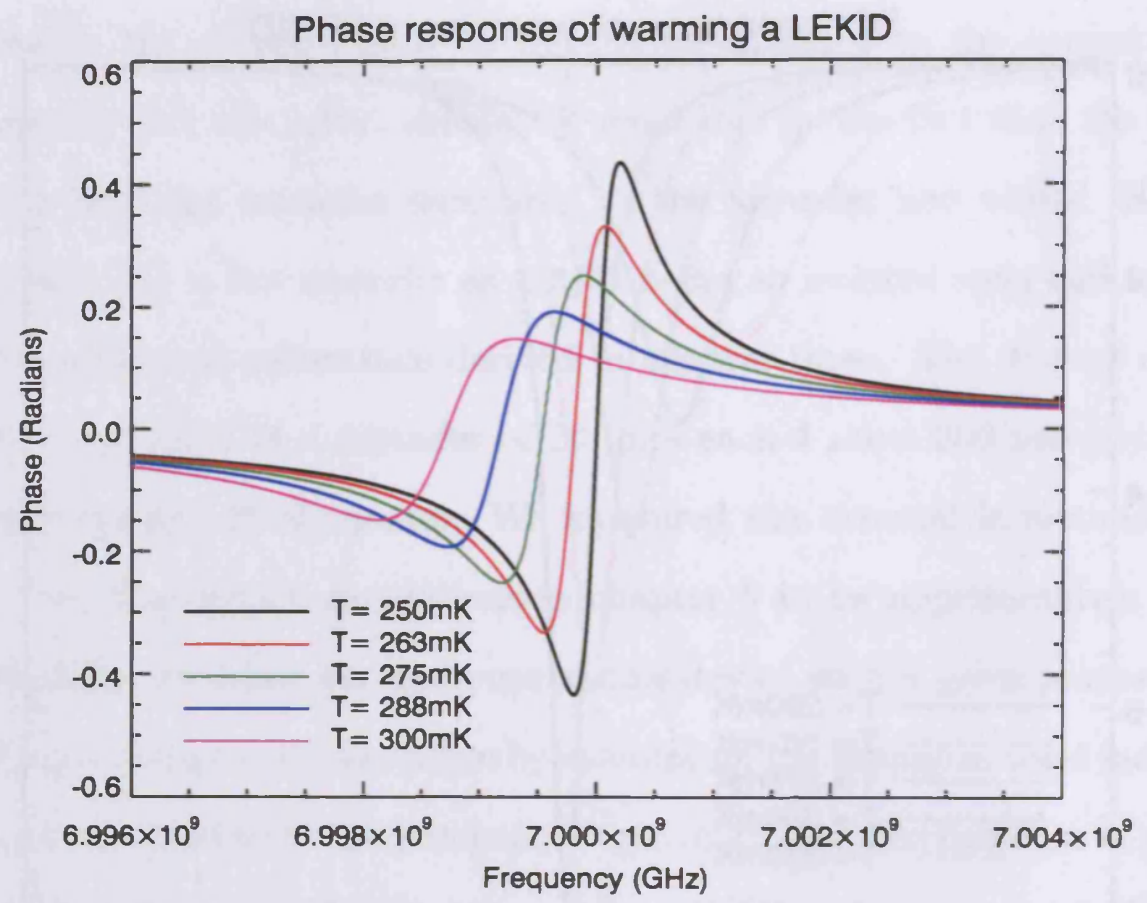

Figure 6.2: S21 Phase response of an Aluminium LEKID from 250-300 mK

$$
\begin{gathered}
\omega_{0}=\frac{1}{\sqrt{L_{t o t} C}}=L_{t o t}^{-\frac{1}{2}} C^{-\frac{1}{2}} \\
C^{-\frac{1}{2}}=\frac{1}{L_{t o t}^{-\frac{1}{2}} \omega_{0}} \\
\frac{d \omega_{0}}{d L_{t o t}}=-\frac{L_{t o t}^{-\frac{3}{2}}}{2} L_{t o t}^{\frac{1}{2}} \omega_{0}=\frac{-L_{t o t}}{2} \omega_{0} .
\end{gathered}
$$

Writing $L_{\text {tot }}$ in terms of $\alpha$ and $L_{\text {int }}$ gives

$$
\frac{d \omega_{0}}{d L_{t o t}}=-\frac{\omega_{0} \alpha}{2 L_{i n t}} \rightarrow d \omega_{0}=-\frac{\omega_{0} \alpha}{2 L_{i n t}} d L_{i n t}
$$




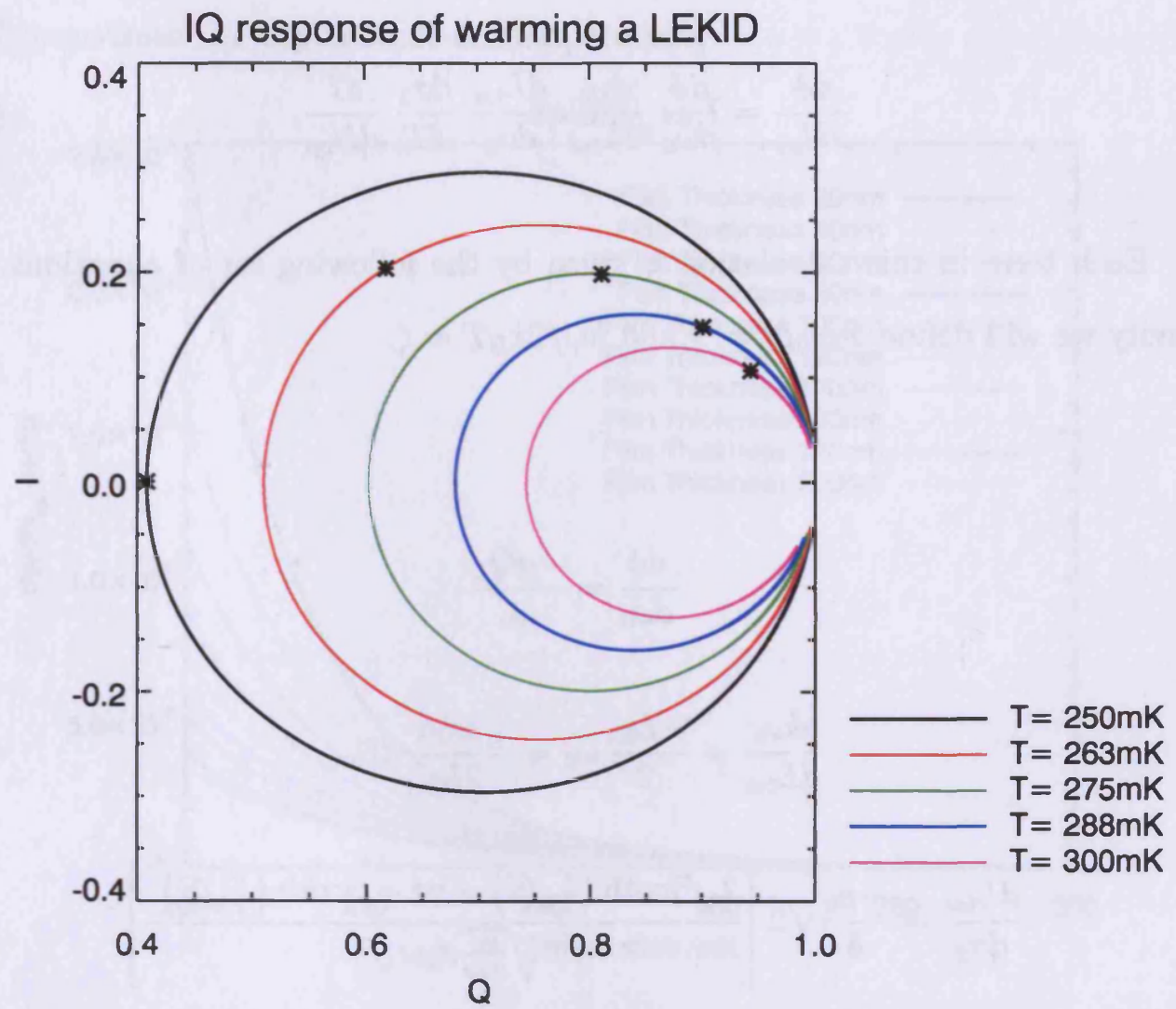

Figure 6.3: Imaginary S21 (Q) vs Real S21 (I) for an Aluminium LEKID warmed from $250 \mathrm{mK}$ to $300 \mathrm{mK}$. The plots form circles of increasing frequency in the clockwise direction. The points marked "* "represent the frequency (in this case $7 \mathrm{GHz}$ ) of a fixed microwave tone set to the resonant frequency of the lowest temperature resonator.

\subsection{LEKID response to change in quasi-particle density}

To determine the response of a LEKID to photon absorption we must consider the effect of breaking a single pair within the detector volume. The LEKID, like the distributed KID, is most responsive to changes in phase of S21 with change in resonant frequency so we need to calculate the change in phase with a change in quasi-particle density. This is done by the following calculation: 


$$
\frac{d \phi}{d N_{q p}}=\frac{d \phi}{d \omega_{0}} \frac{d \omega_{0}}{d L_{t o t}} \frac{d L_{t o t}}{d \sigma_{2}} \frac{d \sigma_{2}}{d T} \frac{d T}{d N_{q p}}
$$

Each term in this calculation is given by the following set of equations. For brevity we will define $\sigma_{2} \omega_{0} \mu_{0}=\gamma$ and $\hbar \omega / 2 k_{B} T=\zeta$.

$$
\begin{gathered}
\frac{d \phi}{d \omega_{0}}=\frac{-g Q_{L}}{\omega_{0}} \\
\frac{d \omega_{0}}{d L_{t o t}}=\frac{-L_{t o t}}{2} \omega_{0}=-\frac{\omega_{0} \alpha}{2 L_{i n t}} d L_{i n t} \\
\frac{d L_{t o t}}{d \sigma_{2}}=\frac{-\mu_{0}}{8} \sqrt{2}\left[\frac{2 \sqrt{\gamma} \operatorname{coth}\left(\frac{t}{2} \sqrt{\gamma}\right)-\gamma t+\gamma \operatorname{coth}\left(\frac{t}{2} \sqrt{\gamma}\right)^{2}}{\sqrt{\frac{\mu_{0}}{\sigma_{2}}} \sigma_{2} \omega \sqrt{\gamma}}\right] \\
\frac{-\pi \Delta(T) \sigma_{n}}{\hbar \omega_{0} k_{B} T^{2}} \exp \left(\frac{2 \Delta(0)+\hbar \omega_{0}}{-2 k_{B} T}\right)\left[2 \Delta(0) I_{0}(\zeta)+\hbar \omega I_{0}(\zeta)-\hbar \omega_{0} I_{1}(\zeta)\right] \\
\frac{d T}{d N_{q p}}=\left[N 0 \frac{\sqrt{2 \pi}}{T} \Delta(0) \frac{k_{B} T+2 \Delta(0)}{\sqrt{k_{B} T \Delta(0)}} \exp \left(\frac{-\Delta(0)}{k_{B} T}\right) \operatorname{vol}\right]^{-1}
\end{gathered}
$$

Here, $t$ is the film thickness, $T$ is temperature, $I_{0}$ and $I_{1}$ are modified bessel functions of the first and second kind respectively and vol is the film volume in the meander section of the LEKID. This rather complicated set of equations gives us a full analytical expression for the LEKID responsivity taking into account every design aspect discussed for the LEKID and its variation with temperature through Mattis-Bardeen theory. At first glance it may seem difficult to understand how to maximise the response from these expressions. However it is clear that we want to maximise $g, Q_{L}$ and $\alpha$ while minimising vol. 6.3 has been plotted out in figure 6.4 
to demonstrate the dependance on temperature.

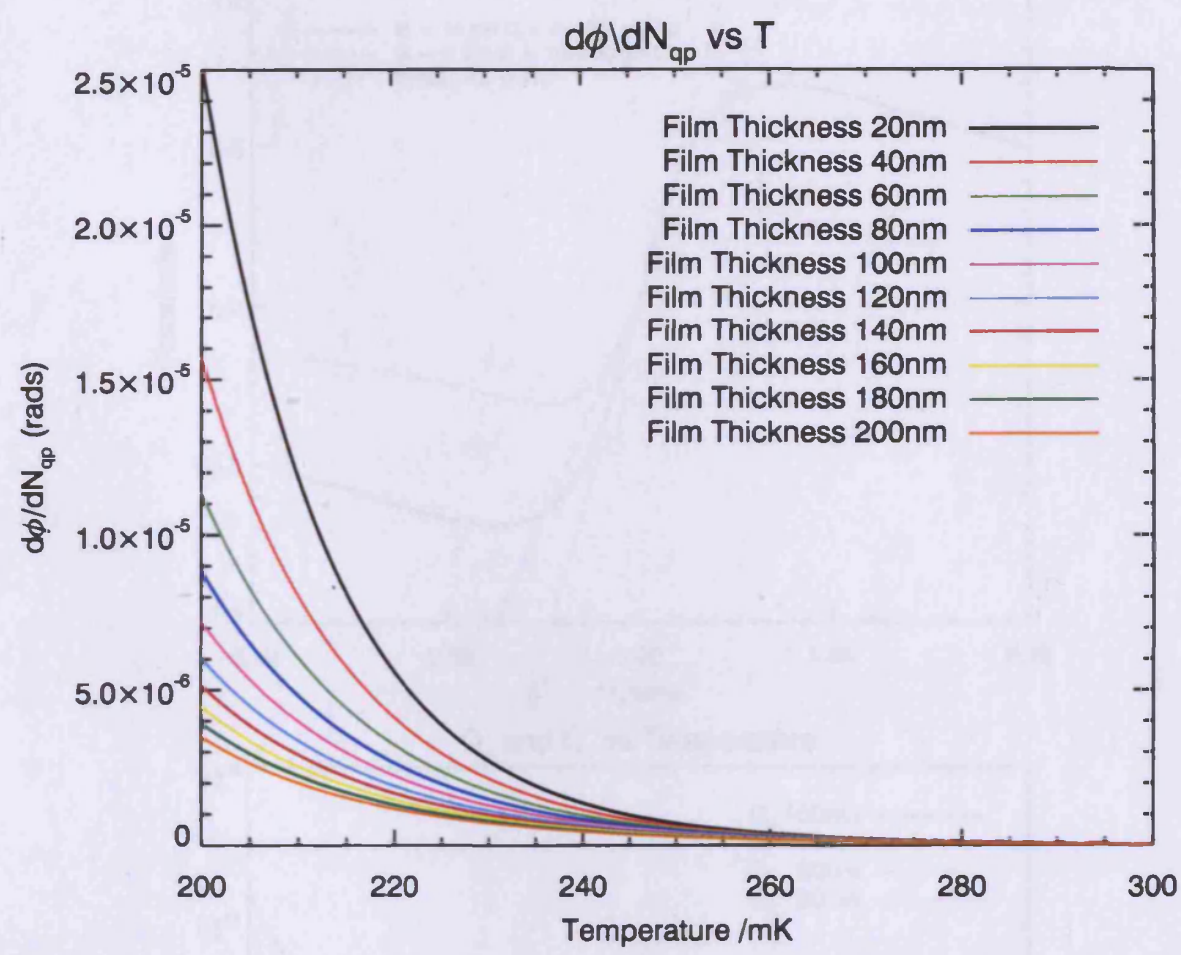

Figure 6.4: Phase responsivity per quasi-particle created for a $7 \mathrm{GHz}$ LEKID with a meander of 1600 squares, mutual inductance $M=10 \mathrm{nH}$, external inductance $L_{\text {ext }}=2.5 \mathrm{nH}$ and transition temperature $T_{c}=1.2 \mathrm{~K}$

Figure 6.4 shows the expected phase response of a LEKID with a mutual inductance of $M=1 \times 10^{-11} \mathrm{H}$. Here we see an increase in phase response as we move to lower temperatures and film thickness. The increase in phase response as we move to lower temperatures is caused by several factors. From a microwave standpoint the reduction in temperature increases $Q_{u}$ as the film losses reduce. This increases the coupling coefficient $g$ and loaded quality factor $Q_{L}$ and hence increases responsivity through equation 6.4. This is demonstrated in figure 6.5.

The change in total internal inductance reduces as we move to lower temperatures however this factor is outweighed by the fact that the effective change in temperature for a given film volume with change in quasi-particle number rises 
exponentially as temperature reduces. This is demonstrated in figure 6.7. 

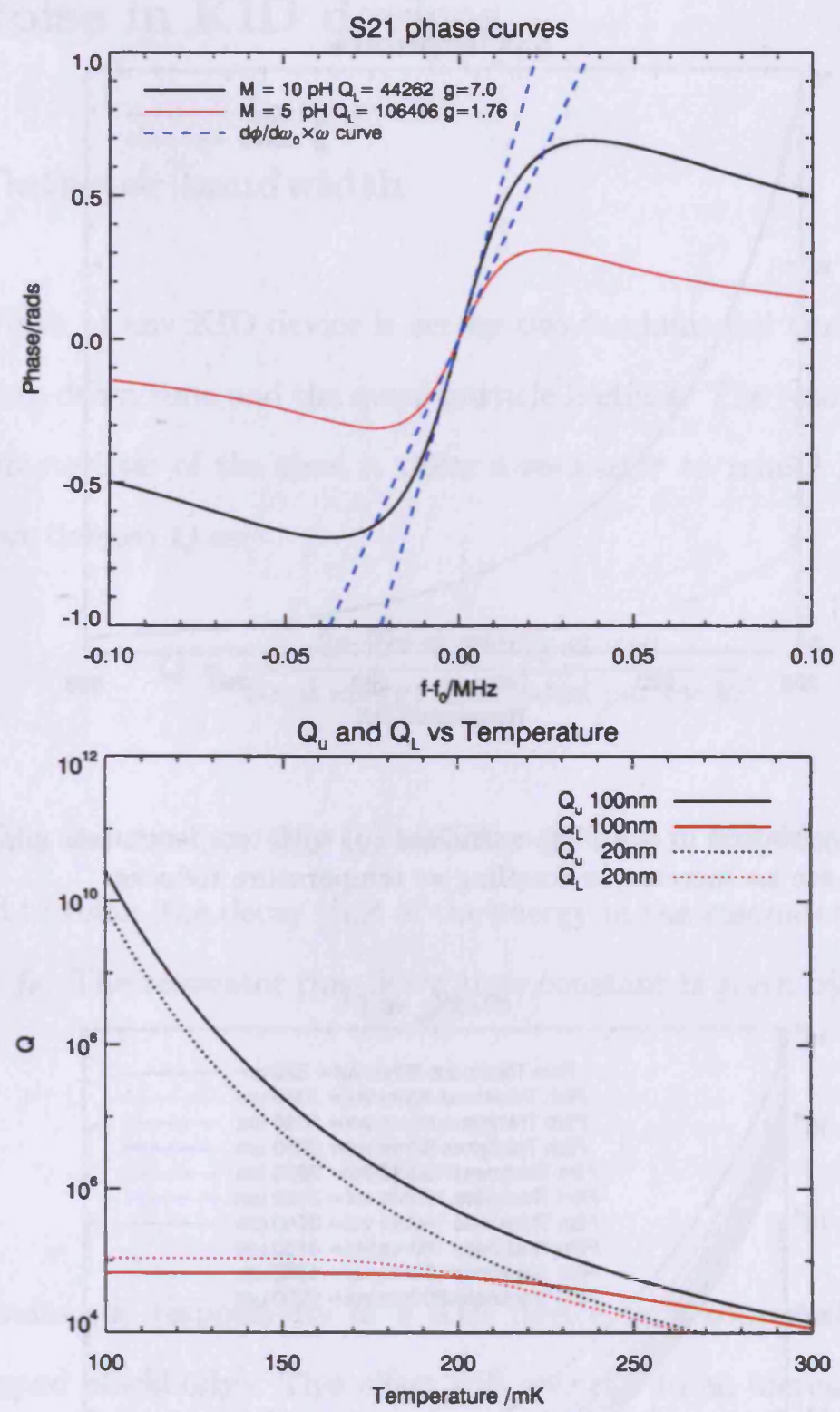

Figure 6.5: (Top) Phase variation about $f_{0}$ for two LEKIDs with an unloaded quality factor of 200000. The mutual inductance in each curve is varied by a factor of 2 and a dependance on $d \phi / d f$ is seen which is proportional to $Q_{L}$ and $g$ (Bottom) Variation of $Q_{u}$ and $Q_{L}$ with temperature for a fixed mutual inductance of $1 \times 10^{-11} \mathrm{H}$. Here we see an increase in both $Q_{u}$ and $Q_{L}$ with decreasing temperature until around $200 \mathrm{mK}$. At this temperature the device reaches its couple limited quality factor and $Q_{L}$ remains approximately constant . It is also worth noting that $Q_{u}$ is reduced with reducing film thickness. This is brought about by the increase in kinetic inductance for thinner films. The thinner film shows a higher $Q_{L}$ value due to the decrease in coupling with decrease in $Q_{u}$. 


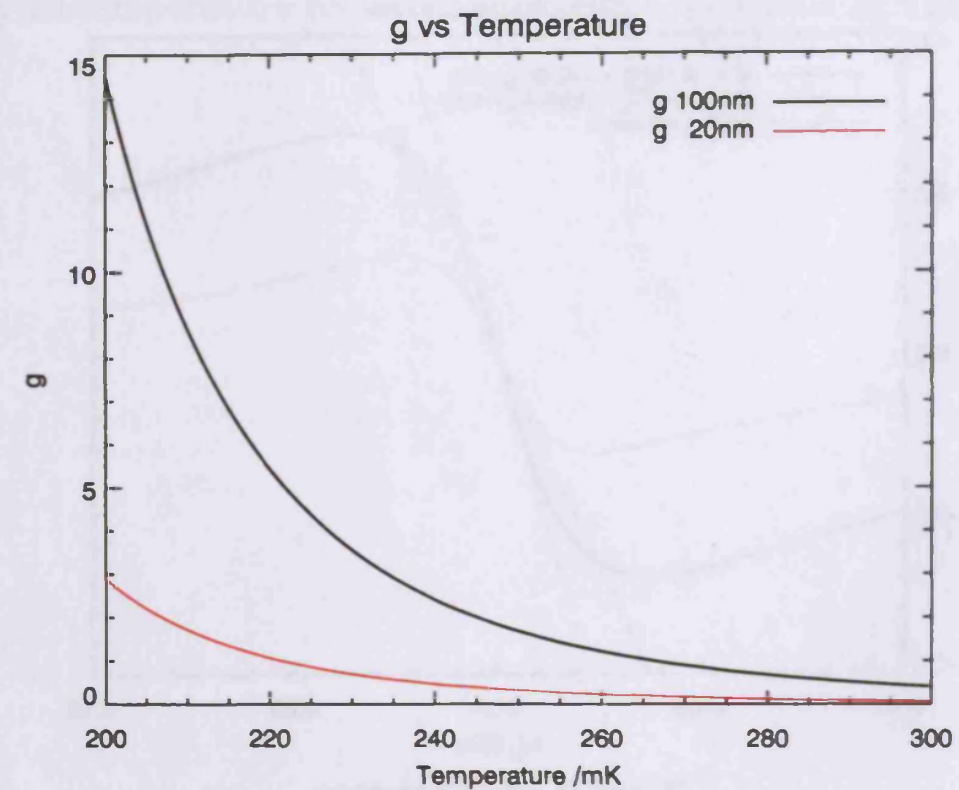

Figure 6.6: The variation in coupling coefficient $(g)$ with temperatures. $g$ is proportional to $Q_{u}$ hence we see an increase in coupling as temperature reduces.

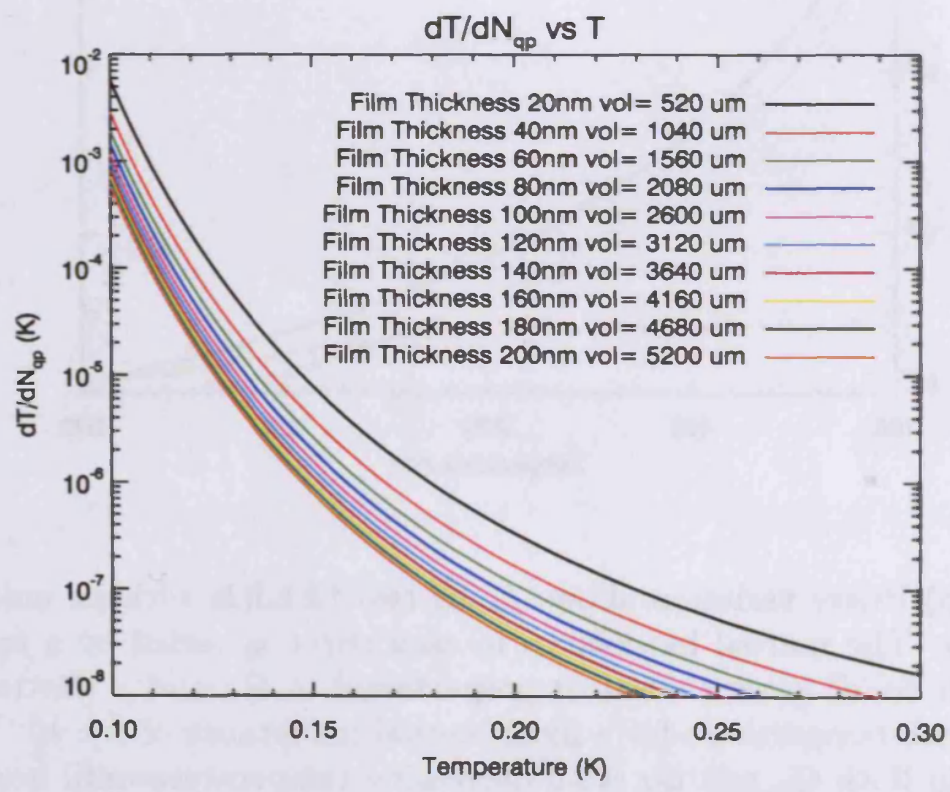

Figure 6.7: Effective change in temperature per quasi-particle broken for various LEKID film volumes. Here we see the greatest effective change in temperature in the lowest film volumes at the lowest temperatures. 


\subsection{Noise in KID devices}

\subsubsection{Detector bandwidth}

The bandwidth of any KID device is set by two fundamental time constants, the resonator ring-down time and the quasi-particle lifetime. The resonator ring-down time is characteristic of the time it takes a resonator to relax. For example, in Chapter 4 we defined $Q$ as:

$$
Q=\frac{2 \pi \text { Total energy stored }}{\text { Total energy dissipated per cycle }}
$$

Here $Q$ is the total $Q, Q_{t o t}$ (i.e includes $Q_{e}$ term). If a resonator is excited and allowed to relax, the decay time of the energy in the resonator is proportional to $Q_{\text {tot }}$ and $f_{0}$. The resonator ring-down time constant is given by [49]:

$$
\tau_{\text {res }}=\frac{Q_{\text {tot }}}{\pi f_{0}}
$$

This limits the responsivity of a KID device to a source of varying power (e.g. a chopped blackbody). This effect will give rise to an increased noise equivalent power (NEP) of a KID device as the frequency of variation in optical power approaches $1 /$ the ring-down time.

Like the ring-down time, the quasi-particle lifetime will also limit the sensitivity of a KID device to varying optical power due to the same effect. In most practical devices, the quasi-particle lifetime will dominate the time constant of the detector, as typical quasi-particle lifetimes for aluminium films are of the order of 
$100-500 \mu \mathrm{s}[3,50,51]$, whereas the ring-down time for a resonator with $Q_{t o t}=100000$ operating at $7 \mathrm{GHz}$ is $4.5 \mu \mathrm{s}$.

The variation of the optical power on a detector for most practical astronomy techniques from the $\mathrm{mm}$ to infrared is limited by the rate at which we can move a given optical component in the optics chain (for example the secondary mirror of a telescope or the scanning mirror of a fourier transform spectormeter). This limits the frequency of variation in power to typically less than $10 \mathrm{~Hz}$. This is far slower than either of the two time constants in a KID device and should therefore not pose any problems.

\subsubsection{GR noise}

The fundamental noise limit in any KID device is set by generation recombination noise (GR noise). GR noise is due to statistical fluctuations in the quasi-particle / pair density within any superconducting volume at $T>0$. These fluctuations are proportional to the number of quasi-particles within the film volume and the corresponding NEP is written as [5]:

$$
N E P_{g r}=2 \Delta \sqrt{N_{q p} / \tau_{q p}}
$$

The GR noise of a KID device can be minimised by reducing film volume and temperature. Both these actions lead to a reduced number of quasi-particles in the detector and hence lower GR noise. The effect of reduced temperature and film volume on GR noise for a superconductor with a fixed quasi-particle lifetime is illustrated in figure 6.8 


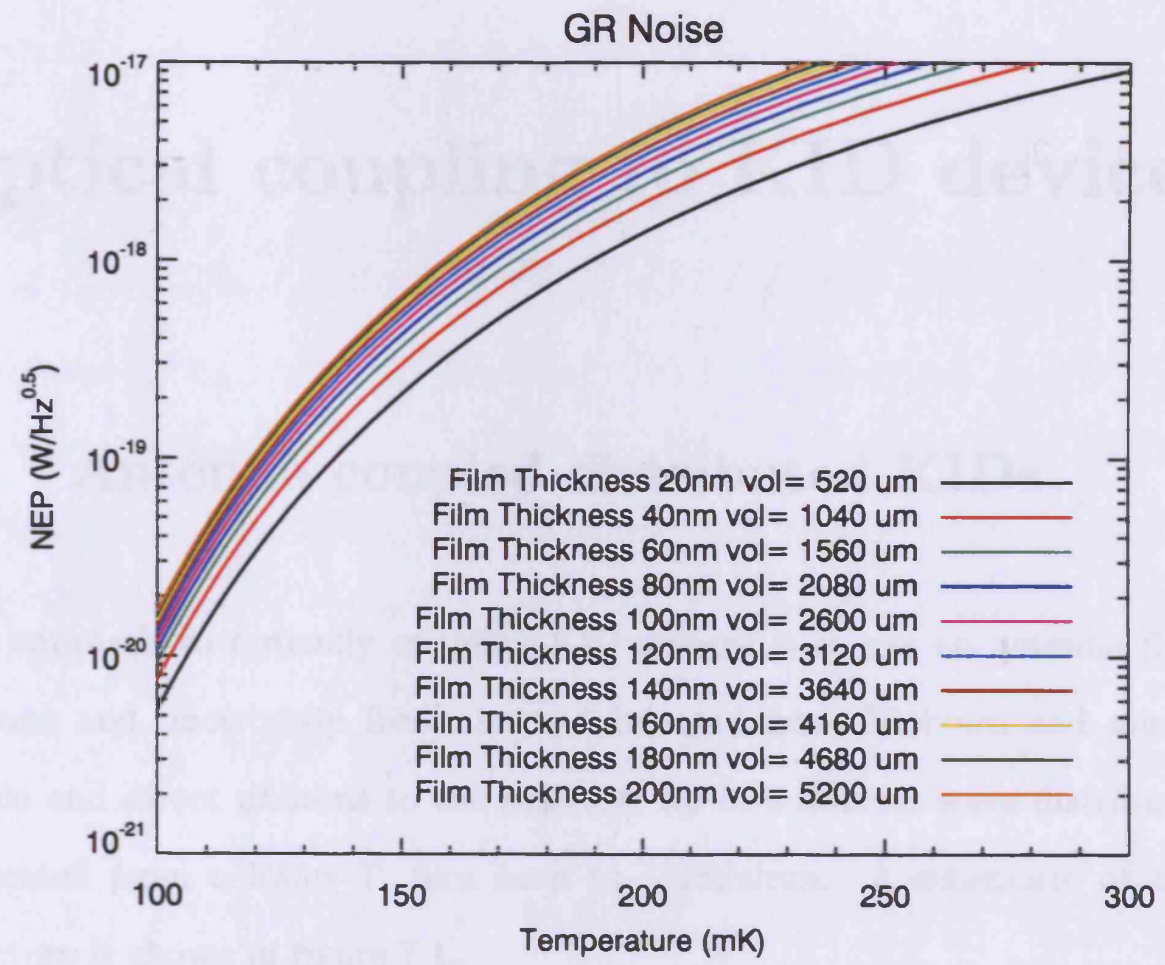

Figure 6.8: GR noise as a function of temperature for an aluminium LEKID with a quasi-particle life-time of $100 \mu s$ 


\section{Chapter 7}

\section{Optical coupling to KID devices}

\subsection{Antenna coupled distributed KIDs}

One approach to optically coupling KID devices is to use an antenna [3,4]. An antenna and mictrostrip feedline are fabricated from Niobium and are used to couple and direct photons to the sensitive tip of a quarter-wave distributed KID fabricated from a lower $T_{c}$ film such as aluminium. A schematic of the basic structure is shown in figure 7.1 .

This device can be used to couple to photons between the two band gap energies of the superconducting films (in this case $\Delta_{A l}<E<\Delta_{N b}$ ). Here a photon with energy $E<\Delta_{N b}$ can be coupled to by the antenna and propagate though the microstrip feedline with little loss. This can be ensured by working at $T<<T_{c}(N b)$ and fabricating thick film to minimise kinetic inductance. The microstrip feedline is run over the top of the aluminium KID separated by a deposited dielectric layer. The KID centre strip now acts as a lossy ground plane as the photons in the 


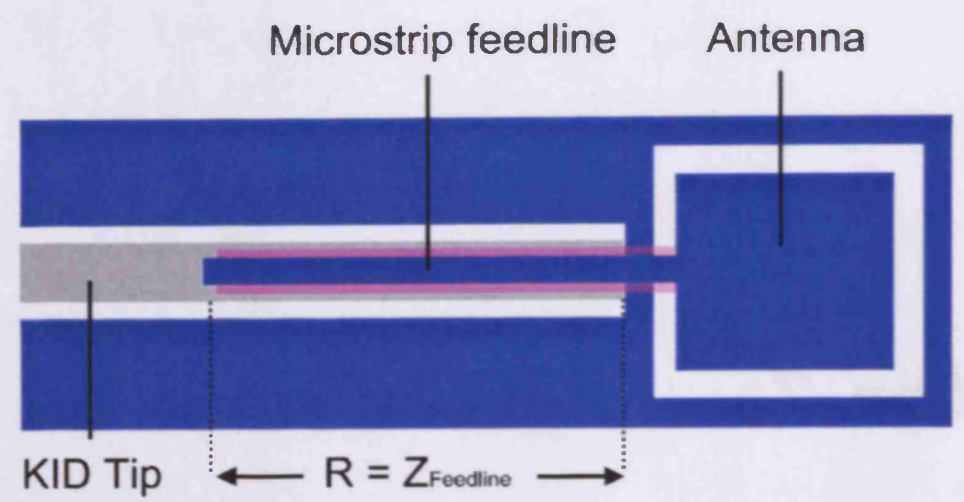

Niobium Dielectric Aluminium

Figure 7.1: Schematic of an antenna coupled distributed KID

feedline have an Energy $E>\Delta_{A l}$ and hence will break pairs in the sensitive tip of the KID. The feedline is terminated by connecting it to the KID at the point where the KID presents a matched normal impedance to ground. In practice this matching process is not too critical as the microstrip from the antenna is very lossy at these high frequencies and most of the signal is dissipated before reaching the termination point. Alternatively the microstrip feedline can be made long enough so that all the power is dissipated in the KID centre strip.

One advantage of this coupling method is on-chip filtering. By adding filter structures to the microstrip feed-line it is possible to define the observing band without the need for narrow band quasi-optical filters in the optics of the system. This makes multi-frequency detection possible in a single image plane of an instrument.

The gap energies of aluminium and niobium correspond to photon frequencies of $\approx 730 \mathrm{GHz}$ and $82 \mathrm{GHz}$ respectively. The cut-off at $730 \mathrm{GHz}$ makes this approach unsuitable for coupling to $\mathrm{THz}$ radiation. However this method has been shown 
to work at $\mathrm{mm}$ and submm wavelengths using a demonstration instrument at the Caltech Submm Observatory (CSO) [4]. Here successful images of Jupiter and the molecular cloud G34.3 were made at $240 \mathrm{GHz}$ and $350 \mathrm{GHz}$. The antenna in this case consisted of 16 slot antennas co-added in phase to a single microstrip feedline to form a phased array.

Antenna coupling does have some drawbacks, for example the optical efficiency of current phased array designs is around an order of magnitude lower than expected [4]. This is indicative of the complexity in designing phased arrays where lithographic tolerances are crucial to the overall performance. Another drawback is stray light. A distributed KID will be sensitive to photons directly absorbed in the ground-planes or centre strip of the device. This means that great care must be taken to shield the resonant element of the detector from coupling directly to the source and causing spurious signals.

\subsection{Quasi-particle trapping}

At higher frequencies such as optical, UV and X-ray, coupling has been achieved though quasi-particle trapping. Here quasi-particles are created in an absorbing patch of superconductor with a band gap energy higher than that of the KID. The tip of the KID is connected to the absorber directly and is used to trap quasiparticles generated upon photon absorption. This method works on the principle that quasi-particles generated in the absorber will diffuse into the sensitive tip of the KID. Here the higher energy quasi-particles will quickly scatter into the lower energy states from which they were forbidden to exist in while in the absorber. These scattering processes will create more quasi-particles through phonon emis- 
sion and will exist in energy states just above the gap energy of the KID. These quasi-particles are now trapped in the KID as until they condense into pairs they lack the energy to occupy any of the free states available in the absorber. This process is demonstrated in figure 7.2 .

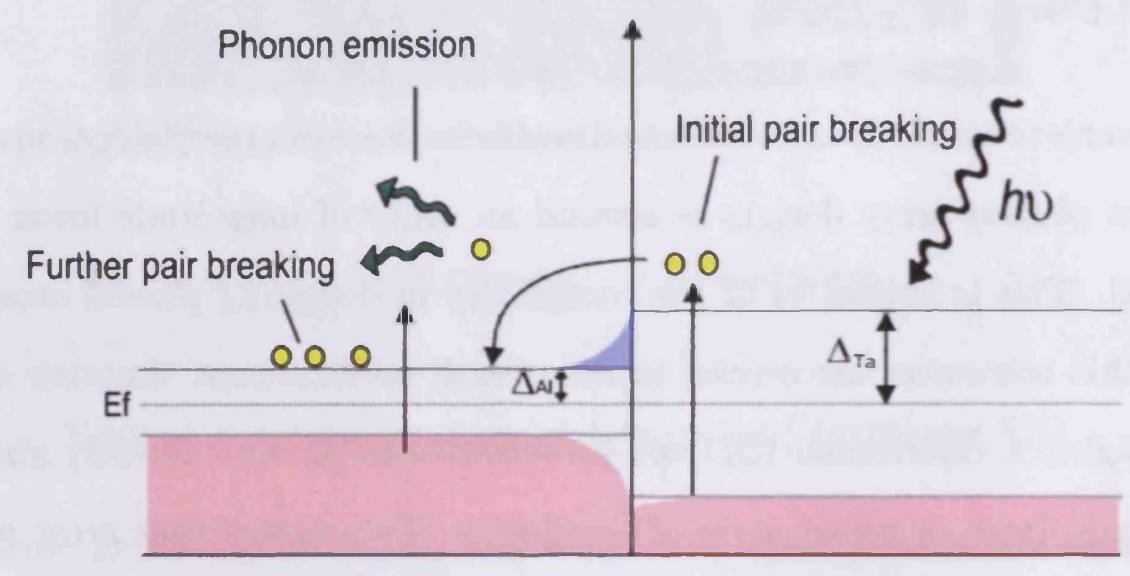

Aluminium

Tantalum

Figure 7.2: Schematic of a quasi-particle trap. Here aluminium has been used as the KID material and tantalum has been used as the absorber material. A photon with $h \nu>\Delta_{T a}$ creates quasi-particle excitations. These diffuse into the aluminium KID and lose energy through scattering creating phonons. These phonons create further quasiparticle excitations in the aluminium KID. The quasi-particles in the aluminium KID are trapped due to the lack of available states in the Tantalum absorber.

The quasi-particle trap relies on the quasi-particle lifetime being long. In this case the quasi-particles will survive long enough to random walk into the aluminium KID. The quasi-particle diffusion length can be written as [6] :

$$
l=\sqrt{D \tau_{q p}}
$$

where $D$ is the diffusion constant and $\tau_{q p}$ is the quasi-particle life-time. In clean tantalum films $D$ can range from $\approx 8-14 \mathrm{~cm}^{2} / \mathrm{s}$ for temperatures between 210 and $150 \mathrm{mK}$. Depending on the quasi-particle life-time in the film this can yield 
diffusion lengths $l$ as high as $\approx 250 \mu \mathrm{m}$ in relatively thick films $(200-300 \mathrm{~nm})[6,42]$.

For X-ray detectors used to measure photon energy, the absorption is due to the photon interacting with valance electrons in the material exciting them into the conduction band [52]. For the X-ray photon to be absorbed, thick films are required to increase the chances of interaction beween the photon and an electron. This requirement works in favour of the trapping method as the quasi-particle life-time and hence diffusion length shows a strong dependance on film thickness. However the absorption method for $\mathrm{THz}$ photons is different to that of higher energy photons where scattering events are responsible for photon absorption. For a $\mathrm{THz}$ photon we rely on the photon interacting electrically with the absorber. Here a photon will generate currents in the absorbing film which are responsible for pair breaking. In order for the absorber to absorb these photons and not simply reflect them we need to impedance match the absorber to free space. Free space has an impedance of $377 \Omega$ so in order to maximise absorption, the sheet resistance of the absorber need to be $377 \Omega$ per square. The normal state resistance of tantalum at room temperature is around $13 \times 10^{-8} \Omega \mathrm{m}$ [53]. In high quality film (needed for long diffusion lengths) tantalum has $R R R$ values of around 17-26 [6,42]. So in order to match a tantalum absorber to free-space a film thickness of less than $1 \mathrm{~nm}$ is required. Illuminating the absorber through a high dielectric constant substrate such as silicon $\left(\epsilon_{r} \approx 11-12\right)$ [53] means a lower sheet resistance is required $\left(Z_{S i}=377 / \sqrt{\epsilon_{r}}=107 \Omega\right)$. This approach only gains us around a factor of 3.5 in film thickness.

To absorb a THz photon the absorber's minimum dimensions will need to be at least of the order of a wavelength $(200 \mu \mathrm{m})$. This fact, together with the need for extremely thin films significantly reduces the number of quasi-particles generated in the absorber surviving long enough to diffuse into the KID. This leads to a 
dramatic reduction in optical efficiency and so this method has been disregarded as a suitable optical coupling method for $\mathrm{THz}$ photometry.

\subsection{Partially filled distributed absorbers}

Operating at $\mathrm{THz}$ frequencies the quasi-particle trap is an example of a distributed absorber. In a distributed absorber, photon absorption occurs over a distance comparable to a wavelength as opposed to the termination of an antenna where the power is deposited in a small volume of dimensions much smaller than a wavelength (lumped absorber). The LEKID meander is a distributed absorber with a reduced filling factor. This is akin to a bolometer with a mesh absorber [54]. Here a mesh is used instead of a solid absorber to increase the effective sheet impedance of the absorbing element. Now the impedance presented to an incoming photon is given by:

$$
Z_{\text {eff }}=\frac{Z}{F}=Z \frac{s}{w}
$$

Here $Z_{\text {eff }}$ is the effective sheet impedance of the web, $Z$ is the impedance of the web material in $\Omega \mathrm{s} /$ square and $F$ is the filling factor. The filling factor is found by calculating the ratio of line widths $(w)$ to the space between lines in the web $(s)$. In order for the web to look like a solid sheet to an incoming photon the space between lines need to be of order $\lambda / 20$ or less. 


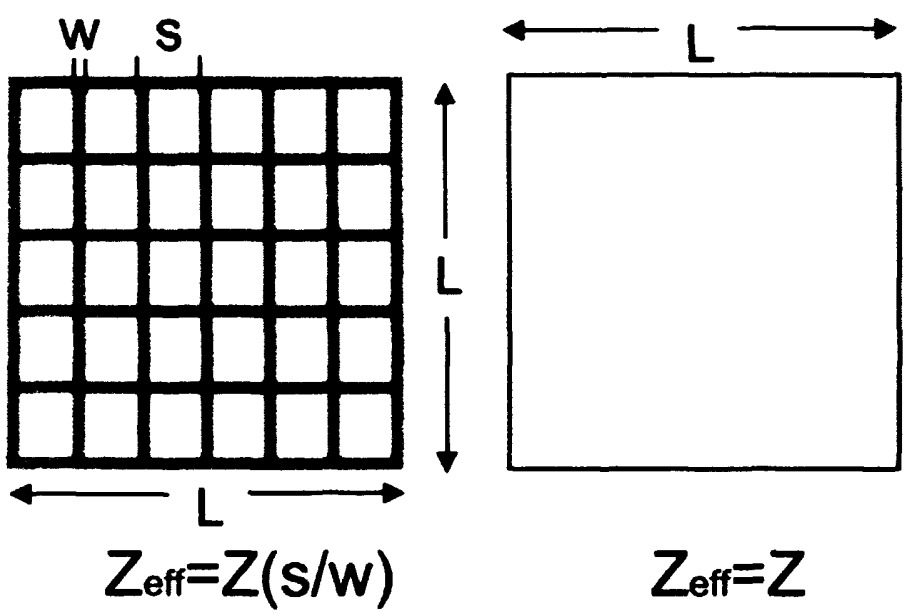

Figure 7.3: (Left) A schematic of a mesh absorber where the dimensions are exaggerated for demonstration. The effective sheet impedance is given by $Z_{\text {eff }}=Z(s / w)$ where $\mathrm{Z}$ is the normal sheet impedance of the material used in the web. The space between lines needs to be of order $\lambda / 20$ or less for the web to look like a solid sheet to an incoming photon (Right) A solid absorber. Here the sheet impedance is simply the sheet impedance of the material. In both cases the dimension $\mathrm{L}$ needs to be greater than $\lambda$.

\subsubsection{Impedance of a superconducting film at high fre-} quency

The meander section of the LEKID acts as the absorbing element to incoming photons. As shown in Chapter 3 the impedance of a superconducting film is a function of both frequency and temperature. At frequencies where $h \nu>2 \Delta$ the conductivity of a superconducting film reduces to its normal state conductivity $\sigma_{1}$. This is demonstrated in figure 7.4 , which shows the complex conductivity as a function of frequency calculated from the Mattis-Bardeen integrals for aluminium at a temperature of $100 \mathrm{mK}$. 


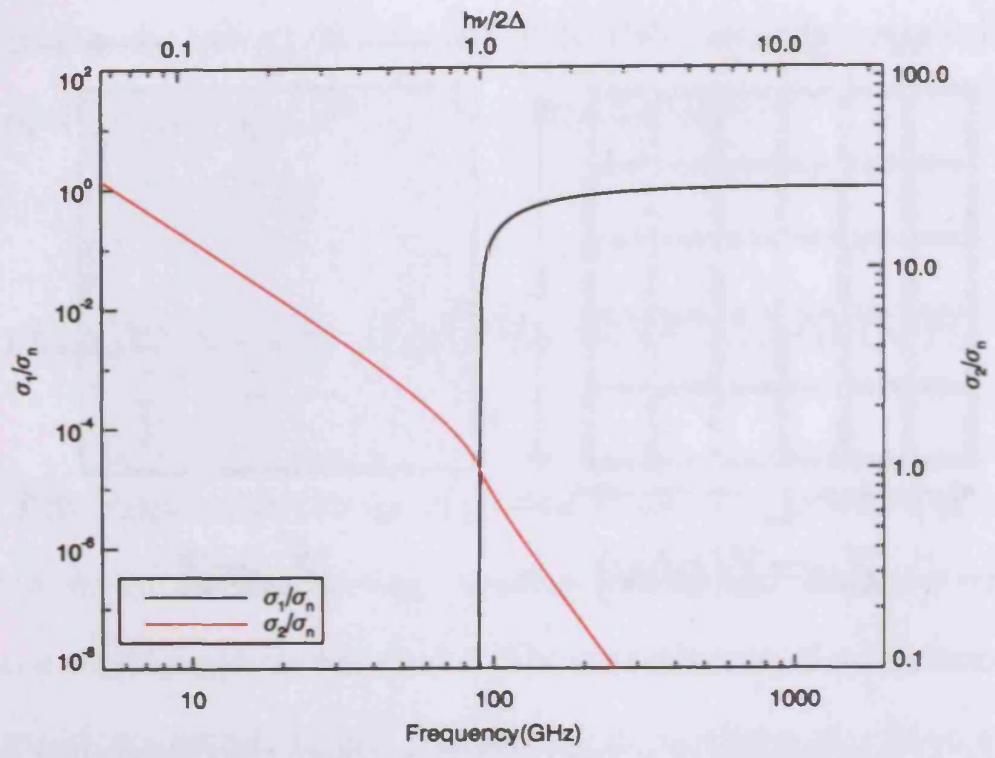

Figure 7.4: The complex conductivity Aluminium at $100 \mathrm{mK}$ as a function of frequency. Here we see at frequencies where $h \nu>2 \Delta \sigma_{2} \approx 0$ and $\sigma_{1} \approx \sigma_{n}$.

\subsubsection{Modelling absorption}

The LEKID meander acts as a single polarisation absorber. This is due to the meander lines looking like a set of parallel lines in one direction. Photons polarised in the direction of the meander can interact with it and break pairs. In the polarisation orthogonal to the meander lines the impedance is very large and an incident photon sees the meander as transparent. This idea is confirmed by a set of HFSS simulations. Here we model the meander as a set of infinitely long parallel lines in free space. The lines were made to give a matched impedance to free space following the argument of 7.2. Two simulations were run with the incident wave being polarised parallel and orthogonal to the meander lines. The plot in figure 7.5 demonstrates absorption being dominant for incident photons being polarised in the direction of the meander.

The maximum absorption for a resistive sheet in free-space is $50 \%$ and occurs 


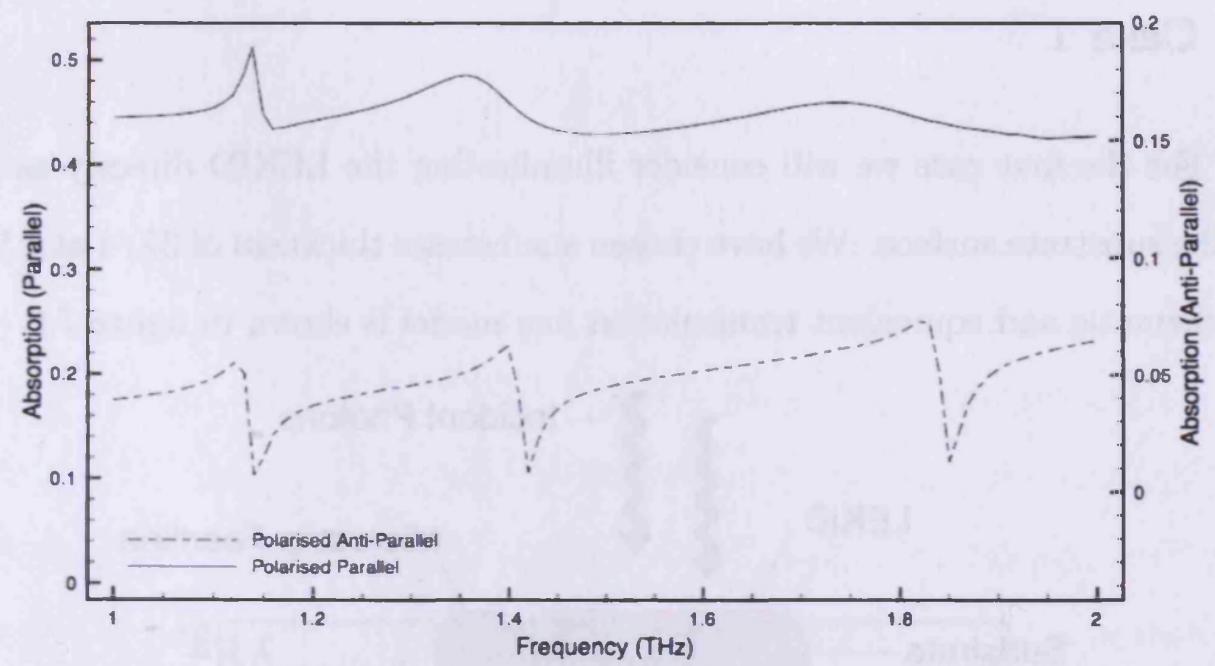

Figure 7.5: The polarised properties of the meander as an absorber. Here we see around $45 \%$ absorption for photons polarised parallel to the meander and less than $7 \%$ absorption for photons polarised orthogonal to the meander.

for a sheet impedance equal to that of free space. This can be improved upon with the addition of back-shorts, substrates and anti-reflection (AR) coatings. We modelled absorption under a set of optical arrangements using a transmission line model. Here we model each section of the detector as a transmission line with an impedance and length. The absorber is modelled as a shunt impedance to ground. The absorption is found by solving the effective ABCD matrix for the system and calculating S11 and S21. The absorption of the system is then given by:

$$
\text { absorption }=1-[S 11+S 21]^{2} \text {. }
$$

The ABCD matrix elements needed for each section of the model can be found in the appendix [40]. We consider here three options for optical coupling. 


\section{Case 1}

For the first case we will consider illuminating the LEKID directly as it lays on the substrate surface. We have chosen a substrate thickness of $3 \lambda / 4$ at $1.5 \mathrm{THz}$. A schematic and equivalent transmission line model is shown in figure 7.6.
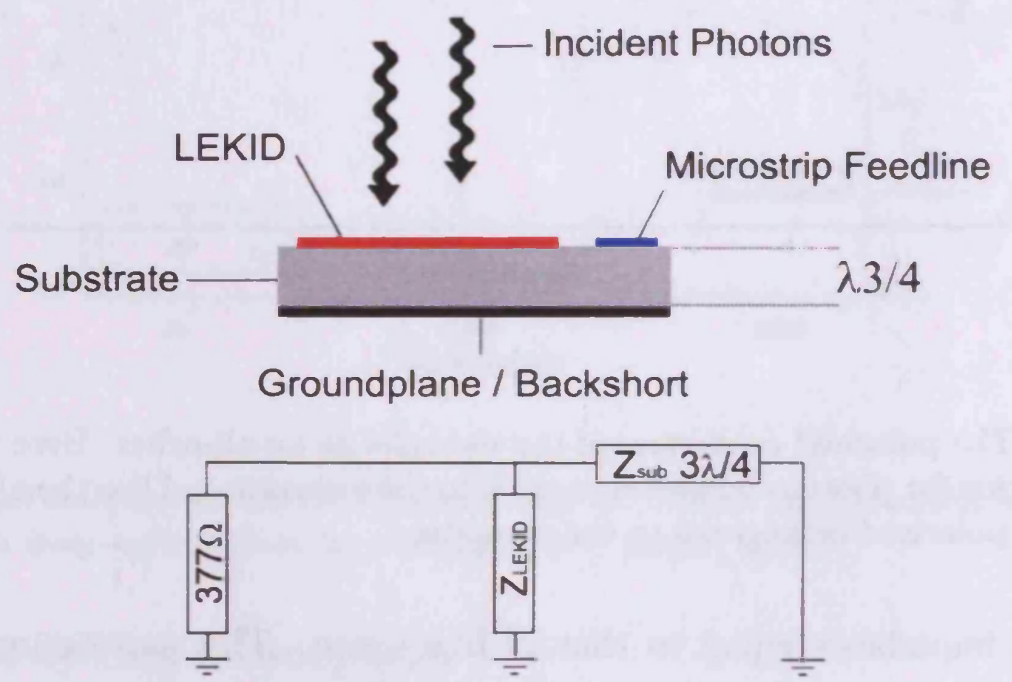

Figure 7.6: (top) Schematic of a LEKID under direct illumination. (bottom) Equivalent transmission line model. Here $Z_{\text {sub }}$ is the substrate impedance and $Z_{L E K I D}$ is the effective sheet impedance of the LEKID meander. 


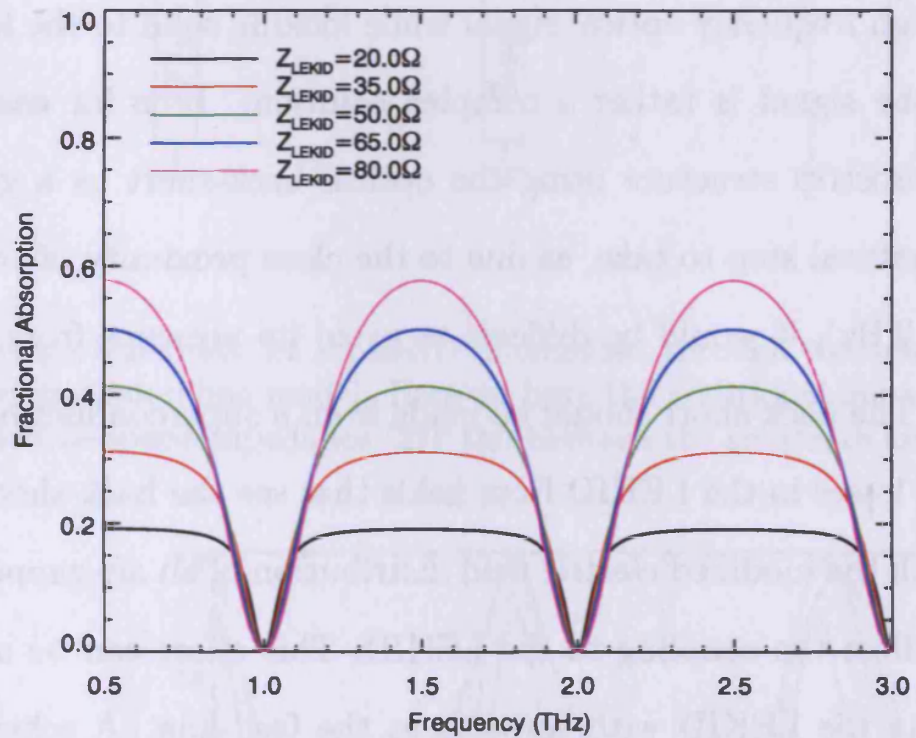

Figure 7.7: Absorption for a LEKID illuminated directly with radiation polarized parallel to the meander Here sapphire has been used as the substrate material $\left(\epsilon_{r} \approx 10\right)$. 


\section{Case 2}

The second case illuminates the LEKID through the substrate. In general this method works better as high permittivity substrates such as sapphire and silicon will have a far lower impedance than to that of free space. This means we can achieve good optical coupling using lower effective impedance films in the LEKID. To illuminate through the substrate we must remove the ground plane or replace it with a high pass filter. Replacing the ground plane with a mesh structure which will pass the high frequency optical signal while looking solid to the low frequency microwave probe signal is rather a complex solution. It is far easier to create an air gap microstrip structure using the optical back-short as a ground plane. This seems a natural step to take, as due to the close proximity of the back-short (50 $\mu \mathrm{m}$ at $1.5 \mathrm{THz}$ ), it would be difficult to avoid its presence from a microwave point of view. The back-short should be made from a superconducting material to avoid inducing losses in the LEKID from fields that see the back-short. The backshort along with the modified electric field distribution of an air-gapped microstrip feed-line will affect the coupling to the LEKID. This effect can be accounted for by repositioning the LEKID with respects to the feed-line. A schematic of this arrangement is shown in figure 7.8 

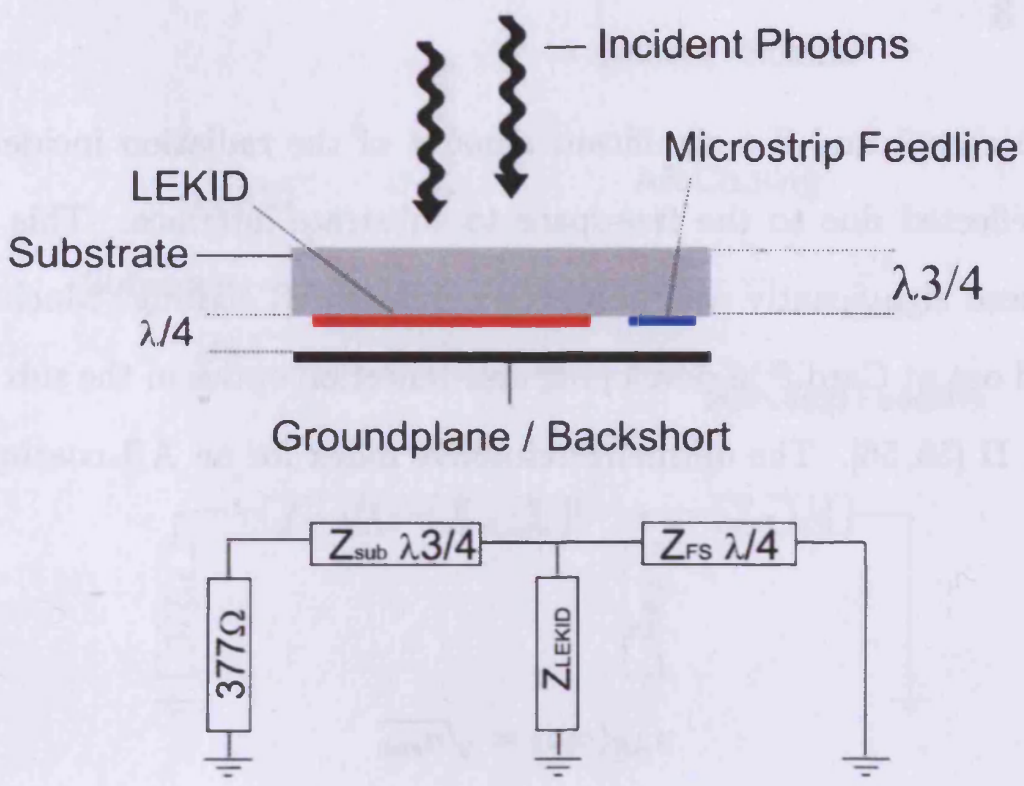

Figure 7.8: (top) Schematic of a LEKID illuminated through the substrate. (bottom) Equivalent transmission line model. Here we have the additional impedance $Z_{F S}$ which represents the free-space impedance $(377 \Omega \mathrm{s})$ between the substrate and the back-short.

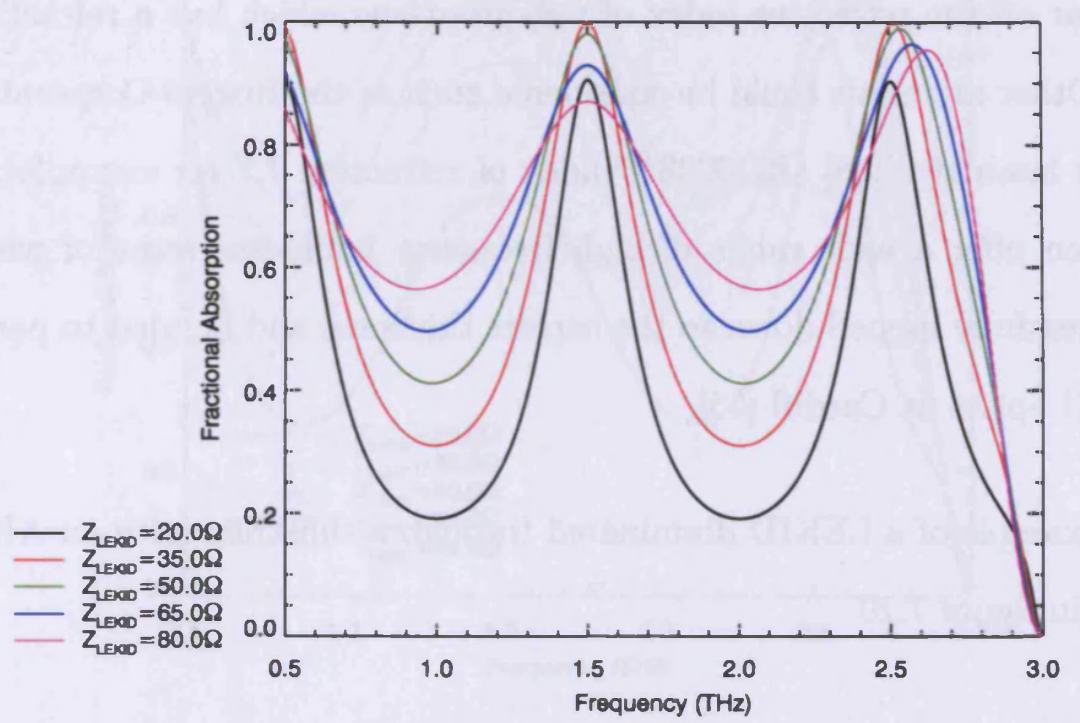

Figure 7.9: Absorption for a LEKID illuminated through the substrate with radiation polarized parallel to the meander. Here sapphire has been used as the substrate material $\left(\epsilon_{r} \approx 10\right)$. 


\section{Case 3}

In both case 1 and 2 a significant amount of the radiation incident on the system is reflected due to the free-space to substrate interface. This reflection can be reduced significantly with the addition of an AR coating. Much work has been carried out at Cardiff in developing anti-reflection optics in the sub $\mathrm{mm}$ band for SCUBA II $[55,56]$. The optimum refractive index for an AR coating is given by $[57]$ :

$$
n_{A R}(o p t)=\sqrt{n_{s u b}}
$$

where $n_{A R}(o p t)$ is the optimum refractive index of the AR coating and $n_{\text {sub }}$ is the refractive index of the substrate. For sapphire and silicon substrates this gives optimum AR coating refractive indices of 1.78 and 1.87 respectively. These are not too far off the refractive index of polypropylene which has a refractive index of 1.48. Other materials could be considered such as the Rogers Corporation High

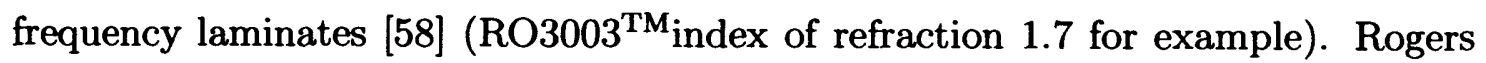
corporation offer a wide range of high frequency laminates some of which have been successfully lapped down to the correct thickness and bonded to parts of the SCUBA II optics at Cardiff [55].

An example of a LEKID illuminated through a substrate with an AR coating is shown in figure 7.10 

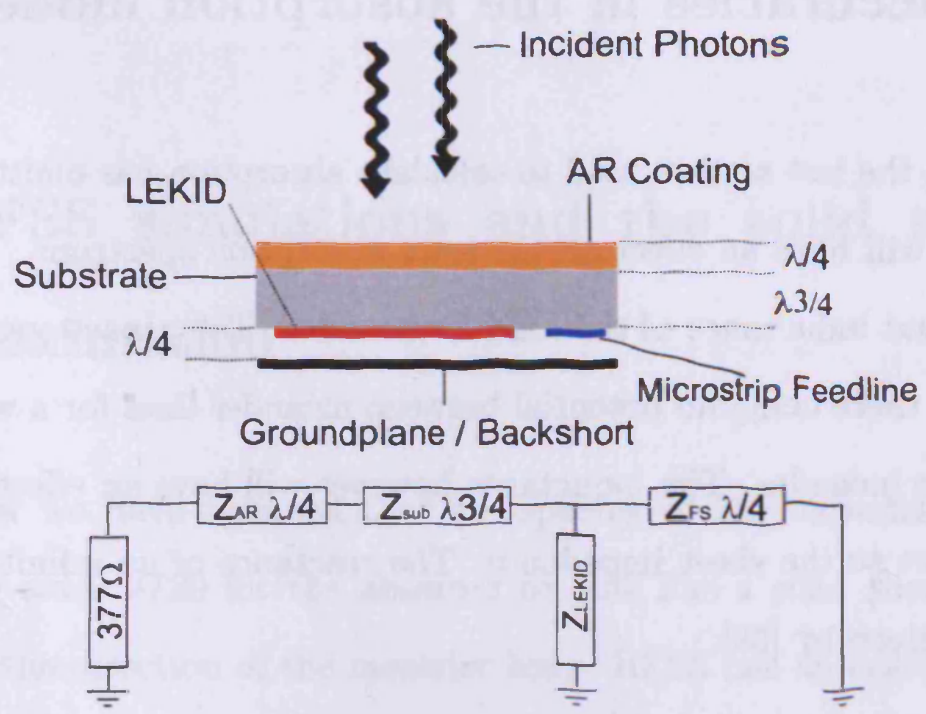

Figure 7.10: (top) Schematic of a LEKID illuminated through the substrate with an AR coating. (bottom) Equivalent transmission line model. Here $Z_{A R}$ is the impedance of the anti-reflection coating.

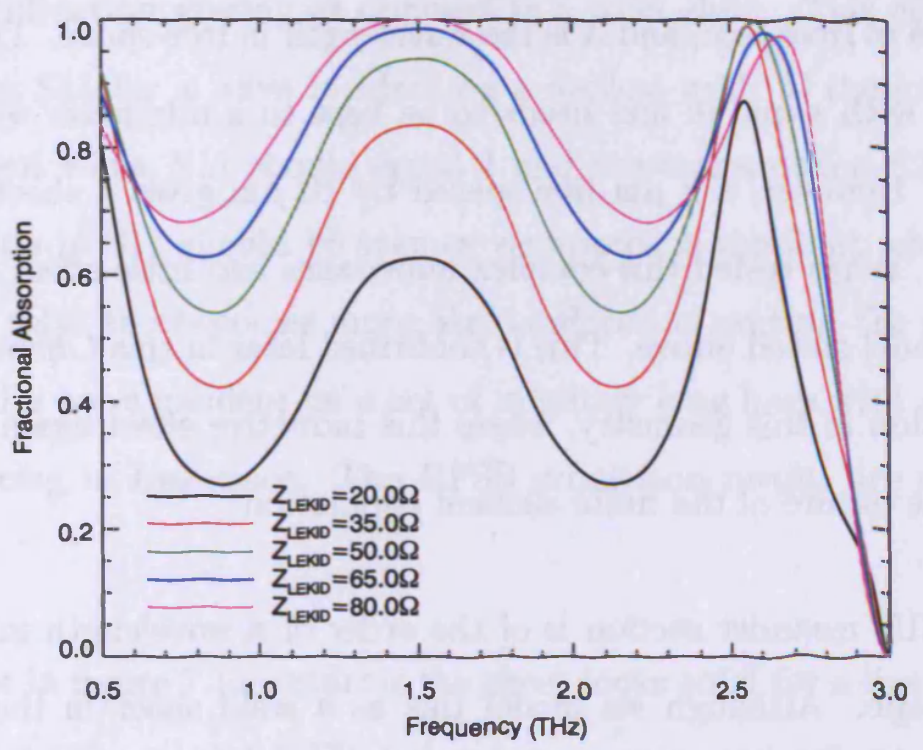

Figure 7.11: Absorption for LEKID illuminated through the substrate with an AR coating by radiation polarized parallel to the meander. Here sapphire has been used as the substrate material $\left(\epsilon_{r} \approx 10\right)$ and polypropylene $\left(\epsilon_{r} \approx 2.2\right)$ has been used for the AR coating. 


\subsection{Inaccuracies in the absorption model}

The model in the last section used to calculate absorption has omitted some subtleties which will have an effect on the total absorption spectrum. These are the capacitance and inductance of the LEKID meander. The capacitance will be negligible due to there being no potential between meander lines for a wave polarised parallel to the meander. The inductance however will have an effect and will add a complex part to the sheet impedance. The reactance of an infinite wire grid in free space is given by [59]:

$$
\frac{X S}{Z_{s}}=\frac{s}{\lambda} \ln \operatorname{cosec}\left(\frac{\pi a}{2 w}\right)
$$

Here, $s$ and $w$ are defined as they were for the effective sheet impedance, $Z_{s}$ is the impedance of freespace, and $\lambda$ is the wavelength in free-space. The inductance clearly varies with $s$ and $w$ and needs to be kept to a minimum when designing the meander. However, a $2 \mu \mathrm{m}$ line spaced by $10 \mu \mathrm{m}$ gives a sheet reactance of arround $10 \Omega$. When tested this complex impedance had little effect on the overall absorption model stated above. This is confirmed later in this Chapter with a full HFSS simulation of this geometry, where this inductive effect again is taken into account by the nature of the finite element simulation.

The LEKID meander section is of the order of a wavelength in size and has a complex shape. Although we model this as a solid sheet in the polarisation parallel to the meander lines this is only truly the case for an infinite sheet size. The true absorption of the detector will depend on the natural modes the detector can couple to [60]. The solution to this again will be very complex due to the complex geometry involved, however this could be solved with the appropriate 
HFSS simulations.

\subsection{HFSS simulations and the solid sheet ap- proximation}

Up until now we have assumed that the spacing of the meander section needs to be of the order $\lambda / 20$ for the absorber to look like a solid sheet to a photon polarised in the direction of the meander lines. HFSS has an option to model an infinite sheet by repeating an input pattern. Simulations were performed on an infinite set of parallel lines to establish the reduction in photon interaction with the meander as the line spacing is increased. In the limit where the solid sheet approximation begins to break down a set of infinitely long parallel lines begins to look like a diffraction grating as opposed to a solid sheet. This can be tested for by measuring S11 for a wave incident on a lossless grid. If the grid apears solid to the incident wave, S11 should equal 1 and the transmission S21 should equal 0. A reduction in $\mathrm{S} 11$ should be seen as we approach the limit where the grid no longer looks solid and becomes more like a diffraction grating. To test this theory we simulated a wave incident on a set of infinitely long lines with a set width but variable spacing in free-space. The HFSS simulation results are shown in figure 7.13

The plot in figure 7.13 confirms the sheet looks solid for a line spacing within the $\lambda / 20$ limit $(10 \mu \mathrm{m}$ at $1.5 \mathrm{THz})$ showing an estimated reflection of more than $97 \%$ of the incident power for a line spacing of $10 \mu \mathrm{m}$. We see a reduction in S11 as we increase the line spacing, however the reduction is gradual and it may well be better to work outside the $\lambda / 20$ limit in order to increase effective sheet impedance 


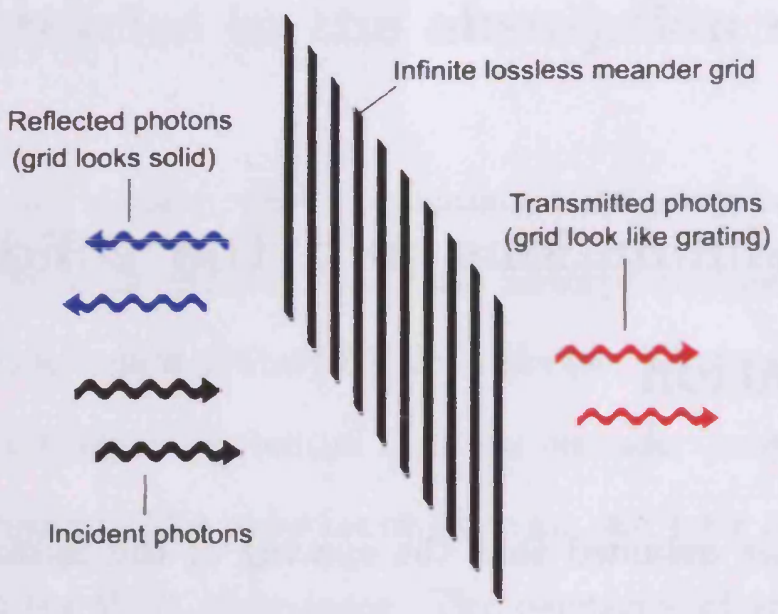

Figure 7.12: HFSS model of the solid sheet approximation. Here the photons that reflect off the grid are measured as S11 and see the sheet as solid.

to improve overall absorption. It is also worth noting that this model only allows for photons to interact with the grid during a single pass. In a real system the back-short and impedance mis-match between substrate and free-space boundaries will introduce multiple passes of the meeander section by a photon. This effect will increase the interaction probability with the meander and hence increase the probability of photon absorption. These simulations are courtesy of Dr Jin Zhang Cardiff University. 


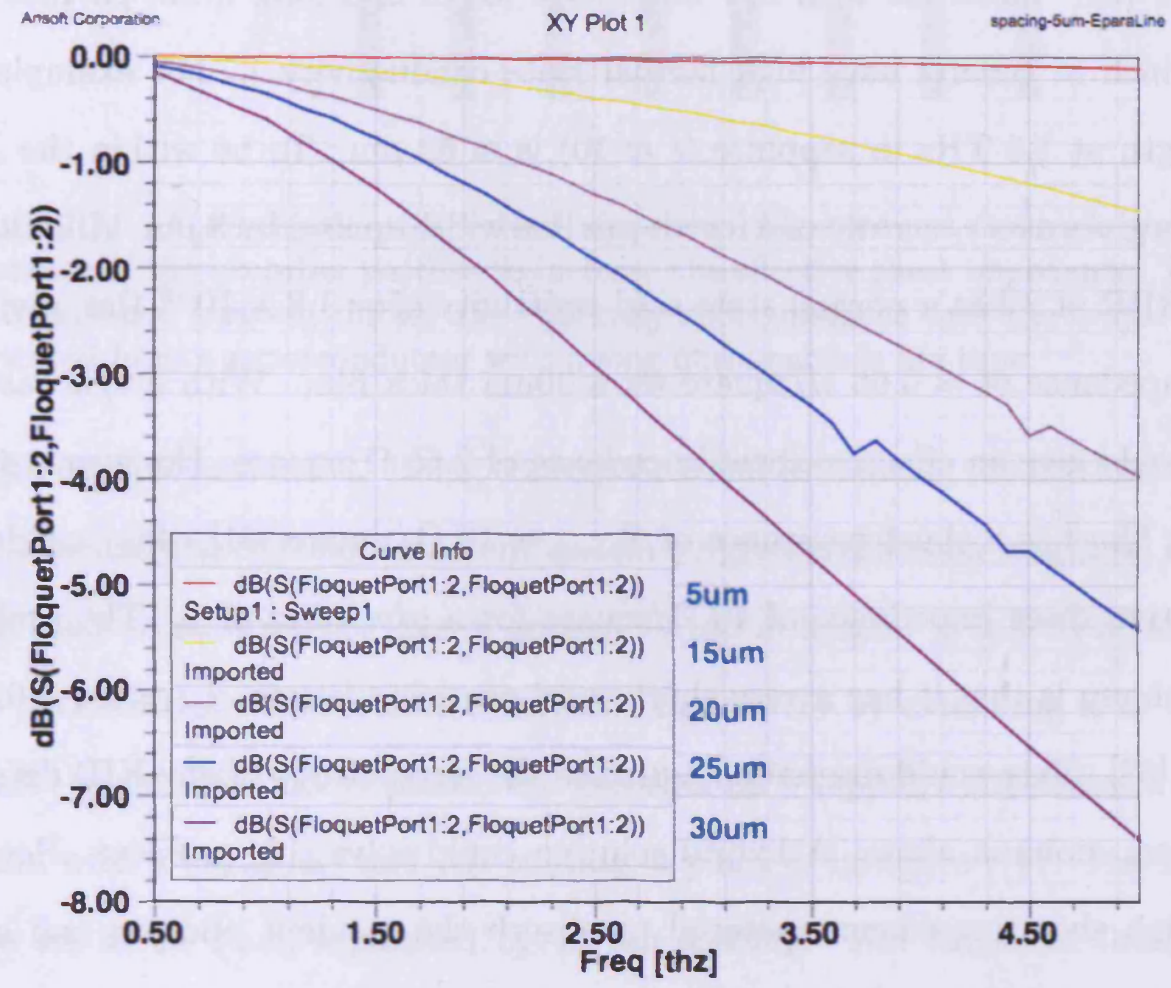

Figure 7.13: HFSS simulations of the solid sheet approximation. This is a plot of S11 vs frequency for a wave incident on a set of $2 \mu \mathrm{m}$ wide parallel lines with varying spacing. As expected a reduction in S11 is seen as we move to higher frequency or wider line spacing. 


\subsection{LEKID Hybrid solutions}

Although the LEKID meander demonstrates a higher effective sheet impedance when compared to that of the a solid sheet, it is still difficult to create a high effective sheet impedance from superconductors which would be practical for KID detectors (i.e. materials with low microwave losses and long quasi-particle lifetimes which in general have high normal state conductivity ). For example the wavelength at $1.5 \mathrm{THz}$ in sapphire $(\epsilon \approx 10)$ is $\approx 63 \mu \mathrm{m}$. To be within the $\lambda / 20$ limit this gives us a $s / w$ ratio of 4 for a $1 \mu \mathrm{m}$ line width spaced by $3 \mu \mathrm{m}$. Aluminium with a $\mathrm{RRR}$ of 2 has a normal state cold resistivity of $\approx 1.3 \times 10^{-8} \Omega \mathrm{m}$, giving a sheet impedance of $\approx 0.66 \Omega /$ square for a $20 \mathrm{~nm}$ thick film. With a $s / w$ ratio of 4 this would give an effective sheet impedance of $2.65 \Omega /$ square. However a $40 \mathrm{~nm}$ niobium film has a sheet resistance of $Z_{\text {sheet }} \approx 10 \Omega /$ square [61], this would give an effective sheet impedance of $40 \Omega$ /square for a s/w ratio of 4 . The problem with niobium is that it has a very short quasi-particle lifetime, $\tau_{q p} \approx 0.4-0.5 \mu \mathrm{s}$ at $1.8 \mathrm{~K}$ [62]. This would dramatically reduce the responsivity of any KID detector made from niobium alone. A hybrid solution could solve this problem. Here we use a high sheet impedance material to absorb the incident photons and allow the quasi-particle generated to diffuse into quasi-particle traps which occupy the space between absorbers in the meander fabricated from a material with a long quasi-particle lifetime. A schematic of such an idea is shown in figure 7.14

The hybrid solution relies on the quasi-particle diffusion length being long enough in the absorber material to be able to diffuse into the trapping material. niobium measured at $1.8 \mathrm{~K}$ has a diffusion constant of $7 \mathrm{~cm}^{2} / s$ for a RRR of 5 [62]. Assuming that a high resistance $40 \mathrm{~nm}$ niobium film has a reduced RRR and hence diffusion constant, along with a shorter lifetime, we can calculate the diffusion 


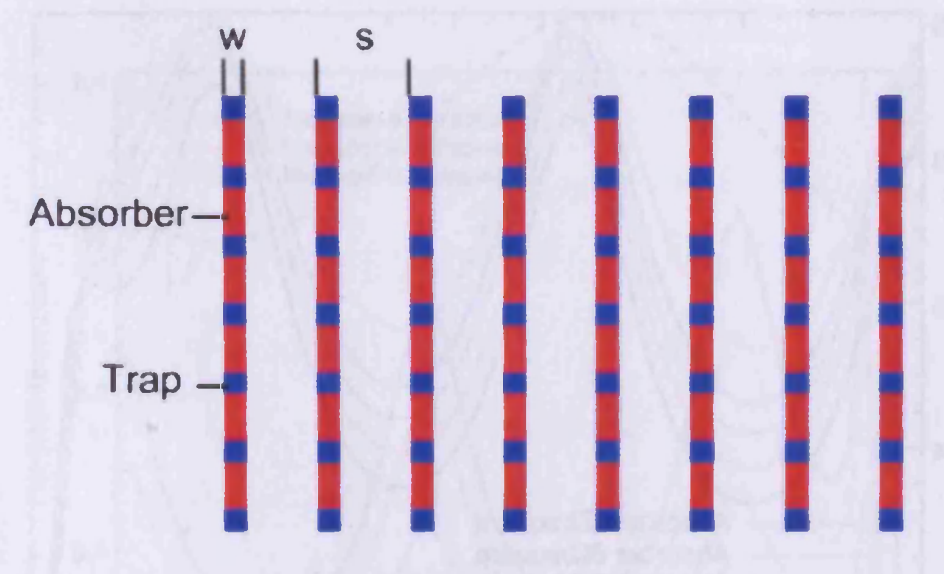

Figure 7.14: A schematic of a hybrid absorber. Here we use the high normal state resistance of the absorber patches to increase the effective sheet impedance. The quasiparticles, once created, diffuse the relatively short distance into the traps which are fabricated from a superconductor with a long quasi-particle life-time.

length using equation 7.1. Being conservative and estimating the lifetime at $100 \mathrm{mK}$ as $0.1 \mu \mathrm{s}$ and the diffusion constant as $3 \mathrm{~cm}^{2} / s$ gives a diffusion length of, $l \approx 5 \mu \mathrm{m}$. This leads to an absorber size of around $10 \mu \mathrm{m}$ in length. A full HFSS simulation was performed on the structure shown in figure 7.14 by extending it to exist over infinite space. The model placed the absorber on a sapphire substrate of thickness $45 \mu \mathrm{m}$ with a backshort and AR coating in place as in figure 7.11. The line width were set to be $2 \mu \mathrm{m}$ separated by $10 \mu \mathrm{m}$ spacing. The length of the absorbing patches was set to $10 \mu \mathrm{m}$ and the length of the traps $2 \mu \mathrm{m}$. The results are shown in plot 7.15 .

The HFSS simulations indicate that working outside the $\lambda / 20$ limit for sapphire is not an issue and should be considered to achieve a high effective sheet impedance of the meander section. The simulations indicate that using a hybrid solution, over $80 \%$ absorption could be achieved over a $0.5 \mathrm{THz}$ band using a absorber normal state impedance of $10 \Omega /$ square on a $3 \lambda / 4$ substrate. This band is broadened to around over $80 \%$ absorption across a $1.75 \mathrm{THz}$ band when using a 


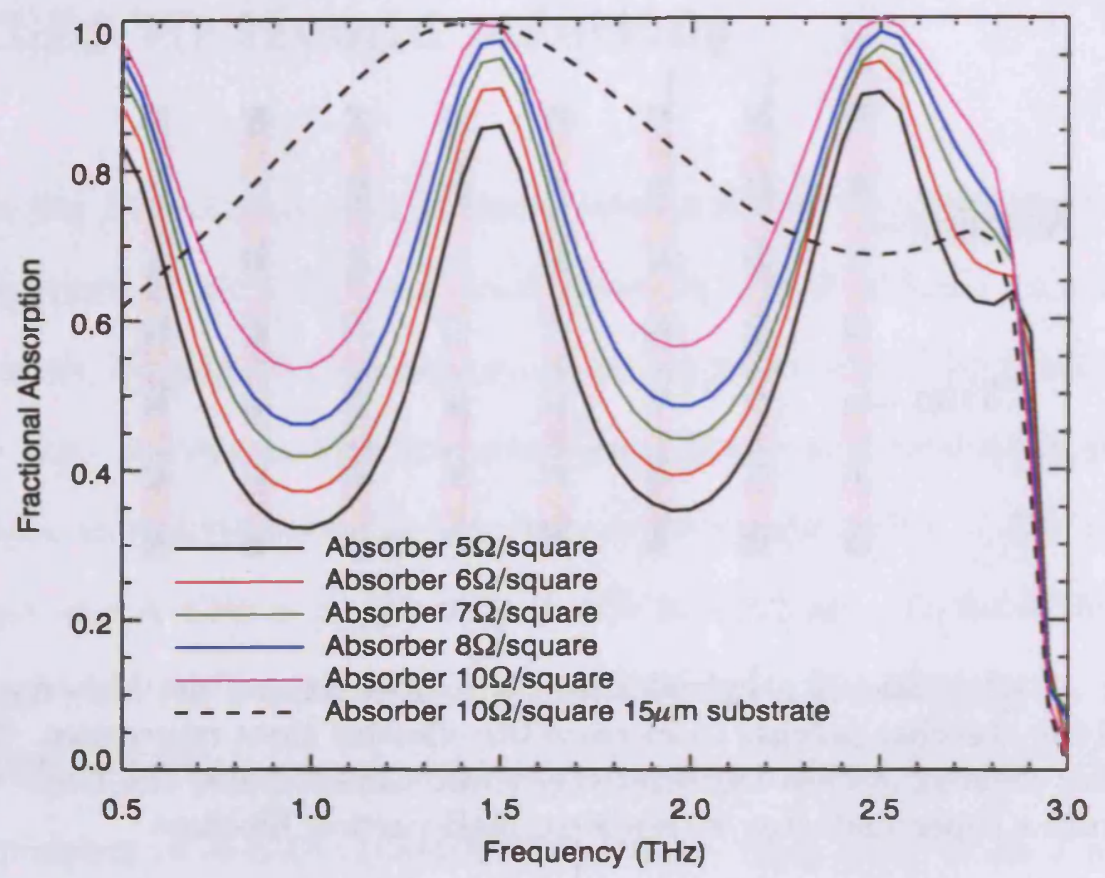

Figure 7.15: HFSS simulation of a hybrid LEKID on a $3 \lambda / 4$ substrate with $2 \mu \mathrm{m}$ line widths spaced by $10 \mu \mathrm{m}$. The last data set over plotted is for a $\lambda / 4$ substrate. Here we an increase in the absorption bandwidth of the meander section. Simulations courtesy of Dr Jin Zhang Cardiff University.

$\lambda / 4$ thick substrate. The model used in figure 7.15 was simulated for meanders fabricated from a single high normal state conductivity metal on substrates with a thickness of $3 \lambda / 4$ and $\lambda / 4$. The results which can be seen in figures 7.16 and 7.17 show that between 10 and $30 \%$ absorption could be achieved with a single material LEKID with the bandwidth being broadened using the $\lambda / 4$ substrate. The $\lambda / 4$ substrate also demonstrates a resonance effect at around $3 \mathrm{THz}$ where the absorption reaches as high as $95 \%$. This feature could be exploited for narrow band photometry using ground based instruments at $200 \mu \mathrm{m}$ where only a narrow atmospheric window exists. 


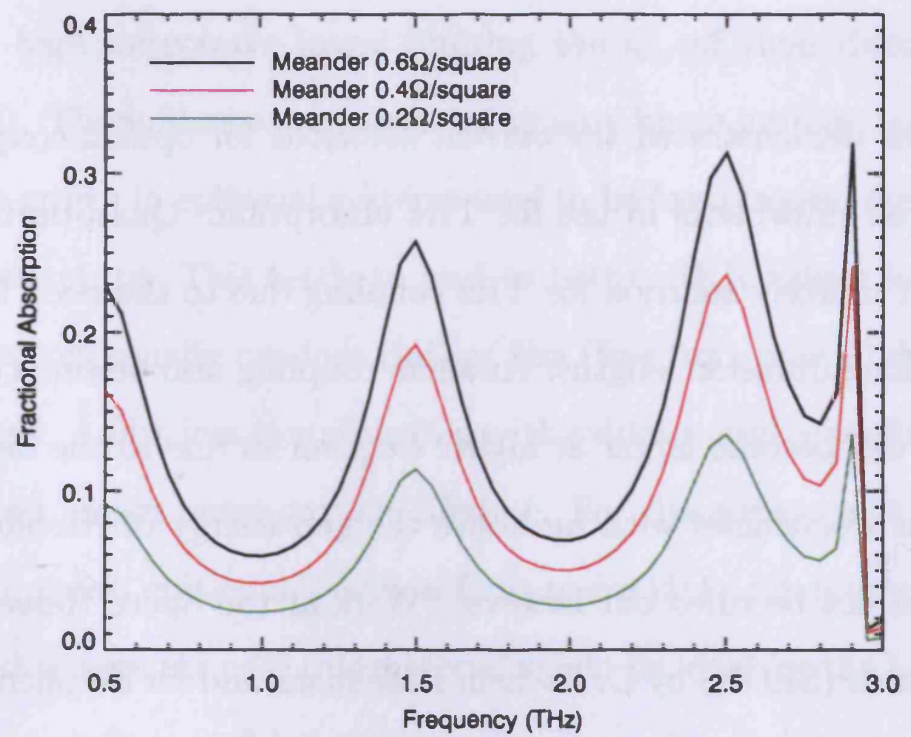

Figure 7.16: HFSS simulation of a single material LEKID on a $3 \lambda / 4$ substrate with $2 \mu \mathrm{m}$ line widths spaced by $10 \mu \mathrm{m}$. Simulations courtesy of Dr Jin Zhang Cardiff University.

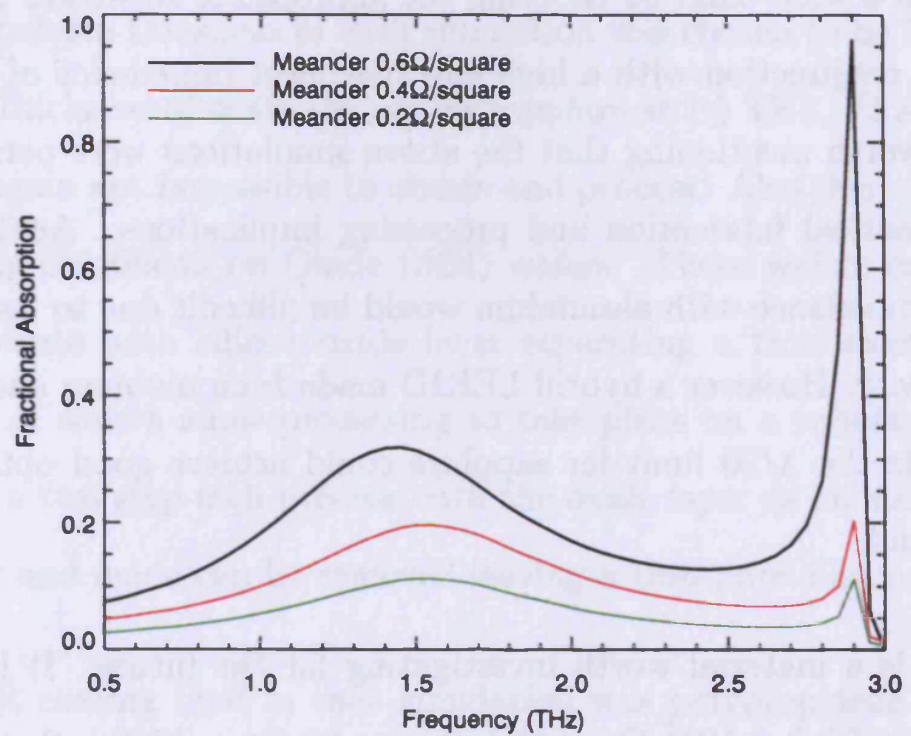

Figure 7.17: HFSS simulation of a single material LEKID on a $\lambda / 4$ substrate with $2 \mu \mathrm{m}$ line widths spaced by $10 \mu \mathrm{m}$. Simulations courtesy of Dr Jin Zhang Cardiff University. 


\subsection{Optical coupling conclusion}

This section has demonstrated the current solutions for optical coupling to KID devices and their drawbacks in use for $\mathrm{THz}$ absorption. Quasi-particle trapping seems the most unlikely solution for $\mathrm{THz}$ coupling due to the need for extremely thin film with long diffusion lengths. Antenna coupling also presents difficulties in general which will become larger at higher frequencies due to the losses in transmission lines at frequencies with $h \nu$ above the gap energy of Niobium. Antenna coupling should not be ruled out however. Work at the Space Research Institute of the Netherlands (SRON) by Dr Jochem Baselmans and Dr Stephen Yates is still ongoing to develop an antenna solution suitable for THz absorption. The LEKID direct absorption method looks very promising. The fact that there is no need to transport the photon energy once absorbed to a detector simplifies the problem dramatically. As seen from the simulations above, good optical coupling can be achieved across a wide band by choosing the appropriate substrate thickness and AR coating in conjunction with a high effective sheet impedance of the meander section. It is worth mentioning that the above simulations were performed while considering practical fabrication and processing implications. Achieving a high effective film impedance with aluminium would be difficult due to its high normal state conductivity. However a hybrid LEKID made from niobium and aluminium, working outside the $\lambda / 20$ limit for sapphire could achieve good optical coupling over a wide band.

Titanium is a material worth investigating for the future. It has a normal state resistivity of $7.8 \times 10^{-7} \Omega \mathrm{m}$ which is over 20 times higher than aluminium. Titanium also has an extremely long quasi-particle life time, over $5 \mu$ s at 100 $\mathrm{mK}$ [51] which is two orders of magnitude longer than the best aluminium films 
at the same temperature. Tests by SRON on titanium to date unfortunately show that it has high microwave losses limiting the $Q_{u}$ of their distributed KIDs to around 1000. These films were grown using an e-beam system, which may not be ideal. Films grown in epitaxial systems tend to be less stressed due to the elevated growth temperature. This tends to lead to better RRR values for the film. Also epitaxial systems usually produce cleaner film (free from oxides) due to their lower base pressures. A film less contaminated with oxides would most likely have a lower resistivity and longer quasi-particle lifetime. For these reasons it would be worth experimenting with epitaxial titanium films to see if the microwave losses could be reduced. If this were the case this material would be ideal for the LEKID and could even be used in a "reverse" hybrid scenario, where the absorbing patches are small but also act as the quasi-particle traps and the rest of the meander is completed with a low microwave loss material (thick niobium for example) to increase the un-loaded quality factor.

The substrate thickness in each simulation was chosen to be $3 \lambda / 4$. This gives a substrate thickness of $\approx 45-50 \mu \mathrm{m}$ for sapphire at $1.5 \mathrm{THz}$. This is indeed a thin wafer, but again not impossible to obtain and process. Also there is the possibility of processing on Silicon on Oxide (SOI) wafers. These wafers consist of a thick silicon substrate with silicon oxide layer separating a thin second silicon layer. These type of wafers allow processing to take place on a robust thick wafer and then, using a two step etch process with the oxide layer as an etch stop, the bulk silicon layer and oxide can be removed leaving a thin pure silicon substrate [63].

The AR coating used in each simulation was polypropylene. This is not an optimum AR coating for silicon or sapphire but is very easy to process and bond to substrates. The Rogers high frequency laminates could be used and would be closer to an optimum index of refraction for both silicon and sapphire. However 
these materials are ridged and will be difficult to lap down to a thickness of $\lambda / 4$ while retaining a good surface roughness at $1.5 \mathrm{THz}$. However neither AR coating seems an unreasonable solution from a processing point of view.

The LEKID presents many solutions for optical coupling which makes it an attractive option for using KID devices at high frequency. Although the focus through this chapter has been on $1.5 \mathrm{THz}$ absorption it should be noted that coupling a LEKID to free space becomes easier as we move to longer wavelengths and the $\lambda / 20$ limit becomes larger. Working with a larger LEKID meander would make coupling to other astronomical bands of interest from the ground possible. These are the atmospheric windows at $350 \mu \mathrm{m}$ and $450 \mu \mathrm{m}$. 


\section{Chapter 8}

\section{Cardiff experimental set up}

All the initial testing to demonstrate the proof of concept of the LEKID device was performed at Cardiff using a wet $4 \mathrm{~K}$ cryostat and sorption cooler. Further testing at lower temperatures with a full noise analysis test set up was performed at SRON and will be mentioned later in this report.

\subsection{LEKID chip packaging}

The standard substrate used for LEKID fabrication was sapphire of dimensions $12 \times 12 \mathrm{~mm}$ and thickness $325 \mu \mathrm{m}$. This was bonded to a chip carrier using silver epoxy by EPO-TEK $®[64]$ to make the electrical connection to the ground-plane. The material beneath the part of the chip containing the LEKID resonators of the chip carrier was removed. This was done to avoid the ground-plane under the resonators proximitising with the normal metal of the chip carrier and inducing losses. The microwave probe signal is launched onto the chip via a set of wire 
bonds from a grounded coplanar section bonded to the chip carrier and soldered to an SMA connector. A schematic of the chip carrier along with a photo of a mounted device can be seen in figure 8.1.
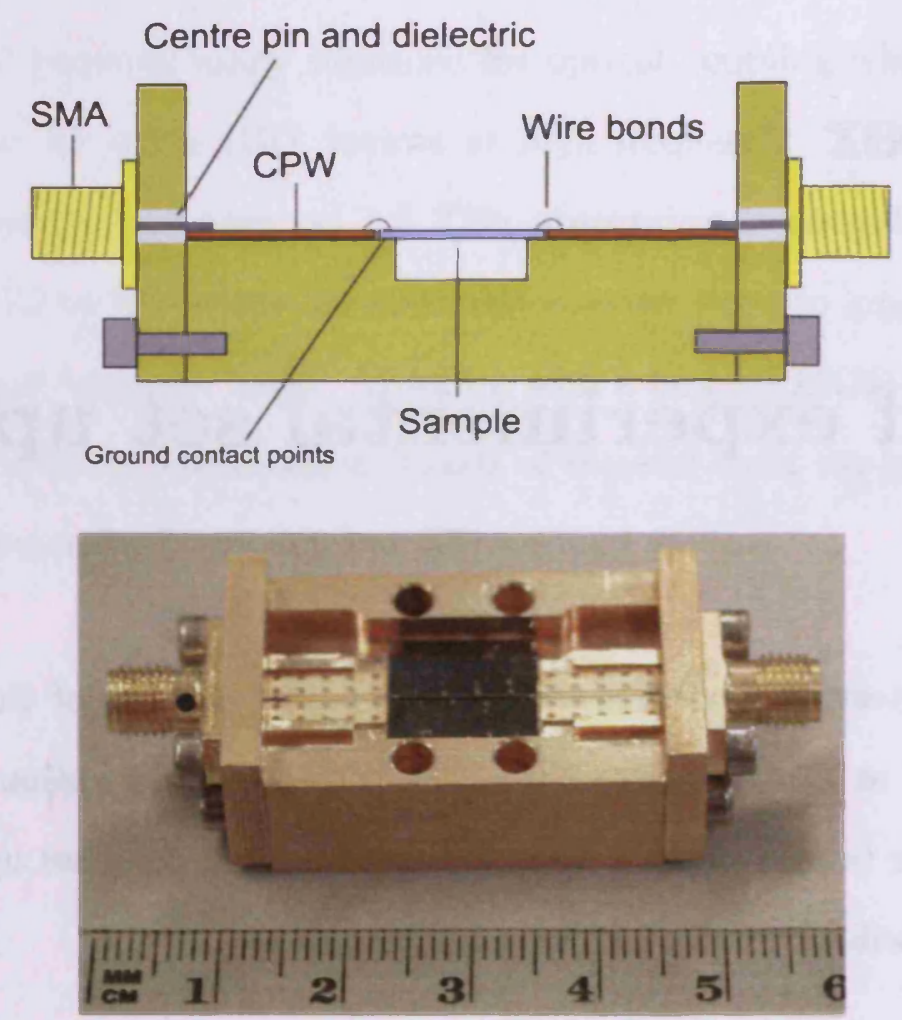

Figure 8.1: (top) A schematic cross section of the LEKID chip carrier. (bottom) A photo of a mounted LEKID sample

The silver epoxy needs to be cured at a temperature of $50^{\circ} \mathrm{C}$ or higher. This was performed in a vacuum oven to minimise the oxidation of the film. The grounded coplanar sections were designed in Sonnet and fabricated by Tru-lon. Tru-lon have the capabilities of producing via holes (seen in the photo of figure 8.1) joining the ground-planes on the top and bottom surfaces of the grounded coplanar sections. This action prevents undesired modes propagating along the coplanar lines. 


\subsection{Low temperature system}

The initial testing at Cardiff was performed in a Janis liquid helium cryostat [65] fitted with two Chase Cryogenic sorption coolers [66]. This system had a base temperature of $\approx 280 \mathrm{mK}$. The ultra-cold part of this system actually comprised two separate sorption coolers coupled together to reach the final base temperature. The two sorption coolers used were previously used in other experiments at Cardiff and had since become redundant. The first cooler was previously used as the precooler for a miniature dilution refrigerator and had a base temperature of around $350 \mathrm{mK}$. This cooler consisted of a ${ }^{4} \mathrm{He}$ pump and still used to pre-cool the ultacold head to condense ${ }^{3} \mathrm{He}$ from a second pump. The second sorption cooler was designed to operate from a pumped liquid helium cryostat providing a $1.5 \mathrm{~K}$ stage. This was a single stage cooler consisting of a single ${ }^{3} \mathrm{He}$ pump and still. The system used in our experiments used the two stage cooler to pre-cool the single stage cooler down to a temperature where condensation of ${ }^{3} \mathrm{He}$ was possible. Once the single stage ${ }^{3} \mathrm{He}$ cooler had cycled the first cooler was used to pre-cool the cables and tubes connecting the ultra-cold head to the $4 \mathrm{~K}$ base plate in order to reduce the loading on the ultra-cold stage. A schematic diagram of this system can be seen in figure 8.2

Sorption coolers can only be loaded with a few micro-watts of thermal power, so thermal loading should be kept to a minimum in order to reduce the base temperature and increase the hold time of the system. In order to keep the thermal load on the UC stage to a minimum thin Cupronickel coaxial cables were used to carry the microwave probe signal to the chip carrier. The cross-sectional area of the combined inner and outer conductors of these cables is $\approx 6.6 \times 10^{-3} \mathrm{~cm}$ [67]. The measured thermal properties of these cables by Kushino, Ohkubo and Fujioka [68] 


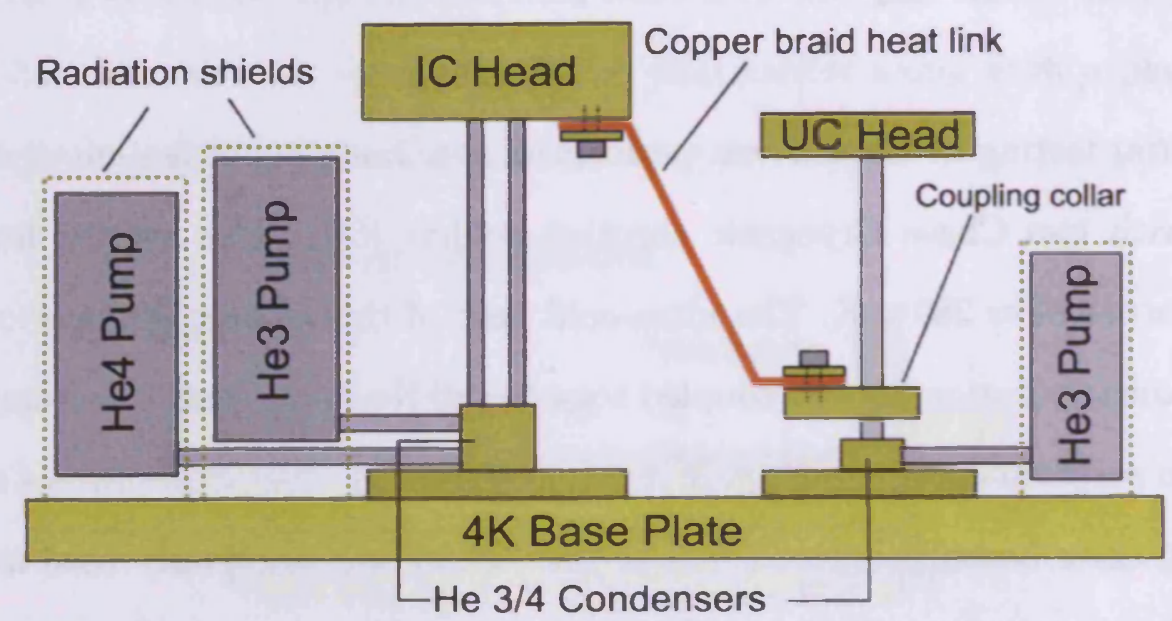

Figure 8.2: A schematic of the low temperature set up. The first set of coolers is used to pre-cool the single ${ }^{3} \mathrm{He}$ cooler and is then used as an intermediate (IC) temperature stage to pre-cool the cable running to the ultra-cold (UC) stage. The chip carrier shown in figure 8.1 is bolted directly to the UC stage

indicate that for our cables, heat sunk at the IC stage, and with $10 \mathrm{~cm}$ of cable length between the IC and UC stage, we should expect less that a micro-watt of thermal power input on the UC stage. The base temperature of this system was $282 \mathrm{mK}$ and had a hold time of around 8 hours with the IC stage running out first. This hold time is quite poor for a large sorption cooler and is due to the large amounts of loading from the point at where the IC stage is connected to the UC cooler. Here only about $15 \mathrm{~mm}$ of thin-walled stainless-steel tubing separates the IC head from the $4.2 \mathrm{~K}$ base plate. Although the hold time is short it is sufficient to carry out a days worth of testing, and is not bad for a system never intended to be operated in this manner.

One problem when working with coaxial cable at low temperature is thermalising the centre conductor. The centre conductor is quite well insulated by the PTFE dielectric that surrounds it. In order to aid the thermalisation of the centre conductors, DC blocks we added to the cables between $4.2 \mathrm{~K}$ and the IC stage and 
between the IC stage and UC stage. The DC blocks add a thermal impedance to both the inner and outer conductors while leaving the line electrical impedance more or less unchanged at $\mathrm{GHz}$ frequencies. In addition to this a $20 \mathrm{~dB}$ microwave attenuator was added on the microwave input cable between $4.2 \mathrm{~K}$ and the IC stage. These actions, as well as ensuring good heat sinking of the outer conductor at each stage, help to make sure the inner conductor is thermalised and does not transport unnecessary heat to the UC stage and LEKID chip.

Between $4.2 \mathrm{~K}$ and room temperature a set of RG-405 stainless steel semiridged coaxial lines were used. RG-405 is a standard cable dimension and the cables were made up at Cardiff using a traditional SMA connector kit. In order to solder to the stainless-steel cables to SMA connectors, Duzzel@ flux was used to first "wet" the surfaces to be soldered. Again to ensure low heat loading and hence low liquid helium boil-off these cables were heat sunk to the liquid nitrogen can at $77 \mathrm{~K}$. The cables were connected to pair of hermetic SMA connectors at the top of the cryostat. Each cable and connector in this cold RF chain was swept individually and together with the VNA to ensure each was functioning properly. Where applicable, DC tests we also carried out to test for shorts between the centre conductor and ground. Figure 8.3 shows a photograph of the cryostat cold plate with the coolers and cold RF components in place.

\subsection{Cooler control and thermometry}

The running of the three sorption cooler is quite a lengthy process and involves varying the power and activating heat switches of the three associated pumps at various times throughout the cycle. The total time taken for the cycle is around two 


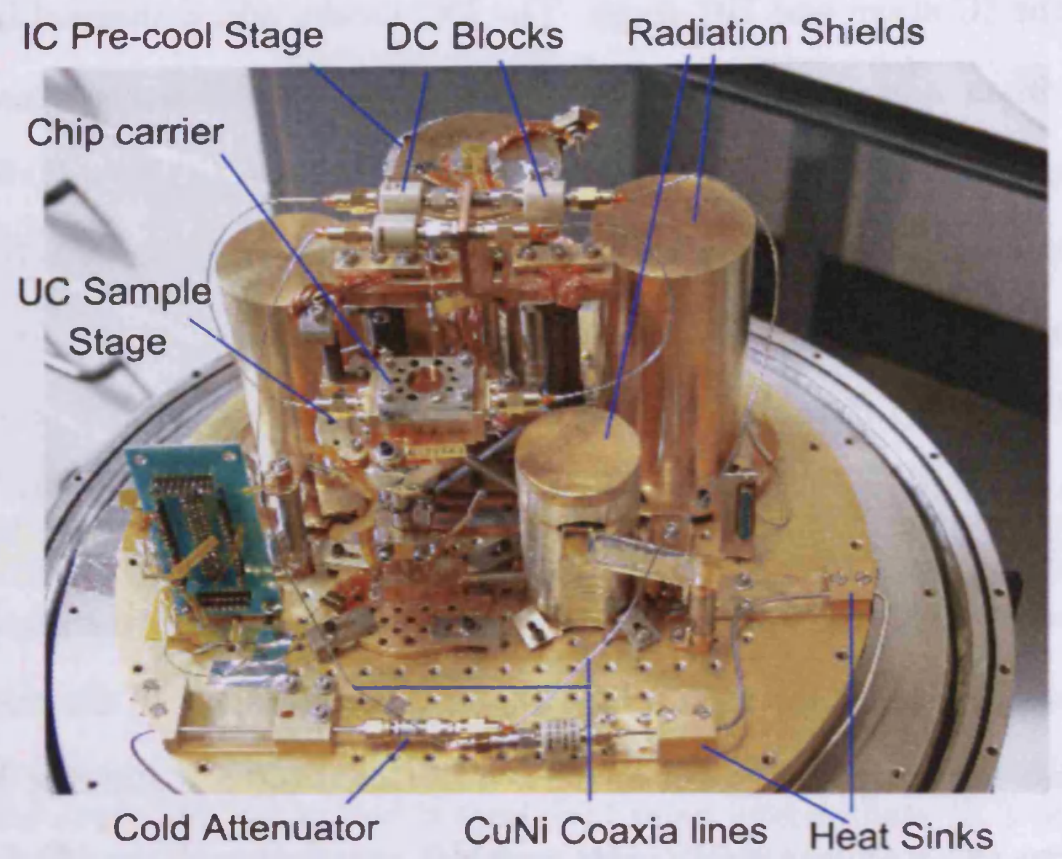

Figure 8.3: Photograph of the test cryostat with a sample in place on the UC stage.

and a half hours so an automatic cycling procedure was written in XML code and executed by a QMCI TC40/1 temperature and control system [69]. This system consists of an eight channel temperature diode readout along with four amplified low power thermometry readout channels. The unit also has six voltage output channels which can be programmed, using XML code, to vary the output voltage on each. The voltage outputs can be triggered using time intervals or data from any of the temperature readout channels. The basic cooler cycle is outlined in table 8.1.

The cycle procedure is designed to make sure of the following:

- That all the ${ }^{4} \mathrm{He}$ from the first pump has condensed. This can be ensured by noting a slight rise in the pump temperature once all the helium has been condensed. The cycle procedure waits a pre-determined time for this 
Table 8.1: Cooler cycle procedure

\begin{tabular}{lllllll}
\hline \hline Action & ${ }^{4} \mathrm{He}$ pump 1 & ${ }^{3} \mathrm{He}$ pump 1 & ${ }^{3} \mathrm{He}$ pump 2 & HS 1 & HS 2 & HS 3 \\
\hline Start cycle & $25 \mathrm{~V}$ & $25 \mathrm{~V}$ & $8.5 \mathrm{~V}$ & $0 \mathrm{~V}$ & $0 \mathrm{~V}$ & $0 \mathrm{~V}$ \\
Stabilise ${ }^{3} \mathrm{He} \mathrm{1}$ & $25 \mathrm{~V}$ & $10.5 \mathrm{~V}$ & $8.5 \mathrm{~V}$ & $0 \mathrm{~V}$ & $0 \mathrm{~V}$ & $0 \mathrm{~V}$ \\
Stabilise ${ }^{4} \mathrm{He} \mathrm{1}$ & $11 \mathrm{~V}$ & $10.5 \mathrm{~V}$ & $8.5 \mathrm{~V}$ & $0 \mathrm{~V}$ & $0 \mathrm{~V}$ & $0 \mathrm{~V}$ \\
Wait ${ }^{4} \mathrm{He}$ condense & $11 \mathrm{~V}$ & $10.5 \mathrm{~V}$ & $8.5 \mathrm{~V}$ & $0 \mathrm{~V}$ & $0 \mathrm{~V}$ & $0 \mathrm{~V}$ \\
Pump ${ }^{4} \mathrm{He} \mathrm{1}$ & $0 \mathrm{~V}$ & $10.5 \mathrm{~V}$ & $8.5 \mathrm{~V}$ & $5 \mathrm{~V}$ & $0 \mathrm{~V}$ & $0 \mathrm{~V}$ \\
Wait ${ }^{4} \mathrm{He} \mathrm{1} \mathrm{runout}$ & $0 \mathrm{~V}$ & $10.5 \mathrm{~V}$ & $8.5 \mathrm{~V}$ & $5 \mathrm{~V}$ & $0 \mathrm{~V}$ & $0 \mathrm{~V}$ \\
Pump ${ }^{3} \mathrm{He} \mathrm{1} \mathrm{\&} \mathrm{2}$ & $0 \mathrm{~V}$ & $0 \mathrm{~V}$ & $0 \mathrm{~V}$ & $5 \mathrm{~V}$ & $5 \mathrm{~V}$ & $5 \mathrm{~V}$ \\
Cycle Ended & $0 \mathrm{~V}$ & $0 \mathrm{~V}$ & $0 \mathrm{~V}$ & $3.5 \mathrm{~V}$ & $3.5 \mathrm{~V}$ & $3.5 \mathrm{~V}$ \\
\hline HS =Heat switch & & & & & &
\end{tabular}

to happen.

- That all the condensed ${ }^{4} \mathrm{He}$ has been exhausted before pumping on the condensed ${ }^{3} \mathrm{He}$ from pumps 2 and 3 . If pumps 2 and 3 are cycled before pump 1 has run out we run the risk of any leftover ${ }^{4} \mathrm{He}$ turning super-fluid. Superfluid ${ }^{4} \mathrm{He}$ can climb the pumping lines to the still and create a thermal short to the $4 \mathrm{~K}$ plate. To ensure this does not happen the cycle waits for a slight rise in the UC and IC heads indicating the ${ }^{4} \mathrm{He}$ has run out before pumping on the $2{ }^{3} \mathrm{He}$ stills.

- That each heat switch is operated in conjunction with the pumps being turned off and on.

- That the heat-switch ON voltage is reduced once the cycle has completed to reduce radiation loading on the IC and UC stages.

The thermometry for the $4 \mathrm{~K}$ stage and the three sorption pumps were read out using a standard diode and the QMCI TC40/1 diode read channels. A fifth diode was added to the cooler base plate to monitor that it was thermalised properly with the $4 \mathrm{~K}$ plate. The UC and IC stages thermometry consisted of a calibrated Lakeshore Germanium Resistance Thermometer (GRT) [70] and a set of Ruthenium Oxide (RuOx) thermometers. Over several runs the ruthenium oxide 
thermometers were each calibrated against the Lakeshore GRT. As mentioned before, the loading on the UC and IC stages of the cooler needs to be low. These temperature sensors can have a resistance as high as $7 k \Omega$ at $300 \mathrm{~m} \mathrm{~K}$ and therefore need to be read out with low bias currents to prevent loading. The sensors also need to be read out using a 4-wire method to exclude the cable resistance (which can be hundreds of ohms for the low thermal conductivity, thin manganin wires used to wire the IC and UC stages). The QMCI TC40/1 can read out thermometry using a 4-wire method with bias currents of the order of nano-Amps. This is done by adding an RF sealed box housing a set of low noise pre-amplifiers mounted on the cryostat. The electronics within the box generates the small bias currents required to probe the thermometry. This is amplified before being sent to the main control unit to minimise the effect of RF pickup in the cable connecting to the cryostat.

The QMCI TC40/1 unit is also web based. It contains a micro-processor which can be assigned an IP address. This means the box can be controlled from off-site to initiate or monitor cooler cycles. This unit can also store and display thermometer calibration data, fitting the resistance data from the sensors to a set of resistance-temperature data points using a standard spline fit. In general this option was not used and the raw resistance data was sent to a Labview program controlling the rest of the experiment. The XML code used to cycle the cooler was also give a time delay option. This was very useful for starting a cycle before the start of the day, giving the system time to settle before measurements were made. 


\subsection{Microwave readout electronics}

The high frequency electronics of our system was built around a Wlitron 37692B vector network analyser (VNA). This unit was used for sweeping the LEKID devices to measure resonance curves and was also used in continuous wave mode (CW mode) to act a single frequency synthesiser when measuring the optical response of the LEKID to a blackbody source. The typical power needed at chip level to probe KID devices is around -40 to $-50 \mathrm{dBm}$ for niobium and -50 to $-100 \mathrm{dBm}$ for aluminium. The maximum power a KID device can handle before being driven normal is very much dependent on the material, thickness and coupling. For this reason the system was designed to allow the input microwave probe power to be varied.

The basic RF chain can be adapted by adding or removing cable shorts to the RF box when using the VNA in sweep mode or CW (mixer mode). A schematic of the RF chain is shown in figure 8.4. The RF box was designed for ease of use when switching between sweeping and mixing modes. When operating in a sweeping mode the RF output is connected to the second port of the VNA and makes use of the RF amplifier housed within the RF box. To switch to the mixing mode, the RF amplifier output is connected to the RF input of the IQ mixer. The IQ mixer mixes two copies of the RF input with the LO input. One copy is directly mixed and and provides the in-phase mixed output (I). The second copy is mixed with a $90^{\circ}$ phase shift between the LO and RF ports and provides the quadrature mixed output (Q). Redundant RF ports are terminated in either mode. The RF box in mixing mode can allow the I and Q signal to be read directly or amplified by connecting the low frequency amplifiers to the I and Q outputs via a short BNC cable. This option is useful when reading I and Q through an un-amplified Data 
Acquisition system (DAQ). Figure 8.5 shows a photograph of the warm electronics set up.
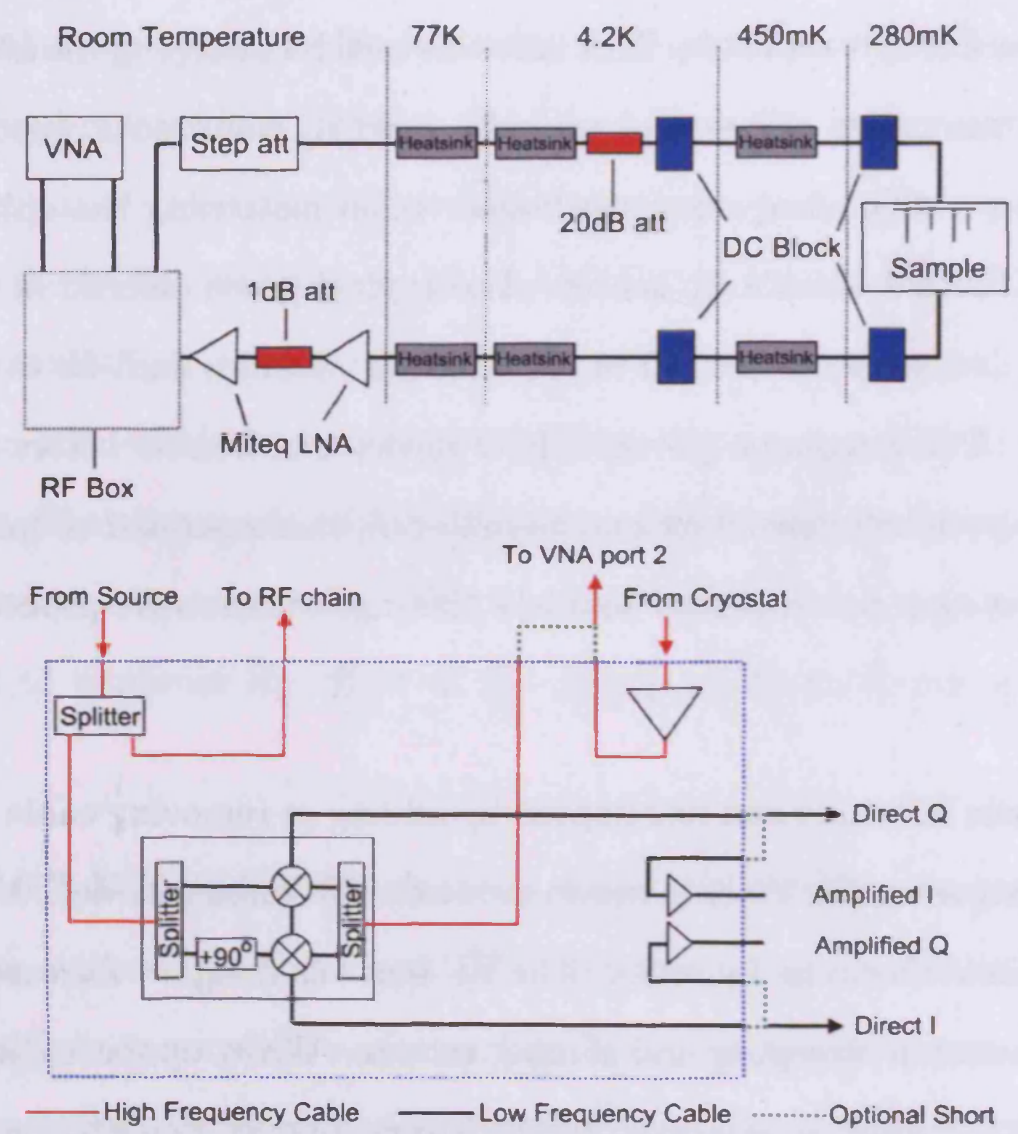

Figure 8.4: (top) A schematic of the RF chain at the various temperature stages through which it passes. (bottom) A schematic of the RF box electronics. 


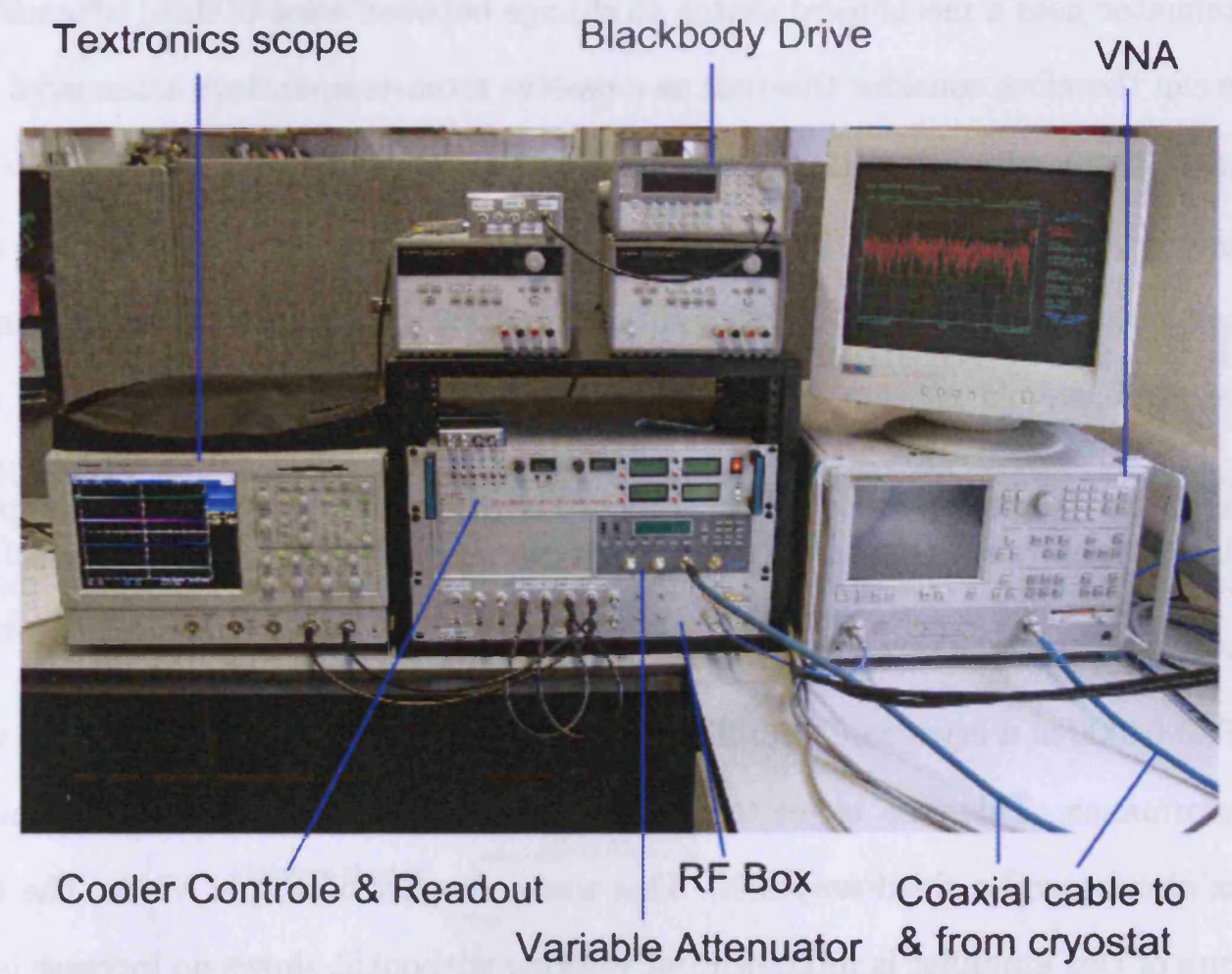

Figure 8.5: (top) Warm readout electronics

\subsection{RF components}

\subsubsection{Variable step attenuator}

The variable step attenuator is used to vary the input power to the entire $R F$ chain. This unit is a Aeroflex-Weinschel 8310 [71]. The variable step attenuator has two channels, each having a attenuation range of $0-62 \mathrm{~dB}$ in steps of $2 \mathrm{~dB}$. The entire unit can be controlled via GPIB allowing pre-set attenuation levels to be defined for each sweep. This is an extremely reproducible approach to adding warm attenuation to the system. The alternative would be to use in-line fixed attenuators. This approach is less reliable and time consuming. The variable step 
attenuator uses a mechanical switch to change between a set of fixed attenuators. We can therefore consider this unit as a passive room-temperature attenuator with a noise figure value equal to that of the attenuation value in $\mathrm{dB}[40]$.

\subsubsection{RF Amplifiers}

The entire RF system has $3 \mathrm{RF}$ amplifiers. The most important is the first amplifier after the sample. The first two amplifiers are Miteq low noise amplifiers models AFS3-04000 and AFS4-00100800-14-10P-4 each with noise figures of 0.8. The AFS3-04000 is a cryogenic amplifier and can be mounted at $77 \mathrm{~K}$ for better noise performance. This step is yet to be done on the current Cardiff set up. The RF box also housed a third amplifier. This was once part of an old VNA. The noise figure of this amplifier is unknown but working without it shows no increase in the noise performance of our system.

\subsubsection{IQ Mixer}

The RF box houses an RF mixer. This is intended to be used for optical and noise measurements of the LEKID devices. The mixer is a Miteq IRM0118LC1Q $2-18 \mathrm{GHz}$ IQ mixer. This is used in a homodyne set up where the RF and LO ports are driven by the same signal split at the source. One copy of the signal is sent directly to the LO port of the mixer. The second copy of the signal is sent through the RF chain shown in figure 8.4 and into the RF port of the mixer. Any variations in the phase of the signal on the RF input caused by a shift in resonant frequency of the LEKID is now measured as a variation in the voltage measured on the I and $\mathrm{Q}$ outputs of the mixer. 


\subsection{Optical set up}

To measure the response of the LEKID to $200 \mu \mathrm{m}$ radiation a blackbody source was installed into the cryostat. The chip carrier lid was modified to contain a $14 \mathrm{~mm}$ aperture closed with a $200 \mu \mathrm{m}$ bandpass filter. The blackbody temperature can be modulated up to around $10 \mathrm{~Hz}$ allowing us to measure the response of the LEKID to a chopped optical source. The details of the optical source are explained in the next chapter. Figure 8.6 shows a schematic of the blackbody source and the modified chip carrier containing the bandpass filter.

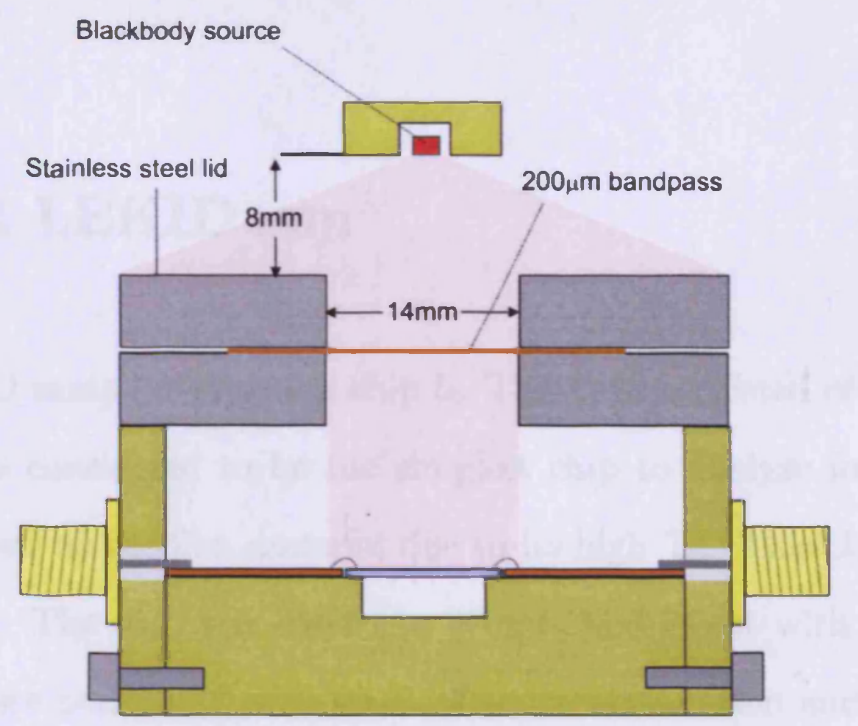

Figure 8.6: The LEKID chip carrier in an arrangement for optical testing. This figure shows a simplified illumination pattern of the blackbody source through the bandpass filter. The optical model is discussed in full in the next chapter. 


\section{Chapter 9}

\section{Experimental data}

\subsection{First LEKID run}

The first LEKID sample tested was chip 5 . This chip consisted of only 6 LEKID devices and was considered to be the simplest chip to analyse for the first run. Niobium was used as the film material due to its high Tc. This chip was labelled "LEKID_1_Nb". The chip was cooled to $300 \mathrm{mK}$ and swept with the VNA. The VNA sweeps were performed with $40 \mathrm{~dB}$ of warm attenuation and found five out of the six resonators on the chip. The details of the chip can be found in table 9.1

Table 9.1: Chip 5 Design values and measured $f_{0}$

\begin{tabular}{ccccc}
\hline \hline LEKID & Coupling distance $(\mu m)$ & $\mathrm{M}(\mathrm{pH})$ & Designed $f_{0}(\mathrm{GHz})$ & Measured $f_{0}(\mathrm{GHz})$ \\
\hline 1 & 150 & 17.3 & 8.474 & $? ?$ \\
2 & 200 & 14.3 & 8.016 & 8.07 \\
3 & 250 & 11.9 & 7.616 & 7.61 \\
4 & 300 & 10.0 & 7.276 & 7.37 \\
5 & 350 & 8.48 & 6.970 & 7.02 \\
6 & 400 & 7.20 & 6.705 & 6.74
\end{tabular}


Each of these resonators had a resonant frequency within about $50 \mathrm{MHz}$ of its design value. These resonators however were very strongly coupled which made further analysis difficult. The weakest coupled resonator (LEKID design 6) could be analysed and showed a $Q_{u}$ of $1.1 \times 10^{6}$ and $Q_{L}=1.8 \times 10^{5}$. The Unloaded quality factor for this resonator was measured by fitting a modified version of equation 5.3 to the resonance data. The modified version has two added terms to account for the offset due to the overall gain/loss in the system and skewing of the resonant feature which is seen in real data. With the unloaded quality factor known the loaded quality factor, $g$ and $M$ can be calculated from the minima in S21 using equations 4.50 and 4.53. Figure 9.1 shows an example of a VNA sweep and the fit to the data of LEKID 6. 

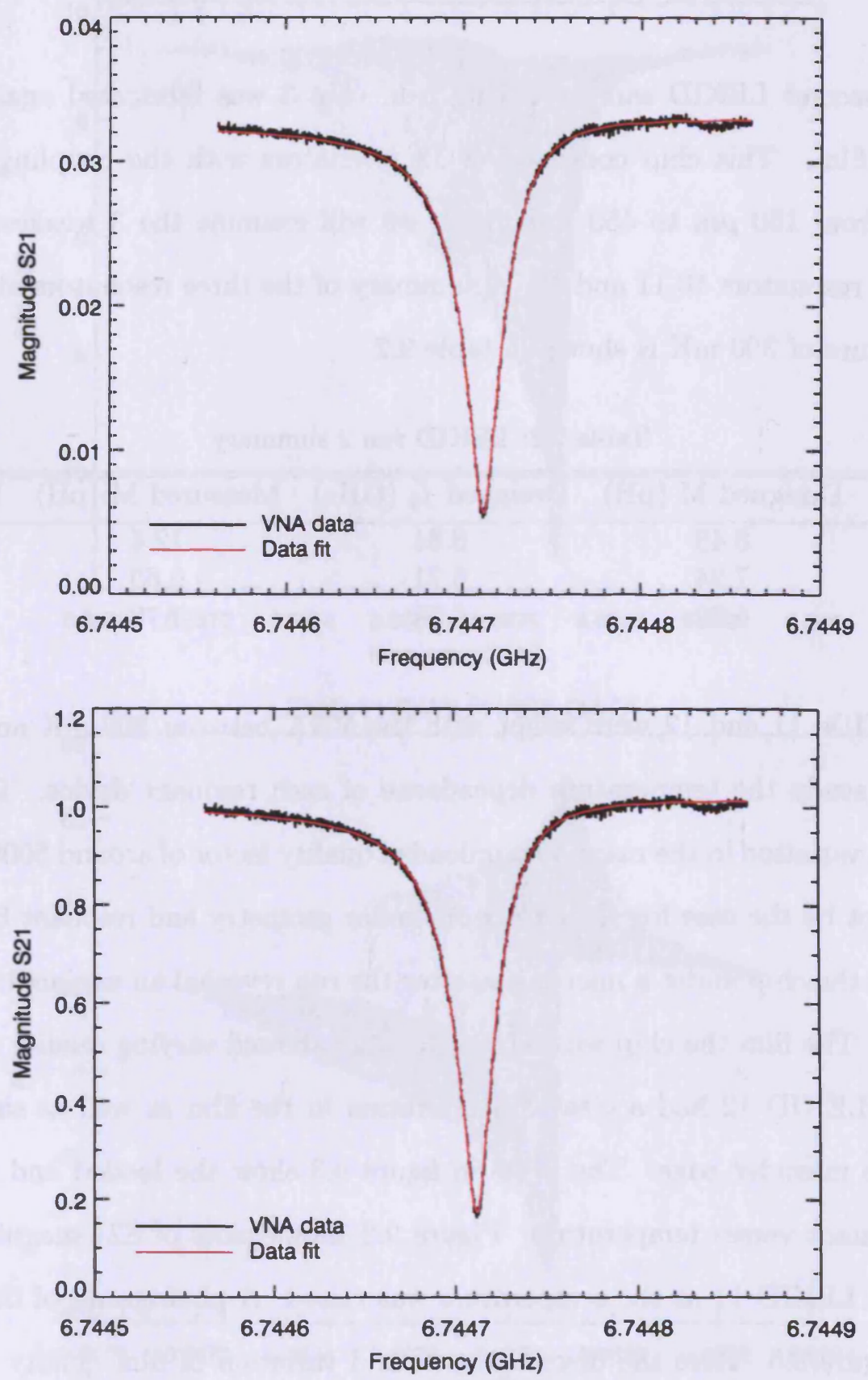

Figure 9.1: (top) LEKID resonance data with fitted curve over-plotted. (bottom) LEKID resonance data with fitted curve over-plotted. Here the offset calculated by the fitting program has been removed. Resonant frequency $=6.744714 \mathrm{GHz}, g=9.93 \pm 0.45 Q_{L}=$ $173000 \pm 7000$ 


\subsection{Second LEKID run}

For the second LEKID sample testing run, chip 3 was fabricated again from a niobium film. This chip consisted of 12 resonators with the coupling distance varying from $150 \mu \mathrm{m}$ to $450 \mu \mathrm{m}$. Here we will examine the 3 weakest coupled LEKIDs, resonators 10,11 and 12. A summary of the three resonators at the base temperature of $300 \mathrm{mK}$ is shown in table 9.2

Table 9.2: LEKID run 2 summary

\begin{tabular}{ccccc}
\hline \hline LEKID & Designed M $(\mathrm{pH})$ & Designed $f_{0}(\mathrm{GHz})$ & Measured M $(\mathrm{pH})$ & Measured $f_{0}(\mathrm{GHz})$ \\
\hline 10 & 8.48 & 6.84 & 12.4 & 6.84 \\
11 & 7.24 & 6.71 & 9.60 & 6.68 \\
12 & 6.22 & 6.58 & 7.78 & 6.56
\end{tabular}

LEKIDs 11 and 12 were swept with the VNA between $300 \mathrm{mK}$ and $2 \mathrm{~K}$ in order to study the temperature dependance of each resonant device. The result showed a variation in the maximum unloaded quality factor of around 500000 . This should not be the case for resonators of similar geometry and resonant frequency. Studying the chip under a microscope after the run revealed an explanation to the problem. The film the chip was fabricated from showed varying quality across its surface. LEKID 12 had a clear discolouration in the film as well as small holes along the meander edge. The plots in figure 9.3 show the loaded and unloaded quality factor verses temperature. Figure 9.2 shows plots of S21 magnitude and phase for LEKID 11 as the temperature was raised. A photograph of this chip is shown figure 9.5. Here the discolouration and variation of film quality is clearly visible around LEKID 12 

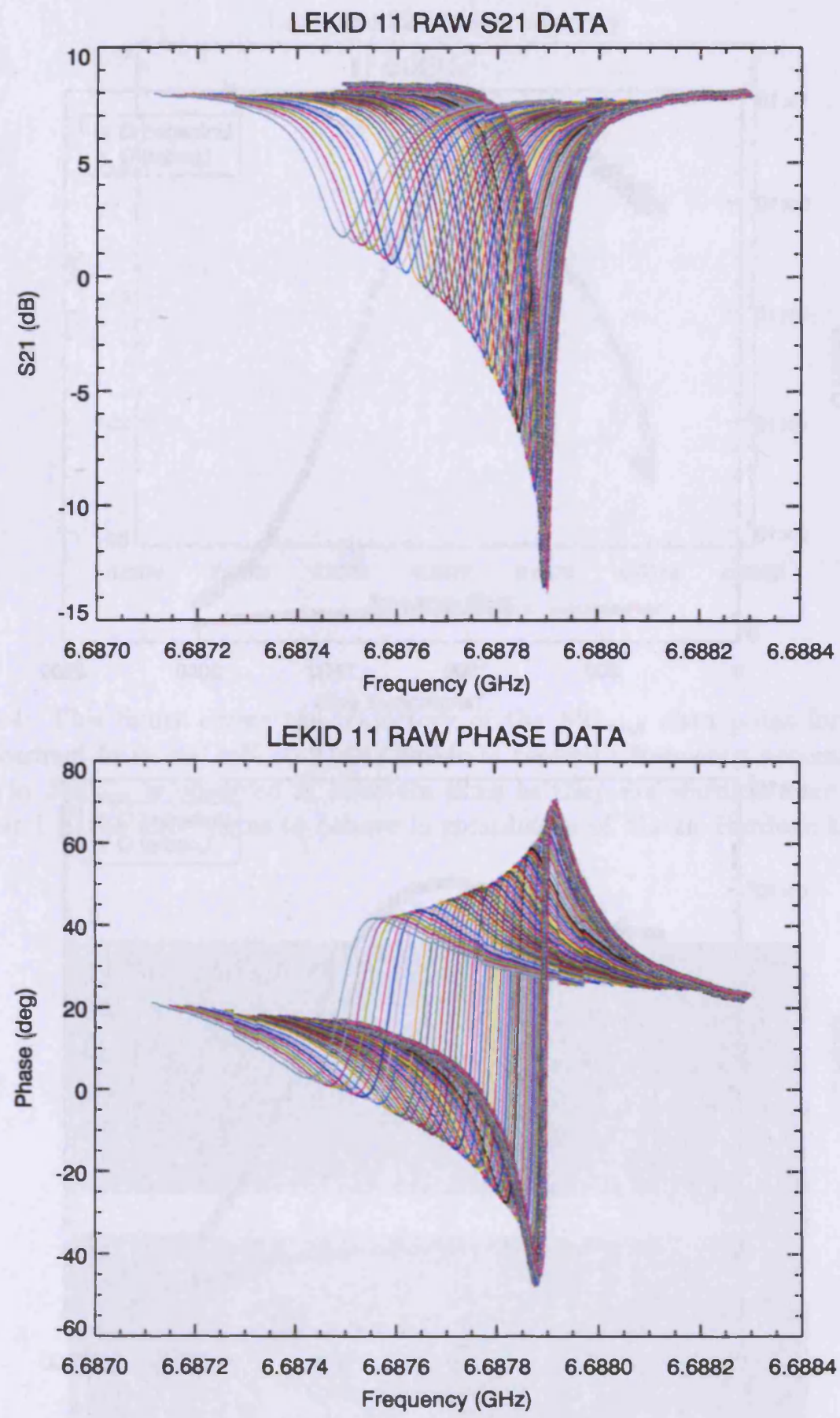

Figure 9.2: The two plots in this figure show the transmission response of warming LEKID 11 from $300 \mathrm{mK}$ (far right curve in each plot) to $2.0 \mathrm{~K}$ (far left curve in each plot). (top) Raw S21 magnitude data for LEKID 11. (bottom) Raw S21 phase data for LEKID 11. 

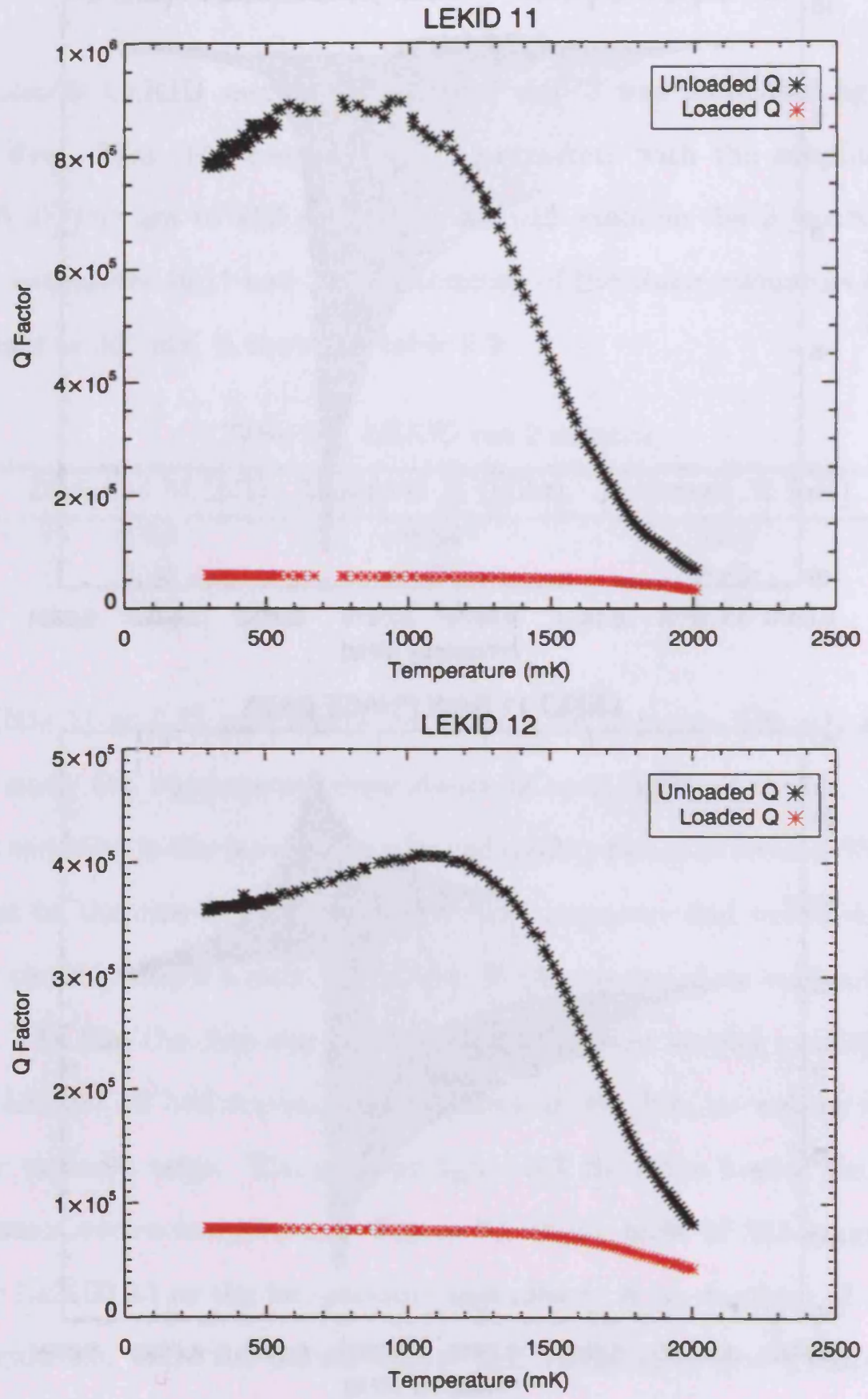

Figure 9.3: $Q_{u}$ and $Q_{L}$ vs temperature for LEKIDs 11 and 12. Notice in both plots a rise in $Q_{u}$ is observed before it begins to fall. This effect is not described by Mattis-Bardeen theory. 


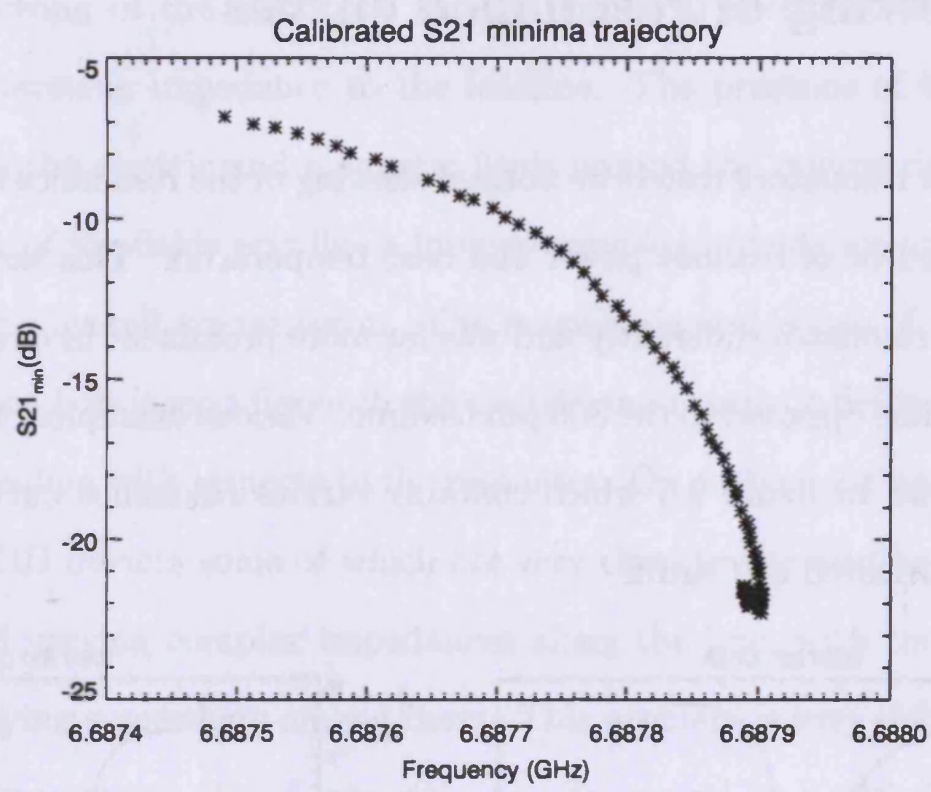

Figure 9.4: This figure shows the trajectory of the $S 21_{\min }$ data point for LEKID 11 as it is warmed from $300 \mathrm{mK}$ to $2.0 \mathrm{~K}$. A rise in resonant frequency accompanied by a decrease in $S 21_{\min }$ is observed in Niobium films as they are warmed from $300 \mathrm{mK}$ to $1 \mathrm{~K}$. After $1 \mathrm{~K}$ the film begins to behave in compliance of Mattis-Bardeen theory.

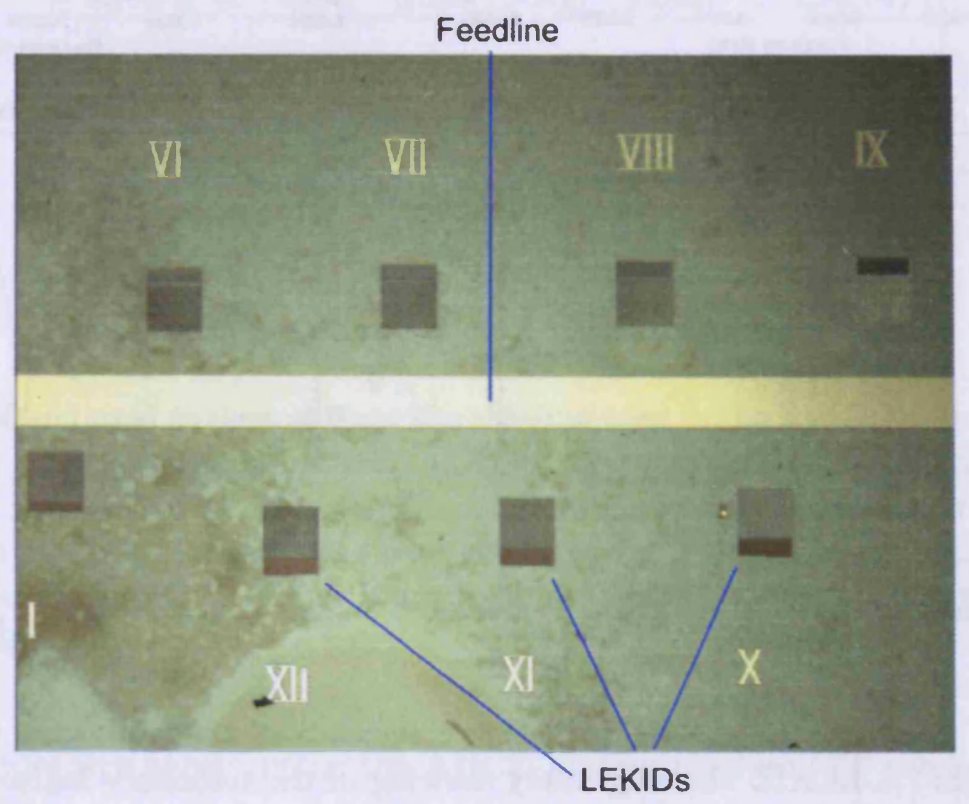

Figure 9.5: Photo of chip LEKID2 


\subsection{Skewing of resonance curves}

On each of the resonators tested we noticed skewing of the resonance feature which seems independent of readout power and base temperature. This skewing seemed to affect each resonator differently and was far more prominent in devices fed from a $100 \mu \mathrm{m}$ feedline opposed to the $300 \mu \mathrm{m}$ feedline. Various examples of this skewing effect are shown in figure 9.6 which contains various resonance curves measured from chips fabricated at Cardiff.
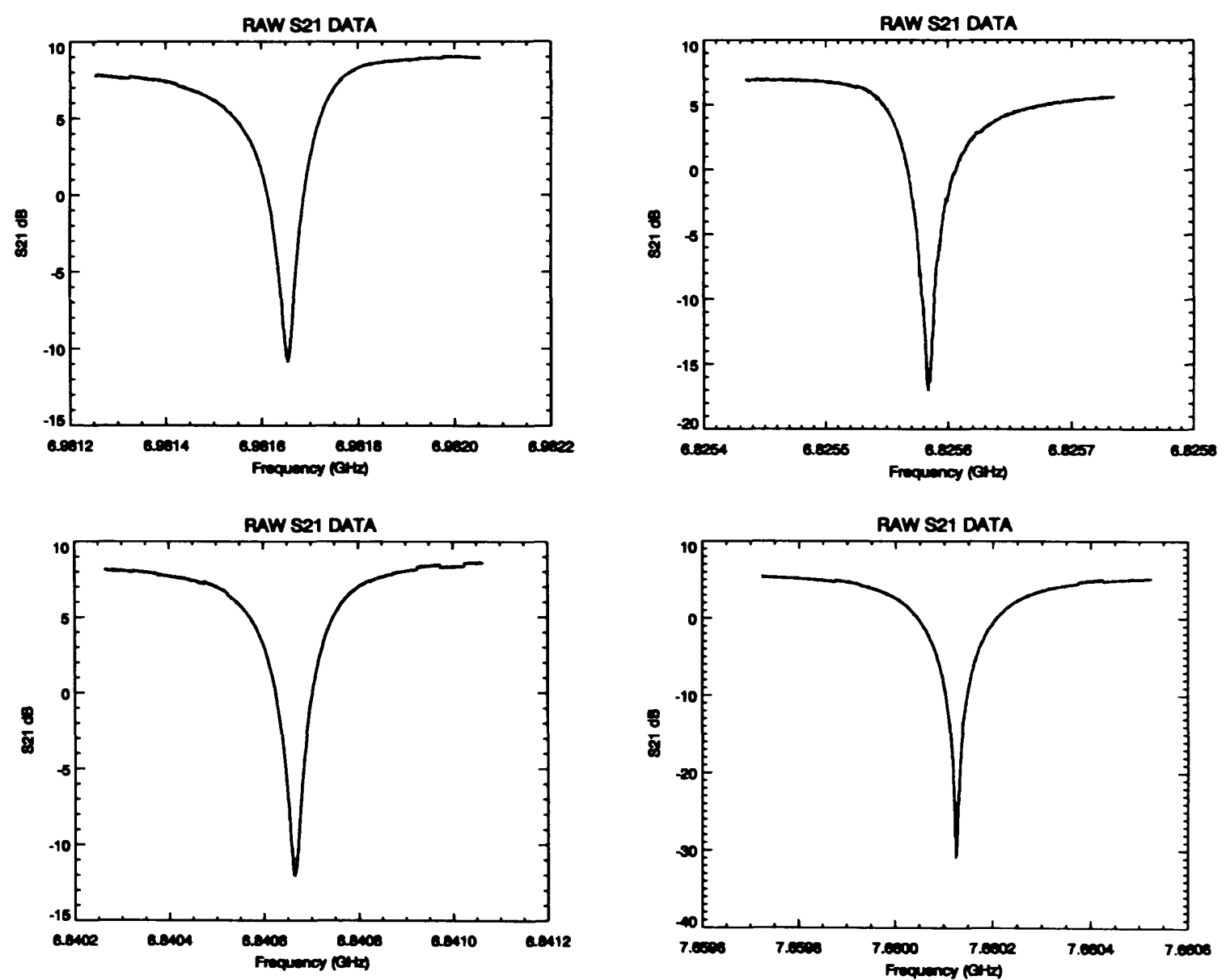

Figure 9.6: (top left) A LEKID with heavy skewing of the resonance feature to the left. (top right) A LEKID showing heavy skewing of the resonance feature to the right. (bottom left) A LEKID with slight skewing to the left. (bottom right) A LEKID with almost no skewing. 
The skewing of the resonance feature is due to each resonator introducing a complex parasitic impedance to the feedline. The presence of the LEKID will modify both the electric and magnetic fields around the microstrip feedline. The modification of the fields acts like a lumped complex impedance on the line which will affect the overall transmission. The magnitude and phase of this impedance will depend on how it modifies both the electric and magnetic fields and its position along the feedline with respects to the resonator. On each of our test chips we have several LEKID devices some of which are very close to the feedline. This leads to a cascade of varying complex impedances along the line, with the LEKID being measured laying somewhere among them. This problem is very difficult to analyse due to the uncertainty of each parasitic impedance and its position relative to the resonator being measured, however we can demonstrate this effect using the model shown in figure 9.7. Here we place the LEKID at a set position on the feedline and vary the position of a $1 \mathrm{nH}$ lumped inductance to demonstrate the skewing effect.

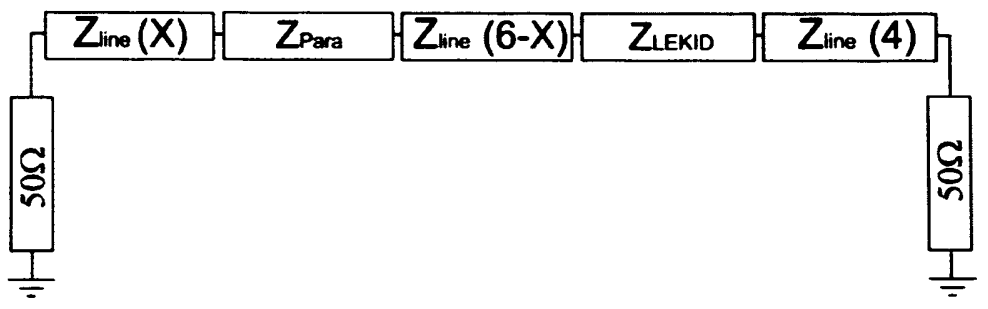

Figure 9.7: Model used to demonstrate the skewing seen in the LEKID resonance feature. Here $Z_{\text {line }}(X)$ is a $50 \Omega$ line of length $X \mathrm{~mm}, Z_{\text {Para }}$ is a $1 \mathrm{nH}$ parasitic inductance, $Z_{\text {line }}(6-X)$ is a second $50 \Omega$ variable length of line to set the resonator at a distance of $6 \mathrm{~mm}$ from the beginning of the first line, $Z_{L E K I D}$ is the effective impedance of the LEKID resonator and $Z_{\text {line }}(4)$ is a $4 \mathrm{~mm}$ length of $50 \Omega$ line leaving the total length of the system $10 \mathrm{~mm}$

This model was solved using the ABCD matrix method [40] used earlier in this report. Figure 9.8 shows four results obtained by a resonator with a given $Q_{u}, f_{0}$ and $M$ influenced by a $1 \mathrm{nH}$ parasitic inductance placed at various positions along 

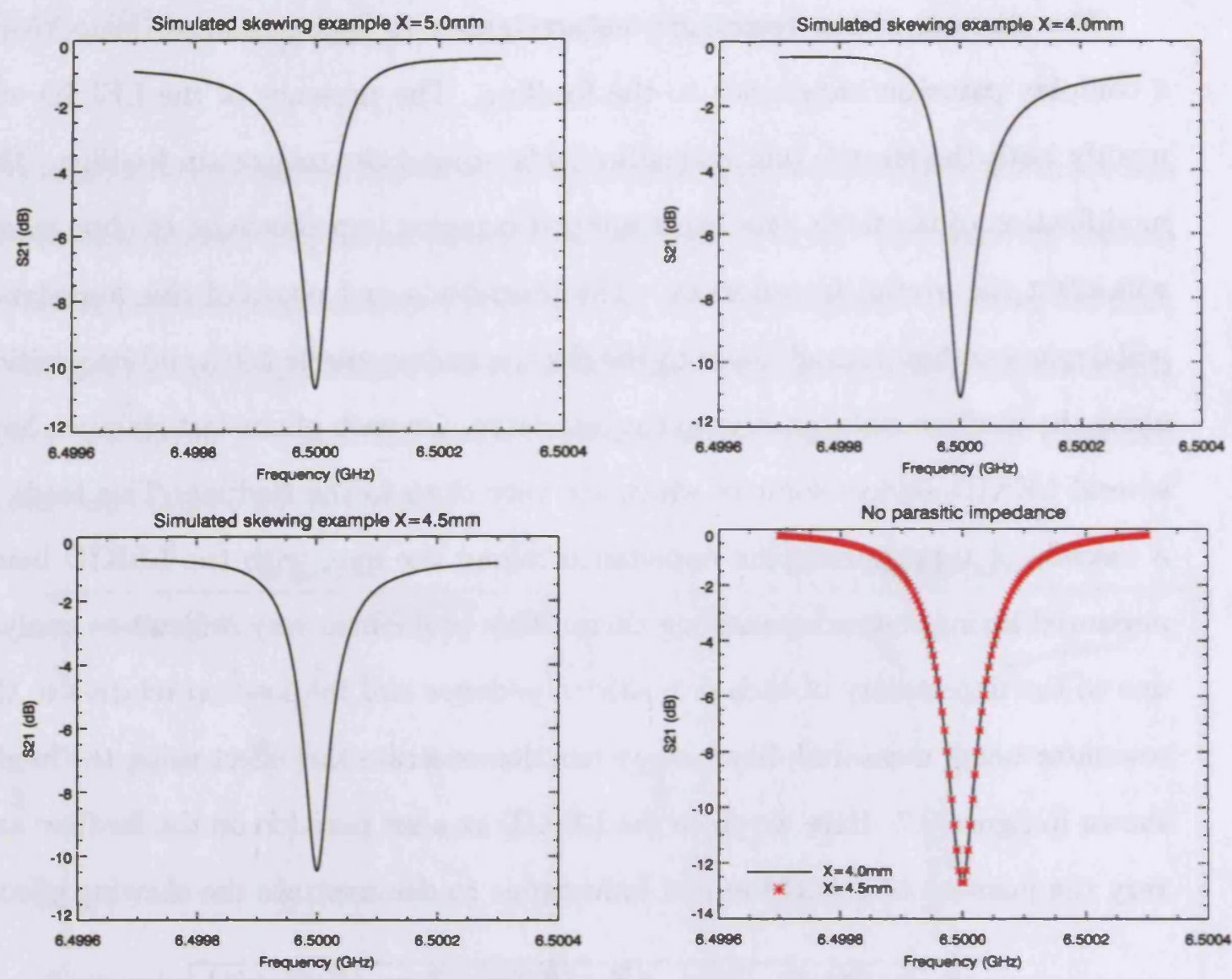

Figure 9.8: (top left) Simulation to demonstrate a resonance feature skewed to the left. (top right) Simulation demonstrating skewing to the right. (bottom left) A simulation showing that at a certain distance from the resonator the parasitic impedance leaves the resonance feature un-skewed. (bottom right) A LEKID at two positions (X) along a $10 \mathrm{~mm}$ feedline. Here we see that the position of the LEKID along a matched parasitic free line has no influence on the resonance feature.

the $6 \mathrm{~mm}$ length of feed line that proceeds it. The last plot, (bottom right) shows the effect of moving the LEKID along the line with no parasitic impedance added. This last result shows that in the absence of parasitic impedances the resonant feature is independent of position along the feedline. The plots also demonstrate how the parasitic impedances can alter the coupling of the LEKID to the feedline. This explains some of the error we see in the measured values of $M$ we see in real LEKID devices. 


\subsection{Back-bending}

In all the niobium resonators we measured at Cardiff we see the resonance feature evolve in the opposite way to which it should with rising temperature for temperatures below $T \approx 1 \mathrm{~K}$. We have observed this effect in niobium distributed resonators as well as lumped element resonators. This effect has also been observed in tantalum and aluminium distributed KIDs at very low temperature by the SRON group [72]. This phenomena has been dubbed "back-bending". The physical process behind this effect are not well understood and is not described by Mattis-Bardeen theory. The most likely explanation is a change in dielectric constant of either the substrate or an oxide layer on the film with temperature which outweighs the change in kinetic inductance at very low temperature. If this effect is indeed due to a change in dielectric constant then its effect should not be seen under photon absorption. Under photon absorption, the lattice of the film, substrate and oxide layer all remain at the same temperature with only the electron temperature rising, hence the shift in resonant frequency and phase should evolve as predicted by Mattis-Bardeen theory. This theory needs to be confirmed by a series of optical experiments with various film in which back-bending has been observed.

\subsection{Optical testing}

Once we were confident that we could demonstrate the LEKID as a superconducting micro-resonator a test was devised to try and detect $200 \mu \mathrm{m}$ radiation from a chopped blackbody source. The optical set up described in the last section was used in conjunction with Chip 3 fabricated from an aluminium film. The 
aluminium for this film was thermally evaporated onto a $325 \mu \mathrm{m}$ sapphire substrate using an E-beam system. All the resonators on this film were limited by the unloaded quality factor of the film at $280 \mathrm{mK}\left(Q_{u} \approx 17000-20000\right)$. The film thickness was $130 \mathrm{~nm}$, and so the measured $Q_{u}$ for this LEKID geometry is in accordance to the predicted $Q_{u}$ from the plot in figure 6.5. However in order to try and increase the $Q_{u}$ of this resonator the cryostat was pumped on. This action reduced the temperature of the $4.2 \mathrm{~K}$ base plate to around $1.8 \mathrm{~K}$ which in turn led to the sample stage temperature reducing to $250 \mathrm{mK}$. This had no effect on the measured $Q_{u}$ of the LEKID indicating that the film was of poor quality most likely due to amorphous growth during the evaporation process or a high impurity content.

LEKID 5 was chosen to be used for the optical testing as it showed the highest loaded quality factor $\left(Q_{L} \approx 1.0 \times 10^{4}\right)$ and only small amounts of skewing of the resonance feature. For the optical testing a precision signal generator (PSG) was borrowed from the Cardiff school of Electrical Engineering. This source is far cleaner than the VNA in CW mode and can deliver more power which is needed to drive the local oscillator input of the IQ mixer. The blackbody source was courtesy of Dr Peter Hargrave. This source was used for calibration experiments of the SPIRE instrument [73] and was part of a number of other optical components when calibrated. Our optical set up had only consisted the source without the optics and for this reason a calibrated blackbody power was not available. However, we were able to make estimates of the radiated power of the bare source from estimates of its temperature and apply the relevant geometrical arguments to estimate the power incident on the LEKID pixel. The lid of the LEKID sample holder had a $14 \mathrm{~mm}$ circular aperture closed with a $200 \mu \mathrm{m}$ metal mesh bandpass filter [74] to define the optical band. The band pass filter was provided by Professor Peter Ade 
and Dr Carole Tucker. The bandpass characteristics of this filter were measured on an Fourier Transform Spectrometer (FTS) by the Cardiff filter group. The transmission of this filter can be seen in figure 9.9 .

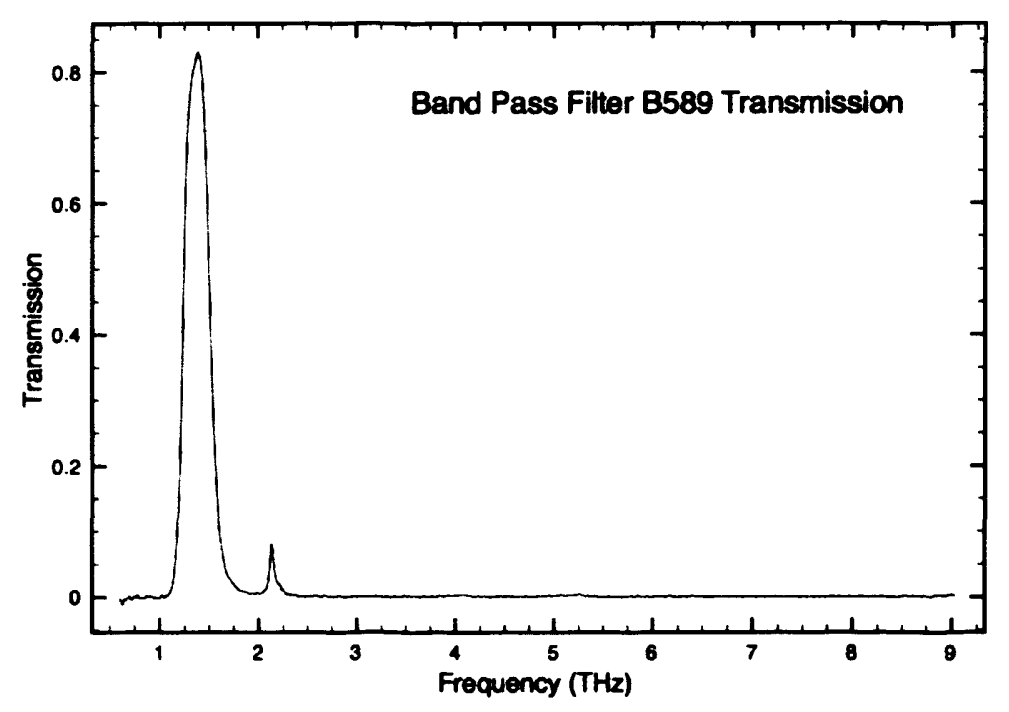

Figure 9.9: Optical transmission of the $200 \mu m$ band pass filter (filter reference B589)

To estimate the blackbody power incident on the LEKID pixel the following assumptions were made about the system shown in figure 8.6 :

- The source is a perfect blackbody.

- The blackbody source is $1 \mathrm{~mm}$ square and due to the mounting structure all the power from both the front and back surfaces is radiated towards the detector (The power from the backside first being reflected off the mounting structure). This gives an effective surface area of $2 \mathrm{~mm}^{2}$

- At the distance of the aperture $(8 \mathrm{~mm})$ the source can be approximated as a point source at a given temperature radiated in to $2 \pi s t r$

- All the light incident on the aperture (area $\pi R^{2}$ ) is radiated as a collimated beam with perfect efficiency. Here we are assuming the aperture in the lid is acting as a perfect light-pipe due to its length. 
- The collimated beam illuminates the chip surface evenly. That is to say the beam does not diffract and creates a homogeneous spot of diameter $14 \mathrm{~mm}$ across the chip surface.

From an optics point of view these assumptions are very crude, however, the assumptions made here will give an over estimate of the flux density at the surface of the chip, and taking into account that we can at best only estimate the temperature of the blackbody source this model will suffice. The power incident on the LEKID pixel can be calculated by integrating the blackbody curve for a blackbody of area (A) and temperature (T) multiplied by the filter bandpass transmission curve. The optical power $\left(P_{\text {opt }}\right)$ radiated through the bandpass filter $(\mathrm{BP}(\nu))$ of a blackbody with temperature $\left(T_{B B}\right)$ and surface area $\left(A_{B B}\right)$ is given by [75]:

$$
P_{o p t}=A_{B B} \int_{0}^{\infty} \frac{2 h \nu^{3}}{c^{2}} \frac{1}{\exp \left(h \nu / k_{B} T_{B B}\right)-1} B P(\nu) d \nu
$$

Using the assumption that the blackbody is far enough away from the aperture that we can regard it as a point source and that all its power is radiated into $2 \pi$ steradians the power radiated through the aperture is:

$$
\gamma=\frac{P_{o p t}}{r^{2}} A_{a p}
$$

Here $A_{a p}$ is the cross sectional area of the aperture and $r$ is the distance of the aperture to the source. The power radiated on to each LEKID pixel can then be calculated from the ratio of area of the aperture and the LEKID pixel. Table 9.3 gives the expected power radiated on to each LEKID pixel for a blackbody source of area $2 \mathrm{~mm}^{2}$ at a distance of $8 \mathrm{~mm}$ from an aperture of radius $7 \mathrm{~mm}$. 
Table 9.3: Radiated blackbody power

\begin{tabular}{lccc}
\hline \hline$T_{B B}$ & $30 \mathrm{~K}$ & $35 \mathrm{~K}$ & $40 \mathrm{~K}$ \\
\hline$P_{\text {opt }}(\mathrm{pW})$ & 2414 & 3512 & 4725 \\
$P_{L E K I D}(\mathrm{pW})$ & 2.35 & 3.43 & 4.61
\end{tabular}

The blackbody source was driven by a $4 \mathrm{~V}$ peak to peak sine wave at $4 \mathrm{~Hz}$ with zero offset. The blackbody is simply a resistive load and so power should be delivered to it in both the positive and negative half of the cycle, hence the modulation frequency of the blackbody will be $8 \mathrm{~Hz}$. The resonance frequency of LEKID 5 was $7.67 \mathrm{GHz}$ and was found by sweeping with the VNA. The PSG was then set to this resonance frequency. The I and $\mathrm{Q}$ channels were sent directly to the Textronics scope to be measured. Data were collected by sampling the I and $\mathrm{Q}$ channels at $1 \mathrm{KHz}$ for 20 seconds. The data were then transferred from the scope into Interactive Data Language (IDL) [76] for analysis. The I and Q offsets were calculated by measuring both I and Q with the oscilloscope while the LEKID was on resonance. Noting that the phase on resonance should be zero, the data sets collected could be rotated in the IQ plane accordingly to measure a phase of zero degrees on resonance. The figures 9.10, 9.11 and 9.12 show the raw and filtered I and $\mathrm{Q}$ time steams.

The phase is measured by taking the inverse tangent of $I / Q$. The plots in figures 9.13 and 9.14 show the phase response of LEKID 5 .

Taking the Fourier transform of the raw phase time stream gives us the noise spectrum for the device. The noise spectrum is shown in figure 9.15. This plot clearly shows our signal at $8 \mathrm{~Hz}$ and the large amounts of $1 / \mathrm{f}$ noise which can also be seen in the filtered time streams. Over-plotted in the plot of figure 9.15 is the off resonance noise spectrum. Here the blackbody was still being driven by a $4 \mathrm{~Hz}$, $4 \mathrm{Vpp}$ sine wave. This plot confirms that we are observing the LEKID response as 


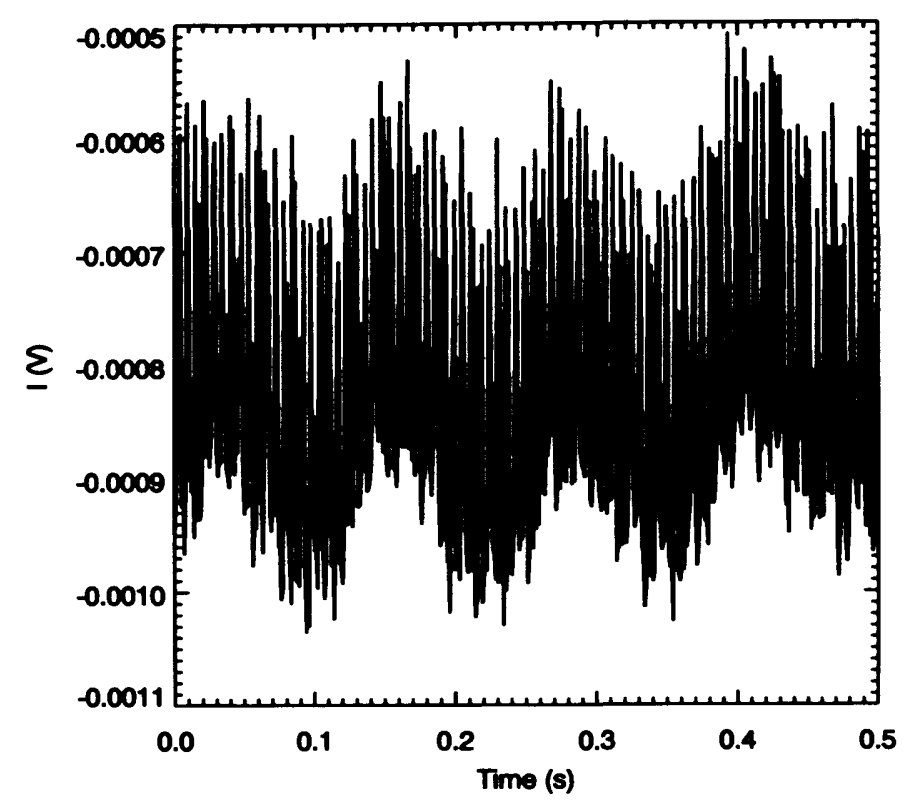

Figure 9.10: Raw I time stream for LEKID 5 illuminated with a blackbody modulated at $8 \mathrm{~Hz}$

we see no $8 \mathrm{~Hz}$ signal as we move from resonance.

We measure a signal to noise ratio of 75 from the plot in figure 9.15. This is done by measuring the peak of the $8 \mathrm{~Hz}$ signal divided by the noise value at $8 \mathrm{~Hz}$ when the blackbody source is turned off. Using this ratio we can estimate an optical NEP for this detector from estimating the power incident on it. Table 9.4 quotes the estimates of NEPs from the incident power estimates of table 9.3.

Table 9.4: Estimated Optical Noise Equivalent Powers

\begin{tabular}{lccc}
\hline \hline$T_{B B}$ & $30 \mathrm{~K}$ & $35 \mathrm{~K}$ & $40 \mathrm{~K}$ \\
\hline $\mathrm{NEP}(W / \sqrt{H z})$ & $3.13 \times 10^{-14}$ & $4.5 \times 10^{-14}$ & $6.14 \times 10^{-14}$
\end{tabular}

After obtaining the various plots for detected signal we wanted to rule out the possibility that our signal was from electrical pickup opposed to real optical detection. Electrical pickup was ruled out by the following points: 


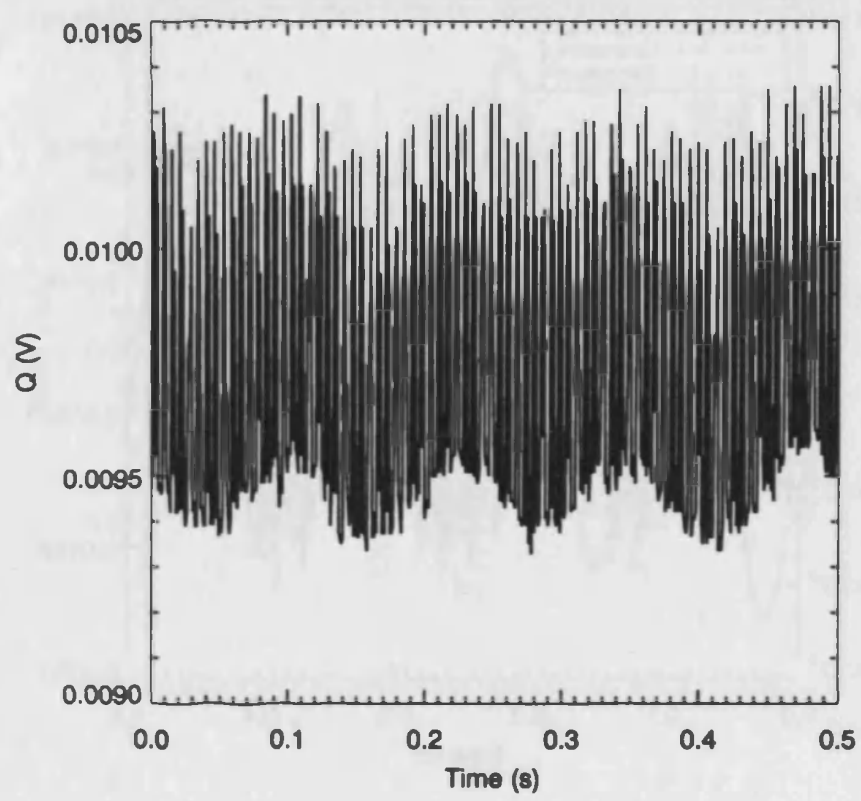

Figure 9.11: Raw $Q$ time stream for LEKID 5 illuminated with a blackbody modulated at $8 \mathrm{~Hz}$

- The drive for the blackbody source is completely isolated from the rest of the cryostat. This minimises the possibility of ground loops existing within the set up.

- The blackbody was driven by a $4 \mathrm{~Hz}$ voltage source. Electrical pickup would be expected at $4 \mathrm{~Hz}$, instead we measure our signal at $8 \mathrm{~Hz}$ which is equal to the modulation frequency of the blackbody.

- We see no signal off resonance. The plot in figure 9.15 clearly shows that we measure no signal off resonance despite the fact that the blackbody was still modulated by the same $4 \mathrm{Vpp} 8 \mathrm{~Hz}$ drive.

- The voltage measured on the I and Q channels are 180 degrees out of phase: this can be seen in the plot in figure 9.12. This indicates that the phase of the RF port of the IQ mixer is changing with respects to the LO. If the signal was due to pickup (through the parasitic modulation of an amplifier bias for 


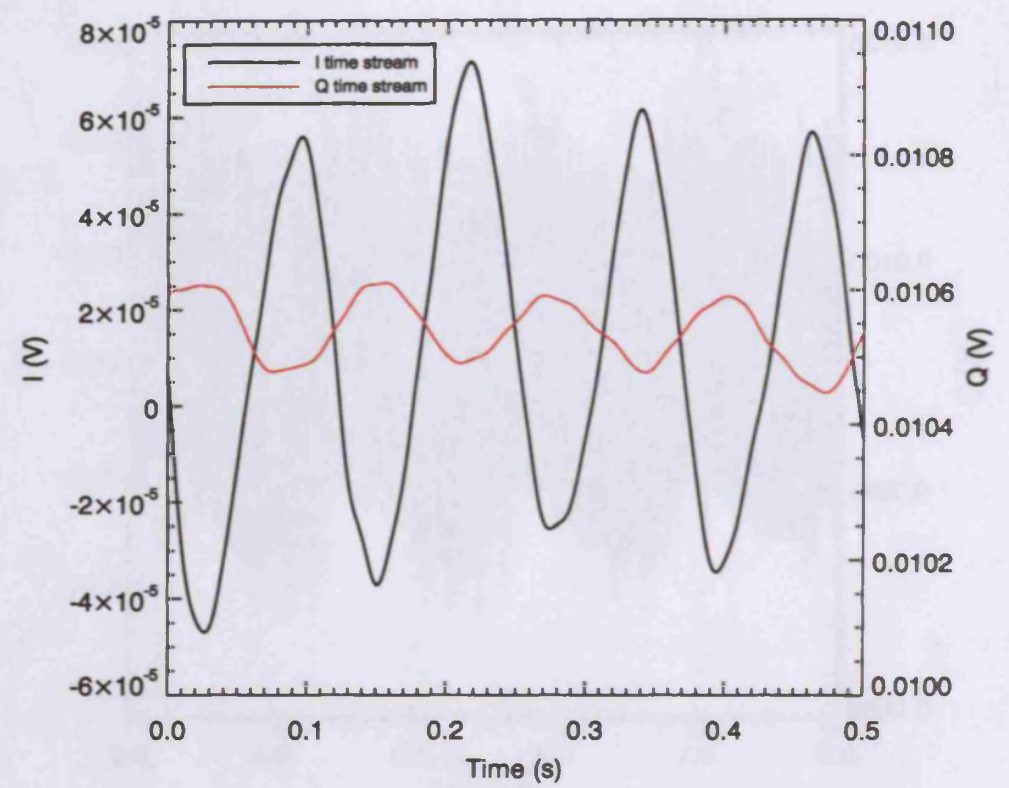

Figure 9.12: Filtered I and Q time streams for LEKID 5 illuminated with a blackbody modulated at $8 \mathrm{~Hz}$. Here the raw I and $\mathrm{Q}$ time steams have been filtered using a Gaussian filter in Fourier space.

example) we would expect the RF and LO inputs to be in phase and the pickup signal would be due to the changing amplitude of the RF input only.

\subsection{Predicted optical efficiency}

From the simulations performed in chapter 7 we can estimate the optical efficiency of this LEKID device as a detector. The sheet impedance of the film was measured by a DC measurement of the feedline at $4.2 \mathrm{~K}$. The measured sheet resistance was $\approx 0.3 \Omega$ /square, with a $s / w$ ratio of 3 this gives an effective sheet impedance of $Z_{\text {eff }}<1 \Omega$ /square. The substrate was sapphire of thickness $325 \mu \mathrm{m}$ giving a thickness of $\approx 5 \lambda$ at $1.5 \mathrm{THz}$. Figure 9.16 shows the predicted absorption for this device between 1 and $2 \mathrm{THz}$. 


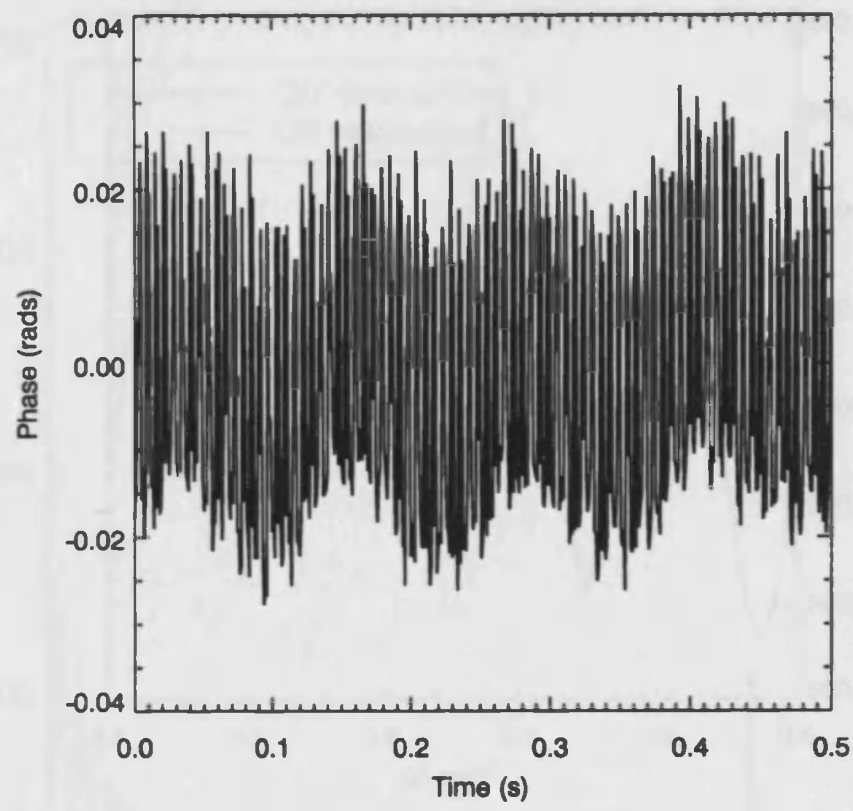

Figure 9.13: Raw phase time stream for LEKID 5 illuminated with a blackbody modulated at $8 \mathrm{~Hz}$.

This chip was designed to test the microwave theory of the LEKID and not designed for high optical efficiency, hence we see less than $2 \%$ optical efficiency across the band. The thick substrate also give rise to fringing effects where we see a dramatic fall in optical efficiency when the substrate thickness is equal to multiples of $\lambda / 2$.

\subsection{Experimental data conclusion}

In this chapter we have proven that the LEKID concept works from a microwave standpoint and have demonstrated optical coupling of $200 \mu \mathrm{m}$ radiation to the LEKID meander. The analysis of the data from the tested devices agrees with that of Mattis-Bardeen theory and the microwave design of the LEKID from both the Sonnet and analytical simulations detailed in chapters 4 and 5 . The test performed 


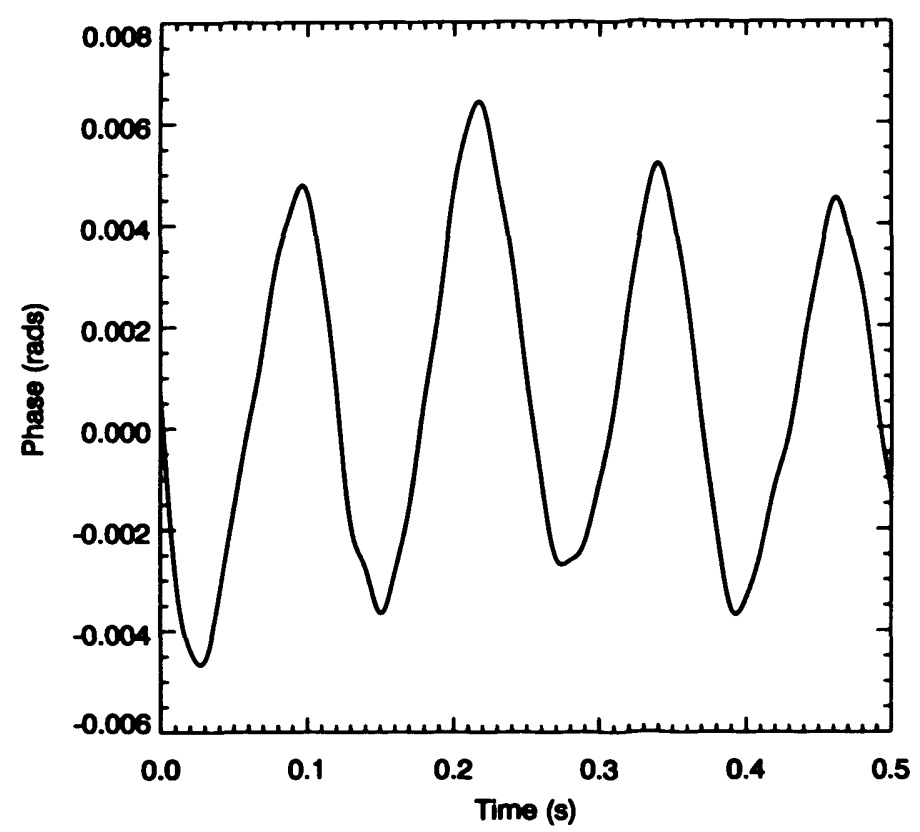

Figure 9.14: Filtered phase time stream for LEKID 5 illuminated with a blackbody modulated at $8 \mathrm{~Hz}$. Here the raw phase time steam has been filtered using a Gaussian filter in Fourier space.

on the various LEKID devices also showed that these devices can be heavily effected by parasitic imedances caused by other LEKID resonators disrupting the fields around the feedline. This effect should not be a problem for future LEKID designs as we also demonstrated that these resonators were over coupled and that in fact they could be moved further from the feedline minimising this parasitic impedance effect while at the same time increasing the $Q_{L}$ and hence responsivity. The optical coupling tests, although crude, are still very encouraging. It is clear from the fact that the unloaded $Q$ was limited to around 20000 that this film was of poor quality. High quality aluminium films have demonstrated $Q_{u}$ values in excess of 450000 at $100 \mathrm{mK}$ [50]. Such material, if used for a LEKID would allow for the design of a reduced mutual inductance between the feedline and the LEKID. This opens up opportunities to couple to feedlines with far greater confinement of the fields such as coplanar wave guide (CPW). A CPW feedline would allow for all the processing 


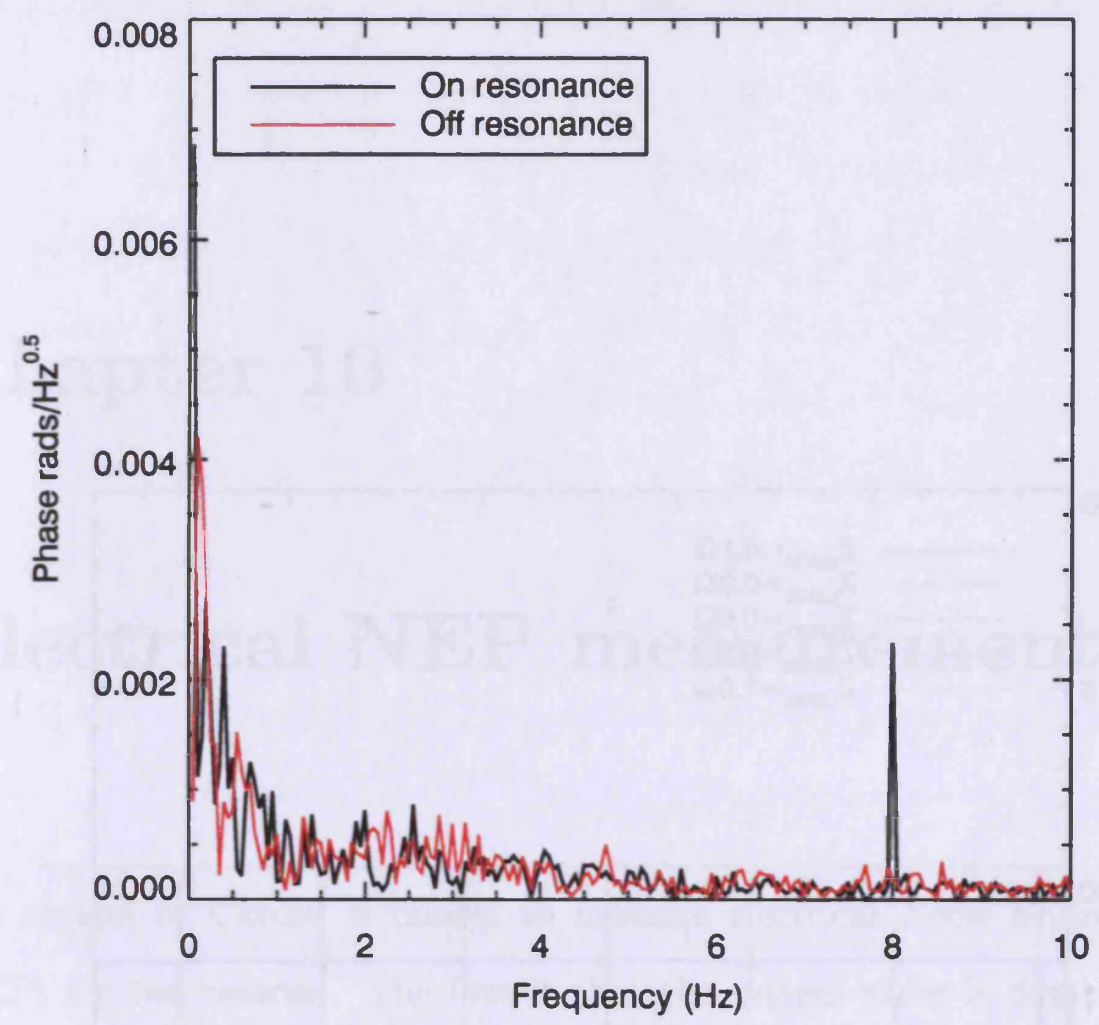

Figure 9.15: The FFT of the phase time streams on and off resonance with the blackbody being driven by a $4 \mathrm{Vpp}, 4 \mathrm{~Hz}$ sine wave.

to be performed on a single layer and would also create a simple solution to back illumination as shown in figure 7.10. 


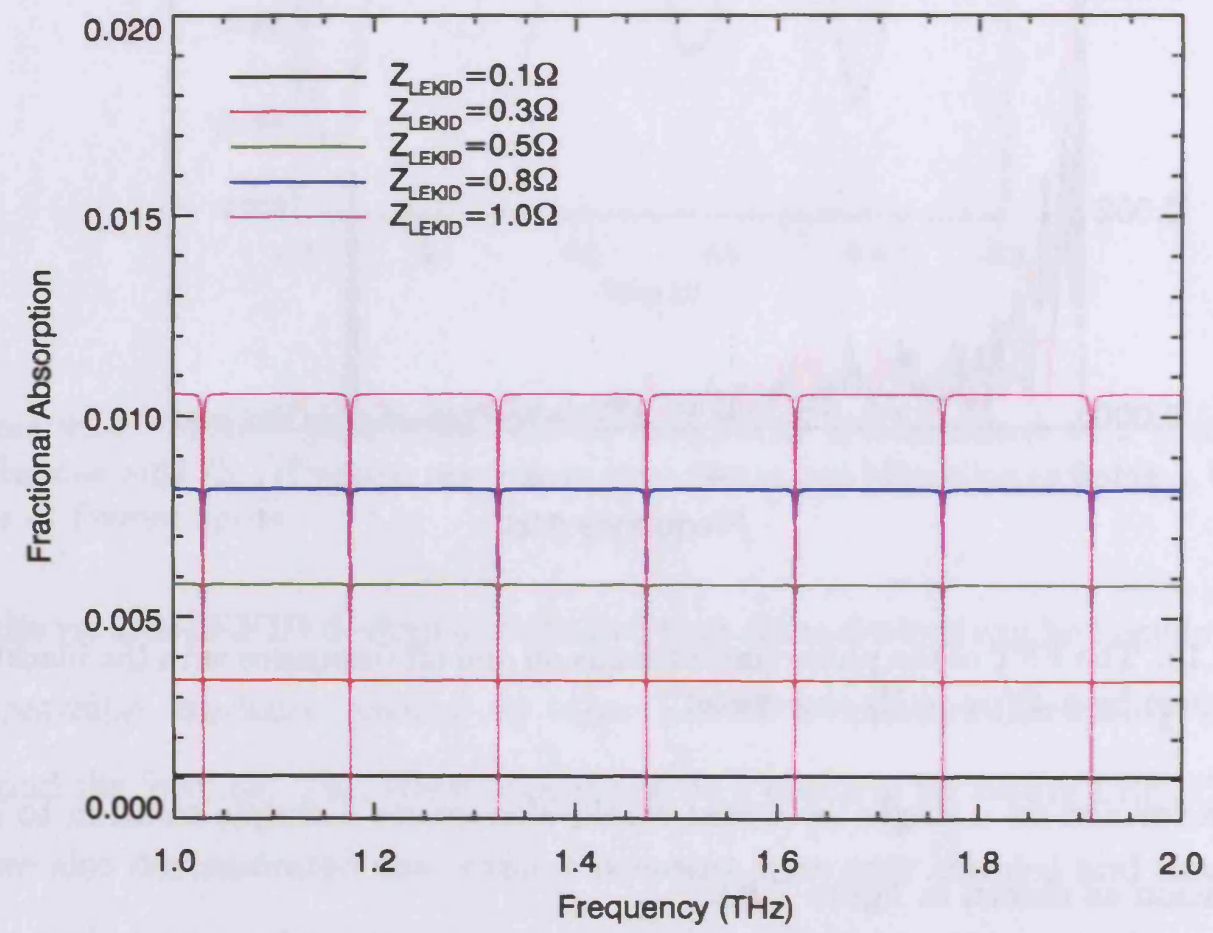

Figure 9.16: Predicted optical efficiency of LEKID 5 using the transmission line model in chapter 7 . Due to the small $s / w$ ratio and high film conductivity this device has a predicted optical efficiency of less than $2 \%$. 


\section{Chapter 10}

\section{Electrical NEP measurements}

The system at Cardiff is unable to measure electrical Noise Equivalent Power (NEP) for two reasons. The first is that the system noise is dominated by the first stage amplifier which is at $300 \mathrm{~K}$ and the second is that we are not set up to measure the quasi-particle lifetime in our films. To measure electrical NEP the following steps must be taken.

- The response of the KID device must be measured. This is done by measuring $\mathrm{I}$ and $\mathrm{Q}$ while sweeping the microwave probe signal across the resonance feature at varying temperatures. By noting the resonant frequency at the base temperature the evolution of both the amplitude and phase can be measured as a function of temperature. Using equation 3.40 the change in temperature can be translated into the change in the number of quasiparticles in the resonator. From this calculation we can effectively measure the change in amplitude and phase of our resonator per quasi-particle created.

- The quasi-particle lifetime must be measured. The change in the number 
of quasi-particles in the resonator will depend on two factors, the number of quasi-particles created per unit time and the quasi-particle lifetime. The number of quasi-particles created per unit time will depend on the optical power absorbed by the detector, whereas the quasi-particle lifetime is determined by the material properties and the temperature of the superconducting film. As temperature is increased the quasi-particle lifetime decreases, dictated by equation 3.41. The quasi-particle lifetime is akin to the weak thermal link to the heatsink in a standard bolometer and, like in a standard bolometer, will determine the time time constant of the KID device. The quasi-particle lifetime is measured using an optical pulse from an LED. The pulse will break a large number of pairs and perturb the KID device from resonance. The quasi-particles will recombine as Cooper pairs at a rate determined by their lifetime until the quasi-particle equilibrium state has been reached. By measuring both $I$ and $Q$ throughout this process, the time taken for the KID device to return to its resonant frequency can be measured and hence the quasi-particle lifetime can be obtained.

- The phase and amplitude noise must be measured. The optical response of a KID device can be measured by looking at the change in amplitude or phase of the probe signal as the resonant frequency shifts. As demonstrated earlier the phase response is far greater than the amplitude response in KID devices and for this reason would be easier to detect above the system noise. However excess phase noise generated by a KID device $[3,77]$ has made measuring the amplitude response more desirable [50].

In order to measure an electrical NEP for our LEKID devices we took sample LEKID_5_Al to the Space Research Institute of the Netherlands (SRON). The sensor research and technology group at SRON have developed a fully calibrated 
testbed capable of making full electrical NEP measurements of several KID devices on a single chip at temperatures as low as $50 \mathrm{mK}$. The excellent facility was developed by Dr Jochem Baselmans and Dr Stephen Yates, who both kindly assisted in this stage of the LEKID development.

\subsection{SRON test set up}

The testbed at SRON has been developed around a Vericold pulse tube ADR capable of holding a base temperature of $50 \mathrm{mK}$ in excess of two days. The cold $\mathrm{RF}$ chain is similar to the Cardiff set up but has a few additional features. These are namely a cryogenic low noise amplifier and two superconducting low-pass filters. A schematic of the SRON cold RF chain is shown in figure 10.1

The two superconducting low-pass filters serve two purposes. The first is that they aid thermalisation of the centre conductor of the coaxial lines connecting to the sample as these devices are planar structures. The second is they filter out any unwanted high frequency signals generated in the warmer parts of the system as thermal noise. The cold amplifier is used to boost the heavily attenuated probe signal before it is attenuated further by the very lossy cables used within the cryostat. These amplifiers have noise temperatures as low as $T_{e}<8 K \approx$ (noise figure $\approx 0.11 d B$ ) and so out-perform the first stage amplifier in the Cardiff set up by around a factor of 8 . 


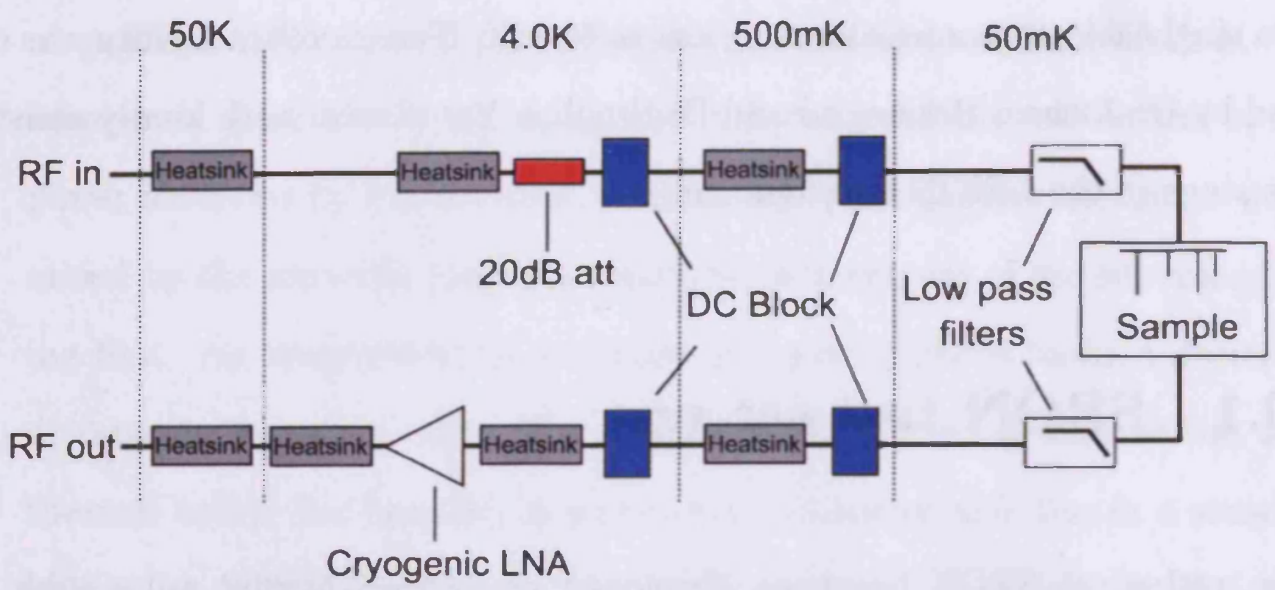

Figure 10.1: The SRON cold RF chain. This RF chain is similar to the Cardiff cold RF chain but has the additional features of two low-pass filters on the $50 \mathrm{mK}$ stage and a cryogenic low noise amplifier mounted on the $4 \mathrm{~K}$ stage. The coaxial cables connecting the $4 \mathrm{~K}$ stage to the $50 \mathrm{mK}$ stage are made from Niobium. Niobium coaxial cable has a great advantage over the Cupronickel coaxial cables used in the Cardiff set up, that is as well as providing thermal isolation these cables will be superconducting. This reduces unwanted losses after the sample has been probed and effectively lowers the noise temperature of the system.

\subsection{Responsivity measurements}

As mentioned earlier, the responsivity of a KID device is calculated by measuring the IQ response of a KID being swept across its resonance feature at various temperatures and the translating this to a variation in amplitude and phase per quasi-particle broken. In order to do this accurately a full calibration of the IQ mixer needs to be performed. The mixer used in the SRON set up is the same as that used in the Cardiff set up (Miteq IRM0118LC1Q). The electrical specifications for this mixer quote a phase balance of \pm 15 degrees and an amplitude balance of $\pm 1.5 \mathrm{~dB}$ [78]. This variation in the conversion losses and phase shift leads to a skewing of the IQ circles that would be seen in a perfect mixer. The mixer is 
therefore calibrated to remove this phase and amplitude error.

The responsivity can also be measured by sweeping each resonator with a VNA at various temperatures. The SRON VNA is calibrated by performing a through calibration operation at a temperature not too far below $T_{c}$. This operation will calibrate the forward transmission of the system and account for losses and phase offsets. Providing the temperature is high enough, the resonators do not couple to the feedline and so do not affect the calibration.

Using data from either the mixer outputs or the VNA allows us to map out the resonance feature in the IQ plane. The IQ circles are translated so that the centre of the lowest temperature resonance lays at the origin. The phase shift is now measured from the centre of the lowest temperature IQ circle and is normalised to the base temperature resonance feature. A schematic of the IQ plane and phase measurement is shown in figure 10.2. Measuring phase in this manner provides a convenient reference point to measure phase from. For a heavily coupled resonator (i.e. I on resonance is $<<1$ ) the phase response is approximated by:

$$
\frac{d \theta}{d N_{q p}} \approx \frac{-2 g Q_{L}}{\omega_{0}}
$$

i.e. twice that calculated in equation 6.4.

Figure 10.3 shows the IQ circles for LEKID 5 as it is warmed from $45 \mathrm{mK}$ to $570 \mathrm{mK}$

The IQ circles were measured as a function of temperature which can be converted to the number of quasi-particles in the meander using equation 3.40. The responsivity is measured by fitting to the linear region of the phase vs quasi- 


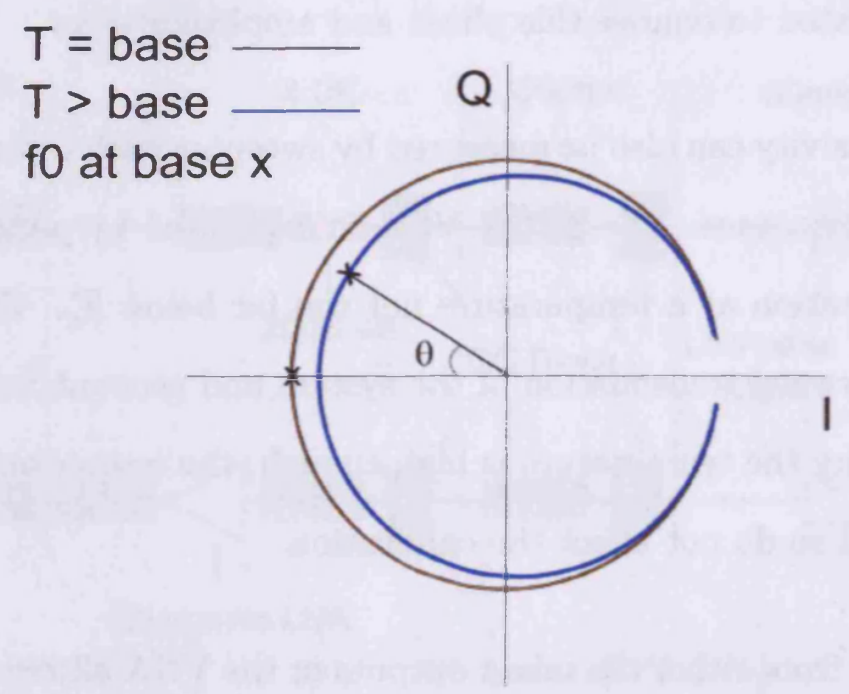

Figure 10.2: The IQ plane for a typical KID device at two temperatures. Both circles are translated so that the centre of the base temperature circle lays on the origin. The phase measured from the origin now has a phase swing of $2 \pi$ as we sweep in frequency about $f_{0}$ at the base temperature.

particle number slope measured from the IQ trajectory as the LEKID was warmed. At low temperatures this film demonstrates back-bending type behaviour. The data points in this region are disregarded in the fitting routine. Figure 10.4 shows the response of LEKID 5. 


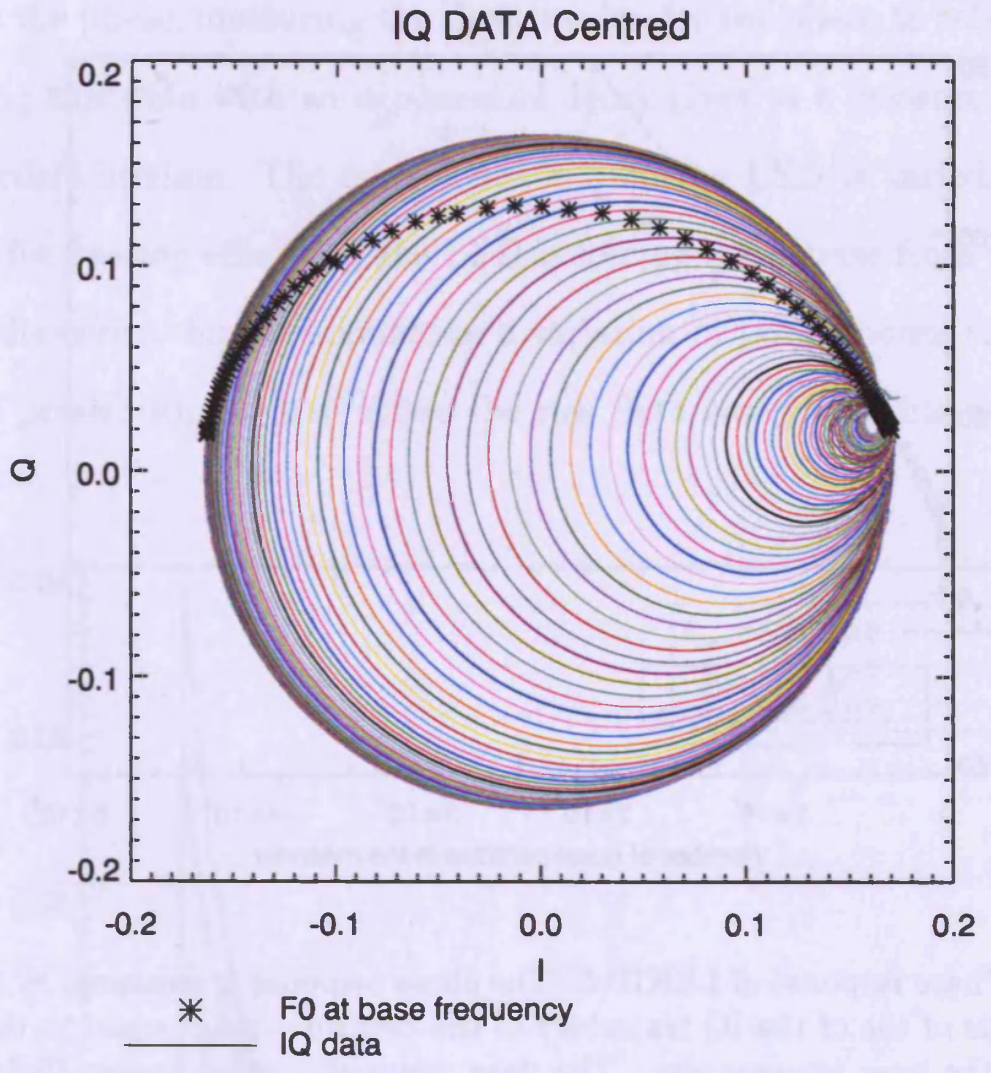

Figure 10.3: The measured IQ circles for LEKID 5 as it is warmed from $45 \mathrm{mK}$ to $570 \mathrm{mK}$ centred on the base temperature IQ circle. The points marked "*"are at the resonant frequency at the base temperature. 


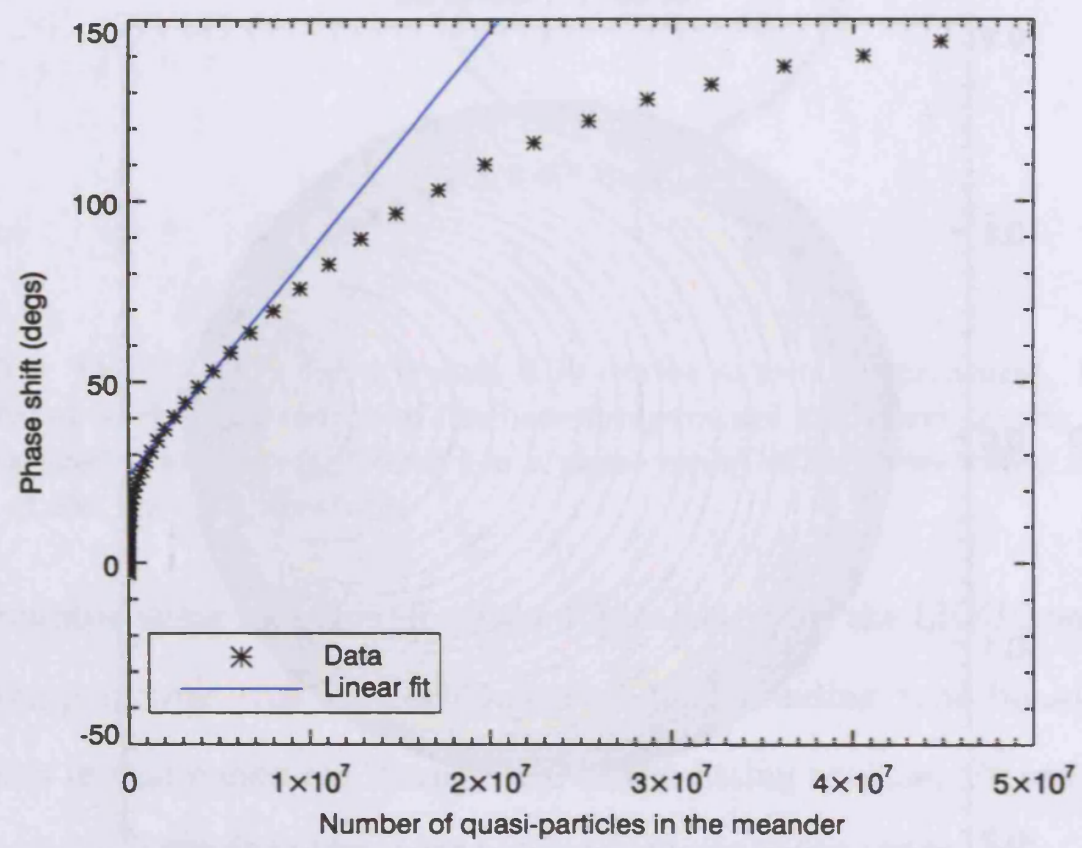

Figure 10.4: Phase response of LEKID 5. The phase response is measured by calculating the phase angle of the of the IQ trajectory of the frequency point equal to the resonant frequency at the base temperature. The data demonstrates an unusual effect at very low temperatures / quasi-particle densities so these points are disregarded. The phase response is found to be linear over a range of about 30 degrees. A linear fit to this region gives a phase response of $6.16 \times 10^{-6}$ degrees per quasi-particle which is equal to $1.075 \times 10^{-7}$ radians per quasi-particle. 


\subsection{Quasi-particle lifetime measurements}

The Quasi-particle lifetime was measured for LEKID 5. This is done by measuring the phase response under the influence of a fast pulse of optical light incident on the chip from an LED. The optical pulse will break a large number of Cooper pairs and shift the phase, measuring the time it takes for the phase to relax back to zero and fitting this data with an exponential decay gives us a measured value for the quasi-particle lifetime. The optical power from the LED is varied. This is done to check for heating effects. If the LED is forcing a response from the LEKID by heating the entire chip we should see a variation in the response time as we vary the LED power. Figure 10.5 shows the raw data along with fits for LEKID 5 at $100 \mathrm{mK}$.

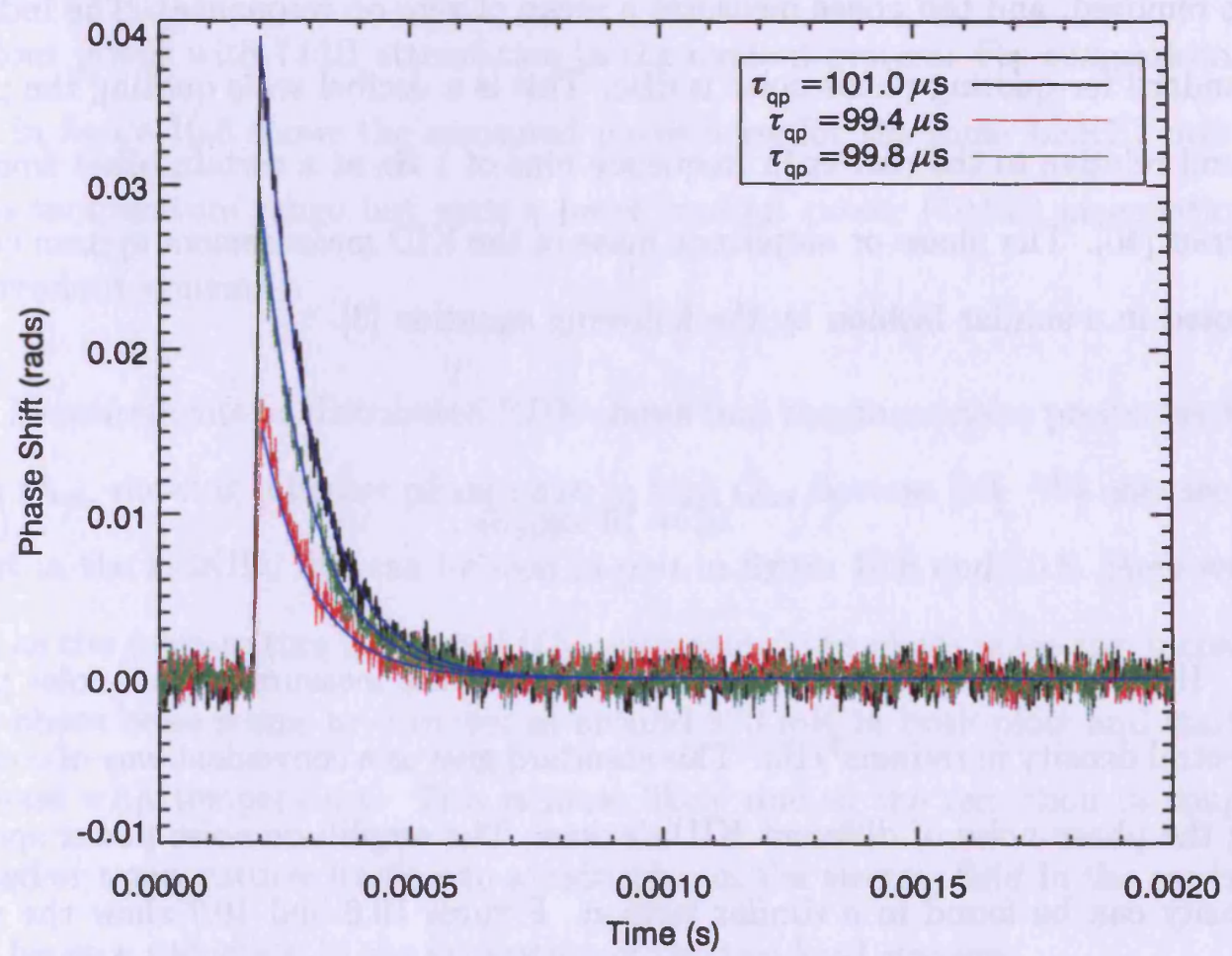

Figure 10.5: The Quasi-particle lifetime measurements for LEKID 5 performed at three different optical pulse power levels. 
Each of the 3 power levels used for the LED measure the same response of around $100 \mu \mathrm{s}$. This proceedure was repeated at each temperature at which the electrical NEP was to be calculated.

\subsection{Phase and amplitude noise measurement}

The phase and amplitude noise of the entire system are measured by taking the Fast Fourier Transform (FFT) of the measured phase and amplitude time streams calculated from the I and Q data. The phase noise is calculated by taking the FFT of $\tan ^{-1}(Q / I)$ of the I and $\mathrm{Q}$ time stream. The amplitude noise is calculated by measuring the change in the radius of the IQ circle (simply $d I$ if all phase offsets are removed, and the phase measures a mean of zero on resonance). The industry standard for quoting phase noise is $\mathrm{dBc}$. This is a decibel scale quoting the power found relative to the carrier in frequency bins of $1 \mathrm{~Hz}$ at a certain offset from the carrier [40]. The phase or amplitude noise of the KID measurement system can be quoted in a similar fashion by the following equation [3].

$$
S_{\theta}^{*}=10 \log _{10} S_{\theta}
$$

Here $S_{\theta}^{*}$ is the phase noise in $\mathrm{dBc}$ and $S_{\theta}$ is the measured phase noise power spectral density in radians ${ }^{2} / \mathrm{Hz}$. This standard give us a convenient way of comparing the phase noise of different KID devices. The amplitude noise power spectral density can be found in a similar fashion. Figures 10.6 and 10.7 show the phase noise and amplitude noise of LEKID 5 respectively measured at different temperatures. 
As the plots in figures 10.6 and 10.7 show, we see a higher level of phase noise when compared to the amplitude noise for the LEKID. This effect is seen in all KID devices and is believed to be associated with a two-level system formed in either the substrate, or an oxide layer on one of the surfaces of the KID $[3,50,77]$. The phase noise also shows a dependance on readout power, showing a reduced effect for higher powers. The scaling of the phase noise with readout power is believed to be due to the saturation of the two-level systems by the microwave electric field at higher readout powers [77]. To measure the lowest NEP for the LEKID device, an optimum readout power must be obtained. This is the highest readout power we can drive the LEKID at before we see the resonant feature being skewed by the device beginning to be driven normal.

The plot in figure 10.6 gives the phase noise power spectral density using a readout power with $74 \mathrm{~dB}$ attenuation in the readout system. For comparison the plot in figure 10.8 shows the measured phase noise for the same LEKID over the same temperature range but with a lower readout power (104dB attenuation in the readout system)

Measurements on distributed KIDs shows that the phase noise properties scale with $Q_{t o t}$, showing a higher phase noise in high $Q_{t o t}$ devices [50]. We also see this effect in the LEKID, this can be seen in plot in figure 10.6 and 10.8. Here we see that as the temperature is reduced ( $Q_{t o t}$ increasing) the phase noise also increases. The phase noise seems to turnover at around $300 \mathrm{mK}$ in both plots and starts to increase with temperature. This is most likely due to the reduction in coupling at higher temperatures leading to a reduction in the electric field in the resonator and hence a reduction in the saturation of the two-level systems. 


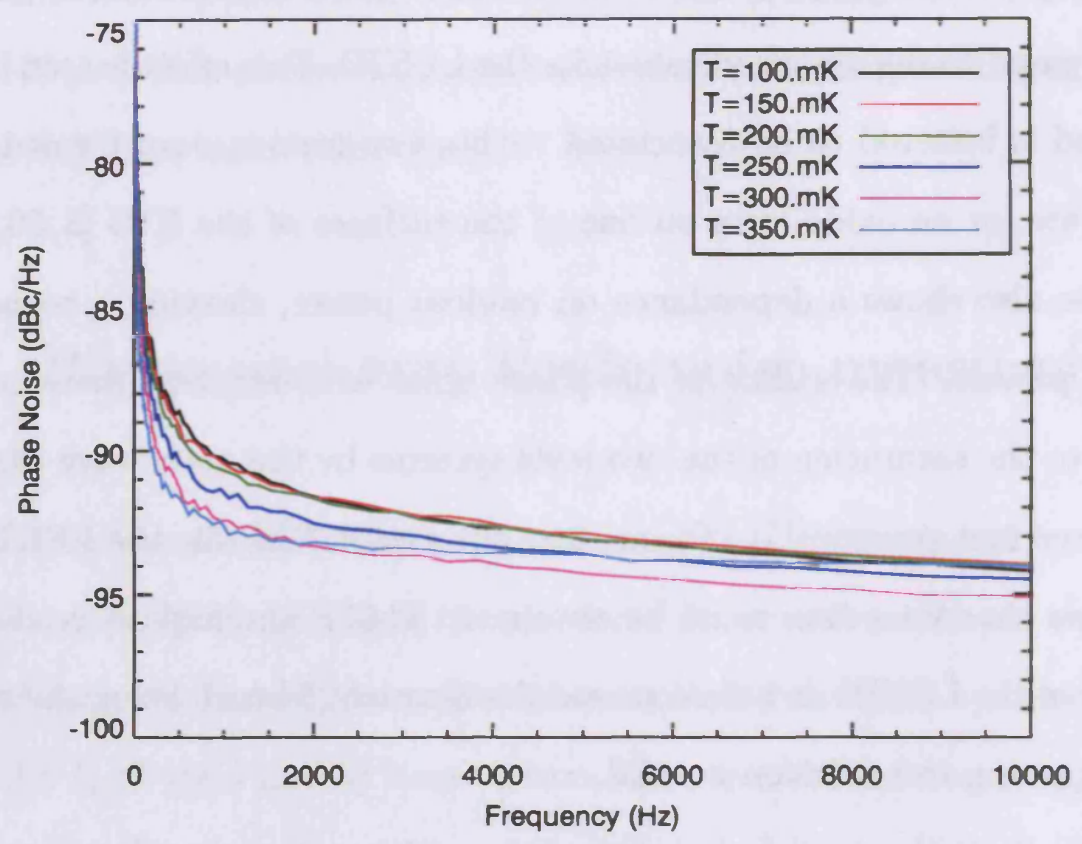

Figure 10.6: Phase noise measurements for LEKID 5.

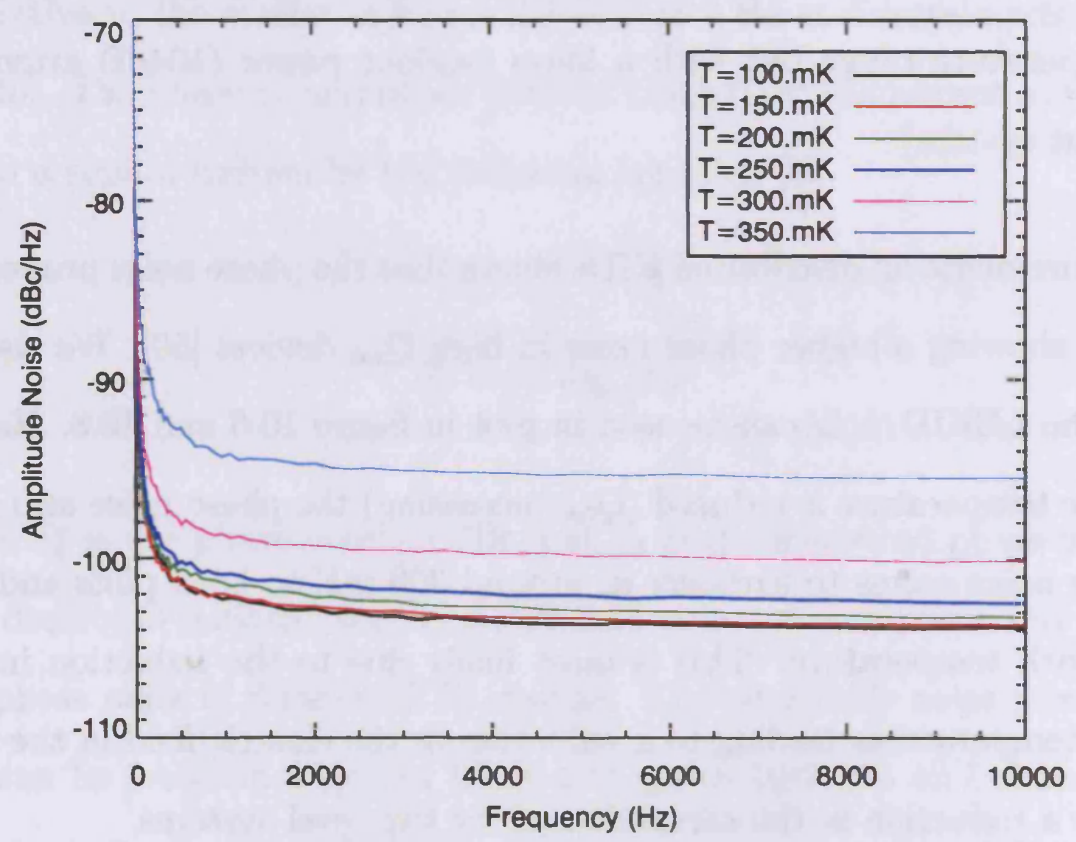

Figure 10.7: Amplitude noise measurements for LEKID 5. 


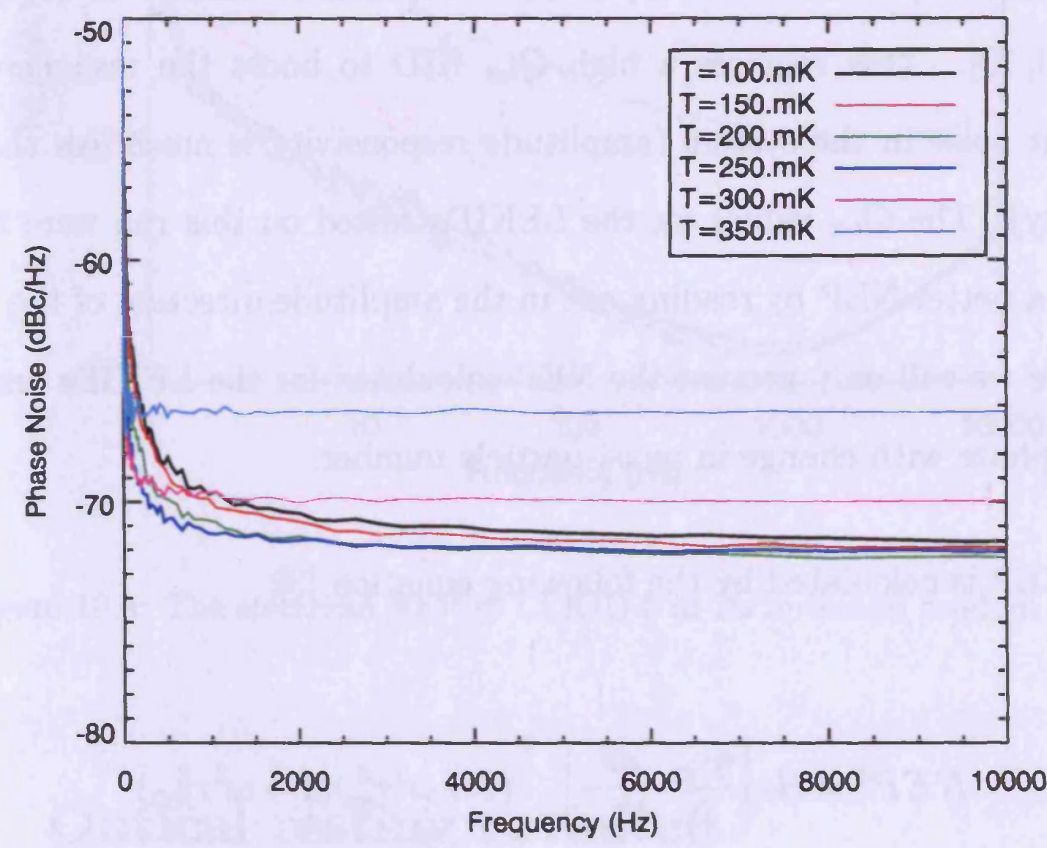

Figure 10.8: Phase noise measurements for LEKID 5 with a low readout power. Here we see an increase in the measured phase noise compared to the phase noise measured in figure 10.6 which was measured at a higher readout power. 


\subsection{NEP Calculation}

Once the responsivity, quasi-particle life-time and phase or amplitude noise are known the NEP can be calculated. The NEP can be measured using the measured responsivity of the amplitude or phase per quasi-particle broken. The excess phase noise measured in KID devices has meant that lower NEPs can be achieved by measuring the response in the amplitude of the KID to change in quasi-particle density $[50,79]$. This requires a high $Q_{\text {tot }}$ KID to boost the responsivity over the readout noise in the system (amplitude responsivity is much less than phase responsivity). The $Q_{t o t}$ values for the LEKIDs tested on this run were too small to achieve a better NEP by reading out in the amplitude direction of the IQ plane and so here we will only present the NEP calculated for the LEKIDs response to change in phase with change in quasi-particle number.

The NEP is calculated by the following equation [3]:

$$
N E P^{2}=S_{\theta}\left[\frac{\eta \tau_{q p}}{\Delta} \frac{d \theta}{d N_{q p}}\right]^{-2}\left(1+\omega^{2} \tau_{q p}^{2}\right)\left(1+\omega^{2} \tau_{R D}^{2}\right)
$$

Here, $S_{\theta}$ is the phase noise power spectral density in $\operatorname{radians}^{2} / \mathrm{Hz}, d \theta / d N_{q p}$ is the phase responsivity in radians per quasi-particle, $\tau_{q p}$ is the quasi-particle lifetime in seconds, $\Delta$ is the gap energy of the superconductor in Joules, $\omega$ is the readout frequency in $\mathrm{Hz}, \tau_{R D}$ is the ring-down time of the resonator in seconds and $\eta$ is an efficiency term set by the Fano factor $[3,80]$. The Fano factor takes into account that the creation of quasi-particles by an absorbed photon is a noisy process due to the fact that quasi-particles and phonons, once created, can interact with each other and so all of the energy from the incident photon is not converted into quasi-particle excitations. $\eta$ is given to be $0.57[3,50,80]$. 
The calculated NEP for this detector at various temperatures in plotted in figures 10.9 and 10.10 .

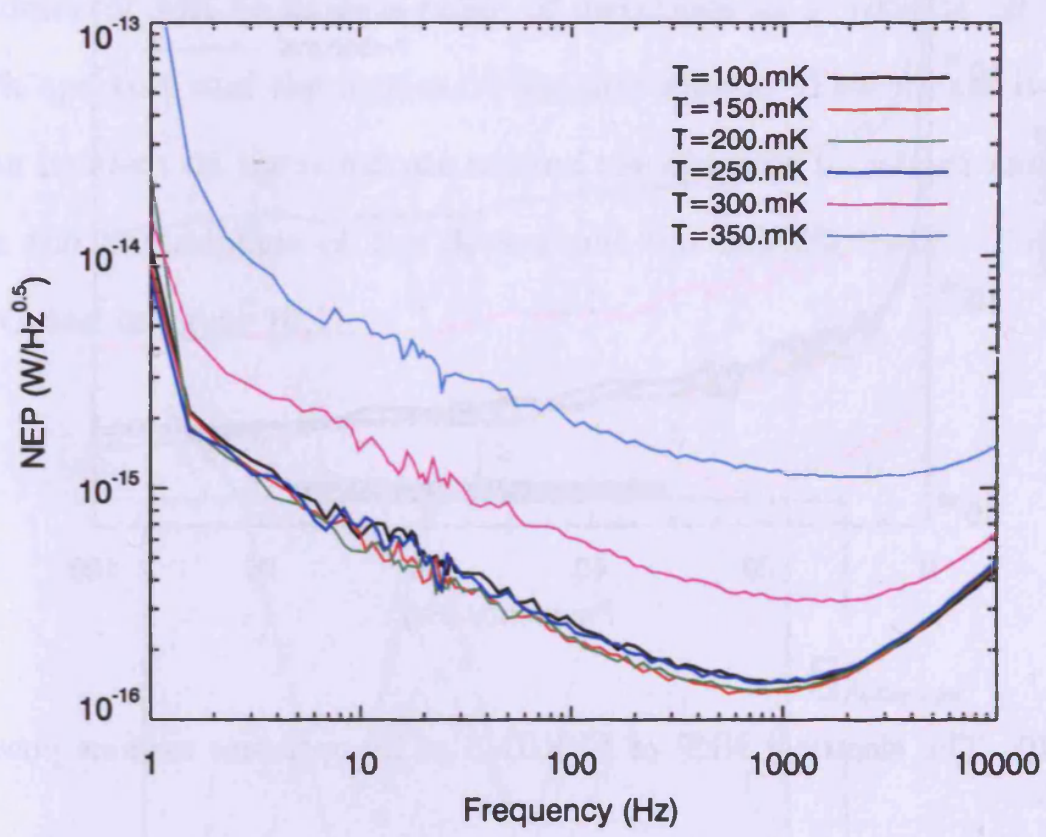

Figure 10.9: The electrical NEP of LEKID 5 at its optimum readout power.

\subsection{Optical testing revisited}

From the measurements of responsivity and quasi-particle lifetime it is possible to calculate the power absorbed during the optical testing performed at Cardiff. During this test we measured the phase from the rotated un-centered IQ data and so the measured phase response is less than that calculated for the SRON response measurements. Measuring the phase response from the data taken at SRON in the same manner as we measured phase for the Cardiff optical testing gives a phase response of $8.8 \times 10^{-8}$ radians per quasi-particle at $280 \mathrm{mK}$. The FFT data taken at Cardiff shows a phase shift of 0.0047 radians when illuminated by the blackbody 


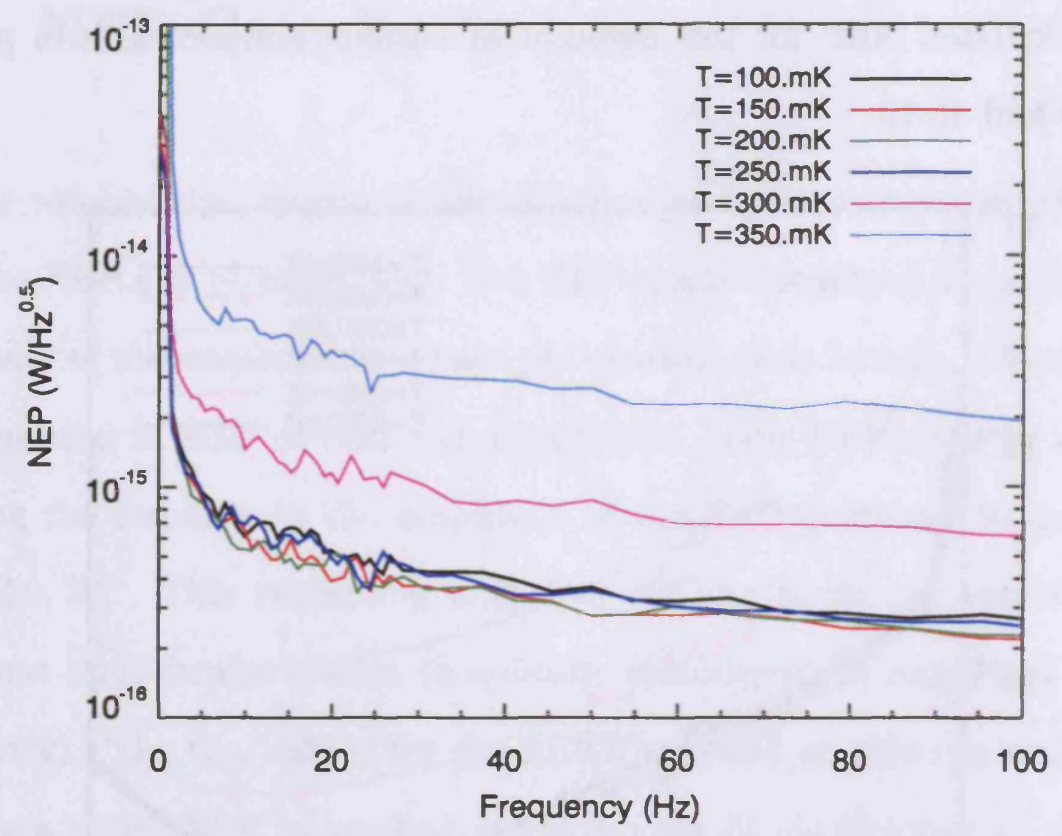

Figure 10.10: The electrical NEP of LEKID 5 at its optimum readout power at low frequencies.

source. The quasi-particle lifetime at $280 \mathrm{mK}$ for this device was $40 \mu \mathrm{s}$. Using this information and equation 3.42 we calculate the excess number of quasi-particles under the optical load from the blackbody to be 53400 . This can be converted into an absorbed power by:

$$
P_{\text {absorbed }}=\frac{N_{x s} \Delta}{\eta \tau_{q p}}=\frac{53400 \times 2.77 \times 10^{23}}{0.57 \times 40^{-6}}=6.48 \times 10^{-14}
$$

This calculated absorbed power is greater than predicted. Using the estimated blackbody power incident on the LEKID from table 9.3, the predicted optical efficiency from the plot in figure 9.16 and the fact that the LEKID should only be sensitive to radiation polarized parallel to the meander, gives us an upper limit on the estimated absorbed power of the order of $2.3 \times 10^{-14} \mathrm{~W}$. The discrepancy in these results is believed to lie in some of the crude assumptions made in the 
optical model, this mainly being that the incident radiation is of the form of a collimated beam perpendicular to the substrate. In reality the radiation incident on the detector will be from a range of directions as it reflects off the walls of the thick aperture and the insides of the chip mount. This makes it possible for radiation incident on the substrate around the detector to refract and be trapped between the groundplane of the device and the LEKID itself. This process is demonstrated in figure 10.11 .

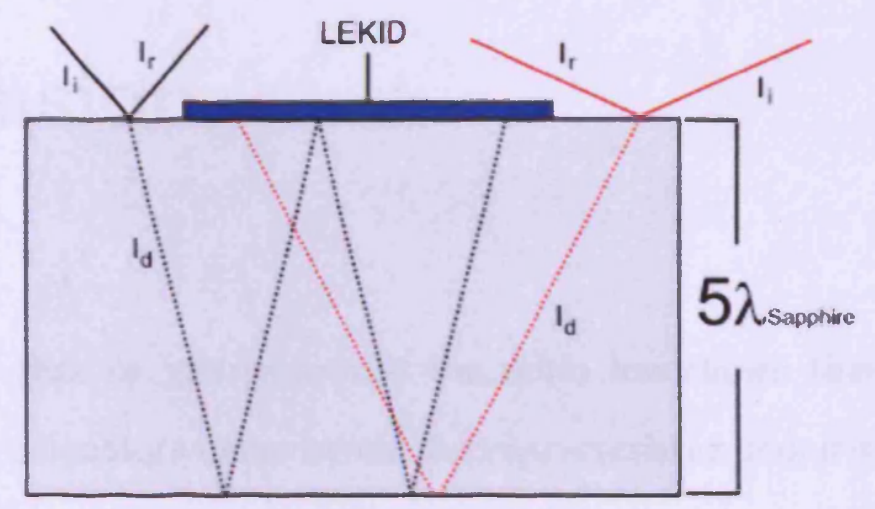

Figure 10.11: Schematic of stray light absorption in LEKID 5. Here radiation incident on the substrate around the LEKID $\left(I_{i}\right)$ can be refracted and trapped between the LEKID and the groundplane $\left(I_{d}\right)$. The light that is reflected off the substrate may reflect around the chip mount and be absorbed by the LEKID directly or through this trapping process.

The substrate for this device was $325 \mu \mathrm{m}$ sapphire. The high refractive index of the substrate along with the thickness being of order $5 \lambda$ makes it possible for light to be absorbed from a wide range of angles. The light trapped under the LEKID can now be incident on the LEKID several times greatly increasing its chances of absorption. This scenario also creates a greater effective absorbing area for the LEKID leading to more power being absorbed. This effect is very difficult to quantify given that we only have an estimate of the blackbody temperature and no way of predicting the reflections within the chip mount, substrate and aperture. However, the explanation seems the most likely as $40 \mathrm{~K}$ is a realistic upper limit 
for the blackbody temperature and electrical pickup has been ruled out. 


\section{Chapter 11}

\section{Conclusion}

Work done to date by groups around the world has shown that microwave superconducting resonators demonstrate the characteristics required to be a strong candidate for the next generation of ultra-sensitive detectors for use in large multiplexed arrays. Recent results demonstrate typical MKID devices with electrical NEPs of $2.5 \times 10^{-18} \mathrm{~W} / \sqrt{\mathrm{Hz}}$ using an amplitude readout scheme [50]. These levels of sensitivity are rapidly approaching the the requirements for future satellite missions observing in the MIR-FIR bands and already exceed requirements for a photon noise limited ground based system operating in the available atmospheric windows of $200 \mu \mathrm{m}, 350 \mu \mathrm{m}$ and $450 \mu \mathrm{ms}$. While these levels of sensitivity are impressive, a suitable optical coupling scheme in these wavebands is yet to be realised.

Simulations show that the LEKID device can be designed to allow good optical coupling to FIR-mm-wave radiation as a distributed absorber with a matched free space impedance. A model to simulate absorption based upon a quasi-optical approach has been developed. This model allows us to define the properties required 
of the LEKID for good optical efficiency and shows that good optical coupling can be obtained using practical substrates and anti-reflection coatings in conjunction with a hybrid LEKID made from two different superconducting metals.

The electrical NEP measurements made at SRON show that the prototype LEKID device fabricated at Cardiff had a minimum NEP of around $1.5 \times 10^{-16} \mathrm{~W} / \sqrt{\mathrm{Hz}}$ at $1000 \mathrm{~Hz}$ using a phase readout with a large $1 / \mathrm{f}$ noise component limiting the NEP to around $5 \times 10^{-15}-7 \times 10^{-16} \mathrm{~W} / \sqrt{\mathrm{Hz}}$ for frequencies between 1 and $10 \mathrm{~Hz}$. This is a long way from the standard set by the MKID to date but is very encouraging. We know that the film used for this test was of poor quality and hence had low a $\mathrm{Q}$ factor and quasi-particle lifetime. This prohibited a gain in sensitivity using an amplitude readout. Aluminium films fabricated at SRON have shown quasiparticle lifetimes in excess of $400 \mu \mathrm{s}$, a factor of 4 better than the film used in this measurement [51]. Using one of these high quality films alone would gain the LEKID around a factor of 4 in sensitivity for a phase readout.

The microwave theory of the LEKID discussed throughout this thesis suggests that there is no reason why the LEKID should not perform as well as the MKID fabricated from the same materials and operating at the same temperature. From this point of view we are confident that the sensitivity of the LEKID can be improved upon considerably and that there is no fundamental reason why the LEKID cannot meet the requirements for future satellite FIR missions.

The LEKID is still in an early stage of development and has many properties which are yet to be optimised. These include:

- The detector impedance. The microwave impedance of the detector on resonance is given by the ratio of magnetic to electric fields $(Z=\sqrt{L / C})$. This is a property that is easily varied in the LEKID by altering the spacing in 
the meander or inter-digital capacitor. Altering the impedance could be used to reduce the phase noise seen in KID devices. The level of the phase noise in both the MKID and the LEKID is suppressed with an increase in readout power [77]; this is believed to be due to the saturation of two-level systems in the dielectric layers surrounding a KID. The increase in readout power is limited by the critical current in the KID being exceeded and driving the KID normal. By increasing the impedance of the resonator it may be possible to produce the high fields required to saturate two-level systems while still remaining below the critical current of the resonator.

- Different microwave coupling regimes. The devices simulated and tested in this thesis were coupled to microstrip feedlines and showed that the resonator had to be at a distance of around $400 \mu \mathrm{m}$ to achieve loaded quality factors of order 100000 or more. This distance leaves a lot of dead space between the resonator and the feedline which would severely reduce the filling factor of an array. Coupling to a CPW feedline could be a nice solution to this problem. The stray fields in a CPW line extend over a far shorter distance than to that of a microstrip line for a give substrate thickness. This means that the LEKID needs to be pulled in far closer to the feedline to achieve suitable microwave coupling, hence reducing the dead area in a focal plane array. CPW lines are also far easier to fabricate requiring only a single deposition on the substrate surface leaving the backside of the substrate clear for back illumination. CPW lines also provide convenient ways of producing a narrow to wide line transition without changing the impedance of the line. This characteristic is very useful for producing wide wire-bond pads for connecting to the chip. CPW coupled devices have recently been simulated in Sonnet and indicate that they should work well. Testing of these devices will be underway soon. 
- Substrate materials. So far we have only produced LEKIDs on solid sapphire substrates. There is no fundamental reason for this and it may prove beneficial to change the substrate material. Silicon for example can be etched using a wet or dry method. Etching of the silicon substrate could be used to remove areas of substrate contributing to noise, or, with an additional silicon-nitride layer, silicon could be used to produce LEKID devices on thermally isolated webs. This would increase the quasi-particle lifetime through phonon re-trapping and hence increase the sensitivity of our detector. Other substrates should also be investigated, substrates like Diamond-like-Carbon can be sputtered into thin layers and could well reduce the phase noise properties of any KID device.

- Superconducting materials. The choice of superconducting materials was considered when discussing optical coupling to the LEKID. Development of the LEKID will require a study of a wide range of superconductors, substrates, and deposition techniques as each will vary in its normal state conductivity and quasi-particle lifetime.

The LEKID design is particularly well suited for use in large format arrays not only from a multiplexing point of view but also a fabrication and packing point of view. The multiplexing readout electronics for the MKID have already been demonstrated for the Caltech MKID demonstration camera on the CSO [4], and now, the entire MKID community has put a clear emphasis on the development for a readout system capable of reading out kilo-pixel arrays. Such a technology would be directly transferable to a LEKID array. The LEKID array is as simple as possible to fabricate, requiring just a single deposition and etch for a single material device fed by a CPW line and two deposition and etches for a hybrid LEKID with similar microwave coupling. The simplicity in fabrication will help 
insure a high yield of detectors with low cost and a quick turnaround. Unlike the MKID the majority of the LEKID serves as the active pixel area. This allows the LEKID to be packed extremely closely forming a simple array with high filling factor. A concept array is shown in figure 11.1.

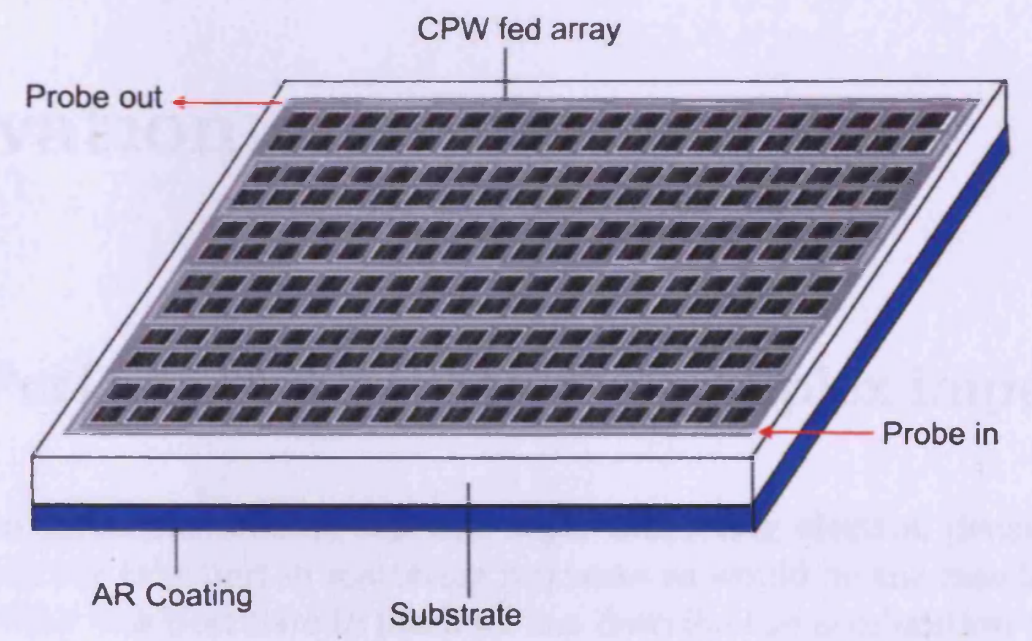

Figure 11.1: CPW fed LEKID array concept

Throughout this thesis the emphasis has very much been upon the use of the LEKID as a FIR astronomical detector. However suitable scaling of the meander could make the LEKID just as viable for longer wavelengths extending into the mm. MKIDs have also been considered for dark matter experiments, where Weakly Interacting Massive Particles (WIMPs) generate phonons in the substrate of the MKID chip which in turn break Cooper pairs in the resonator. The lack of ability to direct phonons to the sensitive tip in an MKID using this detection method means that the LEKID (which has no position dependance) may be better suited. The LEKID also has potential applications in industrial THz imaging and Spectroscopy. 


\section{Appendix A}

\section{Derivation of equations}

\section{A.1 Perfect conductivity and complex impedance}

The London equations assume that the superconducting electron density $\left(n_{s}\right)$ is one that does not take part in scattering processes as would be the case for normal electrons. With this postulate in place we can describe the acceleration of a single electron in a field $\mathbf{E}$ by:

$$
m \frac{d v}{d t}=-e \mathbf{E} \rightarrow \frac{d v}{d t}=\frac{-e \mathbf{E}}{m}
$$

here $m$ and $e$ are the mass and charge of an electron respectively.

Current density $\mathbf{J}$ is simply described by the amount of charge passing through the cross sectional area of the current carrying medium per second. This can be written in terms of the electron velocity $(v)$ and number density $(n)$. Taking the time derivative of $\mathbf{J}$ and substituting in to A.1 gives :

$$
\mathbf{J}=-n e v \rightarrow \frac{d \mathbf{J}}{d t}-n e \frac{d v}{d t}
$$

This leads to the first London equation which describes perfect conductivity.

$$
\frac{d \mathbf{J}}{d t}=\frac{n e^{2}}{m} \mathbf{E}
$$


We can write $\mathbf{J}$ as a time varying current as it would be in an AC circuit

$$
\mathbf{J}=\mathbf{J}_{\mathbf{0}} e^{j \omega t} \rightarrow \frac{d \mathbf{J}}{d t}=j \omega \mathbf{J}_{\mathbf{0}} e^{j \omega t} \rightarrow \frac{d \mathbf{J}}{d t}=j \omega \mathbf{J}
$$

we can now write A.3 in terms of a conductivity $\sigma$ and a stimulus $\mathbf{E}$

$$
\mathrm{J}=-j \frac{n e^{2}}{\omega m} \mathbf{E} \rightarrow \sigma=-\mathrm{j} \frac{\mathrm{e}^{2}}{\omega \mathrm{m}}
$$

\section{A.2 The Meissner effect}

As mentioned in chapter 3 the London model is not really a derivation but a set of conditions that describe the effects seen in superconductivity. Here we will follow the derivation given by Rose-Innes and Rhoderick [30].The Meissner effect can be describe in the similar way by adding a set of conditions to Maxwell's equations to satisfy the observed effect. It should be noted that in this derivation we are describing the Meissner effect by finding a set of solutions to Maxwell's equations for a magnetic field applied to a semi-infinite superconducting medium like that shown in figure A.1. These solutions are not valid for the case of a thin film which is dealt with in the next section. We start by stating Maxwell's equations for Faraday's law of induction and Ampéres circuital law.

$$
\begin{array}{r}
\nabla \times \mathbf{E}=-\mu_{0} \frac{\partial \mathbf{H}}{\partial \mathrm{t}}=\mathrm{curl} \mathbf{E} \\
\nabla \times \mathbf{H}=\mathbf{J}=\mathrm{curl} \mathbf{H}
\end{array}
$$

Applying A.5 to a perfect conductor (equation A.3) gives us

$$
\dot{\mathbf{H}}=\frac{-\mathrm{m}}{\mu_{0} \mathrm{ne}^{2}} \operatorname{curl} \dot{\mathbf{J}}
$$

Using Ampéres law we can write A.7 in terms of $\dot{\mathbf{H}}$ alone: 


$$
\dot{\mathbf{J}}=\operatorname{curl} \dot{\mathbf{H}} \rightarrow \dot{\mathbf{H}}=\frac{-\mathrm{m}}{\mu_{0} \mathrm{ne}^{2}} \operatorname{curl}(\operatorname{curl} \dot{\mathbf{H}})
$$

Calling $m / \mu_{0} n e^{2}=\alpha$ for simplicity we have

$$
\begin{gathered}
\dot{\mathbf{H}}=-\alpha \operatorname{curl}(\operatorname{curl} \dot{\mathbf{H}}) \\
\operatorname{curl}(\operatorname{curl} \dot{\mathbf{H}})=\operatorname{grad}(\operatorname{div}(\dot{\mathbf{H}}))-\nabla^{2} \dot{\mathbf{H}}
\end{gathered}
$$

From Maxwell's equation for Gauss's law $\operatorname{div}(\dot{\mathbf{H}})=\mathbf{0}$ so we are left with:

$$
\begin{array}{r}
\dot{\mathbf{H}}=\alpha \nabla^{2} \dot{\mathbf{H}} \\
\dot{\mathbf{H}} \frac{\mathbf{1}}{\alpha}=\nabla^{2} \dot{\mathbf{H}}=\frac{\partial^{2} \dot{\mathbf{H}}}{\partial \mathbf{x}^{2}}
\end{array}
$$

This has solutions

$$
\dot{\mathbf{H}}(\mathbf{x})=\dot{\mathbf{H}}(\mathbf{a}) \exp \left(\frac{ \pm \mathbf{x}}{\sqrt{\alpha}}\right)
$$

Of the two solution only one makes physical sense $(\exp (-x / \sqrt{\alpha})$. The other solution goes to infinity as $x$ increases. The valid solution shows that a time variying magnetic field with value $\mathbf{H}_{\mathbf{a}}$ at the surface will decays as $(\exp (-x / \sqrt{\alpha})$ as we move from the surface of the perfect conductor. This solution is not however the observed effect we see in a superconductor. The Meissner effect shows a rapid decay of all magnetic fields (static and time varying) as we move from the surface of a superconductor. This implies that we need to apply a set of conditions to A.5 to account for this. In fact if we choose to integrate A.7 under the certain conditions that we set the constants of integration to zero so that:

$$
\mathbf{H}=\frac{-\mathrm{m}}{\mu_{0} \mathrm{ne}^{2}} \operatorname{curl} \mathbf{J} .
$$


Equation A.11 now takes the form:

$$
\begin{array}{r}
\mathbf{H}=\alpha \nabla^{2} \mathbf{H} \\
\mathbf{H} \frac{\mathbf{1}}{\alpha}=\nabla^{2} \mathbf{H}=\frac{\partial^{2} \mathbf{H}}{\partial \mathbf{x}^{2}} .
\end{array}
$$

which has the solution:

$$
\mathbf{H}(\mathbf{x})=\mathbf{H}(\mathbf{a}) \exp \left(\frac{-\mathbf{x}}{\sqrt{\alpha}}\right)
$$

This new expression for $\mathbf{H}(\mathbf{x})$ now demonstrates a rapid decay of all fields as we move from the surface of a superconducting material. Substituting back in for $\alpha$ gives

$$
\mathbf{H}(\mathbf{x})=\mathbf{H}(\mathbf{a}) \exp \left(\frac{-\mathbf{x}}{\sqrt{\mathrm{m} / \mu_{0} \mathrm{ne}^{2}}}\right)
$$

The external field falls to $1 / e$ of its value at the surface in the distance $\sqrt{m / \mu_{0} n e^{2}}$, which is known as the London penetration depth $\left(\lambda_{L}\right)$.

$$
\lambda_{L}=\sqrt{\frac{m}{\mu_{0} n e^{2}}}
$$

\section{A.3 Kinetic and internal magnetic inductance}

In the case where the film thickness is not in the limit $t<<\lambda$ or $t>>\lambda$ (as will be the case for most practical films used in the LEKID) we need to solve for the current density within the film volume. Here we will follow the derivation of Porch [81]. This derivation looks at the magnetic fields generated by a current flowing through a superconducting strip modified by the condition that the field decays rapidly from the surface of the film as shown in the last section. Figure A.2 shows the cross section of a strip of superconductor. We define the $\mathrm{x}$ axis through the strip centre with the top and bottom surfaces being $t / 2$ from the centre. The 


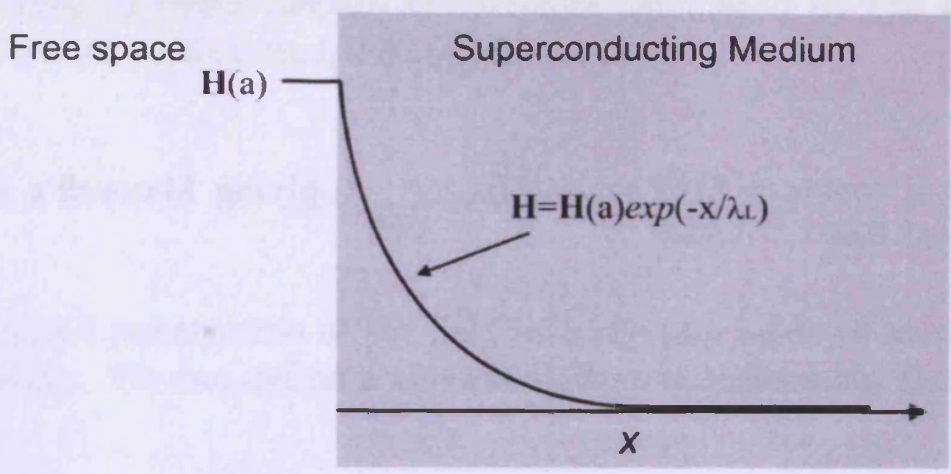

Figure A.1: Schematic of the decay of an applied field to a superconductor surface current is into the page giving the fields $\mathbf{H}$ just outside the film volume.

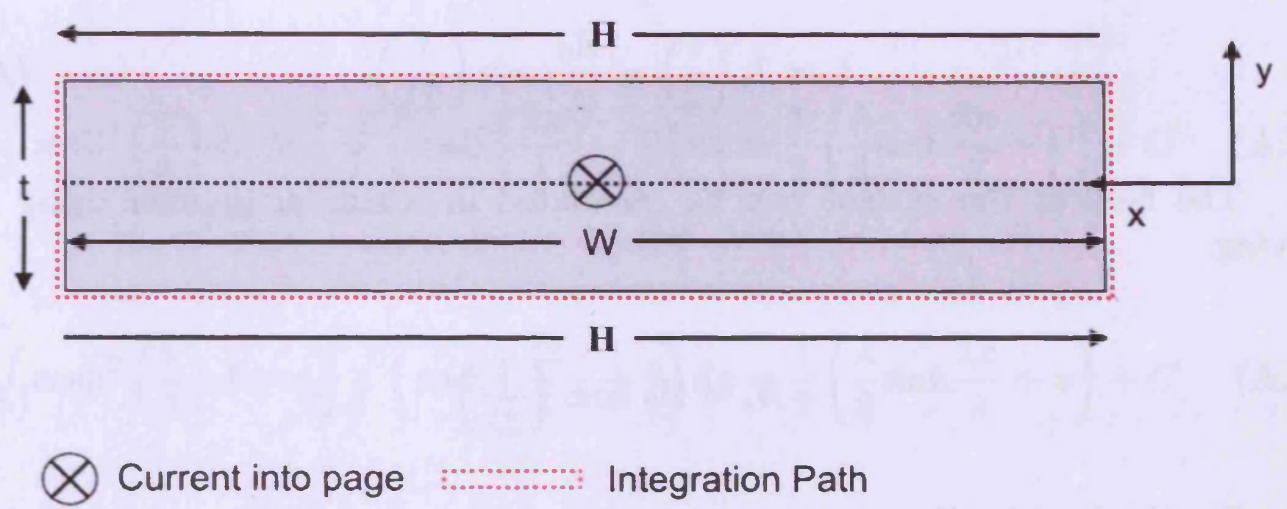

Figure A.2: Superconducting strip cross-section

Using the equations A.14 and A.17 we can describe the fields inside a semiinfnite superconductor as:

$$
\nabla^{2} \mathbf{H}=\frac{1}{\lambda^{2}} \mathbf{H}
$$

The strip in A.2 has a different solution to the field distribution to that of an infinite sample. The fields above and below the film are in opposite directions, and, just as in the case for a normal film or wire the field is zero in the centre. The solution under these boundary conditions is: 


$$
H_{y}(\mathrm{x})=\mathrm{H}_{0} \sinh \left(\frac{\mathrm{x}}{\lambda}\right)
$$

Here $H_{0}$ is a constant field scaling factor. Applying Maxwell's equation for Ampéres circuital law

$$
\nabla \times \mathbf{H}=\mathbf{J}
$$

gives

$$
J_{z}=\frac{\partial \mathrm{H}_{\mathbf{y}}}{\partial \mathbf{x}}=\frac{\mathrm{H}_{0}}{\lambda} \cosh \left(\frac{\mathbf{x}}{\lambda}\right)
$$

We can use A.21 to calculate the current at the surface which is at $x=t / 2$.

$$
J_{s}=\mathrm{J}_{\mathrm{z}}\left(\frac{\mathrm{t}}{2}\right)=\frac{\mathrm{H}_{0}}{\lambda} \cosh \left(\frac{\mathrm{t}}{2 \lambda}\right)
$$

The field at the surface can be calculated in a similar manner using A.19 giving:

$$
H_{s}=\mathrm{H}_{0} \sinh \left(\frac{\mathrm{t}}{2 \lambda}\right)
$$

Solving A.22 for $\mathbf{H}_{0}$ and substituting into A.23 and noting that $\sinh (x) / \cosh (x)=\tanh (x)$ gives:

$$
H_{s}=\mathrm{J}_{\mathrm{s}} \lambda \tanh \left(\frac{\mathrm{t}}{2 \lambda}\right)
$$

We can calculate the current in the strip by applying Ampéres circuital law to the line integral shown in figure A.2. Here we take the surface value of the field and multiply it by the strip width $(W)$.

$$
I=2 W H_{0} \sinh \left(\frac{t}{2 \lambda}\right)
$$


The average current density is therefore calculated by the total current $(I)$ divided by the cross-sectional area $(W t)$

$$
\bar{J}=\frac{I}{W t}=\frac{2 H_{0}}{t} \sinh \left(\frac{t}{2 \lambda}\right)
$$

The limited penetration of the field into the film leads to an enhanced surface current density. We can define a surface current enhancement factor $(f)$ as:

$$
f=\frac{J_{s}}{\bar{J}}=\frac{t}{\lambda} \operatorname{coth}\left(\frac{t}{2 \lambda}\right)
$$

Here we have made use of $\cosh (x) / \sinh (x)=\operatorname{coth}(x)$.

To simplify expressions later in in this derivation it is worth defining two sets of standard integral equivalents.

$$
\begin{aligned}
& \int \sinh ^{2}\left(\frac{x}{\lambda}\right) d x=\frac{1}{2} \int\left(\cosh \left(\frac{2 x}{\lambda}\right)-1\right) d x=\frac{1}{2}\left(\frac{\lambda}{2} \sinh \frac{2 x}{\lambda}-x\right)+C \\
& \int \cosh ^{2}\left(\frac{x}{\lambda}\right) d x=\frac{1}{2} \int\left(\cosh \left(\frac{2 x}{\lambda}\right)+1\right) d x=\frac{1}{2}\left(\frac{\lambda}{2} \sinh \frac{2 x}{\lambda}+x\right)+C
\end{aligned}
$$

We can now calculate the Kinetic inductance using the standard formula mentioned in chapter 3 (equation 3.16). However we now have an expression for the current density across the cross-sectional area and no longer need to make the assumptions made for $t<\langle\lambda$ and $t>>\lambda$ as we did in equations 3.17 and 3.18. Taking the current distribution described in A.21 we can write:

$$
L_{k} I^{2}=\int_{s} \mu_{0} \lambda^{2} J_{z}^{2} \mathrm{ds}=\mu_{0} \lambda^{2} \mathrm{~W} \int_{-\mathrm{t} / 2}^{\mathrm{t} / 2} \frac{\mathrm{H}_{0}^{2}}{\lambda^{2}} \cosh ^{2}\left(\frac{\mathrm{x}}{\lambda}\right) \mathrm{dx}
$$

From A.25 we have and expression for $I$ giving: 


$$
4 W^{2}{H_{0}}^{2} \sinh ^{2}\left(\frac{t}{2 \lambda}\right) L_{k}=\mu_{0} W{H_{0}}^{2}\left(\frac{t}{2}\right)\left[\left(\frac{\lambda}{t}\right) \sinh \left(\frac{t}{\lambda}\right)+1\right]
$$

Solving for $L_{k}$ gives

$$
L_{k}=\frac{\mu_{0} t}{8 W} \frac{(\lambda / t) \sinh (t / \lambda)+1}{\sinh ^{2}(t / 2 \lambda)}=\frac{\mu_{0} \lambda}{4 W} \frac{\cosh (t / 2 \lambda) \sinh (t / 2 \lambda)+t / 2 \lambda}{\sinh ^{2}(t / 2 \lambda)}
$$

Using $\cosh (x) / \sinh (x)=\operatorname{coth}(x)$ and $1 / \sinh (x)=\operatorname{cosec}(x)$ we can rewrite A.32 as:

$$
L_{k}=\frac{\mu_{0} \lambda}{4 W}\left[\left(\operatorname{coth}\left(\frac{t}{2 \lambda}\right)\right)+\left\{\left(\frac{t}{2 \lambda}\right) \operatorname{cosec}^{2}\left(\frac{t}{2 \lambda}\right)\right\}\right]
$$

The magnetic inductance can be found in a similar way as the kinetic inductance. By definition we can write magnetic inductance $\left(L_{m}\right)$ as:

$$
L_{m} I^{2}=\mu_{0} \int_{s} H_{y}^{2} \mathrm{ds}=\mu_{0} \mathrm{H}_{0}^{2} \mathrm{~W} \int_{-\mathrm{t} / 2}^{\mathrm{t} / 2} \sinh ^{2}\left(\frac{\mathrm{x}}{\lambda}\right) \mathrm{dx}
$$

Here we need to integrate the field over the cross-sectional area. This can be done using A.19 and adding the expression for current $I$ from A.25. The sinh term leads us to use the integrals of A.28 giving:

$$
4 W^{2} H(0)^{2} \sinh ^{2}\left(\frac{t}{2 \lambda}\right) L_{m}=\mu_{0} W H(0)^{2}\left(\frac{t}{2}\right)\left[\left(\frac{\lambda}{t}\right) \sinh \left(\frac{t}{\lambda}\right)-1\right]
$$

This takes the same form as A.31 apart from a -1 term instead of $a+1$ term. This leads us to $L_{m}$ taking a similar form as A.33 and is written as:

$$
L_{m}=\frac{\mu_{0} \lambda}{4 W}\left[\left(\operatorname{coth}\left(\frac{t}{2 \lambda}\right)\right)-\left\{\left(\frac{t}{2 \lambda}\right) \operatorname{cosec}^{2}\left(\frac{t}{2 \lambda}\right)\right\}\right]
$$




\section{A.4 Transmission line equations}

The ABCD matrix for a transmission line of length $l$ is given by

$$
\begin{array}{cl}
A=\cos (\beta l) & B=j Z_{0} \sin (\beta l) \\
C=j Y_{0} \sin (\beta l) & D=\cos (\beta l)
\end{array}
$$

The ABCD matrix for a shunt impedance to ground is given by

$$
\begin{array}{cc}
A=1 & B=0 \\
C=Y_{0} & D=1
\end{array}
$$

Where

$$
\begin{aligned}
Z_{0} & =\frac{377}{\sqrt{\epsilon_{r}}} \\
Y_{0} & =\frac{1}{Z_{0}} \\
\nu_{p} & =\frac{c}{\sqrt{\epsilon_{r}}} \\
\beta & =\frac{\omega}{\nu_{p}} \\
\lambda & =\frac{2 \pi}{\beta}
\end{aligned}
$$




\section{Bibliography}

[1] K. D. Irwin and G. C. Hilton. Cryogenic Particle Detection. Unknown, 2005.

[2] P. K. Day, H. G. LeDuc, B. A. Mazin, A. Vayonakis, and J. Zmuidzinas. A broadband superconducting detector suitable for use in large arrays. Nature, 425:817-821, October 2003.

[3] B. A. Mazin. Microwave kinetic inductance detectors. PhD thesis, California Institute of Technology, United States - California, 2005.

[4] J. Schlaerth, A. Vayonakis, P. Day, J. Glenn, J. Gao, S. Golwala, S. Kumar, H. Leduc, B. Mazin, J. Vaillancourt, and J. Zmuidzinas. A Millimeter and Submillimeter Kinetic Inductance Detector Camera. Journal of Low Temperature Physics, pages 113-+, January 2008.

[5] A. V. Sergeev, V. V. Mitin, and B. S. Karasik. Ultrasensitive hot-electron kinetic-inductance detectors operating well below the superconducting transition. Applied Physics Letters, 80(5):817-819, 2002.

[6] B. A. Mazin, B. Bumble, P. K. Day, M. E. Eckart, S. Golwala, J. Zmuidzinas, and F. A. Harrison. Position sensitive x-ray spectrophotometer using microwave kinetic inductance detectors. Applied Physics Letters, 89:2507-+, November 2006.

[7] C. D. Dowell, C. A. Allen, R. S. Babu, M. M. Freund, M. Gardner, J. Groseth, M. D. Jhabvala, A. Kovacs, D. C. Lis, S. H. Moseley, Jr., T. G. Phillips, R. F. Silverberg, G. M. Voellmer, and H. Yoshida. SHARC II: a Caltech submillimeter observatory facility camera with 384 pixels. In T. G. Phillips and J. Zmuidzinas, editors, Millimeter and Submillimeter Detectors for Astronomy. Edited by Phillips, Thomas G.; Zmuidzinas, Jonas. Proceedings of the SPIE, Volume 4855, pp. 73-87 (2003)., volume 4855 of Presented at the Society of Photo-Optical Instrumentation Engineers (SPIE) Conference, pages 73-87, February 2003.

[8] D. Ward-Thompson, P. A. R. Ade, H. Araujo, I. Coulson, J. Cox, G. R. Davis, R. Evans, M. J. Griffin, W. K. Gear, P. Hargrave, P. Hargreaves, D. Hayton, 
B. J. Kiernan, S. J. Leeks, P. Mauskopf, D. Naylor, N. Potter, S. A. Rinehart, R. Sudiwala, C. R. Tucker, R. J. Walker, and S. L. Watkin. First groundbased $200-\mu \mathrm{m}$ observing with THUMPER on JCMT - sky characterization and planet maps. MNRAS, 364:843-848, December 2005.

[9] G. L. Pilbratt. Herschel Space Observatory mission overview. In J. C. Mather, editor, IR Space Telescopes and Instruments. Edited by John C. Mather. Proceedings of the SPIE, Volume 4850, pp. 586-597 (2003)., volume 4850 of Presented at the Society of Photo-Optical Instrumentation Engineers (SPIE) Conference, pages 586-597, March 2003.

[10] G. Bagnasco, M. Kolm, P. Ferruit, K. Honnen, J. Koehler, R. Lemke, M. Maschmann, M. Melf, G. Noyer, P. Rumler, J.-C. Salvignol, P. Strada, and M. Te Plate. Overview of the near-infrared spectrograph (NIRSpec) instrument on-board the James Webb Space Telescope (JWST). In Cryogenic Optical Systems and Instruments XII. Edited by Heaney, James B.; Burriesci, Lawrence G.. Proceedings of the SPIE, Volume 6692, pp. 66920M (2007)., volume 6692 of Presented at the Society of Photo-Optical Instrumentation Engineers (SPIE) Conference, October 2007.

[11] M. Griffin, A. Abergel, P. Ade, P. André, J.-P. Baluteau, J. Bock, A. Franceschini, W. Gear, J. Glenn, D. Griffin, K. King, E. Lellouch, D. Naylor, G. Olofsson, I. Perez-Fournon, M. Rowan-Robinson, P. Saraceno, E. Sawyer, A. Smith, B. Swinyard, L. Vigroux, and G. Wright. Herschel-SPIRE: design, performance, and scientific capabilities. In Space Telescopes and Instrumentation I: Optical, Infrared, and Millimeter. Edited by Mather, John C.; MacEwen, Howard A.; de Graauw, Mattheus W. M.. Proceedings of the SPIE, Volume 6265, pp. 62650A (2006)., volume 6265 of Presented at the Society of PhotoOptical Instrumentation Engineers (SPIE) Conference, July 2006.

[12] P. J. Love, A. W. Hoffman, N. A. Lum, K. J. Ando, J. Rosbeck, W. D. Ritchie, N. J. Therrien, R. S. Holcombe, and E. Corrales. 1024 x 1024 Si:As IBC detector arrays for JWST MIRI. In T. J. Grycewicz and C. J. Marshall, editors, Focal Plane Arrays for Space Telescopes II. Edited by Grycewicz, Thomas J.; Marshall, Cheryl J. Proceedings of the SPIE, Volume 5902, pp. 58-66 (2005)., volume 5902 of Presented at the Society of Photo-Optical Instrumentation Engineers (SPIE) Conference, August 2005.

[13] T. Onaka and T. Nakagawa. SPICA: A $3.5 \mathrm{~m}$ space infrared telescope for mid- and far-infrared astronomy. Advances in Space Research, 36:1123-1127, 2005.

[14] Bruce Swinyard. Esi: the far-infrared instrument for the spica mission. volume 6265, page 62650L. SPIE, 2006.

[15] F. Helmich and R. Ivison. FIRI - a Far-Infrared Interferometer. ArXiv eprints, 707, July 2007. 
[16] D. Leisawitz, C. Baker, A. Barger, D. Benford, A. Blain, R. Boyle, R. Broderick, J. Budinoff, J. Carpenter, R. Caverly, P. Chen, S. Cooley, C. Cottingham, J. Crooke, D. Dipietro, M. Dipirro, M. Femiano, A. Ferrer, J. Fischer, J. P. Gardner, L. Hallock, K. Harris, K. Hartman, M. Harwit, L. Hillenbrand, T. Hyde, D. Jones, J. Kellogg, A. Kogut, M. Kuchner, B. Lawson, J. Lecha, M. Lecha, A. Mainzer, J. Mannion, A. Martino, P. Mason, J. Mather, G. McDonald, R. Mills, L. Mundy, S. Ollendorf, J. Pellicciotti, D. Quinn, K. Rhee, S. Rinehart, T. Sauerwine, R. Silverberg, T. Smith, G. Stacey, H. P. Stahl, J. Staguhn, S. Tompkins, J. Tveekrem, S. Wall, and M. Wilson. The space infrared interferometric telescope (SPIRIT): High-resolution imaging and spectroscopy in the far-infrared. Advances in Space Research, 40:689-703, 2007.

[17] A. L. Woodcraft, P. A. R. Ade, D. Bintley, J. S. House, C. L. Hunt, R. V. Sudiwala, W. B. Doriese, W. D. Duncan, G. C. Hilton, K. D. Irwin, C. D. Reintsema, J. N. Ullom, M. D. Audley, M. A. Ellis, W. S. Holland, M. Macintosh, C. C. Dunare, W. Parkes, A. J. Walton, J. B. Kycia, M. Halpern, and E. Schulte. Electrical and optical measurements on the first SCUBA-2 prototype 1280 pixel submillimeter superconducting bolometer array. Review of Scientific Instruments, 78:4502-+, February 2007.

[18] M. D. Niemack, Y. Zhao, E. Wollack, R. Thornton, E. R. Switzer, D. S. Swetz, S. T. Staggs, L. Page, O. Stryzak, H. Moseley, T. A. Marriage, M. Limon, J. M. Lau, J. Klein, M. Kaul, N. Jarosik, K. D. Irwin, A. D. Hincks, G. C. Hilton, M. Halpern, J. W. Fowler, R. P. Fisher, R. Dünner, W. B. Doriese, S. R. Dicker, M. J. Devlin, J. Chervenak, B. Burger, E. S. Battistelli, J. Appel, M. Amiri, C. Allen, and A. M. Aboobaker. A Kilopixel Array of TES Bolometers for ACT: Development, Testing, and First Light. Journal of Low Temperature Physics, pages 114-+, January 2008.

[19] N. Billot, P. Agnèse, J.-L. Auguères, A. Béguin, A. Bouère, O. Boulade, C. Cara, C. Cloué, E. Doumayrou, L. Duband, B. Horeau, I. le Mer, J. Lepennec, J. Martignac, K. Okumura, V. Revéret, M. Sauvage, F. Simoens, and L. Vigroux. The Herschel/PACS 2560 bolometers imaging camera. In Space Telescopes and Instrumentation I: Optical, Infrared, and Millimeter. Edited by Mather, John C.; MacEwen, Howard A.; de Graauw, Mattheus W. M.. Proceedings of the SPIE, Volume 6265, pp. 62650D (2006)., volume 6265 of Presented at the Society of Photo-Optical Instrumentation Engineers (SPIE) Conference, July 2006.

[20] S. E. Church, M. C. Price, M. J. Griffin, P. A. R. Ade, R. J. Emery, and B. M. Swinyard. Non-linear effects in doped-germanium photoconductors for the ISO Long Wavelength Spectrometer. In T. D. Guyenne and J. Hunt, editors, Photon Detectors for Space Instrumentation, volume 356 of ESA Special Publication, pages 261-264, December 1992. 
[21] M. Kenyon, P. K. Day, C. M. Bradford, J. J. Bock, and H. G. Leduc. Progress on background-limited membrane-isolated TES bolometers for farIR/submillimeter spectroscopy. In Millimeter and Submillimeter Detectors and Instrumentation for Astronomy III. Edited by Zmuidzinas, Jonas; Holland, Wayne S.; Withington, Stafford; Duncan, William D.. Proceedings of the SPIE, Volume 6275, pp. 627508 (2006)., volume 6275 of Presented at the Society of Photo-Optical Instrumentation Engineers (SPIE) Conference, July 2006 .

[22] P. A. J. de Korte, J. V. Anquita, X. Barcons, P. Bastia, J. Beyer, F. Briones, C. Brockley Blatt, M. Bruijn, J. Bussons, A. Camon, M. T. Ceballos, D. Drung, C. Enns, L. Fabrega, G. Fraser, F. Gatti, L. Gottardi, W. Hajdas, P. Helistö, I. Hepburn, J.-W. den Herder, H. Hoevers, Y. Ishisaki, M. Kiviranta, J. van der Kuur, A. Mchedlishvili, K. Mitsuda, L. Piro, J. Sese, N. Yamasaki, and C. Whitford. EURECA: a European-Japanese micro-calorimeter array. In Space Telescopes and Instrumentation II: Ultraviolet to Gamma Ray. Edited by Turner, Martin J. L.; Hasinger, Günther. Proceedings of the SPIE, Volume 6266, pp. 62661Z (2006)., volume 6266 of Presented at the Society of Photo-Optical Instrumentation Engineers (SPIE) Conference, July 2006.

[23] K. D. Irwin, M. D. Audley, J. A. Beall, J. Beyer, S. Deiker, W. Doriese, W. Duncan, G. C. Hilton, W. Holland, C. D. Reintsema, J. N. Ullom, L. R. Vale, and Y. Xu. In-focal-plane SQUID multiplexer. Nuclear Instruments and Methods in Physics Research A, 520:544-547, March 2004.

[24] G. H. Rieke. Detection of light from the Ultraviolet to the Submillimeter. Cambridge University Press, 1994.

[25] A. Poglitsch, C. Waelkens, O. H. Bauer, J. Cepa, H. Feuchtgruber, T. Henning, C. van Hoof, F. Kerschbaum, D. Lemke, E. Renotte, L. Rodriguez, P. Saraceno, and B. Vandenbussche. The photodetector array camera and spectrometer (PACS) for the Herschel Space Observatory. In Space Telescopes and Instrumentation I: Optical, Infrared, and Millimeter. Edited by Mather, John C.; MacEwen, Howard A.; de Graauw, Mattheus W. M.. Proceedings of the SPIE, Volume 6265, pp. 62650B (2006)., volume 6265 of Presented at the Society of Photo-Optical Instrumentation Engineers (SPIE) Conference, July 2006.

[26] N. W. Ashcroft and N. D. Mermin. Solid State Physics. Saunders College Publishing, 1976.

[27] F. London and H. London. The Electromagnetic Equations of the Superconductor. Royal Society of London Proceedings Series A, 149:71-88, March 1935.

[28] C. J. Gorter and H. Casimir. On superconductivity I. Physica, 1:306-320, 1934. 
[29] J. Bardeen, L. N. Cooper, and J. R. Schrieffer. Theory of Superconductivity. Physical Review, 108:1175-1204, December 1957.

[30] A. C. Rose-Innes and E. H. Rhoderick. Introduction to Superconductivity. Robert Maxwell (publisher at Pergamon press), 1980.

[31] T. Van Duzer and C. W. Turner. Principles of Superconducting Devices and Circuits. Edward Arnold, 1981.

[32] M Tinkham. Introduction to Superconductivity. McGraw-Hill Inc, 1975.

[33] G Vardulakis. Superconducting Kinetic Inductance Detectors, Theory, Simulations and Experiments. PhD thesis, Cambridge University, Astrophysics Group - UK, May 2007.

[34] A. B. Pippard. An Experimental and Theoretical Study of the Relation between Magnetic Field and Current in a Superconductor. Royal Society of London Proceedings Series A, 216:547-568, February 1953.

[35] D. C. Mattis and J. Bardeen. Theory of the Anomalous Skin Effect in Normal and Superconducting Metals. Physical Review, 111:412-417, July 1958.

[36] B. Geilikman and V. Kresin. Kinetic and Nonsteady-state Effects in Superconductors. John Wiley and sons, 1974.

[37] W. Eisenmenger. Nonequilibrium Superconductivity, Phonons and Kapitza Boundaries Chapter 3. New York: Plenum Press, 1981.

[38] S. B. Kaplan, C. C. Chi, D. N. Langenberg, J. J. Chang, S. Jafarey, and D. J. Scalapino. Quasiparticle and phonon lifetimes in superconductors. Phys. Rev. B, 14:4854-4873, December 1976.

[39] I. S. Grant and W. R. Phillips. Electro-magnitism. Wiley, 1990.

[40] D. M. Pozar. Microwave Engineering. Wiley, 2005.

[41] A. Porch, P. Mauskopf, S. Doyle, and C. Dunscombe. Calculation of the characteristics of coplanar resonators for kinetic inductance detectors. Applied Superconductivity, IEEE Transactions on, 15(2):552-555, June 2005.

[42] L. Li, L. Frunzio, C. M. Wilson, and D. E. Prober. Quasiparticle nonequilibrium dynamics in a superconducting Ta film. Journal of Applied Physics, 93:1137-1141, January 2003.

[43] Sonnet Software Inc., 2004. Sonnet user manual, Release 10.

[44] Prof Peter Ade and Dr Carole Tucker. Private Communication. 
[45] A. D. O'Connell, M. Ansmann, R. C. Bialczak, M. Hofheinz, N. Katz, E. Lucero, C. McKenney, M. Neeley, H. Wang, E. M. Weig, A. N. Cleland, and J. M. Martinis. Microwave Dielectric Loss at Single Photon Energies and milliKelvin Temperatures. ArXiv e-prints, 802, February 2008.

[46] A. Vayonakis, C. Luo, H. G. Leduc, R. Schoelkopf, and J. Zmuidzinas. The millimeter-wave properties of superconducting microstrip lines. Low Temperature Detectors, 605:539-542, February 2002.

[47] J. G. Hartnett, M. E. Tobar, and E. N. Ivanov. High Resolution RoomTemperature Determination of the Loss Tangent of Sapphire using the Whispering-Gallery-Mode Method. In 2001 Asia-Pacific Radio Science Conference $A P-R A S C$ '01, pages 283-+, 2001.

[48] Kyocera. Single crystal sapphire, http://americas.kyocera.com/kicc/pdf/kyocera\%20sapphire.pdf, Unknown.

[49] A. B. Pippard. The Physics of Vibration, Vol. 1. Cambridge University Press, 1978.

[50] J. Baselmans, S. J. C. Yates, R. Barends, Y. J. Y. Lankwarden, J. R. Gao, H. Hoevers, and T. M. Klapwijk. Noise and Sensitivity of Aluminum Kinetic Inductance Detectors for Sub-mm Astronomy. Journal of Low Temperature Physics, 151:524-529, April 2008.

[51] Dr Jochem Baselmans and Dr Stephen Yates. Private Communication.

[52] F. F. Enectalí. THEORY AND DEVELOPMENT OF POSITIONSENSITIVE QUANTUM CALORIMETERS. PhD thesis, Stanford University, United States - California, 2001.

[53] D. R. Lied and H. P. R Frederikse. CRC Handbook of Chemistry and Physics . CRC Press, 1997.

[54] P. D. Mauskopf, J. J. Bock, H. del Castillo, W. L. Holzapfel, and A. E. Lange. Composite infrared bolometers with Si 3 N 4 micromesh absorbers. Appl. Opt., 36:765-771, February 1997.

[55] Dr Giorgio Savini. Private Communication.

[56] UK Astronomy Technology Centre. Scuba-ii web page, http://www.roe.ac.uk/ukatc/projects/scubatwo/whatis/summary.html, 2002.

[57] E. Hecht. Optics. Addison-Wesley, 1998.

[58] Rogers Corporation. High frequency laminates data sheet, http://www.rogerscorporation.com/acm/litintbl.htm, Unknown. 
[59] R. Ulrich. Far-infrared properties of metallic mesh and its complementary structure. Infrared Physics, 7:37-50, March 1967.

[60] G. Saklatvala, S. Withington, and M. P. Hobson. Coupled-mode theory for infrared and submillimeter wave detectors. Journal of the Optical Society of America A, 24:764-775, March 2007.

[61] Chris Dunscombe. Private Communication.

[62] P. Verhoeve, N. Rando, J. Verveer, A. Peacock, A. van Dordrecht, P. Videler, M. Bavdaz, D. J. Goldie, T. Lederer, F. Scholze, G. Ulm, and R. Venn. Response of niobium-based superconducting tunnel junctions in the soft-x-ray region 0.15-6.5 keV. Phys. Rev. B, 53:809-817, January 1996.

[63] J. Haisma, G. A. C. M. Spierings, U. K. P. Biermann, and J. A. Pals. Silicon-on-Insulator Wafer Bonding-Wafer Thinning Technological Evaluations. Japanese Journal of Applied Physics, 28:1426-+, August 1989.

[64] EPO-TEK. Silver epoxy data sheet, http://www.epotek.com/sscdocs/datasheets/h20e.pdf, Unknown.

[65] Janice. Janice, http://www.janis.com/index.htm, Unknown.

[66] Dr S.T. Chase. Chase cryogenics, http://www.chasecryogenics.com/about.htm, Unknown.

[67] Coax.co. Low temperature microwave cable data sheet, http://www.coax.co.jp/english/semi/086.html, Unknown.

[68] A. Kushino, M. Ohkubo, and K. Fujioka. Thermal conduction measurement of miniature coaxial cables between 0.3 and $4.5 \mathrm{~K}$ for the wiring of superconducting detectors. Cryogenics, 45:637-640, September 2005.

[69] Dr Ian Rycroft. Qmc instrument's home page, http://www.terahertz.co.uk/index.html, 2007.

[70] Lakshore. Grt technical data, http://www.lakeshore.com/temp/sen/gtrd.html, 2007.

[71] Aeroflex. 8310 data sheet, http://www.weinschel.com/pdfiles/wmod8310.pdf, 2007.

[72] Dr. Ir. Jochem Baselmans. Sron kinetic inductance research web page, http://www.sron.nl/index.php?option=com_content\&task=view\&id=46\&itemid= Unknown. 
[73] P. Hargrave, T. Waskett, T. Lim, and B. Swinyard. Performance of flightmodel on-board calibration sources on Herschel-SPIRE. In Millimeter and Submillimeter Detectors and Instrumentation for Astronomy III. Edited by Zmuidzinas, Jonas; Holland, Wayne S.; Withington, Stafford; Duncan, William D.. Proceedings of the SPIE, Volume 6275, pp. 627514 (2006)., volume 6275 of Presented at the Society of Photo-Optical Instrumentation Engineers (SPIE) Conference, July 2006.

[74] P. A. R. Ade, G. Pisano, C. Tucker, and S. Weaver. A review of metal mesh filters. In Millimeter and Submillimeter Detectors and Instrumentation for Astronomy III. Edited by Zmuidzinas, Jonas; Holland, Wayne S.; Withington, Stafford; Duncan, William D.. Proceedings of the SPIE, Volume 6275, pp. 62750U (2006)., volume 6275 of Presented at the Society of Photo-Optical Instrumentation Engineers (SPIE) Conference, July 2006.

[75] H. D. Young and R. A. Freedman. University Physics. Addison-Wesley, 1996.

[76] ITT Visual information solutions, 2004. IDL Reference Guide volumes 1 and 2.

[77] J. Gao, J. Zmuidzinas, B. A. Mazin, H. G. Leduc, and P. K. Day. Noise properties of superconducting coplanar waveguide microwave resonators. Applied Physics Letters, 90:2507-+, March 2007.

[78] Miteq. 1to $18 \mathrm{ghz}$ image rejection or i/q mixers, http://amps.miteq.com/datasheets/miteq-irm0118.pdf, Unknown.

[79] B. A. Mazin, M. E. Eckart, B. Bumble, S. Golwala, P. K. Day, J. Gao, and J. Zmuidzinas. Optical/UV and X-Ray Microwave Kinetic Inductance Strip Detectors. Journal of Low Temperature Physics, 151:537-543, April 2008.

[80] A. G. Kozorezov, A. F. Volkov, J. K. Wigmore, A. Peacock, A. Poelaert, and R. den Hartog. Quasiparticle-phonon downconversion in nonequilibrium superconductors. Phys. Rev. B, 61:11807-11819, May 2000.

[81] Porch. Kinetic and magnetic inductance in thin films, unpublished. 